\title{
Sending a Bi-Variate Gaussian over a Gaussian MAC
}

\author{
Amos Lapidoth Stephan Tinguely
}

\begin{abstract}
We study the power versus distortion trade-off for the distributed transmission of a memoryless bi-variate Gaussian source over a two-to-one average-power limited Gaussian multiple-access channel. In this problem, each of two separate transmitters observes a different component of a memoryless bi-variate Gaussian source. The two transmitters then describe their source component to a common receiver via an average-power constrained Gaussian multiple-access channel. From the output of the multiple-access channel, the receiver wishes to reconstruct each source component with the least possible expected squared-error distortion. Our interest is in characterizing the distortion pairs that are simultaneously achievable on the two source components.

We present sufficient conditions and necessary conditions for the achievability of a distortion pair. These conditions are expressed as a function of the channel signal-to-noise ratio (SNR) and of the source correlation. In several cases the necessary conditions and sufficient conditions are shown to agree. In particular, we show that if the channel SNR is below a certain threshold, then an uncoded transmission scheme is optimal. We also derive the precise high-SNR asymptotics of an optimal scheme.

】
\end{abstract}

\section{Introduction}

We study the power versus distortion trade-off for the distributed transmission of a memoryless bi-variate Gaussian source over a two-to-one average-power limited Gaussian multiple-access channel. In this problem, each of two separate transmitters observes a different component of a memoryless bi-variate Gaussian source. The two transmitters then describe their source component to a common receiver via an averagepower constrained Gaussian multiple-access channel. From the output of the multipleaccess channel, the receiver wishes to reconstruct each source component with the least possible expected squared-error distortion. Our interest is in characterizing the distortion pairs that are simultaneously achievable on the two source components.

We present sufficient conditions and necessary conditions for the achievability of a distortion pair. These conditions are expressed as a function of the channel signal-tonoise ratio (SNR) and of the source correlation. In several cases the necessary conditions

The work of Stephan Tinguely was partially supported by the Swiss National Science Foundation under Grant 200021-111863/1. The results in this paper were presented in part at the 2006 IEEE Communications Theory Workshop, Dorado, Puerto Rico and at the 2006 IEEE International Symposium on Information Theory, Seattle, USA.

A. Lapidoth and S. Tinguely are with the Signal and Information Processing Laboratory (ISI), ETH Zurich, Switzerland (e-mail: lapidoth@isi.ee.ethz.ch; tinguely@isi.ee.ethz.ch). 
and sufficient conditions are shown to agree, thus yielding a full characterization of the achievable distortions. In particular, we show that if the channel SNR is below a certain threshold (that we compute), then an uncoded transmission scheme is optimal. We also derive the precise high-SNR asymptotics of an optimal scheme. The uncoded result is reminiscent of Goblick's result [1] that for the transmission of a Gaussian source over an AWGN channel the minimal squared-error distortion is achieved by uncoded transmission. But in our setting uncoded transmission is only optimal for some SNRs.

Our problem can be viewed as a lossy Gaussian version of the problem addressed by Cover, El Gamal and Salehi [2] (see also [3, 4]) in which a bi-variate finite-alphabet source is to be transmitted losslessly over a two-to-one multiple-access channel. Our problem is also related to the quadratic Gaussian two-terminal source-coding problem [5, 6] and to the quadratic Gaussian CEO problem [7, 8]. In both of these problems, correlated Gaussians are described distributedly to a central receiver. But, in the quadratic Gaussian CEO problem the interest is in reconstructing a single Gaussian random variable that underlies the observations of the different transmitters, rather than reconstructing each transmitter's observation itself. But more importantly, the above two problems are source-coding problems whereas ours is one of combined sourcechannel coding. We emphasize that, as our results show, source-channel separation is suboptimal for our setting.

The problem of transmitting correlated sources over multiple-access channels has so far only been studied sparsely. One of the first results is due to Cover, El Gamal and Salehi [2] who presented sufficient conditions for the lossless transmission of a finitealphabet bi-variate source over a multiple-access channel. Later, several variations of this problem were considered. Salehi [9] studied a lossy versions of the problem with a finite-alphabet source and arbitrary distortion measures on each source component. For this problem he derived sufficient conditions for the achievability of a distortion pair. More recently, another variation where the two source components are binary with Hamming distortion and where the multiple-access channel is Gaussian was considered by Murugan, Gopala and El Gamal [10] who derived sufficient conditions for the achievability of a distortion pair. Gastpar [11] considered a combined source-channel coding analog of the quadratic Gaussian CEO problem. In this problem, distributed transmitters observe independently corrupted versions of the same univariate Gaussian source. These transmitters are connected to a central receiver by means of a many-toone Gaussian multiple-access channel. The central receiver wishes to reconstruct the original univariate source as accurately as possible. For this problem, Gastpar showed that the minimal expected squared-error distortion is achieved by an uncoded transmission scheme. The extension of our problem to the case where perfect causal feedback from the receiver to each transmitter is available is studied in [12] (see also [13]).

\section{Problem Statement}

\section{$2.1 \quad$ Setup}

Our setup is illustrated in Figure 1, A memoryless bi-variate Gaussian source is connected to a two-to-one Gaussian multiple-access channel. Each transmitter observes one of the source components and wishes to describe it to the common receiver. The 


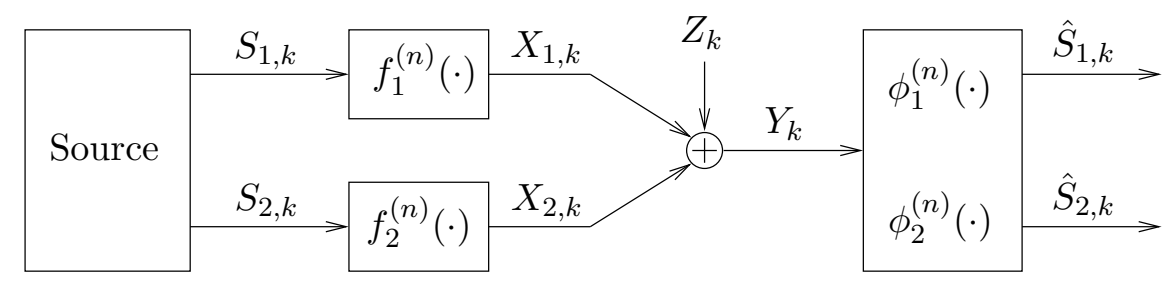

Figure 1: Bi-variate Gaussian source with one-to-two Gaussian multiple-access channel.

source symbols produced at time $k \in \mathbb{Z}$ are denoted by $\left(S_{1, k}, S_{2, k}\right)$. The source output pairs $\left\{\left(S_{1, k}, S_{2, k}\right)\right\}$ are independent identically distributed (IID) zero-mean Gaussians of covariance matrix

$$
\mathrm{K}_{S S}=\left(\begin{array}{cc}
\sigma_{1}^{2} & \rho \sigma_{1} \sigma_{2} \\
\rho \sigma_{1} \sigma_{2} & \sigma_{2}^{2}
\end{array}\right),
$$

where $\rho \in[-1,1]$ and where $0<\sigma_{i}^{2}<\infty, i \in\{1,2\}$. The sequence $\left\{S_{1, k}\right\}$ of the first source component is observed by Transmitter 1 and the sequence $\left\{S_{2, k}\right\}$ of the second source component is observed by Transmitter 2 . The two source components are to be described over the multiple-access channel to the common receiver by means of the channel input sequences $\left\{X_{1, k}\right\}$ and $\left\{X_{2, k}\right\}$, where $x_{1, k} \in \mathbb{R}$ and $x_{2, k} \in \mathbb{R}$. The corresponding time- $k$ channel output is given by

$$
Y_{k}=X_{1, k}+X_{2, k}+Z_{k}
$$

where $Z_{k}$ is the time- $k$ additive noise term, and where $\left\{Z_{k}\right\}$ are IID zero-mean variance$N$ Gaussian random variables that are independent of the source sequence.

For the transmission of the source $\left\{S_{1, k}, S_{2, k}\right\}$, we consider block encoding schemes and denote the block-length by $n$ and the corresponding $n$-sequences in boldface, e.g. $\mathbf{S}_{1}=\left(S_{1,1}, S_{1,2}, \ldots, S_{1, n}\right)$. Transmitter $i$ is modeled as a function $f_{i}^{(n)}: \mathbb{R}^{n} \rightarrow \mathbb{R}^{n}$ which produces the channel input sequence $\mathbf{X}_{i}$ based on the observed source sequence $\mathbf{S}_{i}=\left(S_{i, 1}, S_{i, 2}, \ldots, S_{i, n}\right)$, i.e.

$$
\mathbf{X}_{i}=f_{i}^{(n)}\left(\mathbf{S}_{i}\right) \quad i \in\{1,2\} .
$$

The channel input sequences are subjected to expected average power constraints

$$
\frac{1}{n} \sum_{k=1}^{n} \mathrm{E}\left[X_{i, k}^{2}\right] \leq P_{i} \quad i \in\{1,2\},
$$

for some given $P_{i}>0$.

The decoder consists of two functions $\phi_{i}^{(n)}: \mathbb{R}^{n} \rightarrow \mathbb{R}^{n}, i \in\{1,2\}$, which perform estimates $\hat{\mathbf{S}}_{i}$ of the respective source sequences $\mathbf{S}_{i}$, based on the observed channel output sequence $\mathbf{Y}$, i.e.

$$
\hat{\mathbf{S}}_{i}=\phi_{i}^{(n)}(\mathbf{Y}) \quad i \in\{1,2\} .
$$

Our interest is in the pairs of expected squared-error distortions that can be achieved simultaneously on the source-pair as the blocklength $n$ tends to infinity. In view of this, we next define the notion of achievability. 


\subsection{Achievability of Distortion Pairs}

Definition 2.1. Given $\sigma_{1}, \sigma_{2}>0, \rho \in[-1,1], P_{1}, P_{2}>0$, and $N>0$ we say that the tuple $\left(D_{1}, D_{2}, \sigma_{1}^{2}, \sigma_{2}^{2}, \rho, P_{1}, P_{2}, N\right)$ is achievable if there exists a sequence of encoding functions $\left\{f_{1}^{(n)}, f_{2}^{(n)}\right\}$ as in (3), satisfying the average power constraints (4), and a sequence of reconstruction pairs $\left\{\phi_{1}^{(n)}, \phi_{2}^{(n)}\right\}$ as in (5), such that the average distortions resulting from these encoding and reconstruction functions fulfill

$$
\varlimsup_{n \rightarrow \infty} \frac{1}{n} \sum_{k=1}^{n} \mathrm{E}\left[\left(S_{i, k}-\hat{S}_{i, k}\right)^{2}\right] \leq D_{i}, \quad i=1,2,
$$

whenever

$$
\mathbf{Y}=f_{1}^{(n)}\left(\mathbf{S}_{1}\right)+f_{2}^{(n)}\left(\mathbf{S}_{2}\right)+\mathbf{Z},
$$

and where $\left\{\left(S_{1, k}, S_{2, k}\right)\right\}$ are IID zero-mean bi-variate Gaussian vectors of covariance matrix $\mathrm{K}_{S S}$ as in (11) and $\left\{Z_{k}\right\}$ are IID zero-mean variance- $N$ Gaussians that are independent of $\left\{\left(S_{1, k}, S_{2, k}\right)\right\}$.

The problem we address here is, for given $\sigma_{1}^{2}, \sigma_{2}^{2}, \rho, P_{1}, P_{2}$, and $N$, to find the set of pairs $\left(D_{1}, D_{2}\right)$ such that $\left(D_{1}, D_{2}, \sigma_{1}^{2}, \sigma_{2}^{2}, \rho, P_{1}, P_{2}, N\right)$ is achievable. Sometimes, we will refer to the set of all $\left(D_{1}, D_{2}\right)$ such that $\left(D_{1}, D_{2}, \sigma_{1}^{2}, \sigma_{2}^{2}, \rho, P_{1}, P_{2}, N\right)$ is achievable as the distortion region associated to $\left(\sigma_{1}^{2}, \sigma_{2}^{2}, \rho, P_{1}, P_{2}, N\right)$. In that sense, we will often say, with respect to some $\left(\sigma_{1}, \sigma_{2}, \rho, P_{1}, P_{2}, N\right)$, that the pair $\left(D_{1}, D_{2}\right)$ is achievable, instead of saying that the tuple $\left(D_{1}, D_{2}, \sigma_{1}^{2}, \sigma_{2}^{2}, \rho, P_{1}, P_{2}, N\right)$ is achievable.

\subsection{Normalization}

For the described problem we now show that, without loss in generality, the source law given in (11) can be restricted to a simpler form. This restriction will ease the statement of our results as well as their derivations.

Reduction 2.1. For the problem stated in Sections 2.1 and 2.2 , there is no loss in generality in restricting the source law to satisfy

$$
\sigma_{1}^{2}=\sigma_{2}^{2}=\sigma^{2} \quad \text { and } \quad \rho \in[0,1] .
$$

Proof. The proof follows by noting that the described problem has certain symmetry properties with respect to the source law. We prove the reductions on the source variance and on the correlation coefficient separately.

i) The reduction to correlation coefficients $\rho \in[0,1]$ holds because the optimal distortion region depends on the correlation coefficient only via its absolute value $|\rho|$. That is, the tuple $\left(D_{1}, D_{2}, \sigma_{1}^{2}, \sigma_{2}^{2}, \rho, P_{1}, P_{2}, N\right)$ is achievable if, and only if, the tuple $\left(D_{1}, D_{2}, \sigma_{1}^{2}, \sigma_{2}^{2},-\rho, P_{1}, P_{2}, N\right)$ is achievable. To see this, note that if $\left\{f_{1}^{(n)}, f_{2}^{(n)}, \phi_{1}^{(n)}, \phi_{2}^{(n)}\right\}$ achieves the distortion $\left(D_{1}, D_{2}\right)$ for the source of correlation coefficient $\rho$, then $\left\{\tilde{f}_{1}^{(n)}, f_{2}^{(n)}, \tilde{\phi}_{1}^{(n)}, \phi_{2}^{(n)}\right\}$, where

$$
\tilde{f}_{1}^{(n)}\left(\mathbf{S}_{1}\right)=f_{1}^{(n)}\left(-\mathbf{S}_{1}\right) \quad \text { and } \quad \tilde{\phi}_{1}^{(n)}(\mathbf{Y})=-\phi_{1}^{(n)}(\mathbf{Y})
$$

achieves $\left(D_{1}, D_{2}\right)$ on the source with correlation coefficient $-\rho$. 
ii) The restriction to source variances satisfying $\sigma_{1}^{2}=\sigma_{2}^{2}=\sigma^{2}$ incurs no loss of generality because the distortion region scales linearly with the source variances. That is, the tuple $\left(D_{1}, D_{2}, \sigma_{1}^{2}, \sigma_{2}^{2}, \rho, P_{1}, P_{2}, N\right)$ is achievable if, and only if, for every $\alpha_{1}, \alpha_{2} \in \mathbb{R}^{+}$, the tuple $\left(\alpha_{1} D_{1}, \alpha_{2} D_{2}, \alpha_{1} \sigma_{1}^{2}, \alpha_{2} \sigma_{2}^{2}, \rho, P_{1}, P_{2}, N\right)$ is achievable. This can be seen as follows. If $\left\{f_{1}^{(n)}, f_{2}^{(n)}, \phi_{1}^{(n)}, \phi_{2}^{(n)}\right\}$ achieves $\left(D_{1}, D_{2}, \sigma_{1}^{2}, \sigma_{2}^{2}, \rho\right.$, $\left.P_{1}, P_{2}, N\right)$, then the combination of the encoders

$$
\tilde{f}_{i}^{(n)}\left(\mathbf{S}_{i}\right)=f_{i}^{(n)}\left(\mathbf{S}_{i} / \sqrt{\alpha_{i}}\right), \quad i \in\{1,2\}
$$

with the reconstructors

$$
\tilde{\phi}_{i}^{(n)}(\mathbf{Y})=\sqrt{\alpha_{i}} \cdot \phi_{i}^{(n)}(\mathbf{Y}), \quad i \in\{1,2\}
$$

achieves the tuple $\left(\alpha_{1} D_{1}, \alpha_{2} D_{2}, \alpha_{1} \sigma_{1}^{2}, \alpha_{2} \sigma_{2}^{2}, \rho, P_{1}, P_{2}, N\right)$. And by an analogous argument it follows that if $\left(\alpha_{1} D_{1}, \alpha_{2} D_{2}, \alpha_{1} \sigma_{1}^{2}, \alpha_{2} \sigma_{2}^{2}, \rho, P_{1}, P_{2}, N\right)$ is achievable, then also $\left(D_{1}, D_{2}, \sigma_{1}^{2}, \sigma_{2}^{2}, \rho, P_{1}, P_{2}, N\right)$ is achievable.

In view of Reduction 2.1 we assume for the remainder that the source law additionally satisfies (6).

\section{4 "Symmetric Version" and a Convexity Property}

The "symmetric version" of our problem corresponds to the case where the transmitters are subjected to the same power constraint, and where we seek to achieve the same distortion on each source component. That is, $P_{1}=P_{2}=P$, and we are interested in the minimal distortion

$$
D^{*}\left(\sigma^{2}, \rho, P, N\right) \triangleq \inf \left\{D:\left(D, D, \sigma^{2}, \sigma^{2}, \rho, P, P, N\right) \text { is achievable }\right\},
$$

that is simultaneously achievable on $\left\{S_{1, k}\right\}$ and on $\left\{S_{2, k}\right\}$. In this case, we define the SNR as $P / N$ and seek the distortion $D^{*}\left(\sigma^{2}, \rho, P, N\right)$, for some fixed $\sigma^{2}$ and $\rho$, and as a function of the SNR.

We conclude this section with a convexity property of the achievable distortions.

Remark 2.1. If $\left(D_{1}, D_{2}, \sigma_{1}^{2}, \sigma_{2}^{2}, \rho, P_{1}, P_{2}, N\right)$ and $\left(\tilde{D}_{1}, \tilde{D}_{2}, \sigma_{1}^{2}, \sigma_{2}^{2}, \rho, \tilde{P}_{1}, \tilde{P}_{2}, N\right)$ are achievable, then

$$
\left(\lambda D_{1}+\bar{\lambda} \tilde{D}_{1}, \lambda D_{2}+\bar{\lambda} \tilde{D}_{2}, \sigma_{1}^{2}, \sigma_{2}^{2}, \rho, \lambda P_{1}+\bar{\lambda} \tilde{P}_{1}, \lambda P_{2}+\bar{\lambda} \tilde{P}_{2}, N\right)
$$

is also achievable for every $\lambda \in[0,1]$, where $\bar{\lambda}=(1-\lambda)$.

Proof. Follows by a time-sharing argument.

\section{Preliminaries: Sending a Bi-Variate Gaussian over an AWGN Channel}

In this section we lay the ground for our main results. We study a point-to-point analog of the multiple-access problem described in Section 2.1. More concretely, we 
consider the transmission of a memoryless bi-variate Gaussian source, subject to expected squared-error distortion on each source component, over the additive white Gaussian noise (AWGN) channel. For this problem, we characterize the power versus distortion trade-off and show that below a certain SNR threshold, an uncoded transmission scheme is optimal. This problem is simpler than our multiple-access problem because here source-channel separation is optimal.

\subsection{Problem Statement}

The setup considered in this section is illustrated in Figure 2, The difference to the

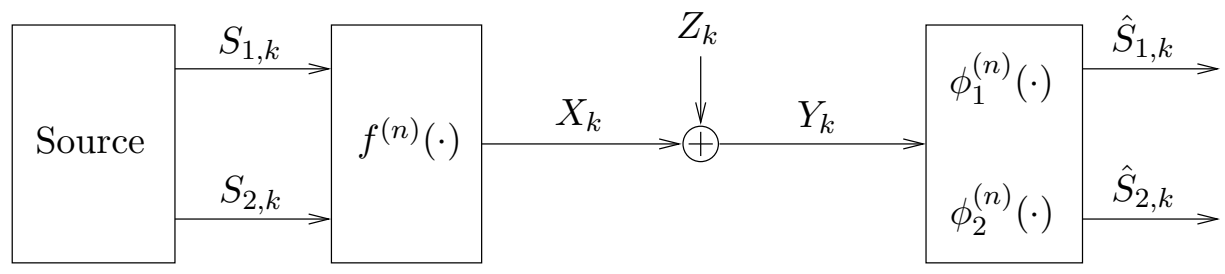

Figure 2: Bi-variate Gaussian source with additive white Gaussian noise channel.

multiple-access problem of Section 2.1 is that now the two source sequences $\mathbf{S}_{1}$ and $\mathbf{S}_{2}$ are observed and transmitted jointly by one single transmitter rather than by two distributed transmitters. Thus, the channel input sequence $\mathbf{X}$ is a function $f^{(n)}: \mathbb{R}^{n} \times$ $\mathbb{R}^{n} \rightarrow \mathbb{R}^{n}$ of the source sequences $\left(\mathbf{S}_{1}, \mathbf{S}_{2}\right)$, i.e.

$$
\mathbf{X}=f^{(n)}\left(\mathbf{S}_{1}, \mathbf{S}_{2}\right) .
$$

This channel input sequence is subjected to an average power constraint

$$
\frac{1}{n} \sum_{k=1}^{n} \mathrm{E}\left[X_{k}^{2}\right] \leq P,
$$

for some given $P>0$.

The remainder of the problem statement is as in the multiple-access problem. The source law is assumed to be given by (11) and to satisfy ([6). The reconstruction functions are as defined in (5), and the achievability of distortion pairs is defined analogously as in Section 2.1. Our interest is in the set of achievable distortion pairs $\left(D_{1}, D_{2}\right)$.

\subsection{Rate-Distortion Function of a Bi-Variate Gaussian}

Denoting the rate-distortion function of the source $\left\{\left(S_{1, k}, S_{2, k}\right)\right\}$ by $R_{S_{1}, S_{2}}\left(D_{1}, D_{2}\right)$, the set of achievable distortion pairs is given by all pairs $\left(D_{1}, D_{2}\right)$ satisfying

$$
R_{S_{1}, S_{2}}\left(D_{1}, D_{2}\right) \leq \frac{1}{2} \log _{2}\left(1+\frac{P}{N}\right) .
$$

We next compute the rate-distortion function $R_{S_{1}, S_{2}}\left(D_{1}, D_{2}\right)$. 
Theorem 3.1. The rate-distortion function $R_{S_{1}, S_{2}}\left(D_{1}, D_{2}\right)$ is given by

$$
R_{S_{1}, S_{2}}\left(D_{1}, D_{2}\right)= \begin{cases}\frac{1}{2} \log _{2}^{+}\left(\frac{\sigma^{2}}{D_{\min }}\right) & \text { if }\left(D_{1}, D_{2}\right) \in \mathscr{D}_{1} \\ \frac{1}{2} \log _{2}^{+}\left(\frac{\sigma^{4}\left(1-\rho^{2}\right)}{D_{1} D_{2}}\right) & \text { if }\left(D_{1}, D_{2}\right) \in \mathscr{D}_{2} \\ \frac{1}{2} \log _{2}^{+}\left(\frac{\sigma^{4}\left(1-\rho^{2}\right)}{D_{1} D_{2}-\left(\rho \sigma^{2}-\sqrt{\left(\sigma^{2}-D_{1}\right)\left(\sigma^{2}-D_{2}\right)}\right)^{2}}\right) & \text { if }\left(D_{1}, D_{2}\right) \in \mathscr{D}_{3} .\end{cases}
$$

where $\log _{2}^{+}(x)=\max \left\{0, \log _{2}(x)\right\}, D_{\min }=\min \left\{D_{1}, D_{2}\right\}$ and where the regions $\mathscr{D}_{1}, \mathscr{D}_{2}$ and $\mathscr{D}_{3}$ are given by

$$
\begin{aligned}
& \mathscr{D}_{1}=\left\{\begin{array}{c}
\left(D_{1}, D_{2}\right): 0 \leq D_{1} \leq \sigma^{2}\left(1-\rho^{2}\right), D_{2} \geq \sigma^{2}\left(1-\rho^{2}\right)+\rho^{2} D_{1} ; \\
\sigma^{2}\left(1-\rho^{2}\right)<D_{1} \leq \sigma^{2}, D_{2} \geq \sigma^{2}\left(1-\rho^{2}\right)+\rho^{2} D_{1}, \\
\mathscr{D}_{2}=\left\{\begin{array}{c}
\left(D_{1}, D_{2}\right): 0 \leq D_{1} \leq \sigma^{2}\left(1-\rho^{2}\right), 0 \leq D_{2}<\left(\sigma^{2}\left(1-\rho^{2}\right)-D_{1}\right) \\
\rho^{2}
\end{array}\right\}, \\
\mathscr{D}_{3}=\left\{\begin{array}{c}
\left(D_{1}-D_{1}\right. \\
\left.D_{2}\right): 0 \leq D_{1} \leq \sigma^{2}\left(1-\rho^{2}\right),
\end{array}\right. \\
\left(\sigma^{2}\left(1-\rho^{2}\right)-D_{1}\right) \frac{\sigma^{2}}{\sigma^{2}-D_{1}} \leq D_{2}<\sigma^{2}\left(1-\rho^{2}\right)+\rho^{2} D_{1} ; \\
\left.\sigma^{2}\left(1-\rho^{2}\right)<D_{1} \leq \sigma^{2}, \frac{D_{1}-\sigma^{2}\left(1-\rho^{2}\right)}{\rho^{2}}<D_{2}<\sigma^{2}\left(1-\rho^{2}\right)+\rho^{2} D_{1}\right\} .
\end{array}\right.
\end{aligned}
$$

Proof. See Appendix A.

The result of Theorem 3.1 was also established independently (and by a different proof) in [14]. The regions $\mathscr{D}_{1}, \mathscr{D}_{2}$, and $\mathscr{D}_{3}$ are illustrated in Figure 3 ,

Remark 3.1. Let $R_{S_{1}}\left(D_{1}\right)$ denote the rate-distortion function for the source component $\left\{S_{1, k}\right\}$, i.e.,

$$
R_{S_{1}}\left(D_{1}\right)=\frac{1}{2} \log _{2}^{+}\left(\frac{\sigma^{2}}{D_{1}}\right)
$$

and let $R_{S_{2} \mid S_{1}}\left(D_{2}\right)$ denote the rate-distortion function for $\left\{S_{2, k}\right\}$ when $\left\{S_{1, k}\right\}$ is given as side-information to both, the encoder and the decoder, i.e.,

$$
R_{S_{2} \mid S_{1}}\left(D_{2}\right)=\frac{1}{2} \log _{2}^{+}\left(\frac{\sigma^{2}\left(1-\rho^{2}\right)}{D_{2}}\right) .
$$




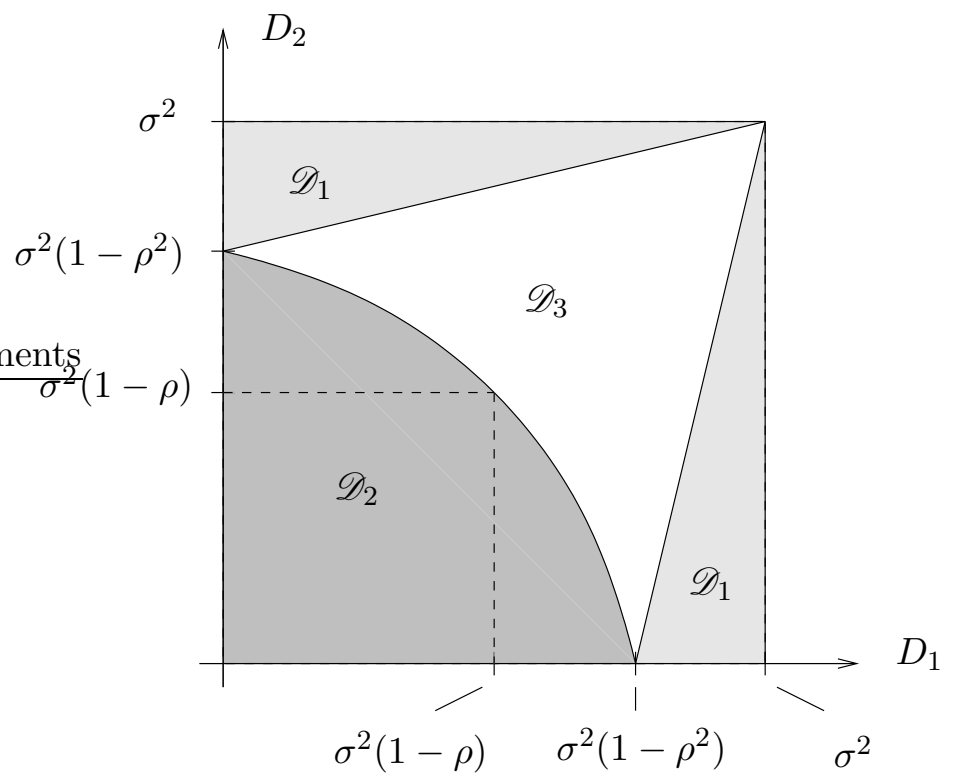

Figure 3: The regions $\mathscr{D}_{1}, \mathscr{D}_{2}, \mathscr{D}_{3}$.

Then, for every $\left(D_{1}, D_{2}\right) \in \mathscr{D}_{2}$ the rate-distortion function $R_{S_{1}, S_{2}}\left(D_{1}, D_{2}\right)$ satisfies

$$
\begin{aligned}
R_{S_{1}, S_{2}}\left(D_{1}, D_{2}\right) & =\frac{1}{2} \log _{2}^{+}\left(\frac{\sigma^{4}\left(1-\rho^{2}\right)}{D_{1} D_{2}}\right) \\
& \stackrel{a)}{=} \frac{1}{2} \log _{2}^{+}\left(\frac{\sigma^{2}}{D_{1}}\right)+\frac{1}{2} \log _{2}^{+}\left(\frac{\sigma^{2}\left(1-\rho^{2}\right)}{D_{2}}\right) \\
& =R_{S_{1}}\left(D_{1}\right)+R_{S_{2} \mid S_{1}}\left(D_{2}\right),
\end{aligned}
$$

where $a$ ) holds since for $\left(D_{1}, D_{2}\right) \in \mathscr{D}_{2}$ we have $D_{1}, D_{2} \geq 0$.

\subsection{Optimal Uncoded Scheme}

As an alternative to the separation-based approach, we now present an uncoded scheme that, for all SNR below a certain threshold, is optimal. The optimality of this uncoded scheme will be useful for understanding a similar result in the multiple-access problem.

The uncoded scheme can be described as follows. At every time instant $k$, the transmitter produces a channel input $X_{k}^{\mathrm{u}}$ of the form

$$
X_{k}^{\mathrm{u}}(\alpha, \beta)=\sqrt{\frac{P}{\sigma^{2}\left(\alpha^{2}+2 \rho \alpha \beta+\beta^{2}\right)}}\left(\alpha S_{1, k}+\beta S_{2, k}\right) \quad k \in\{1,2, \ldots, n\},
$$

for some $\alpha, \beta \in \mathbb{R}$. From the resulting channel output $Y_{k}$, the receiver makes a minimum mean squared-error (MMSE) estimate $\hat{S}_{i, k}^{\mathrm{u}}, i \in\{1,2\}$, of the source sample $S_{i, k}$, i.e.,

$$
\hat{S}_{i, k}^{\mathrm{u}}=\mathrm{E}\left[S_{i, k} \mid Y_{k}\right], \quad i \in\{1,2\} .
$$


The corresponding expected distortions on $\left\{S_{1, k}\right\}$ and on $\left\{S_{2, k}\right\}$ are

$$
\begin{aligned}
& \tilde{D}_{1}^{\mathrm{u}}(\alpha, \beta)=\sigma^{2} \frac{P^{2} \beta^{2}\left(1-\rho^{2}\right)+P N\left(\alpha^{2}+2 \rho \alpha \beta+\beta^{2}\left(2-\rho^{2}\right)\right)+N^{2}\left(\alpha^{2}+2 \rho \alpha \beta+\beta^{2}\right)}{(P+N)^{2}\left(\alpha^{2}+2 \rho \alpha \beta+\beta^{2}\right)}, \\
& \tilde{D}_{2}^{\mathrm{u}}(\alpha, \beta)=\sigma^{2} \frac{P^{2} \alpha^{2}\left(1-\rho^{2}\right)+P N\left(\beta^{2}+2 \rho \alpha \beta+\alpha^{2}\left(2-\rho^{2}\right)\right)+N^{2}\left(\alpha^{2}+2 \rho \alpha \beta+\beta^{2}\right)}{(P+N)^{2}\left(\alpha^{2}+2 \rho \alpha \beta+\beta^{2}\right)} .
\end{aligned}
$$

The optimality of this uncoded scheme below a certain SNR-threshold is stated next.

Proposition 3.1. Let $\left(D_{1}, D_{2}\right)$ be an achievable distortion pair for our point-to-point setting. If

$$
\frac{P}{N} \leq \Gamma\left(D_{1}, \sigma^{2}, \rho\right)
$$

where the threshold $\Gamma$ is given by

$$
\Gamma\left(D_{1}, \sigma^{2}, \rho\right)= \begin{cases}\frac{\sigma^{4}\left(1-\rho^{2}\right)-2 D_{1} \sigma^{2}\left(1-\rho^{2}\right)+D_{1}^{2}}{D_{1}\left(\sigma^{2}\left(1-\rho^{2}\right)-D_{1}\right)} & \text { if } 0<D_{1}<\sigma^{2}\left(1-\rho^{2}\right), \\ +\infty & \text { else }\end{cases}
$$

then there exist $\alpha^{*}, \beta^{*} \geq 0$ such that

$$
\tilde{D}_{1}^{\mathrm{u}}\left(\alpha^{*}, \beta^{*}\right) \leq D_{1} \quad \text { and } \quad \tilde{D}_{2}^{\mathrm{u}}\left(\alpha^{*}, \beta^{*}\right) \leq D_{2} .
$$

Proof. See Appendix B.

In the symmetric case, Proposition 3.1 simplifies as follows.

Corollary 3.1. Let $D>0$ be such that $(D, D)$ is an achievable distortion pair for the point-to-point problem. If

$$
\frac{P}{N} \leq \frac{2 \rho}{1-\rho}
$$

then the pair $(D, D)$ is achieved by the uncoded scheme with time-k channel input

$$
X_{k}^{\mathrm{u}}(\alpha, \alpha)=\sqrt{\frac{P}{2 \sigma^{2}(1+\rho)}}\left(S_{1, k}+S_{2, k}\right) \quad \text { for } k \in\{1,2, \ldots, n\} .
$$

Corollary 3.1 can also be verified without relying on Proposition 3.1. This is discussed in the following remark.

Remark 3.2. The distortions resulting from the uncoded scheme with any choice of $(\alpha, \beta)$ such that $\alpha=\beta$ are

$$
\tilde{D}_{i}^{\mathrm{u}}(\alpha, \alpha)=\sigma^{2} \frac{P(1-\rho)+2 N}{2(P+N)} \quad i \in\{1,2\} .
$$

By evaluating the necessary and sufficient condition of (9) for the case where $D_{1}=$ $D_{2}=D$, it follows that this is indeed the minimal achievable distortion for all $P / N$ satisfying (13).

This concludes our discussion of the point-to-point problem. 


\section{Main Results}

\subsection{Necessary Condition for Achievability of $\left(D_{1}, D_{2}\right)$}

Theorem 4.1. A necessary condition for the achievability of a distortion pair $\left(D_{1}, D_{2}\right)$ is that

$$
R_{S_{1}, S_{2}}\left(D_{1}, D_{2}\right) \leq \frac{1}{2} \log _{2}\left(1+\frac{P_{1}+P_{2}+2 \rho \sqrt{P_{1} P_{2}}}{N}\right) .
$$

Proof. See Appendix C.

Remark 4.1. Theorem 4.1 can be extended to a wider class of sources and distortion measures. Indeed, if the source is any memoryless bi-variate source (not necessarily zero-mean Gaussian) and if the fidelity measures $d_{1}\left(s_{1}, \hat{s}_{1}\right), d_{2}\left(s_{2}, \hat{s}_{2}\right) \geq 0$ that are used to measure the distortion in reconstructing each of the source components are arbitrary, then the pair $\left(D_{1}, D_{2}\right)$ is achievable with powers $P_{1}, P_{2}$ only if

$$
\inf _{\substack{P_{\widehat{S}_{1}, \widehat{S}_{2} \mid S_{1}, S_{2}} \\ \mathrm{E}\left[d_{1}\left(S_{1}, \hat{S}_{1}\right)\right] \leq D_{1} \\ \mathrm{E}\left[d_{2}\left(S_{2}, \hat{S}_{2}\right)\right] \leq D_{2}}} I\left(S_{1}, S_{2} ; \widehat{S}_{1}, \widehat{S}_{2}\right) \leq \frac{1}{2} \log \left(1+\frac{P_{1}+P_{2}+2 \rho_{\max } \sqrt{P_{1} P_{2}}}{N}\right),
$$

where $\rho_{\max }$ is the Hirschfeld-Gebelein-Rényi maximal correlation between $S_{1}$ and $S_{2}$, i.e.

$$
\rho_{\max }=\sup \mathrm{E}\left[g\left(S_{1}\right) h\left(S_{2}\right)\right]
$$

where the supremum is over all functions $g(\cdot), h(\cdot)$ under which

$$
\mathrm{E}\left[g\left(S_{1}\right)\right]=\mathrm{E}\left[h\left(S_{2}\right)\right]=0 \quad \text { and } \quad \mathrm{E}\left[g^{2}\left(S_{1}\right)\right]=\mathrm{E}\left[h^{2}\left(S_{2}\right)\right]=1 .
$$

For the bi-variate Gaussian memoryless source, condition (15) reduces to (14) because in this case $\rho_{\max }$ is equal to $\rho$ [15, Lemma 10.2, p. 182].

Remark 4.2. The necessary condition of Theorem 4.1 corresponds to the necessary and sufficient condition for the achievability of a distortion pair $\left(D_{1}, D_{2}\right)$ when the source $\left\{\left(S_{1, k}, S_{2, k}\right)\right\}$ is transmitted over a point-to-point AWGN channel of input power constraint $P_{1}+P_{2}+\rho \sqrt{P_{1} P_{2}}$ (see (9) ). This relation is not a coincidence. The proof of Theorem 4.1 (see Appendix $[$ C) indeed consists of reducing the multiple-access problem to the problem of transmitting the source $\left\{\left(S_{1, k}, S_{2, k}\right)\right\}$ over an AWGN channel of input power constraint $P_{1}+P_{2}+\rho \sqrt{P_{1} P_{2}}$.

We now specialize Theorem 4.1 to the symmetric case. We combine the explicit form of the rate-distortion function in (10) with (14) and substitute $(D, D)$ for $\left(D_{1}, D_{2}\right)$ to obtain:

Corollary 4.1. In the symmetric case

$$
D^{*}\left(\sigma^{2}, \rho, P, N\right) \geq \begin{cases}\sigma^{2} \frac{P\left(1-\rho^{2}\right)+N}{2 P(1+\rho)+N} & \text { for } \frac{P}{N} \in\left(0, \frac{\rho}{1-\rho^{2}}\right] \\ \sigma^{2} \sqrt{\frac{\left(1-\rho^{2}\right) N}{2 P(1+\rho)+N}} & \text { for } \frac{P}{N}>\frac{\rho}{1-\rho^{2}} .\end{cases}
$$


Corollary 4.1 concludes the section on the necessary condition for the achievability of a distortion pair $\left(D_{1}, D_{2}\right)$. We now compare this necessary condition to several sufficient conditions. The first sufficient condition that we consider is based on conventional source-channel separation.

\subsection{Source-Channel Separation}

As a benchmark we now consider the set of distortion pairs that are achieved by combining the optimal scheme for the corresponding source-coding problem with the optimal scheme for the corresponding channel-coding problem.

The corresponding source-coding problem is illustrated in Figure 4, The two source

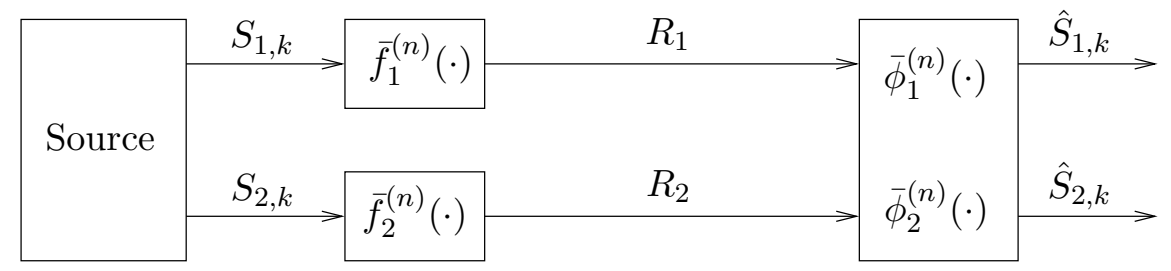

Figure 4: Distributed source coding problem for a bi-variate Gaussian source.

components are observed by two separate encoders. These two encoders wish to describe their source sequence to the common receiver by means of individual rate-limited and error-free bit pipes. The receiver estimates each of the sequences subject to expected squared-error distortion. A detailed description of this problem can be found in [5, 6]. The associated rate-distortion region is given in the next theorem.

Theorem 4.2 (Oohama [5]; Wagner, Tavildar, Viswanath [6]). For the Gaussian twoterminal source coding problem (with source components of unit variances) a distortionpair $\left(D_{1}, D_{2}\right)$ is achievable if, and only if,

$$
\left(R_{1}, R_{2}\right) \in \mathcal{R}_{1}\left(D_{1}\right) \cap \mathcal{R}_{2}\left(D_{2}\right) \cap \mathcal{R}_{\text {sum }}\left(D_{1}, D_{2}\right),
$$

where

$$
\begin{array}{r}
\mathcal{R}_{1}\left(D_{1}\right)=\left\{\left(R_{1}, R_{2}\right): R_{1} \geq \frac{1}{2} \log _{2}^{+}\left[\frac{1}{D_{1}}\left(1-\rho^{2}\left(1-2^{-2 R_{2}}\right)\right)\right]\right\} \\
\mathcal{R}_{2}\left(D_{2}\right)=\left\{\left(R_{1}, R_{2}\right): R_{2} \geq \frac{1}{2} \log _{2}^{+}\left[\frac{1}{D_{2}}\left(1-\rho^{2}\left(1-2^{-2 R_{1}}\right)\right)\right]\right\} \\
\mathcal{R}_{\text {sum }}\left(D_{1}, D_{2}\right)=\left\{\left(R_{1}, R_{2}\right): R_{1}+R_{2} \geq \frac{1}{2} \log _{2}^{+}\left[\frac{\left(1-\rho^{2}\right) \beta\left(D_{1}, D_{2}\right)}{2 D_{1} D_{2}}\right]\right\}
\end{array}
$$

with

$$
\beta\left(D_{1}, D_{2}\right)=1+\sqrt{1+\frac{4 \rho^{2} D_{1} D_{2}}{\left(1-\rho^{2}\right)^{2}}} .
$$

The distortions achievable by source-channel separation now follow from combining Theorem 4.2 with the capacity of the Gaussian multiple-access channel (see e.g. [16, 17]). We state here the explicit expression for the resulting distortion pairs only for the symmetric case. 
Corollary 4.2. In the symmetric case, a distortion $D$ is achievable by source-channel separation if, and only if,

$$
D \geq \sigma^{2} \frac{\sqrt{N\left(N+2 P\left(1-\rho^{2}\right)\right)}}{2 P+N} .
$$

We next consider several combined source-channel coding schemes. The first scheme is an uncoded scheme.

\subsection{Uncoded Scheme}

In this section we consider an uncoded transmission scheme, which, as we show, is optimal below a certain SNR-threshold.

The uncoded scheme operates as follows. At every time instant $k$, Encoder $i \in$ $\{1,2\}$ produces as channel input $X_{i, k}$ a scaled version of the time- $k$ source output $S_{i, k}$. The corresponding scaling is such that the average power constraint of the channel is satisfied. That is,

$$
X_{i, k}^{\mathrm{u}}=\sqrt{\frac{P_{i}}{\sigma^{2}}} S_{i, k} \quad k \in\{1,2, \ldots, n\} .
$$

Based on the resulting time- $k$ channel output $Y_{k}$, the decoder then performs an MMSE estimate $\hat{S}_{i, k}^{\mathrm{u}}$ of the source output $S_{i, k}, i \in\{1,2\}, k \in\{1,2, \ldots, n\}$. That is,

$$
\hat{S}_{i, k}^{\mathrm{u}}=\mathrm{E}\left[S_{i, k} \mid Y_{k}\right] \quad k \in\{1,2, \ldots, n\} .
$$

The expected distortions $\left(D_{1}^{\mathrm{u}}, D_{2}^{\mathrm{u}}\right)$ resulting from this uncoded scheme as well as the optimality of the scheme below a certain SNR-threshold are given in the following theorem.

Theorem 4.3. The distortion pairs $\left(D_{1}^{\mathrm{u}}, D_{2}^{\mathrm{u}}\right)$ resulting from the described uncoded scheme are given by

$$
D_{1}^{\mathrm{u}}=\sigma^{2} \frac{\left(1-\rho^{2}\right) P_{2}+N}{P_{1}+P_{2}+2 \rho \sqrt{P_{1} P_{2}}+N} \quad D_{2}^{\mathrm{u}}=\sigma^{2} \frac{\left(1-\rho^{2}\right) P_{1}+N}{P_{1}+P_{2}+2 \rho \sqrt{P_{1} P_{2}}+N} .
$$

These distortion pairs are optimal, i.e., lie on the boundary of the distortion region, whenever

$$
P_{2}\left(1-\rho^{2}\right)^{2}\left(P_{1}+2 \rho \sqrt{P_{1} P_{2}}\right) \leq N \rho^{2}\left(2 P_{2}\left(1-\rho^{2}\right)+N\right) .
$$

Proof. The evaluation of $\left(D_{1}^{\mathrm{u}}, D_{2}^{\mathrm{u}}\right)$ leading to (18) is given in Appendix D. Based on the expressions for $D_{1}^{\mathrm{u}}$ and $D_{2}^{\mathrm{u}}$ the optimality of the uncoded scheme now follows from verifying that for all $P_{1}, P_{2}$ and $N$ satisfying (19) the corresponding distortion pair $\left(D_{1}^{\mathrm{u}}, D_{2}^{\mathrm{u}}\right)$ satisfies the necessary condition (14) of Theorem 4.1 with equality. To verify this, one can first verify that for all $P_{1}, P_{2}$ and $N$ satisfying (19) we have $\left(D_{1}^{\mathrm{u}}, D_{2}^{\mathrm{u}}\right) \in$ $\mathscr{D}_{3}$. 
Remark 4.3. The optimality of the uncoded scheme can also be derived in a more conceptual way. To see this, denote by $\mathscr{D}_{\mathrm{MAC}}\left(\sigma^{2}, \rho, P_{1}, P_{2}, N\right)$ the distortion region for our multiple-access problem, and by $\mathscr{D}_{\mathrm{PTP}}\left(\sigma^{2}, \rho, P, N\right)$ the distortion region for the point-to-point problem of Section 3. The optimality of the uncoded scheme for the multiple-access problem now follows from combining the following three statements:

A)

$$
\mathscr{D}_{\mathrm{MAC}}\left(\sigma^{2}, \rho, P_{1}, P_{2}, N\right) \subseteq \mathscr{D}_{\mathrm{PTP}}\left(\sigma^{2}, \rho, P_{1}+P_{2}+2 \rho \sqrt{P_{1} P_{2}}, N\right)
$$

Statement A) is nothing but a restatement of Theorem 4.1 and Remark 4.2 .

B) For the point-to-point problem of Section 3 with power constraint $P=P_{1}+P_{2}+$ $2 \rho \sqrt{P_{1} P_{2}}$, let $\left(D_{1}, D_{2}\right)$ be a distorion pair resulting from the uncoded scheme of Section 3.3. If

$$
\frac{P_{1}+P_{2}+2 \rho \sqrt{P_{1} P_{2}}}{N} \leq \Gamma\left(D_{1}, \sigma^{2}, \rho\right),
$$

where $\Gamma$ is the threshold function defined in (12), then $\left(D_{1}, D_{2}\right)$ lies on the boundary of $\mathscr{D}_{\mathrm{PTP}}\left(\sigma^{2}, \rho, P_{1}+P_{2}+2 \rho \sqrt{P_{1} P_{2}}, N\right)$.

Statement B) follows immediately by Proposition 3.1

C) Let $\left(\tilde{D}_{1}^{\mathrm{u}}(\alpha, \beta), \tilde{D}_{2}^{\mathrm{u}}(\alpha, \beta)\right)$ be the distortion pair resulting from the uncoded scheme for the point-to-point problem, and let $\left(D_{1}^{\mathrm{u}}, D_{2}^{\mathrm{u}}\right)$ be the distortion pair resulting from the uncoded scheme for the multiple-access problem. Then, if

then

$$
\alpha=\sqrt{\frac{P_{1}}{\sigma^{2}}} \quad \text { and } \quad \beta=\sqrt{\frac{P_{2}}{\sigma^{2}}},
$$

$$
\left(\tilde{D}_{1}^{\mathrm{u}}(\alpha, \beta), \tilde{D}_{2}^{\mathrm{u}}(\alpha, \beta)\right)=\left(D_{1}^{\mathrm{u}}, D_{2}^{\mathrm{u}}\right)
$$

Statement C) follows since in the multiple-access problem, the channel output

$$
Y_{k}=\alpha S_{1, k}+\beta S_{2, k}+Z_{k},
$$

resulting from the uncoded scheme mimics the channel output of the uncoded scheme for the point-to-point problem with power constraint $P=P_{1}+P_{2}+2 \rho \sqrt{P_{1} P_{2}}$. Thus, while in the multiple-access problem the encoders cannot cooperate, the channel performs the addition for them. And since the reconstructors are the same in the multiple-access problem and the point-to-point problem, the resulting distortions are the same in both problems.

Combining Statements A), B) and C), gives that if

$$
\frac{P_{1}+P_{2}+2 \rho \sqrt{P_{1} P_{2}}}{N} \leq \Gamma\left(D_{1}^{\mathrm{u}}, \sigma^{2}, \rho\right),
$$


then $\left(D_{1}^{\mathrm{u}}, D_{2}^{\mathrm{u}}\right)$ lies on the boundary of $\mathscr{D}_{\mathrm{MAC}}\left(\sigma^{2}, \rho, P_{1}, P_{2}, N\right)$, i.e., the uncoded scheme for the multiple-access problem is optimal. The threshold condition (19) now follows by (20) and from substituting therein the value of $D_{1}^{\mathrm{u}}$ by its explicit expression given in (18).

As a special case of Theorem 4.3 we obtain:

Corollary 4.3. In the symmetric case,

$$
D^{*}\left(\sigma^{2}, \rho, P, N\right)=\sigma^{2} \frac{P\left(1-\rho^{2}\right)+N}{2 P(1+\rho)+N}, \quad \text { for all } \quad \frac{P}{N} \leq \frac{\rho}{1-\rho^{2}} .
$$

Moreover, for all SNRs below the given threshold, the minimal distortion $D^{*}\left(\sigma^{2}, \rho, P, N\right)$ is achieved by the uncoded scheme.

The upper and lower bounds that result on $D^{*}\left(\sigma^{2}, \rho, P, N\right)$ from our derived necessary conditions and sufficient conditions are illustrated in Figure 5 for a source of correlation coefficient $\rho=0.5$. For the SNRs below the threshold of (21) (marked by the

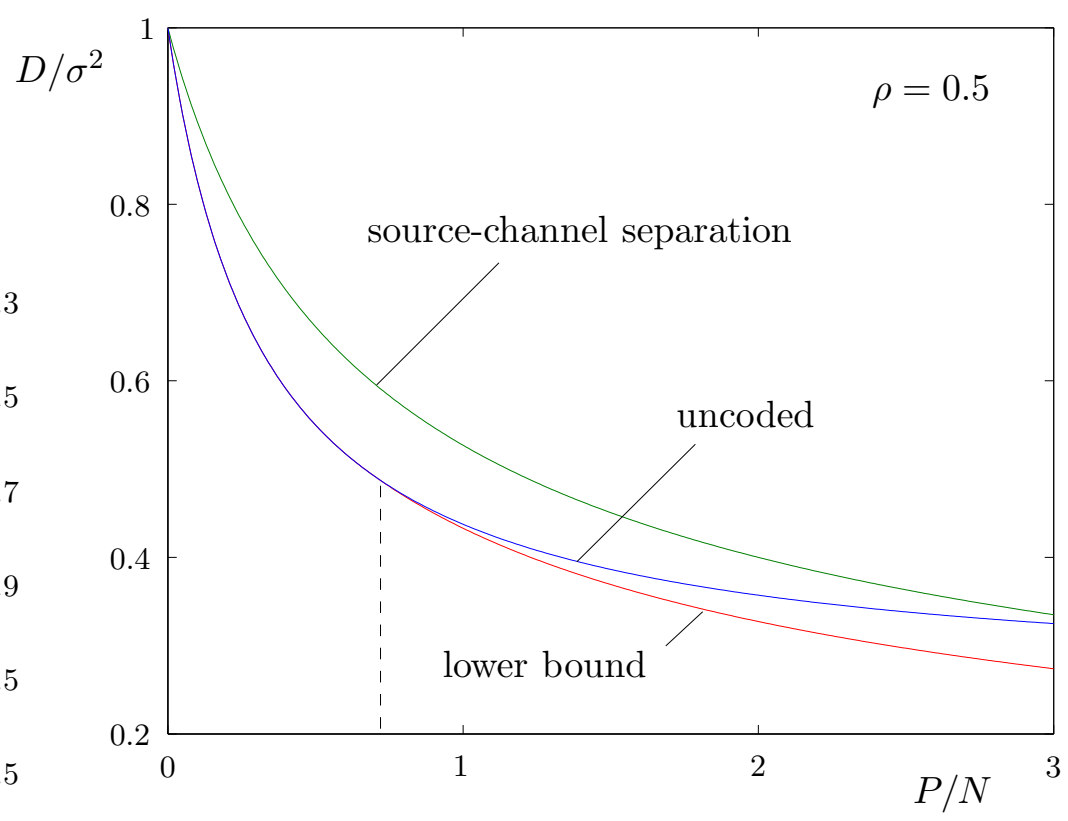

Figure 5: Upper and lower bounds on $D^{*}\left(\sigma^{2}, \rho, P, N\right)$ for a source of correlation coefficient $\rho=0.5$.

dashed line) the uncoded approach performs significantly better than the separationbased approach. However, for SNRs above the threshold of (21) the performance of the uncoded scheme gets successively worse. By the expressions in (21), we obtain that in the symmetric case

$$
\lim _{P / N \rightarrow \infty} D_{i}^{\mathrm{u}}=\sigma^{2} \frac{1-\rho}{2}, \quad i \in\{1,2\}
$$


That is, as $P / N \rightarrow \infty$ the distortion $D_{i}^{\mathrm{u}}$ does not tend to zero. The reason is that as the noise tends to zero, the channel output corresponding to the uncoded scheme tends to $\alpha \mathbf{S}_{1}+\beta \mathbf{S}_{2}$, from which $\mathbf{S}_{1}$ and $\mathbf{S}_{2}$ cannot be recovered.

\subsection{Vector-Quantizer Scheme}

In this section, we propose a coding scheme that improves on the uncoded scheme at high SNR. In this scheme the signal transmitted by each encoder is a vector-quantized version of its source sequence. The vital difference to the separation-based scheme is that the vector-quantized sequences are not mapped to bits before they are transmitted. Instead, the vector-quantized sequences are the channel inputs themselves. This transfers some of the correlation from the source to the channel inputs with the channel inputs still being from discrete sets, thereby enabling the decoder to make distinct estimates of $\mathbf{S}_{1}$ and of $\mathbf{S}_{2}$. For this scheme, we derive the achievable distortions and, based on those and on the necessary condition of Theorem 4.1, deduce the high SNR asymptotics of an optimal scheme.

The structure of an encoder of our scheme is illustrated in Figure 6. First, the

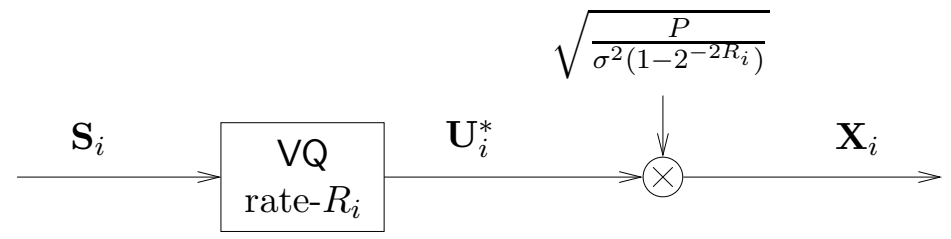

Figure 6: Encoder of Vector-Quantizer Scheme.

source sequence $\mathbf{S}_{i}$ is quantized by an optimal rate- $R_{i}$ vector-quantizer. The resulting quantized sequence is denoted by $\mathbf{U}_{i}^{*}$. For its transmission over the channel, this sequence is scaled so as to satisfy the average power constraint of (4). That is, the channel input sequence $\mathbf{X}_{i}$ is given by

$$
\mathbf{X}_{i}=\sqrt{\frac{P}{\sigma^{2}\left(1-2^{-2 R_{i}}\right)}} \mathbf{U}_{i}^{*} \quad i \in\{1,2\} .
$$

Based on the channel output $\mathbf{Y}$ resulting from $\mathbf{X}_{1}$ and $\mathbf{X}_{2}$, the decoder then estimates the two source sequences $\mathbf{S}_{1}$ and $\mathbf{S}_{2}$. It does this in two steps. First, it tries to recover the two transmitted sequences $\mathbf{U}_{1}^{*}$ and $\mathbf{U}_{2}^{*}$ from the channel output sequence $\mathbf{Y}$ by performing joint decoding that takes into consideration the correlation between two transmitted sequences $\mathbf{U}_{1}^{*}$ and $\mathbf{U}_{2}^{*}$. The resulting decoded sequences are denoted by $\hat{\mathbf{U}}_{1}$ and $\hat{\mathbf{U}}_{2}$ respectively. In the second step, the decoder performs approximate MMSE estimates $\hat{\mathbf{S}}_{i}, i \in\{1,2\}$, of the source sequences $\mathbf{S}_{i}$ based on $\hat{\mathbf{U}}_{1}$ and $\hat{\mathbf{U}}_{2}$, i.e.

$$
\begin{aligned}
\hat{\mathbf{S}}_{i} & =\gamma_{i 1} \hat{\mathbf{U}}_{1}+\gamma_{i 2} \hat{\mathbf{U}}_{2}, \\
& \approx \mathrm{E}\left[\mathbf{S}_{i} \mid \hat{\mathbf{U}}_{1}, \hat{\mathbf{U}}_{2}\right] .
\end{aligned}
$$

A detailed description of the scheme is given in Appendix E.

The distortion pairs achieved by this vector-quantizer scheme are stated in the following theorem. 
Theorem 4.4. The distortions achieved by the vector-quantizer scheme are all pairs $\left(D_{1}, D_{2}\right)$ satisfying

$$
\begin{aligned}
& D_{1}>\sigma^{2} 2^{-2 R_{1}} \cdot \frac{1-\rho^{2}\left(1-2^{-2 R_{2}}\right)}{1-\tilde{\rho}^{2}} \\
& D_{2}>\sigma^{2} 2^{-2 R_{2}} \cdot \frac{1-\rho^{2}\left(1-2^{-2 R_{1}}\right)}{1-\tilde{\rho}^{2}},
\end{aligned}
$$

where the rate-pair $\left(R_{1}, R_{2}\right)$ satisfies

$$
\begin{aligned}
R_{1} & <\frac{1}{2} \log _{2}\left(\frac{P_{1}\left(1-\tilde{\rho}^{2}\right)+N}{N\left(1-\tilde{\rho}^{2}\right)}\right) \\
R_{2} & <\frac{1}{2} \log _{2}\left(\frac{P_{2}\left(1-\tilde{\rho}^{2}\right)+N}{N\left(1-\tilde{\rho}^{2}\right)}\right) \\
R_{1}+R_{2} & <\frac{1}{2} \log _{2}\left(\frac{P_{1}+P_{2}+2 \tilde{\rho} \sqrt{P_{1} P_{2}}+N}{N\left(1-\tilde{\rho}^{2}\right)}\right),
\end{aligned}
$$

and where

$$
\tilde{\rho}=\rho \sqrt{\left(1-2^{-2 R_{1}}\right)\left(1-2^{-2 R_{2}}\right)} .
$$

Proof. See Appendix E,

Remark 4.4. The coefficient $\tilde{\rho}$ corresponds to the asymptotic average correlation coefficient between two time- $k$ channel inputs $X_{1, k}$ and $X_{2, k}$.

Based on Theorem 4.4 we now derive two more results: we show that for the symmetric version of our problem, source-channel separation is suboptimal also at high $\mathrm{SNR}$, and we determine the precise high-SNR asymptotics of an optimal scheme. We begin with the sub-optimality of source-channel separation. To this end, we restate Theorem 4.4 more specifically for the symmetric case.

Corollary 4.4. In the symmetric case

$$
D^{*}\left(\sigma^{2}, \rho, P, N\right) \leq \sigma^{2} 2^{-2 R} \cdot \frac{1-\rho^{2}\left(1-2^{-2 R}\right)}{1-\rho^{2}\left(1-2^{-2 R}\right)^{2}},
$$

where

$$
R<\frac{1}{4} \log _{2}\left(\frac{2 P\left(1+\rho\left(1-2^{-2 R}\right)\right)+N}{N\left(1-\rho^{2}\left(1-2^{-2 R}\right)^{2}\right)}\right) .
$$

By comparing the achievable distortion of the vector-quantizer scheme, in Corollary 4.4, with the achievable distortion of the separation-based scheme, in Corollary 4.2, we obtain:

Corollary 4.5. In the symmetric case with $\rho>0$, source-channel separation is suboptimal for all $P>0$. 
We turn to the high-SNR asymptotics of an optimal scheme. To this end, let $\left(D_{1}^{*}, D_{2}^{*}\right)$ denote an arbitrary distortion pair resulting from an optimal scheme. For a subset of those distortion pairs, the high SNR behavior is described in the following theorem.

Theorem 4.5 (High-SNR Distortion). The high-SNR asymptotic behavior of $\left(D_{1}^{*}, D_{2}^{*}\right)$ is given by

$$
\lim _{N \rightarrow 0} \frac{P_{1}+P_{2}+2 \rho \sqrt{P_{1} P_{2}}}{N} D_{1}^{*} D_{2}^{*}=\sigma^{4}\left(1-\rho^{2}\right),
$$

provided that $D_{1}^{*} \leq \sigma^{2}$ and $D_{2}^{*} \leq \sigma^{2}$, and that

$$
\lim _{N \rightarrow 0} \frac{N}{P_{1} D_{1}^{*}}=0 \quad \text { and } \quad \lim _{N \rightarrow 0} \frac{N}{P_{2} D_{2}^{*}}=0 .
$$

Proof. See Appendix E.

We restate Theorem 4.5 more specifically for the symmetric case. Since there $D_{1}^{*}=$ $D_{2}^{*}=D^{*}\left(\sigma^{2}, \rho, P, N\right)$, condition (27) is implicitly satisfied. Thus,

Corollary 4.6. In the symmetric case

$$
\lim _{P / N \rightarrow \infty} \sqrt{\frac{P}{N}} D^{*}\left(\sigma^{2}, \rho, P, N\right)=\sigma^{2} \sqrt{\frac{1-\rho}{2}} .
$$

Remark 4.5. Corollary 4.6 can also be deduced without Theorem 4.5, by comparing the distortion of the vector-quantizer scheme in Corollary 4.4 to the lower bound on $D^{*}\left(\sigma^{2}, \rho, P, N\right)$ in Corollary 4.1.

To get some understanding of the coefficient on the RHS of (28), let us first rewrite (28) as follows

$$
D^{*}\left(\sigma^{2}, \rho, P, N\right) \approx \sigma^{2} \sqrt{\frac{N\left(1-\rho^{2}\right)}{2 P(1+\rho)}} \quad \text { as } \quad \frac{P}{N} \gg 1 .
$$

Next, let us compare this asymptotic behavior to that of two suboptimal schemes: the best separation-based scheme and the suboptimal separation-based scheme that completely ignores the source correlation, i.e., the best scheme where the transmitters and the receiver treat the two source components as if they where independent. Denoting the distortion of the best separation-based scheme by $D_{\mathrm{SB}}$ and the distortion of the scheme that ignores the source correlation by $D_{\mathrm{IC}}$, gives

$$
D_{\mathrm{SB}} \approx \sigma^{2} \sqrt{\frac{N\left(1-\rho^{2}\right)}{2 P}} \quad \text { and } \quad D_{\mathrm{IC}} \approx \sigma^{2} \sqrt{\frac{N}{2 P}}, \quad \text { as } \quad \frac{P}{N} \gg 1 .
$$

The asymptotic expression for $D_{\mathrm{SB}}$ follows by Corollary 4.2 and the asymptotic expression for $D_{\text {IC }}$ follows from combining the rate-distortion function of a Gaussian random variable, see e.g. [18, Theorem 13.3.2, p. 344], with the capacity region of the Gaussian multiple-access channel, see e.g. [18, Section 14.3.6, p. 403]. 
The asymptotic behavior can now be understood as follows. The denominator under the square-root corresponds to the average power that the scheme under discussion produces on the sum of the channel inputs $X_{1, k}+X_{2, k}$. In the two separation-based approaches this average power is $2 P$, and in the vector-quantizer scheme this average power is $2 P(1+\rho)$ as $P / N \rightarrow \infty$. The numerator under the square-root consists of the noise variance $N$ multiplied by a coefficient reflecting the gain due to the logical exploitation of the source correlation. For the scheme ignoring the source correlation this coefficient is, by definition of the scheme, equal to 1, i.e., no gain, whereas for the best separation-based scheme and for the vector-quantizer scheme this coefficient is equal to $1-\rho^{2}$. The means by which this gain is obtained in the best separation-based scheme and in the vector-quantizer scheme are fundamentally different. In the separationbased scheme the gain is achieved by a generalized form of Slepian-Wolf coding (see [5]), whereas in the vector-quantizer scheme the gain is achieved by joint-typicality decoding that takes into consideration the correlation between the transmitted sequences $\mathbf{U}_{1}^{*}$ and $\mathbf{U}_{2}^{*}$ (see Theorem 4.4). The corresponding advantage of the vector-quantizer scheme is that by performing the logical exploitation only at the receiver, it additionally allows for exploiting the source correlation in a physical way, i.e., by producing a power boost in the transmitted signal pair.

\subsection{Superposition Approach}

The last scheme of this paper is a combination of the previously considered uncoded scheme and vector-quantizer scheme. One way to combine these schemes would be by time- and power-sharing. As stated in Remark 2.1, this would result in a convexification of the union of the achievable distortions of the two individual schemes. In this section, we instead propose an approach where the two schemes are superimposed. In the symmetric case, this approach results in better performances than time- and powersharing, and for all SNRs, the resulting distortion is very close to the lower bound on $D^{*}\left(\sigma^{2}, \rho, P, N\right)$ of Corollary 4.1. We also point out that for the simpler problem of transmitting a univariate memoryless Gaussian source over a point-to-point AWGN channel subject to expected squared-error distortion, a similar superposition approach was shown in [19] to yield a continuum of optimal schemes.

The superimposed scheme can be described as follows. The channel input sequence $\mathbf{X}_{i}$ produced by Encoder $i, i \in\{1,2\}$, is a linear combination of the source sequence $\mathbf{S}_{i}$ and its rate- $R_{i}$ vector-quantized version $\mathbf{U}_{i}^{*}$. That is,

$$
\mathbf{X}_{i}=\alpha_{i} \mathbf{S}_{i}+\beta_{i} \mathbf{U}_{i}^{*},
$$

where the sequence $\mathbf{U}_{i}^{*}$ is obtained in exactly the same way as in the vector-quantizer scheme, and where the coefficients $\alpha_{i}$ and $\beta_{i}$ are chosen so that the sequence $\mathbf{X}_{i}$ satisfies the power constraint (44), and so that the receiver can, with high probability, recover the transmitted codeword pair $\left(\mathbf{U}_{1}^{*}, \mathbf{U}_{2}^{*}\right)$. As we shall see, these two conditions will be satisfied as long as $\alpha_{i}$ and $\beta_{i}$ satisfy to within some $\epsilon$ 's and $\delta$ 's

$$
\alpha_{i} \in\left[0, \frac{P_{i}}{\sigma^{2}}\right] \quad \beta_{i}=\sqrt{\frac{P_{i}-\alpha_{i}^{2} \sigma^{2} 2^{-2 R_{i}}}{\sigma^{2}\left(1-2^{-2 R_{i}}\right)}}-\alpha_{i} \quad i \in\{1,2\} .
$$

(For a precise statement see Appendix G). 
From the resulting channel output $\mathbf{Y}=\mathbf{X}_{1}+\mathbf{X}_{2}+\mathbf{Z}$, the decoder then makes a guess $\left(\hat{\mathbf{U}}_{1}, \hat{\mathbf{U}}_{2}\right)$ of the transmitted sequences $\left(\mathbf{U}_{1}^{*}, \mathbf{U}_{2}^{*}\right)$. This guess is obtained by joint typicality decoding that takes into consideration the correlation between $\mathbf{U}_{1}^{*}, \mathbf{U}_{2}^{*}, \mathbf{S}_{1}$ and $\mathbf{S}_{2}$. From the sequences $\hat{\mathbf{U}}_{1}, \hat{\mathbf{U}}_{2}$, and $\mathbf{Y}$, the decoder then computes approximate MMSE estimates $\hat{\mathbf{S}}_{1}$ and $\hat{\mathbf{S}}_{2}$ of the source sequences $\mathbf{S}_{1}$ and $\mathbf{S}_{2}$, i.e.,

$$
\hat{\mathbf{S}}_{i}=\gamma_{i 1} \hat{\mathbf{U}}_{1}+\gamma_{i 2} \hat{\mathbf{U}}_{2}+\gamma_{i 3} \mathbf{Y} \quad i \in\{1,2\},
$$

where the coefficients $\gamma_{i j}$ are chosen such that $\hat{\mathbf{S}}_{i} \approx \mathbf{E}\left[\mathbf{S}_{i} \mid \mathbf{Y}, \hat{\mathbf{U}}_{1}, \hat{\mathbf{U}}_{2}\right]$. To state the explicit form of coefficients $\gamma_{i j}$, define for any rate pair $\left(R_{1}, R_{2}\right)$, where $R_{i} \geq 0$, the $3 \times 3$ matrix $\mathrm{K}\left(R_{1}, R_{2}\right)$ by

$$
\mathrm{K}\left(R_{1}, R_{2}\right) \triangleq\left(\begin{array}{lll}
\mathrm{k}_{11} & \mathrm{k}_{12} & \mathrm{k}_{13} \\
\mathrm{k}_{12} & \mathrm{k}_{22} & \mathrm{k}_{23} \\
\mathrm{k}_{13} & \mathrm{k}_{23} & \mathrm{k}_{33}
\end{array}\right)
$$

where

$$
\begin{aligned}
\mathrm{k}_{11}= & \sigma^{2}\left(1-2^{-2 R_{1}}\right) \\
\mathrm{k}_{12}= & \sigma^{2} \rho\left(1-2^{-2 R_{1}}\right)\left(1-2^{-2 R_{2}}\right) \\
\mathrm{k}_{13}= & \left(\alpha_{1}+\beta_{1}+\alpha_{2} \rho\right) \mathrm{k}_{11}+\beta_{2} \mathrm{k}_{12} \\
\mathrm{k}_{22}= & \sigma^{2}\left(1-2^{-2 R_{2}}\right) \\
\mathrm{k}_{23}= & \left(\alpha_{2}+\beta_{2}+\alpha_{1} \rho\right) \mathrm{k}_{22}+\beta_{1} \mathrm{k}_{12} \\
\mathrm{k}_{33}= & \alpha_{1}^{2} \sigma^{2}+2 \alpha_{1} \beta_{1} \mathrm{k}_{11}+2 \alpha_{1} \alpha_{2} \rho \sigma^{2}+2 \alpha_{1} \beta_{2} \rho \mathrm{k}_{22}+\beta_{1}^{2} \mathrm{k}_{11}+2 \beta_{1} \alpha_{2} \rho \mathrm{k}_{11} \\
& +2 \beta_{1} \beta_{2} \mathrm{k}_{12}+2 \alpha_{2} \beta_{2} \mathrm{k}_{22}+\alpha_{2}^{2} \sigma^{2}+\beta_{2}^{2} \mathrm{k}_{22}+N .
\end{aligned}
$$

The coefficients $\gamma_{i j}$ are then given by

$$
\left(\begin{array}{c}
\gamma_{i 1} \\
\gamma_{i 2} \\
\gamma_{i 3}
\end{array}\right) \triangleq \mathrm{K}^{-1}\left(R_{1}, R_{2}\right)\left(\begin{array}{c}
\mathrm{c}_{i 1} \\
\mathrm{c}_{i 2} \\
\mathrm{c}_{i 3}
\end{array}\right) \quad i \in\{1,2\},
$$

where

$$
\begin{aligned}
& \mathrm{c}_{11}=\mathrm{k}_{11} \\
& \mathrm{c}_{12}=\rho \mathrm{k}_{22} \\
& \mathrm{c}_{13}=\left(\alpha_{1}+\alpha_{2} \rho\right) \sigma^{2}+\beta_{1} \mathrm{k}_{11}+\beta_{2} \rho \mathrm{k}_{22} \\
& \mathrm{c}_{21}=\rho \mathrm{k}_{11} \\
& \mathrm{c}_{22}=\mathrm{k}_{22} \\
& \mathrm{c}_{23}=\left(\alpha_{2}+\alpha_{1} \rho\right) \sigma^{2}+\beta_{1} \rho \mathrm{k}_{11}+\beta_{2} \mathrm{k}_{22} .
\end{aligned}
$$

The distortions achieved by the superimposed scheme are now given in the following theorem.

Theorem 4.6. The distortions achieved by the superposition approach are all pairs $\left(D_{1}, D_{2}\right)$ satisfying

$$
D_{i}>\sigma^{2}-\gamma_{i 1} c_{i 1}-\gamma_{i 2} c_{i 2}-\gamma_{i 3} c_{i 3} \quad i \in\{1,2\} .
$$


where the rate-pair $\left(R_{1}, R_{2}\right)$ satisfies

$$
\begin{aligned}
R_{1} & <\frac{1}{2} \log _{2}\left(\frac{\beta_{1}^{\prime 2} \mathrm{k}_{11}\left(1-\tilde{\rho}^{2}\right)+N^{\prime}}{N^{\prime}\left(1-\tilde{\rho}^{2}\right)}\right) \\
R_{2} & <\frac{1}{2} \log _{2}\left(\frac{\beta_{2}^{\prime 2} \mathrm{k}_{22}\left(1-\tilde{\rho}^{2}\right)+N^{\prime}}{N^{\prime}\left(1-\tilde{\rho}^{2}\right)}\right) \\
R_{1}+R_{2} & <\frac{1}{2} \log _{2}\left(\frac{\beta_{1}^{\prime 2} \mathrm{k}_{11}+\beta_{2}^{\prime 2} \mathrm{k}_{22}+2 \tilde{\rho} \beta_{1}^{\prime} \beta_{2}^{\prime} \sqrt{\mathrm{k}_{11} \mathrm{k}_{22}}+N^{\prime}}{N^{\prime}\left(1-\tilde{\rho}^{2}\right)}\right),
\end{aligned}
$$

for some $\alpha_{1}, \alpha_{2}, \beta_{1}$, and $\beta_{2}$ satisfying (30) and where

$$
N^{\prime}=\alpha_{1}^{2} \nu_{1}+\alpha_{2}^{2} \nu_{2}+2 \alpha_{1} \alpha_{2} \nu_{3}+N,
$$

where

$$
\begin{aligned}
& \nu_{1}=\sigma^{2}-\left(1-a_{1} \tilde{\rho}\right)^{2} \mathrm{k}_{11}-2\left(1-a_{1} \tilde{\rho}\right) a_{1} \mathrm{k}_{12}-a_{1}^{2} \mathrm{k}_{22} \\
& \nu_{2}=\sigma^{2}-\left(1-a_{2} \tilde{\rho}\right)^{2} \mathrm{k}_{22}-2\left(1-a_{2} \tilde{\rho}\right) a_{2} \mathrm{k}_{12}-a_{2}^{2} \mathrm{k}_{11} \\
& \nu_{3}=\rho \sigma^{2}-\left(\left(1-a_{1} \tilde{\rho}\right)\left(1-a_{2} \tilde{\rho}\right)+a_{1} a_{2}\right) \mathrm{k}_{12}-\left(1-a_{1} \tilde{\rho}\right) a_{2} \mathrm{k}_{11}-\left(1-a_{2} \tilde{\rho}\right) a_{1} \mathrm{k}_{22},
\end{aligned}
$$

with

$$
\begin{aligned}
& \beta_{1}^{\prime}=\alpha_{1}\left(1-a_{1} \tilde{\rho}\right)+\beta_{1}+\alpha_{2} a_{2} \\
& \beta_{2}^{\prime}=\alpha_{2}\left(1-a_{2} \tilde{\rho}\right)+\beta_{2}+\alpha_{1} a_{1}
\end{aligned}
$$

and with

$$
\begin{aligned}
& a_{1}=\frac{\rho 2^{-2 R_{1}}\left(1-2^{-2 R_{2}}\right)}{\left(1-2^{-2 R_{2}}\right)-2 \tilde{\rho}^{2} \sqrt{\left(1-2^{-2 R_{1}}\right)\left(1-2^{-2 R_{2}}\right)}+\tilde{\rho}^{2}\left(1-2^{-2 R_{1}}\right)}, \\
& a_{2}=\frac{\rho 2^{-2 R_{2}}\left(1-2^{-2 R_{1}}\right)}{\left(1-2^{-2 R_{1}}\right)-2 \tilde{\rho}^{2} \sqrt{\left(1-2^{-2 R_{1}}\right)\left(1-2^{-2 R_{2}}\right)}+\tilde{\rho}^{2}\left(1-2^{-2 R_{2}}\right)} .
\end{aligned}
$$

Proof. See Appendix G.

In the symmetric case where $P_{1}=P_{2}=P, R_{1}=R_{2}=R$ and where $\alpha_{1}=\alpha_{2}=\alpha$ and $\beta_{1}=\beta_{2}=\beta$, the matrix $\mathrm{K}(R, R)$ and the coefficients $\gamma_{i j}$ reduce to

$$
\mathrm{K}(R, R)=\left(\begin{array}{lll}
\mathrm{k}_{1} & \mathrm{k}_{2} & \mathrm{k}_{3} \\
\mathrm{k}_{2} & \mathrm{k}_{1} & \mathrm{k}_{3} \\
\mathrm{k}_{3} & \mathrm{k}_{3} & \mathrm{k}_{4}
\end{array}\right) \quad \text { where } \begin{aligned}
& \mathrm{k}_{1}=\sigma^{2}\left(1-2^{-2 R}\right) \\
& \mathrm{k}_{2}=\sigma^{2} \rho\left(1-2^{-2 R}\right)^{2} \\
& \mathrm{k}_{3}=(\alpha+\beta+\alpha \rho) \mathrm{k}_{1}+\beta \mathrm{k}_{2} \\
& \mathrm{k}_{4}=2 \alpha \mathrm{c}_{3}+2 \beta \mathrm{k}_{3}+N,
\end{aligned}
$$

and

$$
\left(\begin{array}{l}
\gamma_{1} \\
\gamma_{2} \\
\gamma_{3}
\end{array}\right) \triangleq \mathrm{K}^{-1}(R, R)\left(\begin{array}{l}
\mathrm{c}_{1} \\
\mathrm{c}_{2} \\
\mathrm{c}_{3}
\end{array}\right) \quad \text { where } \begin{aligned}
& \mathrm{c}_{1}=\mathrm{k}_{1} \\
& \mathrm{c}_{2}=\rho \mathrm{k}_{1} \\
& \mathrm{c}_{3}=\left(\alpha \sigma^{2}+\beta \mathrm{k}_{1}\right)(1+\rho) .
\end{aligned}
$$

Thus, in the symmetric case Theorem 4.6 simplifies as follows. 
Corollary 4.7. With the superposition approach in the symmetric case we can achieve the distortion

$$
\inf \sigma^{2}-\gamma_{1} c_{1}-\gamma_{2} c_{2}-\gamma_{3} c_{3},
$$

where the infimum is over all rates $R$ satisfying

$$
R<\frac{1}{4} \log _{2}\left(\frac{2 \beta^{\prime 2} \mathrm{k}_{1}(1+\tilde{\rho})+N^{\prime}}{N^{\prime}\left(1-\tilde{\rho}^{2}\right)}\right),
$$

for some $\alpha$ and $\beta$ satisfying

$$
\alpha \in\left[0, \frac{P}{\sigma^{2}}\right] \quad \text { and } \quad \beta=\sqrt{\frac{P-\alpha^{2} \sigma^{2} 2^{-2 R}}{\sigma^{2}\left(1-2^{-2 R}\right)}}-\alpha,
$$

and where

$$
\beta^{\prime}=\alpha\left(1+\frac{\rho 2^{-2 R}}{1-\tilde{\rho}^{2}}(1-\tilde{\rho})\right)+\beta
$$

and

$$
N^{\prime}=2 \alpha^{2}\left(\nu_{1}+\nu_{3}\right)+N,
$$

with

$$
\nu_{1}=\sigma^{2} 2^{-2 R} \frac{1-\rho \tilde{\rho}}{1-\tilde{\rho}^{2}} \quad \nu_{3}=\sigma^{2} \rho \frac{2^{-4 R}}{1-\tilde{\rho}^{2}}
$$

To conclude our main results we have illustrated in Figure 7 all presented upper and lower bounds on $D^{*}\left(\sigma^{2}, \rho, P, N\right)$.

\section{Summary}

We studied the power versus distortion trade-off for the distributed transmission of a memoryless bi-variate Gaussian source over a two-to-one average-power limited Gaussian multiple-access channel. In this problem, each of two separate transmitters observes a different component of a memoryless bi-variate Gaussian source. The two transmitters then describe their source component to a common receiver via a Gaussian multiple-access channel with average-power constraints on each channel input sequences. From the output of the multiple-access channel, the receiver wishes to reconstruct each source component with the least possible expected squared-error distortion. Our interest was in characterizing the distortion pairs that are simultaneously achievable on the two source components. These pairs are a function of the power constraints and the variance of the additive channel noise, as well as of the source variance and of the correlation coefficient between the two source components.

We first considered a different (non-distributed) problem, which was the point-topoint analog of our multiple-access problem. More precisely, we studied the power versus distortion trade-off for the transmission of a memoryless bi-variate Gaussian source over the AWGN channel, subject to expected squared-error distortion on each 


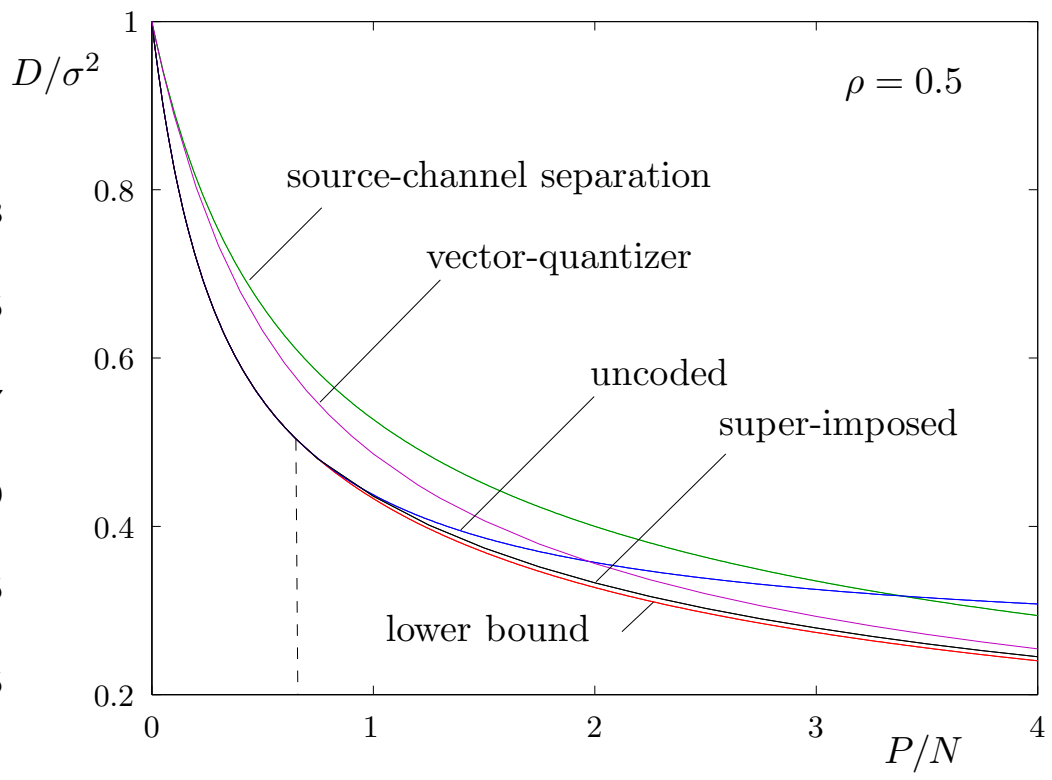

Figure 7: Upper and lower bounds on $D^{*}\left(\sigma^{2}, \rho, P, N\right)$ for a source of correlation coefficient $\rho=0.5$.

source component. For this problem, we determined the set of achievable distortion pairs by deriving the explicit expression for the rate-distortion function of a memoryless bi-variate Gaussian source. Moreover, we showed that below a certain SNR-threshold an uncoded transmission scheme is optimal.

For the multiple-access problem we then derived:

- A necessary condition for the achievability of a distortion pair (Theorem 4.1). This condition was obtained by reducing the multiple-access problem to a pointto-point problem. The key step was to upper bound the maximal correlation between two simultaneous channel inputs by using a result from maximum correlation theory.

- The optimality of an uncoded transmission scheme below a certain SNR-threshold (Theorem 4.3). In the symmetric case, this result becomes (Corollary 4.3)

$$
D^{*}\left(\sigma^{2}, \rho, P, N\right)=\sigma^{2} \frac{P\left(1-\rho^{2}\right)+N}{2 P(1+\rho)+N}, \quad \text { for all } \quad \frac{P}{N} \leq \frac{\rho}{1-\rho^{2}} .
$$

The strength of the underlying uncoded scheme is that it translates the entire source correlation onto the channel inputs, and thereby boosts the received power of the transmitted signal pair. Its weakness is that it allows the receiver to recover only the sum of the channel inputs.

- A sufficient condition based on a "vector-quantizer" scheme (Theorem 4.4). The motivation behind this scheme was to overcome the weakness of the uncoded 
scheme. To this end, rather than transmitting the source components in an uncoded manner, the scheme transmits a scaled version of the optimally vectorquantized source components (without channel coding).

- The precise high-SNR asymptotics of an optimal transmission scheme, which in the symmetric case are given by (Corollary 4.6)

$$
\lim _{P / N \rightarrow \infty} \sqrt{\frac{P}{N}} D^{*}\left(\sigma^{2}, \rho, P, N\right)=\sigma^{2} \sqrt{\frac{1-\rho}{2}} .
$$

This result follows from the "vector-quantizer" scheme (Theorem 4.4) and the necessary condition of Theorem 4.1

- The suboptimality, in the symmetric case, of source-channel separation at all SNRs. This follows from comparing the best separation-based approach (Corollary 4.2) with the achievable distortions from the "vector-quantizer" scheme (Corollary 4.4).

- A sufficient condition based on a superposition of the uncoded scheme and the vector-quantizer scheme (Theorem 4.6). In the symmetric case this superposition approach was shown to be optimal or close to optimal at all SNRs.

The presented sufficient conditions indicate that for the efficient exploitation of the source correlation it is necessary not only to exploit the source correlation in a logical way, e.g. by Slepian-Wolf-like strategies, but to additionally exploit the source correlation in a physical way. In the considered schemes, this is done by translating the source correlation onto the channel inputs. The logical exploitation of the source correlation is then performed at the receiver-side, e.g. by joint-typicality decoding taking into consideration the correlation between the channel inputs. 


\section{A Proof of Theorem 3.1}

Theorem 3.1 gives the expression of the rate-distortion function $R_{S_{1}, S_{2}}\left(D_{1}, D_{2}\right)$. A single-letter expression of this function, in the form of an optimization problem, follows from [20, Theorem 2, p. 856] and is

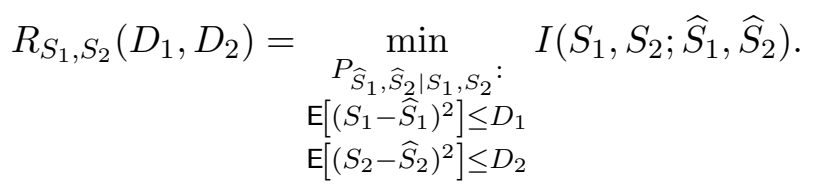

Thus, to prove Theorem 3.1 it remains to solve (40) for all distortion pairs $\left(D_{1}, D_{2}\right) \in$ $\left(0, \sigma^{2}\right] \times\left(0, \sigma^{2}\right]$. One way of doing this was presented in [14. Here, we present an alternative approach. The derivation is split in two parts. First we derive $R_{S_{1}, S_{2}}\left(D_{1}, D_{2}\right)$ for $\left(D_{1}, D_{2}\right) \in \mathscr{D}_{1}$, and then for $\left(D_{1}, D_{2}\right) \in\left(0, \sigma^{2}\right] \times\left(0, \sigma^{2}\right] \backslash \mathscr{D}_{1}$. Before starting with the derivations, we remark:

Remark A.1. The restriction to $\mathrm{E}\left[S_{1}^{2}\right]=\mathrm{E}\left[S_{2}^{2}\right]=\sigma^{2}$ and $\rho \in[0,1]$ incurs no loss in generality in the evaluation of $R_{S_{1}, S_{2}}\left(D_{1}, D_{2}\right)$ since the distortion region scales linearly with the source variance, and since the distortion region is the same for correlation coefficients $-\rho$ and $\rho$.

\section{A.1 $R_{S_{1}, S_{2}}\left(D_{1}, D_{2}\right)$ for the Region $\mathscr{D}_{1}$}

For pairs $\left(D_{1}, D_{2}\right)$ in $\mathscr{D}_{1}$ the evaluation of $R_{S_{1}, S_{2}}\left(D_{1}, D_{2}\right)$ is very simple. The pairs $\left(D_{1}, D_{2}\right) \in \mathscr{D}_{1}$ are pairs where the larger of the two distortions can be achieved from the description of the other source component without any additional information simply by exploiting the correlation between $S_{1}$ and $S_{2}$. For example, since $S_{2}=\left(S_{2}-\rho S_{1}\right)+\rho S_{1}$, from an optimally vector-quantized version of $S_{1}$ that yields a distortion $D_{1}$, a distortion $D_{2}=\sigma^{2}\left(1-\rho^{2}\right)+\rho^{2} D_{1}$ can be achieved on $S_{2}$ by picking the reconstruction $\hat{S}_{2}$ of $S_{2}$ as a scaled version of the reconstruction $\hat{S}_{1}$ of $S_{1}$. Thus, a necessary and sufficient condition to achieve a distortion pair $\left(D_{1}, \sigma^{2}\left(1-\rho^{2}\right)+\rho^{2} D_{1}\right)$ is

$$
R=\frac{1}{2} \log _{2}^{+}\left(\frac{\sigma^{2}}{D_{1}}\right) \text {. }
$$

By symmetry, the above argument also works when the roles of $S_{1}$ and $S_{2}$ reversed. Hence,

$$
\begin{aligned}
R_{S_{1}, S_{2}}\left(D_{1}, D_{2}\right) & =\max \left\{R_{S_{1}}\left(D_{1}\right), R_{S_{2}}\left(D_{2}\right)\right\} \\
& =\frac{1}{2} \log _{2}^{+}\left(\frac{\sigma^{2}}{D_{\min }}\right) \quad \text { if }\left(D_{1}, D_{2}\right) \in \mathscr{D}_{1} .
\end{aligned}
$$

\section{A.2 $R_{S_{1}, S_{2}}\left(D_{1}, D_{2}\right)$ for the Regions $\mathscr{D}_{2}$ and $\mathscr{D}_{3}$}

To solve (40) for $\left(D_{1}, D_{2}\right) \in\left(0, \sigma^{2}\right] \times\left(0, \sigma^{2}\right] \backslash \mathscr{D}_{1}$ we propose the following algorithm. First, we scale the source component $S_{2}$ by a factor $c \in \mathbb{R}^{+}$, where $\mathbb{R}^{+}$is the set of 
positive reals numbers, and then we decorrelate $\left(S_{1}, c S_{2}\right)$ to a pair $\left(V_{1}, V_{2}\right)$, by means of the unitary linear transformation:

$$
\left(\begin{array}{l}
V_{1} \\
V_{2}
\end{array}\right)=\left(\begin{array}{cc}
a_{1} & -a_{2} \\
a_{2} & a_{1}
\end{array}\right)\left(\begin{array}{ll}
1 & 0 \\
0 & c
\end{array}\right)\left(\begin{array}{l}
S_{1} \\
S_{2}
\end{array}\right)
$$

where $\left(a_{1}, a_{2}\right)^{\mathrm{T}},\left(-a_{2}, a_{1}\right)^{\mathrm{T}}$ are normalized eigenvectors of the covariance matrix of $\left(S_{1}, c S_{2}\right)$. Finally, we apply reverse waterfilling with rate $R$ on $\left(V_{1}, V_{2}\right)$, as described in [18, pp. 347]. The resulting reconstruction of $\left(V_{1}, V_{2}\right)$ is denoted by $\left(\hat{V}_{1}, \hat{V}_{2}\right)$. From $\left(\hat{V}_{1}, \hat{V}_{2}\right)$ the reconstruction $\left(\hat{S}_{1}, \hat{S}_{2}\right)$ of $\left(S_{1}, S_{2}\right)$ is then obtained by

$$
\left(\begin{array}{c}
\hat{S}_{1} \\
\hat{S}_{2}
\end{array}\right)=\mathrm{B}\left(\begin{array}{c}
\hat{V}_{1} \\
\hat{V}_{2}
\end{array}\right)
$$

where the matrix $B$ is the reverse transformation of (42), i.e.

$$
\mathrm{B}=\left(\begin{array}{cc}
1 & 0 \\
0 & \frac{1}{c}
\end{array}\right)\left(\begin{array}{cc}
a_{1} & a_{2} \\
-a_{2} & a_{1}
\end{array}\right)
$$

The probability law of $\left(S_{1}, S_{2}, \hat{S}_{1}, \hat{S}_{2}\right)$ that results from the algorithm is a solution to the optimization problem in (40). This is stated more precisely in the following theorem.

\section{Theorem A.1.}

i) For every distortion pair $\left(D_{1}, D_{2}\right) \in\left(0, \sigma^{2}\right] \times\left(0, \sigma^{2}\right] \backslash \mathscr{D}_{1}$ there exists some rate $R^{*} \in \mathbb{R}^{+}$and some scaling coefficient $c^{*} \in \mathbb{R}^{+}$such that the distortion pair resulting from the above algorithm is $\left(D_{1}, D_{2}\right)$.

ii) The probability law of $\left(S_{1}, S_{2}, \hat{S}_{1}, \hat{S}_{2}\right)$ resulting from this algorithm is a solution to the optimization problem in (40).

iii) For every $\left(D_{1}, D_{2}\right) \in\left(0, \sigma^{2}\right] \times\left(0, \sigma^{2}\right] \backslash \mathscr{D}_{1}$

$$
R_{S_{1}, S_{2}}\left(D_{1}, D_{2}\right)=\frac{1}{2} \log _{2}\left(\frac{\operatorname{det} \mathrm{K}_{S S}}{\operatorname{det}\left(\mathrm{K}_{S S}-\mathrm{K}_{\hat{S} \hat{S}}\right)}\right),
$$

where $\mathrm{K}_{\hat{S} \hat{S}}$ is the covariance matrix of the random vector (43) corresponding to the above $R^{*}$ and $c^{*}$.

Proof. See Section A.3.

Based on Theorem A.1, we now evaluate $R_{S_{1}, S_{2}}\left(D_{1}, D_{2}\right)$ for all $\left(D_{1}, D_{2}\right) \in\left(0, \sigma^{2}\right] \times$ $\left(0, \sigma^{2}\right] \backslash \mathscr{D}_{1}$ by expressing $\mathrm{K}_{\hat{S} \hat{S}}$ in terms of $\left(D_{1}, D_{2}\right)$. This is done in the following lemma.

Lemma A.1. For $\left(D_{1}, D_{2}\right) \in\left(0, \sigma^{2}\right] \times\left(0, \sigma^{2}\right] \backslash \mathscr{D}_{1}$, the covariance matrix $\mathrm{K}_{\hat{S} \hat{S}}$ in (45) is given by

$$
\mathrm{K}_{\hat{S} \hat{S}}=\left\{\begin{array}{cc}
\left(\begin{array}{cc}
\sigma^{2}-D_{1} & \rho \sigma^{2} \\
\rho \sigma^{2} & \sigma^{2}-D_{2}
\end{array}\right) & \text { if }\left(D_{1}, D_{2}\right) \in \mathscr{D}_{2} \\
\left(\begin{array}{cc}
\sigma^{2}-D_{1} & \sqrt{\left(\sigma^{2}-D_{1}\right)\left(\sigma^{2}-D_{2}\right)} \\
\sqrt{\left(\sigma^{2}-D_{1}\right)\left(\sigma^{2}-D_{2}\right)} & \sigma^{2}-D_{2}
\end{array}\right) & \text { if }\left(D_{1}, D_{2}\right) \in \mathscr{D}_{3} .
\end{array}\right.
$$


Proof. See Section A.4

Combining Lemma A.1 with Theorem A.1 gives

$$
R_{S_{1}, S_{2}}\left(D_{1}, D_{2}\right)= \begin{cases}\frac{1}{2} \log _{2}^{+}\left(\frac{\sigma^{4}\left(1-\rho^{2}\right)}{D_{1} D_{2}}\right) & \text { if }\left(D_{1}, D_{2}\right) \in \mathscr{D}_{2} \\ \frac{1}{2} \log _{2}^{+}\left(\frac{\sigma^{4}\left(1-\rho^{2}\right)}{D_{1} D_{2}-\left(\rho \sigma^{2}-\sqrt{\left(\sigma^{2}-D_{1}\right)\left(\sigma^{2}-D_{2}\right)}\right)^{2}}\right) & \text { if }\left(D_{1}, D_{2}\right) \in \mathscr{D}_{3} .\end{cases}
$$

The complete expression of $R_{S_{1}, S_{2}}\left(D_{1}, D_{2}\right)$ for all $\left(D_{1}, D_{2}\right) \in\left(0, \sigma^{2}\right] \times\left(0, \sigma^{2}\right]$ is now obtained by combining (47) with (41).

To conclude this section, we briefly point out that the above described algorithm easily extends to multi-variate Gaussians with more than two components.

Remark A.2. The extension of the above algorithm to multivariate Gaussians with more than two components is straight forward. For a source with $\nu$ components $\left(S_{1}, S_{2}, \ldots, S_{\nu}\right)$, the scaling needs to be applied to $\nu-1$ components, e.g. each of the components $S_{2}, \ldots, S_{\nu}$ is scaled with a respective coefficient $c_{2}, \ldots c_{\nu}$ in $\mathbb{R}^{+}$. The tuple $\left(S_{1}, c_{2} S_{2}, \ldots, c_{\nu} S_{\nu}\right)$ is then unitarily decorrelated to the tuple $\left(V_{1}, V_{2}, \ldots, V_{\nu}\right)$ on which reverse waterfilling is applied. The reconstructions $\left(\hat{S}_{1}, \hat{S}_{2}, \ldots, \hat{S}_{\nu}\right)$ then follow from $\left(\hat{V}_{1}, \hat{V}_{2}, \ldots, \hat{V}_{\nu}\right)$ by the reverse of the transformation that mapped $\left(S_{1}, S_{2}, \ldots, S_{\nu}\right)$ to $\left(V_{1}, V_{2}, \ldots, V_{\nu}\right)$. The corresponding extension of Theorem A.1 follows easily from the proof in Section A.3.

\section{A.3 Proof of Theorem A.1}

To prove Theorem $\mathrm{A} .1$ we show that for every distortion pair $\left(D_{1}, D_{2}\right) \in\left(0, \sigma^{2}\right] \times$ $\left(0, \sigma^{2}\right] \backslash \mathscr{D}_{1}$ there exists a rate $R^{*} \in \mathbb{R}^{+}$and a scaling coefficient $c^{*} \in \mathbb{R}^{+}$such that

$\mathrm{E}\left[\left(S_{1}-\hat{S}_{1}\right)^{2}\right]=D_{1}, \mathrm{E}\left[\left(S_{2}-\hat{S}_{2}\right)^{2}\right]=D_{2}$, and $R^{*}=R_{S_{1}, S_{2}}\left(D_{1}, D_{2}\right)$. The proof is then completed by computing $I\left(S_{1}, S_{2} ; \hat{S}_{1}, \hat{S}_{2}\right)$ and showing that it is equal to the RHS of (45).

We begin by introducing the distortion regions $\mathscr{D}(R)$ and $\mathscr{D}_{c}(R)$. The region $\mathscr{D}(R)$ is the set of all pairs $\left(D_{1}, D_{2}\right)$ that are achievable with rate $R$ for the source pair $\left(S_{1}, S_{2}\right)$. Similarly, the region $\mathscr{D}_{c}(R)$ is the set of all pairs $\left(D_{1}, D_{2}\right)$ that are achievable with rate $R$ on the scaled source pair $\left(S_{1}, c S_{2}\right)$. The following two remarks state some properties of these two regions.

Remark A.3. The regions $\mathscr{D}(R)$ and $\mathscr{D}_{c}(R)$ satisfy the following properties:

i) $\mathscr{D}(R)$ and $\mathscr{D}_{c}(R)$ are convex.

ii) $\mathscr{D}_{c}(R)$ is a linearly scaled version of $\mathscr{D}(R)$. The scaling is in the dimension of the $D_{2}$-axis and of factor $c^{2}$.

iii) For a source satisfying $\operatorname{Var}\left(S_{1}\right)=\operatorname{Var}\left(S_{2}\right)$, the region $\mathscr{D}(R)$ is symmetric with respect to the line $D_{1}=D_{2}$.

Proof of Remark A.3. Part i) follows by a time-sharing argument. Part ii) follows by showing that if a distortion pair $\left(D_{1}, D_{2}\right)$ is achievable with rate $R$ on $\left(S_{1}, S_{2}\right)$, then also 
$\left(D_{1}, c^{2} D_{2}\right)$ is achievable with rate $R$ on $\left(S_{1}, c S_{2}\right)$, and vice versa. This follows since if a reconstruction pair $\left(\hat{S}_{1}, \hat{S}_{2}\right)$ results in distortions $\left(D_{1}, D_{2}\right)$ on $\left(S_{1}, S_{2}\right)$, then the scaled reconstructions $\left(\hat{S}_{1}, c \hat{S}_{2}\right)$ result in distortions $\left(D_{1}, c^{2} D_{2}\right)$ on $\left(S_{1}, c S_{2}\right)$. Part iii) follows since for $\left(S_{1}, S_{2}\right)$ jointly Gaussian with same variances, the distribution between $S_{1}$ and $S_{2}$ is perfectly symmetric. Hence, if with rate $R$ the pair $\left(D_{1}, D_{2}\right)=(a, b)$ is achievable, then also the pair $\left(D_{1}, D_{2}\right)=(b, a)$ is achievable.

Remark A.4. The scaled reconstruction pair $\left(\hat{S}_{1}, c \hat{S}_{2}\right)$, where $\left(\hat{S}_{1}, \hat{S}_{2}\right)$ is the result from our algorithm at rate $R$, yields the expected distortion pair $\left(D_{1}, D_{2}\right)$ of minimal sum $D_{1}+D_{2}$ in $\mathscr{D}_{c}(R)$.

Proof of Remark $A$.4. We denote by $\Delta_{1}$ and $\Delta_{2}$ the distortion on $V_{1}$ and $V_{2}$, i.e.

$$
\Delta_{i}=\mathrm{E}\left[\left(V_{i}-\hat{V}_{i}\right)^{2}\right] \quad i \in\{1,2\} .
$$

By definition of the reverse waterfilling solution, the reconstruction pair $\left(\hat{V}_{1}, \hat{V}_{2}\right)$ achieves the distortion pair $\left(\Delta_{1}, \Delta_{2}\right)$ on $\left(V_{1}, V_{2}\right)$ of minimal sum $\Delta_{1}+\Delta_{2}$ among all rate- $R$ achievable pairs $\left(\Delta_{1}, \Delta_{2}\right)$. Since $\left(S_{1}, c S_{2}\right)$ relates to $\left(V_{1}, V_{2}\right)$ by the same unitary transformation that relates $\left(\hat{S}_{1}, c \hat{S}_{2}\right)$ to $\left(\hat{V}_{1}, \hat{V}_{1}\right)$, the sum of the distortions $D_{1}+D_{2}$ on $\left(S_{1}, c S_{2}\right)$ equals $\Delta_{1}+\Delta_{2}$. Hence, if $\left(\Delta_{1}, \Delta_{2}\right)$ is the pair of minimal sum among all rate- $R$ achievable pairs on $\left(V_{1}, V_{2}\right)$, then $D_{1}, D_{2}$ is the pair of minimal sum among all rate- $R$ achievable pairs on $\left(S_{1}, c S_{2}\right)$, i.e., in $\mathscr{D}_{c}(R)$.

We are now ready to start with the proof of Theorem A.1.

Proof of Theorem A.1. We first prove the Parts i) and ii) together. To this end, we begin by arguing that for every boundary point $\left(D_{1}, D_{2}\right)$ of $\mathscr{D}(R)$ that falls out of $\mathscr{D}_{1}$ and satisfies $D_{1} \leq D_{2}$, there exists some $c \in(0,1]$ such that our algorithm yields $\left(D_{1}, D_{2}\right)$. To begin, consider $c=1$. For this case, the distortion pair resulting from our scheme is the boundary point of $\mathscr{D}(R)$ that satisfies $D_{1}=D_{2}$. This can be seen by noticing that for $c=1$ the regions $\mathscr{D}(R)$ and $\mathscr{D}_{c}(R)$ coincide. Thus, by Remark A.4, the distortion pair $\left(D_{1}, D_{2}\right)$ resulting on $\left(S_{1}, S_{2}\right)$ is the one of smallest sum $D_{1}+D_{2}$ in $\mathscr{D}(R)$. This distortion pair is the point on the boundary of $\mathscr{D}(R)$ that satisfies $D_{1}=D_{2}$, since, by Remark A.3, the region $\mathscr{D}(R)$ is convex and symmetric with respect to the line satisfying $D_{1}=D_{2}$.

Next, consider $0<c<1$. The key idea of our algorithm is illustrated in Figure 8. Subplot a) shows the distortion region $\mathscr{D}_{c}(R)$, and Subplot b) shows the distortion region $\mathscr{D}(R)$. The distortion pair $\left(D_{1}^{*}, D_{2}^{*}\right)$ resulting from our algorithm on $\left(S_{1}, S_{2}\right)$ and the corresponding distortion pair $\left(D_{1}^{*}, c^{2} D_{2}^{*}\right)$ on $\left(S_{1}, c S_{2}\right)$ are marked with a dot in Subplot b) and Subplot a) respectively. By Remark A.4 the distortion pair $\left(D_{1}^{*}, c^{2} D_{2}^{*}\right)$ is the point of smallest sum in $\mathscr{D}_{c}(R)$. Hence, graphically this point is characterized as the point on the boundary of $\mathscr{D}_{c}(R)$ for which the straight line of slope -1 containing it has the smallest ordinat 11 . By this graphical characterization of $\left(D_{1}^{*}, c^{2} D_{2}^{*}\right)$ it can now be seen that the smaller $c$ gets, i.e., the more $\mathscr{D}_{c}(R)$ is shrunk along the $D_{2}$-axis,

\footnotetext{
${ }^{1}$ This characterization follows since for any $\xi \in \mathbb{R}$ the set of pairs $\left(D_{1}, D_{2}\right)$ with sum $D_{1}+D_{2}=\xi$ is the straight line of slope -1 and ordinate $\xi$. Hence, if the ordinate $\xi$ of the straight line is minimized then the sum $D_{1}+D_{2}$ is minimized.
} 


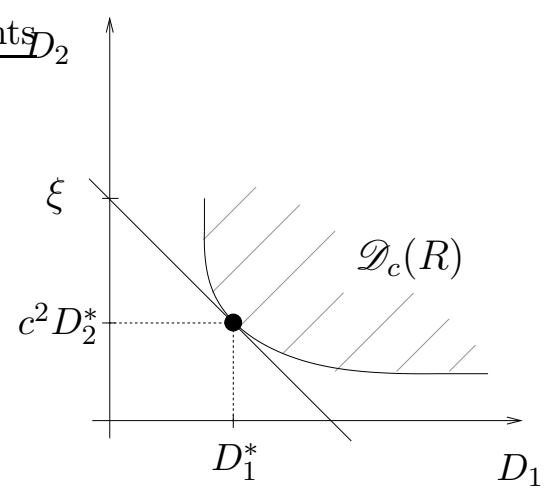

a)

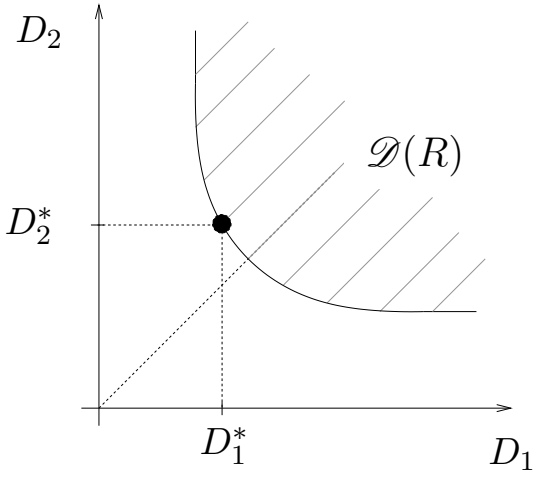

b)

Figure 8: Source scaling combined with reverse waterfilling.

the smaller the associated distortion $D_{1}^{*}$ gets. And as $c \rightarrow 0$, the distortion $D_{1}^{*}$ tends to the minimal value of $\sigma^{2} 2^{-2 R}$. Thus, by the linear relationship between $\mathscr{D}_{c}(R)$ and $\mathscr{D}(R)$, and by the convexity of $\mathscr{D}(R)$, it follows that for $c \in(0,1]$ our algorithm can achieve any $\left(D_{1}, D_{2}\right)$ on the boundary of $\mathscr{D}(R)$ for which $\sigma^{2} 2^{-2 R}<D_{1} \leq D_{2}$.

For $c>1$, it can be shown by similar arguments that our algorithm can achieve any $\left(D_{1}, D_{2}\right)$ on the boundary of $\mathscr{D}(R)$ for which $\sigma^{2} 2^{-2 R}<D_{2}<D_{1}$, i.e., for which $D_{2}<D_{1}<\sigma^{2}\left(1-\rho^{2}\right)+\rho^{2} D_{1}$. Hence, for $c \in \mathbb{R}^{+}$our algorithm can achieve any boundary point of $\mathscr{D}(R)$ in $\left(0, \sigma^{2}\right] \times\left(0, \sigma^{2}\right] \backslash \mathscr{D}_{1}$.

To complete the proof of Parts i) and ii), we now show that with the appropriate $R$, the boundary of $\mathscr{D}(R)$ can indeed cover any point in $\left[0, \sigma^{2}\right] \times\left[0, \sigma^{2}\right] \backslash \mathscr{D}_{1}$, and that for each such boundary point of $\mathscr{D}(R)$ the rate-distortion function satisfies $R_{S_{1}, S_{2}}\left(D_{1}, D_{2}\right)=R$.

Lemma A.2. For every distortion pair $\left(D_{1}, D_{2}\right) \in\left(0, \sigma^{2}\right] \times\left(0, \sigma^{2}\right] \backslash \mathscr{D}_{1}$, there exists some rate $R^{*} \in \mathbb{R}^{+}$such that $\left(D_{1}, D_{2}\right)$ is in the boundary of $\mathscr{D}\left(R^{*}\right)$. Furthermore, for each such boundary point

$$
R_{S_{1}, S_{2}}\left(D_{1}, D_{2}\right)=R^{*} .
$$

Proof of Lemma A.2. We know that each distortion pair $\left(D_{1}, D_{2}\right)$ resulting from our algorithm at rate $R$ lies on the boundary of $\mathscr{D}(R)$, and, by (42) and (43), is given by

$$
D_{1}^{*}=a_{1}^{2} \Delta_{1}+a_{2}^{2} \Delta_{2}, \quad D_{2}^{*}=\frac{a_{2}^{2}}{c^{2}} \Delta_{1}+\frac{a_{1}^{2}}{c^{2}} \Delta_{2} .
$$

Since the distortion pair $\left(\Delta_{1}, \Delta_{2}\right)$ results from reverse waterfilling, either both of the distortions are strictly and continuously decreasing in $R$, or one of them is constant while the other is strictly and continuously decreasing in $R$. Thus, since for every $c \in \mathbb{R}^{+}$, the coefficients $a_{1}$ and $a_{2}$ are both non-zero and constant, both distortions $D_{1}^{*}$ and $D_{2}^{*}$ are strictly and continuously decreasing in $R$. Hence, for every $\epsilon>0$, no boundary point $\left(D_{1}, D_{2}\right)$ of $\mathscr{D}(R)$ belongs to $\mathscr{D}(R-\epsilon)$, and therefore, every boundary point $\left(D_{1}, D_{2}\right)$ of $\mathscr{D}(R)$ satisfies

$$
R_{S_{1}, S_{2}}\left(D_{1}, D_{2}\right)=R
$$

The proof of of Lemma A.2 is now completed by recalling that for each $R \in \mathbb{R}^{+}$the region $\mathscr{D}(R)$ is convex, and that for every fixed $c \in \mathbb{R}^{+}$the corresponding boundary 
point in $\mathscr{D}(R)$ is evolving continuously in $R$. Thus, since for $R=0$ the distortion region is given by $\mathscr{D}(R)=\left(\sigma^{2}, \sigma^{2}\right)$ and for $R \rightarrow \infty$ the distortion region is given by $\mathscr{D}(R)=\left[0, \sigma^{2}\right] \times\left[0, \sigma^{2}\right]$, it follows that with the appropriate $R \in \mathbb{R}^{+}$the boundary of $\mathscr{D}(R)$ can cover any point in $\left[0, \sigma^{2}\right] \times\left[0, \sigma^{2}\right]$.

We now turn to the proof of Part iii). The proof consists of computing $I\left(S_{1}, S_{2} ; \hat{S}_{1}, \hat{S}_{2}\right)$ and showing that it is equal to the RHS of (45). We use the shorthand notation $\tilde{\mathbf{V}}$ for $\left(V_{1}, V_{2}, \hat{V}_{1}, \hat{V}_{2}\right)$ and $\tilde{\mathbf{S}}$ for $\left(S_{1}, S_{2}, \hat{S}_{1}, \hat{S}_{2}\right)$. Thus,

$$
\tilde{\mathbf{S}}=\left(\begin{array}{cc}
B & 0 \\
0 & B
\end{array}\right) \tilde{\mathbf{V}}
$$

Since $\tilde{\mathbf{S}}$ and $\tilde{\mathbf{V}}$ are related by a linear transformation and since $\tilde{\mathbf{V}}$ is a zero-mean Gaussian vector, it follows that also $\tilde{\mathbf{S}}$ is a zero-mean Gaussian vector. Thus,

$$
I\left(S_{1}, S_{2} ; \hat{S}_{1}, \hat{S}_{2}\right)=\frac{1}{2} \log _{2}\left(\frac{\operatorname{det} \mathrm{K}_{S S} \operatorname{det} \mathrm{K}_{\hat{S} \hat{S}}}{\operatorname{det} \mathrm{K}_{\tilde{S} \tilde{S}}}\right),
$$

where $\mathrm{K}_{\tilde{S} \tilde{S}}$ is the covariance matrix of $\tilde{\mathbf{S}}$. The determinant $\operatorname{det} \mathrm{K}_{\tilde{S} \tilde{S}}$ in (50) is now expressed in terms of $\mathrm{K}_{S S}$ and $\mathrm{K}_{\hat{S} \hat{S}}$. Since the reconstruction pair $\left(\hat{S}_{1}, \hat{S}_{2}\right)$ achieves a boundary point of $\mathscr{D}(R)$, it must satisfy the orthogonality principle and thus $\mathrm{K}_{S \hat{S}}=\mathrm{K}_{\hat{S} \hat{S}}$, where $\mathrm{K}_{S \hat{S}}$ denoted the cross-covariance matrix between $\left(S_{1}, S_{2}\right)$ and $\left(\hat{S}_{1}, \hat{S}_{2}\right)$. Hence,

$$
\mathrm{K}_{\tilde{S} \tilde{S}}=\left(\begin{array}{cc}
\mathrm{K}_{S S} & \mathrm{~K}_{\hat{S} \hat{S}} \\
\mathrm{~K}_{\hat{S} \hat{S}} & \mathrm{~K}_{\hat{S} \hat{S}}
\end{array}\right)
$$

Applying Schur's complement [22, pp. 21] to (51) gives

$$
\operatorname{det} \mathrm{K}_{\tilde{S} \tilde{S}}=\operatorname{det} \mathrm{K}_{\hat{S} \hat{S}} \cdot \operatorname{det}\left(\mathrm{K}_{S S}-\mathrm{K}_{\hat{S} \hat{S}}\right) \text {. }
$$

Combining (52) with (50), and using that the law of the $\left(S_{1}, S_{2}, \hat{S}_{1}, \hat{S}_{2}\right)$ at hand is a solution to the optimization problem in (40), gives

$$
R_{S_{1}, S_{2}}\left(D_{1}, D_{2}\right)=\frac{1}{2} \log _{2}\left(\frac{\operatorname{det} \mathrm{K}_{S S}}{\operatorname{det}\left(\mathrm{K}_{S S}-\mathrm{K}_{\hat{S} \hat{S}}\right)}\right),
$$

for all $\left(D_{1}, D_{2}\right) \in\left(0, \sigma^{2}\right] \times\left(0, \sigma^{2}\right] \backslash \mathscr{D}_{1}$.

\section{A.4 Proof of Lemma A.1}

Lemma A.1 expresses $\mathrm{K}_{\hat{S} \hat{S}}$ in terms of $\left(D_{1}, D_{2}\right)$. To obtain the stated expression, we first use that $\mathrm{K}_{\hat{S} \hat{S}}=\mathrm{BK}_{\hat{V} \hat{V}} \mathrm{~B}^{\top}$ and begin by writing $\mathrm{K}_{\hat{V} \hat{V}}$ in terms of the variances of $V_{1}$ and $V_{2}$, and in terms of the distortions on those two components. To this end, we denote by $\lambda_{1}$ and $\lambda_{2}$ the variances of $V_{1}$ and $V_{2}$, i.e.

$$
\lambda_{i}=\operatorname{Var}\left(V_{i}\right) \quad i \in\{1,2\} .
$$


By (42), the variances $\lambda_{1}$ and $\lambda_{2}$ are the eigenvalues of the covariance matrix of $\left(S_{1}, c S_{2}\right)$, which are given by

$$
\begin{aligned}
& \lambda_{1}=\frac{\sigma^{2}}{2}\left(1+c^{2}-\sqrt{1-2 c^{2}\left(1-2 \rho^{2}\right)+c^{4}}\right), \\
& \lambda_{2}=\frac{\sigma^{2}}{2}\left(1+c^{2}+\sqrt{1-2 c^{2}\left(1-2 \rho^{2}\right)+c^{4}}\right) .
\end{aligned}
$$

The covariance matrix $\mathrm{K}_{\hat{V} \hat{V}}$ can now easily be expressed in terms of $\lambda_{1}, \lambda_{2}, \Delta_{1}$, and $\Delta_{2}$. Since for every $c \in \mathbb{R}^{+}$the expressions in (54) yield $\lambda_{1} \leq \lambda_{2}$, the reverse waterfilling on $\left(V_{1}, V_{2}\right)$ satisfies

$$
\Delta_{1}= \begin{cases}\Delta_{2} & \text { if } 0 \leq \Delta_{2} \leq \lambda_{1} \\ \lambda_{1} & \text { if } \lambda_{1}<\Delta_{2} \leq \lambda_{2} .\end{cases}
$$

The corresponding covariance matrix $\mathrm{K}_{\hat{V} \hat{V}}$ is thus

$$
\mathrm{K}_{\hat{V} \hat{V}}= \begin{cases}\left(\begin{array}{cc}
\lambda_{1}-\Delta_{2} & 0 \\
0 & \lambda_{2}-\Delta_{2}
\end{array}\right) & \text { if } 0 \leq \Delta_{2} \leq \lambda_{1} \\
\left(\begin{array}{cc}
0 & 0 \\
0 & \lambda_{2}-\Delta_{2}
\end{array}\right) & \text { if } \lambda_{1}<\Delta_{2} \leq \lambda_{2} .\end{cases}
$$

Based on (56), we now express $\mathrm{K}_{\hat{S} \hat{S}}=\mathrm{BK}_{\hat{V} \hat{V}} \mathrm{~B}^{\top}$ in terms of $D_{1}$ and $D_{2}$. To this end, we denote the set of all distortion pairs $\left(D_{1}, D_{2}\right)$ resulting from $0 \leq \Delta_{2}<\lambda_{1}$ by $\mathscr{A}$ and the set of all distortion pairs resulting from $\lambda_{1} \leq \Delta_{2} \leq \lambda_{2}$ by $\mathscr{B}$. The expressions for $\mathscr{A}$ and $\mathscr{B}$, and for $\mathrm{K}_{\hat{S} \hat{S}}$, in terms of $D_{1}$ and $D_{2}$, are now given in the following two lemmas.

Lemma A.3. For $\left(D_{1}, D_{2}\right) \in\left(0, \sigma^{2}\right] \times\left(0, \sigma^{2}\right] \backslash \mathscr{D}_{1}$, the covariance matrix $\mathrm{K}_{\hat{S} \hat{S}}$ in (45) is given by

$$
\mathrm{K}_{\hat{S} \hat{S}}=\left\{\begin{array}{cc}
\left(\begin{array}{cc}
\sigma^{2}-D_{1} & \rho \sigma^{2} \\
\rho \sigma^{2} & \sigma^{2}-D_{2}
\end{array}\right) & \text { if }\left(D_{1}, D_{2}\right) \in \mathscr{A} \\
\left(\begin{array}{cc}
\sigma^{2}-D_{1} & \sqrt{\left(\sigma^{2}-D_{1}\right)\left(\sigma^{2}-D_{2}\right)} \\
\sqrt{\left(\sigma^{2}-D_{1}\right)\left(\sigma^{2}-D_{2}\right)} & \sigma^{2}-D_{2}
\end{array}\right) & \text { if }\left(D_{1}, D_{2}\right) \in \mathscr{B} .
\end{array}\right.
$$

Proof. See Section A.4.2.

Lemma A.4. The regions $\mathscr{A}$ and $\mathscr{B}$ are given by

$$
\mathscr{A}=\mathscr{D}_{2} \quad \text { and } \quad \mathscr{B}=\mathscr{D}_{3} .
$$

Proof. See Section A.4.1.

Combining Lemma A.3 and Lemma A.4 yields Lemma A.1. 
Proposition A.1. Every $\left(D_{1}, D_{2}\right)$ in $\mathscr{D}_{1} \cup \mathscr{D}_{3}$ can be achieved with rate $R_{S_{1}, S_{2}}\left(D_{1}, D_{2}\right)$ by optimally describing a linear combination of the sequences $\mathbf{S}_{1}$ and $\mathbf{S}_{2}$.

Proof. We prove Proposition A.1 for the regions $\mathscr{D}_{1}$ and $\mathscr{D}_{3}$ separately. For $\mathscr{D}_{1}$ the proof follows immediately from Section A.1 where it is shown that every $\left(D_{1}, D_{2}\right)$ in $\mathscr{D}_{1}$ is achieved with rate $R_{S_{1}, S_{2}}\left(D_{1}, D_{2}\right)$ either by optimally describing $\mathbf{S}_{1}$ or by optimally describing $\mathbf{S}_{2}$. For $\mathscr{D}_{3}$ the proof follows from combining (56) with Lemma A.4, from which it follows that every $\left(D_{1}, D_{2}\right)$ in $\mathscr{D}_{3}$ is achieved with rate $R_{S_{1}, S_{2}}\left(D_{1}, D_{2}\right)$ by optimally describing the sequence of corresponding $V_{2}$-components of $\left(\mathbf{S}_{1}, \mathbf{S}_{2}\right)$. This sequence, by the definition of $V_{2}$, is a linear combination of $\mathbf{S}_{1}$ and $\mathbf{S}_{2}$.

It remains to prove Lemma A.3 and Lemma A.4. We begin with Lemma A.4

\section{A.4.1 Proof of Lemma A.4}

Lemma A.4 determines the sets $\mathscr{A}$ and $\mathscr{B}$ in terms of the distortions $D_{1}$ and $D_{2}$. To prove this lemma we first derive the expression for $\mathscr{A}$. The expression for $\mathscr{B}$ will then be deduced by noticing that $\mathscr{A}$ and $\mathscr{B}$ form a partition of $\left(0, \sigma^{2}\right] \times\left(0, \sigma^{2}\right] \backslash \mathscr{D}_{1}$.

The region $\mathscr{A}$ is defined as the set of all $\left(D_{1}, D_{2}\right)$ deriving from $0 \leq \Delta_{2} \leq \lambda_{1}$. To translate this condition to an expression in terms of $D_{1}$ and $D_{2}$, we express $\Delta_{2}$ in terms of $D_{1}$ and $D_{2}$.

For $0 \leq \Delta_{2} \leq \lambda_{1}$ however, the reverse waterfilling solution in (55) yields $\Delta_{1}=\Delta_{2}$ such that (48) simplifies to

$$
D_{1}=\Delta_{2} \quad D_{2}=\frac{1}{c^{2}} \Delta_{2}
$$

Thus, the region $\mathscr{A}$ can be restated as the set of all pairs $\left(D_{1}, D_{2}\right) \in\left(0, \sigma^{2}\right] \times\left(0, \sigma^{2}\right]$ satisfying $0 \leq D_{1} \leq \lambda_{1}$ and $D_{1} / D_{2}=c^{2}$. Writing out $\lambda_{1}$ as in (54) and substituting therein $c^{2}$ by $D_{1} / D_{2}$ yields

$$
0 \leq D_{2}<\left(\sigma^{2}\left(1-\rho^{2}\right)-D_{1}\right) \frac{\sigma^{2}}{\sigma^{2}-D_{1}} .
$$

On the other hand, substituting the RHS of (54) in $D_{1}=\lambda_{1}$ leads to

$$
\max _{\left(D_{1}, D_{2}\right) \in \mathscr{D}_{2}} D_{1}=\sigma^{2}\left(1-\rho^{2}\right)
$$

where the maximum is obtained as $c \rightarrow \infty$. Thus, the region $\mathscr{A}$ can finally be restated as

$$
\mathscr{A}=\left\{\left(D_{1}, D_{2}\right): 0 \leq D_{1} \leq \sigma^{2}\left(1-\rho^{2}\right), 0 \leq D_{2}<\left(\sigma^{2}\left(1-\rho^{2}\right)-D_{1}\right) \frac{\sigma^{2}}{\sigma^{2}-D_{1}}\right\},
$$

i.e., $\mathscr{A}=\mathscr{D}_{2}$.

We now turn to the evaluation of $\mathscr{B}$. As can be verified by help of (48) the two sets $\mathscr{A}$ and $\mathscr{B}$ form a partition of $\left(0, \sigma^{2}\right] \times\left(0, \sigma^{2}\right] \backslash \mathscr{D}_{1}$. Thus, the region $\mathscr{B}$ is given by 


$$
\begin{aligned}
& \left(0, \sigma^{2}\right] \times\left(0, \sigma^{2}\right] \backslash\left(\mathscr{A} \cup \mathscr{D}_{1}\right) \text {. Hence, } \\
& \mathscr{B}=\left\{\left(D_{1}, D_{2}\right): 0 \leq D_{1} \leq \sigma^{2}\left(1-\rho^{2}\right),\right. \\
& \left(\sigma^{2}\left(1-\rho^{2}\right)-D_{1}\right) \frac{\sigma^{2}}{\sigma^{2}-D_{1}} \leq D_{2}<\sigma^{2}\left(1-\rho^{2}\right)+\rho^{2} D_{1} ; \\
& \\
& \sigma^{2}\left(1-\rho^{2}\right)<D_{1} \leq \sigma^{2}, \\
& \left.\frac{D_{1}-\sigma^{2}\left(1-\rho^{2}\right)}{\rho^{2}}<D_{2}<\sigma^{2}\left(1-\rho^{2}\right)+\rho^{2} D_{1}\right\},
\end{aligned}
$$

i.e., $\mathscr{B}=\mathscr{D}_{3}$.

\section{A.4.2 Proof of Lemma A.3}

Lemma A.3 gives an explicit expression of $\mathrm{K}_{\hat{S} \hat{S}}$ for $\left(D_{1}, D_{2}\right) \in \mathscr{A}$ and for $\left(D_{1}, D_{2}\right) \in \mathscr{B}$. The derivations are based on the expression for $\mathrm{K}_{\hat{V} \hat{V}}$ in (56). Combining (56) with $\mathrm{K}_{\hat{S} \hat{S}}=\mathrm{BK}_{\hat{V} \hat{V}} \mathrm{~B}^{\top}$ gives

$$
\mathrm{K}_{\hat{S} \hat{S}}=\left\{\begin{array}{cc}
\left(\begin{array}{cc}
a_{1}^{2} \lambda_{1}+a_{2}^{2} \lambda_{2}-\Delta_{2} & \frac{a_{1} a_{2}}{c}\left(\lambda_{2}-\lambda_{1}\right) \\
\frac{a_{1} a_{2}}{c}\left(\lambda_{2}-\lambda_{1}\right) & \frac{1}{c^{2}}\left(a_{2}^{2} \lambda_{1}+a_{1}^{2} \lambda_{2}-\Delta_{2}\right)
\end{array}\right) & \text { if }\left(D_{1}, D_{2}\right) \in \mathscr{A} \\
\left(\begin{array}{cc}
a_{2}^{2}\left(\lambda_{2}-\Delta_{2}\right) & \frac{a_{1} a_{2}}{c}\left(\lambda_{2}-\Delta_{2}\right) \\
\frac{a_{1} a_{2}}{c}\left(\lambda_{2}-\Delta_{2}\right) & \frac{a_{1}^{2}}{c^{2}}\left(\lambda_{2}-\Delta_{2}\right)
\end{array}\right) & \text { if }\left(D_{1}, D_{2}\right) \in \mathscr{B} .
\end{array}\right.
$$

For $\left(D_{1}, D_{2}\right) \in \mathscr{A}$, we now express the variables in (60) in terms of $D_{1}$ and $D_{2}$. From (58) it follows that

$$
\Delta_{2}=D_{1}, \quad \text { and } \quad c^{2}=\frac{D_{1}}{D_{2}}
$$

Furthermore, the coefficients $a_{1}$ and $a_{2}$ are determined by $a_{1}^{2}+a_{2}^{2}=1$ and

$$
a_{1}^{2}=\frac{2 c^{2} \rho^{2}}{1-2 c^{2}\left(1-2 \rho^{2}\right)+c^{4}+\left(1-c^{2}\right) \sqrt{1-2 c^{2}\left(1-2 \rho^{2}\right)+c^{4}}} .
$$

Combining (61), (62) and the expressions for $\lambda_{1}$ and $\lambda_{2}$ in (54) with (60), yields

$$
\mathrm{K}_{\hat{S} \hat{S}}=\left(\begin{array}{cc}
\sigma^{2}-D_{1} & \rho \sigma^{2} \\
\rho \sigma^{2} & \sigma^{2}-D_{2}
\end{array}\right) \quad \text { if }\left(D_{1}, D_{2}\right) \in \mathscr{A} .
$$

We now turn to the evaluation of $\mathrm{K}_{\hat{S} \hat{S}}$ for the region $\mathscr{B}$. For this region, no calculations are needed. It suffices to notice that since every optimal reconstruction pair $\left(\hat{S}_{1}, \hat{S}_{2}\right)$ satisfies the orthogonality principle, the main diagonal terms of the covariance matrix $\mathrm{K}_{\hat{S} \hat{S}}$ are

$$
a_{2}^{2}\left(\lambda_{2}-\Delta_{2}\right)=\sigma^{2}-D_{1} \quad \text { and } \quad \frac{a_{1}^{2}}{c^{2}}\left(\lambda_{2}-\Delta_{2}\right)=\sigma^{2}-D_{2},
$$


and that the anti-diagonal terms are both equal to the square-root of the product of the two main diagonal terms and thus are

$$
\frac{a_{1} a_{2}}{c}\left(\lambda_{2}-\Delta_{2}\right)=\sqrt{\left(\sigma^{2}-D_{1}\right)\left(\sigma^{2}-D_{2}\right)} .
$$

The covariance matrix $\mathrm{K}_{\hat{S} \hat{S}}$ for $\mathscr{B}$ is therefore

$$
\mathrm{K}_{\hat{S} \hat{S}}=\left(\begin{array}{cc}
\sigma^{2}-D_{1} & \sqrt{\left(\sigma^{2}-D_{1}\right)\left(\sigma^{2}-D_{2}\right)} \\
\sqrt{\left(\sigma^{2}-D_{1}\right)\left(\sigma^{2}-D_{2}\right)} & \sigma^{2}-D_{2}
\end{array}\right) \quad \text { if }\left(D_{1}, D_{2}\right) \in \mathscr{B} .
$$

\section{B Proof of Proposition 3.1}

Proposition 3.1 pertains to the point-to-point problem of Section 3, in which the source pair $\left\{\left(S_{1, k}, S_{2, k}\right)\right\}$ is to be transmitted over an AWGN channel. It states that for an achievable distortion pair $\left(D_{1}, D_{2}\right)$ for which the SNR of the channel satisfies $P / N \leq$ $\Gamma\left(D_{1}, \sigma^{2}, \rho\right)$, there exist $\alpha^{*}, \beta^{*} \geq 0$ such that

$$
\tilde{D}_{1}^{\mathrm{u}}\left(\alpha^{*}, \beta^{*}\right) \leq D_{1} \quad \text { and } \quad \tilde{D}_{2}^{\mathrm{u}}\left(\alpha^{*}, \beta^{*}\right) \leq D_{2} .
$$

The essence of Proposition 3.1 is that the uncoded scheme proposed in Section 3.3 achieves every distortion pair $\left(D_{1}, D_{2}\right)$ in $\mathscr{D}_{1} \cup \mathscr{D}_{3}$ with the least possible transmission power, i.e., with the $P$ for which

$$
R_{S_{1}, S_{2}}\left(D_{1}, D_{2}\right)=\frac{1}{2} \log _{2}\left(1+\frac{P}{N}\right) .
$$

In Proposition 3.1, the condition $\left(D_{1}, D_{2}\right) \in \mathscr{D}_{1} \cup \mathscr{D}_{3}$ is merely expressed in form of the threshold $\Gamma\left(D_{1}, \sigma^{2}, \rho\right)$ on $P / N$.

We start the proof by showing that the uncoded scheme indeed achieves every $\left(D_{1}, D_{2}\right) \in \mathscr{D}_{1} \cup \mathscr{D}_{3}$ with the least possible transmission power, respectively at the smallest $P / N$. To this end, let $\Psi\left(D_{1}, D_{2}\right)$ be the smallest $P / N$ at which $\left(D_{1}, D_{2}\right)$ is achievable, i.e.

$$
R_{S_{1}, S_{2}}\left(D_{1}, D_{2}\right)=\frac{1}{2} \log _{2}\left(1+\Psi\left(D_{1}, D_{2}\right)\right) .
$$

We now show that for every $\left(D_{1}, D_{2}\right) \in \mathscr{D}_{1} \cup \mathscr{D}_{3}$, there exist $\alpha^{*}, \beta^{*}$ such that the distortions resulting from the uncoded scheme at $P / N=\Psi\left(D_{1}, D_{2}\right)$ are $\left(\tilde{D}_{1}^{\mathrm{u}}(\alpha, \beta), \tilde{D}_{2}^{\mathrm{u}}(\alpha, \beta)\right)=$ $\left(D_{1}, D_{2}\right)$. To show this, we rely on Proposition A.1 (p. 31) and on the result of [1]. Proposition A.1 states for the corresponding source coding problem that if $\left(D_{1}, D_{2}\right)$ is in $\mathscr{D}_{1} \cup \mathscr{D}_{3}$ then $R_{S_{1}, S_{2}}\left(D_{1}, D_{2}\right)$ can be achieved by optimally describing a linear combination of the sequences $\mathbf{S}_{1}$ and $\mathbf{S}_{2}$. The result of [1] states that the minimum expected squared-error transmission of a Gaussian source over a AWGN channel is achieved by uncoded transmission. Thus, by combining Proposition A.1] with the result of [1] and

using that since $\left\{\left(S_{1, k}, S_{2, k}\right)\right\}$ are jointly Gaussian, each of their linear combination 
$\alpha \mathbf{S}_{1}+\beta \mathbf{S}_{2}$ is also Gaussian, it follows that every distortion pair $\left(D_{1}, D_{2}\right) \in \mathscr{D}_{1} \cup \mathscr{D}_{3}$ is achieved at $P / N=\Psi\left(D_{1}, D_{2}\right)$, by sending over the channel

$$
X_{k}^{\mathrm{u}}(\alpha, \beta)=\sqrt{\frac{P}{\sigma^{2}\left(\alpha^{2}+2 \rho \alpha \beta+\beta^{2}\right)}}\left(\alpha S_{1, k}+\beta S_{2, k}\right) \quad k \in\{1,2, \ldots, n\},
$$

with the appropriate $\alpha, \beta \geq 0$.

It remains to derive the threshold function $\Gamma$. To this end, first notice that for an arbitrary fixed $D_{1} \in\left[0, \sigma^{2}\right]$, the smaller the associated $D_{2}$ gets, the larger $\Psi\left(D_{1}, D_{2}\right)$ becomes, i.e., for a fixed $D_{1}$ the function $\Psi\left(D_{1}, D_{2}\right)$ is decreasing in $D_{2}$. Now, for every $D_{1} \in\left[0, \sigma^{2}\right]$, let $\bar{D}_{2}\left(D_{1}\right)$ be the smallest $D_{2}$ such that $\left(D_{1}, D_{2}\right) \in \mathscr{D}_{1} \cup \mathscr{D}_{3}$. Then, for every $D_{1} \in\left[0, \sigma^{2}\right]$

$$
\Gamma\left(D_{1}, \sigma^{2}, \rho\right)=\Psi\left(D_{1}, \bar{D}_{2}\left(D_{1}\right)\right) .
$$

Hence, it remains to evaluate $\Psi\left(D_{1}, \bar{D}_{2}\left(D_{1}\right)\right)$ for every $D_{1} \in\left[0, \sigma^{2}\right]$. We have

$$
\bar{D}_{2}\left(D_{1}\right)= \begin{cases}\left(\sigma^{2}\left(1-\rho^{2}\right)-D_{1}\right) \frac{\sigma^{2}}{\sigma^{2}-D_{1}} & \text { if } 0 \leq D_{1} \leq \sigma^{2}\left(1-\rho^{2}\right), \\ 0 & \text { if } D_{1}>\sigma^{2}\left(1-\rho^{2}\right) .\end{cases}
$$

For $D_{1}>\sigma^{2}\left(1-\rho^{2}\right)$ it immediately follows that $\Gamma\left(D_{1}, \sigma^{2}, \rho\right)=\infty$. For $0 \leq D_{1} \leq$ $\sigma^{2}\left(1-\rho^{2}\right)$ the value of $\Psi\left(D_{1}, \bar{D}_{2}\left(D_{1}\right)\right)$, and hence the value of $\Gamma\left(D_{1}, \sigma^{2}, \rho\right)$ follows from solving

$$
\frac{1}{2} \log _{2}^{+}\left(\frac{\sigma^{4}\left(1-\rho^{2}\right)}{D_{1} \bar{D}_{2}-\left(\rho \sigma^{2}-\sqrt{\left(\sigma^{2}-D_{1}\right)\left(\sigma^{2}-\bar{D}_{2}\right)}\right)^{2}}\right)=\frac{1}{2} \log _{2}\left(1+\frac{P}{N}\right)
$$

where we have used the shorthand notation $\bar{D}_{2}$ for $\bar{D}_{2}\left(D_{1}\right)$. From (65), we now get

$$
\rho \sigma^{2}-\sqrt{\left(\sigma^{2}-D_{1}\right)\left(\sigma^{2}-\bar{D}_{2}\right)}=0 .
$$

Thus, (66) reduces to

$$
\frac{\sigma^{4}\left(1-\rho^{2}\right)}{D_{1} \bar{D}_{2}}=1+\frac{P}{N}
$$

which, by (65), can be rewritten as

$$
\frac{P}{N}=\frac{\sigma^{4}\left(1-\rho^{2}\right)-2 \sigma^{2} D_{1}\left(1-\rho^{2}\right)+D_{1}^{2}}{D_{1}\left(\sigma^{2}\left(1-\rho^{2}\right)-D_{1}\right)} .
$$

This is the threshold given in Proposition 3.1 for $0 \leq D_{1} \leq \sigma^{2}\left(1-\rho^{2}\right)$.

To conclude the proof, we justify the restriction to $\alpha, \beta \geq 0$. This restriction is made because from the expressions for $\tilde{D}_{1}^{\mathrm{u}}(\alpha, \beta)$ and $\tilde{D}_{2}^{\mathrm{u}}(\alpha, \beta)$ it follows that it incurs no loss in performance. This is so, since $\rho \geq 0$, and thus the uncoded transmission scheme with the choice of $(\alpha, \beta)$ such that $\alpha \cdot \beta<0$ yields a distortion that is uniformly worse than the choice of $(|\alpha|,|\beta|)$, and every distortion pair achievable with $\alpha, \beta<0$, is also achievable with $(|\alpha|,|\beta|)$. Thus, without loss in performance, we can limit ourselves to $\alpha, \beta \geq 0$. 


\section{Proof of Theorem 4.1}

Theorem 4.1 applies to the multiple-access problem without feedback. For this problem it gives a necessary condition for the achievability of a distortion pair $\left(D_{1}, D_{2}\right)$. We begin with a reduction.

To state the proof in more detail, we make the following reduction.

Reduction C.1. There is no loss in optimality in restricting the encoding functions to satisfy

$$
\mathrm{E}\left[X_{i, k}\right]=0 \quad \text { for } i \in\{1,2\} \text {, and all } k \in \mathbb{Z} .
$$

Proof. We show that for every achievable tuple $\left(D_{1}, D_{2}, \sigma^{2}, \sigma^{2}, \rho, P_{1}, P_{2}, N\right)$, there exists a scheme with encoding functions satisfying (69) that achieves this tuple. To this end, let $\left(D_{1}, D_{2}, \sigma^{2}, \sigma^{2}, \rho, P_{1}, P_{2}, N\right)$ be an arbitrary achievable tuple. Further, let $\left\{f_{1}^{(n)}\right\},\left\{f_{2}^{(n)}\right\},\left\{\phi^{(n)}\right\}$ be sequences of encoding and decoding functions achieving this tuple. If the encoding functions $\left\{f_{1}^{(n)}\right\},\left\{f_{2}^{(n)}\right\}$ do not satisfy (69), then they can be adapted as follows. Before sending the codewords over the channel, the mean of the codewords is subtracted so as to satisfy (69). And at the channel output this subtraction is corrected by adding this term to the received sequence before decoding.

In view of Reduction C.1, we restrict ourselves, for the remainder of this proof to encoding functions that satisfy (69). The key element in the proof of Theorem 4.1 is the following.

Lemma C.1. Any scheme satisfying condition (69) and the original power constraints (4), also satisfies

$$
\frac{1}{n} \sum_{k=1}^{n} \mathrm{E}\left[\left(X_{1, k}+X_{2, k}\right)^{2}\right] \leq P_{1}+P_{2}+2 \rho \sqrt{P_{1} P_{2}} .
$$

Proof. See Appendix C.1.

Based on Lemma C.1, the proof of Theorem 4.1 is now obtained by relaxing the original problem as follows. First, the power constraint of (4) is replaced by the power constraint of (70). Then, under the power constraint of (170), the two transmitters are allowed to fully cooperate. These two relaxations reduce the original multiple-access problem to a point-to-point problem where the source sequence $\left\{\left(S_{1, k}, S_{2, k}\right)\right\}$ is to be transmitted over an AWGN channel of power constraint $P_{1}+P_{2}+2 \rho \sqrt{P_{1} P_{2}}$ and noise variance $N$. For this point-to-point problem, a necessary condition for the achievability of a distortion pair $\left(D_{1}, D_{2}\right)$ follows by source-channel separation, and is

$$
R_{S_{1}, S_{2}}\left(D_{1}, D_{2}\right) \leq \frac{1}{2} \log _{2}\left(1+\frac{P_{1}+P_{2}+2 \rho \sqrt{P_{1} P_{2}}}{N}\right) .
$$

It is now easy to conclude that (71) is also a necessary condition for the achievability of a distortion pair $\left(D_{1}, D_{2}\right)$ in the original multiple-access problem. This simply follows since (71) is a necessary condition for the achievability of a distortion pair $\left(D_{1}, D_{2}\right)$ in a relaxed version of the multiple-access problem. This concludes the proof of Theorem 4.1 , 


\section{C.1 Proof of Lemma C.1}

The key to Lemma C.1 is as follows:

Lemma C.2. For any coding scheme with encoding functions of the form (3) that satisfy the power constraints (44) and condition (69) of Reduction [C.1, and where the encoder input sequences are jointly Gaussian as in (11) with non-negative correlation coefficient $\rho$ and equal variances $\sigma_{1}^{2}=\sigma_{2}^{2}=\sigma^{2}$ (Reduction [2.1), any time- $k$ encoder outputs $X_{1, k}$ and $X_{2, k}$ satisfy

$$
\mathrm{E}\left[X_{1, k} X_{2, k}\right] \leq \rho \sqrt{\mathrm{E}\left[X_{1, k}^{2}\right]} \sqrt{\mathrm{E}\left[X_{2, k}^{2}\right]} .
$$

Proof. Lemma C.2 follows from two results from Maximum Correlation Theory. These results are stated now.

Theorem C.1 (Witsenhausen [21]). Consider a sequence of pairs of random variables $\left\{\left(W_{1, k}, W_{2, k}\right)\right\}$, where the pairs are independent (not necessarily identically distributed). Then,

$$
\sup _{g_{1}^{(n)}, g_{2}^{(n)}} \mathrm{E}\left[g_{1}^{(n)}\left(\mathbf{W}_{1}\right) g_{2}^{(n)}\left(\mathbf{W}_{2}\right)\right] \leq \sup _{\substack{1 \leq k \leq n \\ g_{1, k}, g_{2, k}}} \mathrm{E}\left[g_{1, k}\left(W_{1, k}\right) g_{2, k}\left(W_{2, k}\right)\right]
$$

where the supremum on the LHS of (73) is taken over all functions $g_{i}^{(n)}: \mathbb{R}^{n} \rightarrow \mathbb{R}$, satisfying

$$
\mathrm{E}\left[g_{i}^{(n)}\left(\mathbf{W}_{i}\right)\right]=0 \quad \mathrm{E}\left[\left(g_{i}^{(n)}\left(\mathbf{W}_{i}\right)\right)^{2}\right]=1 \quad i \in\{1,2\},
$$

and the supremum on the RHS of (73) is taken over all functions $g_{i, k}: \mathbb{R} \rightarrow \mathbb{R}$, satisfying

$$
\mathrm{E}\left[g_{i, k}^{(n)}\left(W_{i, k}\right)\right]=0 \quad \mathrm{E}\left[\left(g_{i, k}^{(n)}\left(W_{i, k}\right)\right)^{2}\right]=1 \quad i \in\{1,2\} .
$$

Proof. See [21, Theorem 1, p. 105].

Lemma C.3. Consider two jointly Gaussian random variables $W_{1, k}$ and $W_{2, k}$ with correlation coefficient $\rho_{k}$. Then,

$$
\sup _{g_{1, k}, g_{2, k}} \mathrm{E}\left[g_{1, k}\left(W_{1, k}\right) g_{2, k}\left(W_{2, k}\right)\right]=\left|\rho_{k}\right|,
$$

where the supremum is taken over all functions $g_{i, k}: \mathbb{R} \rightarrow \mathbb{R}$, satisfying

$$
\mathrm{E}\left[g_{i, k}\left(W_{i, k}\right)\right]=0 \quad \mathrm{E}\left[\left(g_{i, k}\left(W_{i, k}\right)\right)^{2}\right]=1 \quad i \in\{1,2\} .
$$

Proof. See [15, Lemma 10.2, p. 182]. 
Lemma C.2 is now merely a consequence of Theorem C.1 and Lemma C.3 applied to our setup. To see this, substitute $\mathbf{W}_{1}$ and $\mathbf{W}_{2}$ by the source sequences $\mathbf{S}_{1}$ and $\mathbf{S}_{2}$, and let the functions $g_{1}^{(n)}(\cdot)$ and $g_{2}^{(n)}(\cdot)$ be the encoding sub-functions that produce the time$k$ channel inputs $X_{1, k}$ and $X_{2, k}$, i.e., $g_{i}^{(n)}\left(\mathbf{S}_{i}\right)=X_{i, k}$. Then, for every $k \in\{1,2, \ldots, n\}$,

$$
\begin{aligned}
\frac{\mathrm{E}\left[X_{1, k} X_{2, k}\right]}{\sqrt{\mathrm{E}\left[X_{1, k}^{2}\right]} \sqrt{\mathrm{E}\left[X_{2, k}^{2}\right]}} & \leq \sup _{g_{1}^{(n)}, g_{2}^{(n)}} \mathrm{E}\left[g_{1}^{(n)}\left(\mathbf{S}_{1}\right) g_{2}^{(n)}\left(\mathbf{S}_{2}\right)\right] \\
& \stackrel{a)}{\leq} \sup _{\substack{1 \leq k \leq n \\
g_{1, k}, g_{2, k}}} \mathrm{E}\left[g_{1, k}\left(S_{1, k}\right) g_{2, k}\left(S_{2, k}\right)\right] \\
& \leq \rho) \\
& \leq \rho,
\end{aligned}
$$

where $a$ ) follows from Theorem C.1 and $b$ ) follows from Lemma C.3 and from our assumption that $\rho \geq 0$ (Reduction 2.1). Thus, for every time $k$,

$$
\mathrm{E}\left[X_{1, k} X_{2, k}\right] \leq \rho \sqrt{\mathrm{E}\left[X_{1, k}^{2}\right]} \sqrt{\mathrm{E}\left[X_{2, k}^{2}\right]},
$$

which is the bound of Lemma C.2.

Using Lemma C.2 we can now prove the bound of Lemma C.1 as follows:

$$
\begin{aligned}
\frac{1}{n} \sum_{k=1}^{n} \mathrm{E}\left[\left(X_{1, k}+X_{2, k}\right)^{2}\right] & =\frac{1}{n} \sum_{k=1}^{n} \mathrm{E}\left[X_{1, k}^{2}\right]+\frac{1}{n} \sum_{k=1}^{n} \mathrm{E}\left[X_{1, k}^{2}\right]+2 \frac{1}{n} \sum_{k=1}^{n} \mathrm{E}\left[X_{1, k} X_{2, k}\right] \\
& \leq P_{1}+P_{2}+2 \frac{1}{n} \sum_{k=1}^{n} \mathrm{E}\left[X_{1, k} X_{2, k}\right] \\
& \leq P_{1}+P_{2}+2 \rho \frac{1}{n} \sum_{k=1}^{n} \sqrt{\mathrm{E}\left[X_{1, k}^{2}\right]} \sqrt{\mathrm{E}\left[X_{2, k}^{2}\right]} \\
& \leq P_{1}+P_{2}+2 \rho \frac{1}{n} \sqrt{\sum_{k=1}^{n} \mathrm{E}\left[X_{1, k}^{2}\right]} \sqrt{\sum_{k=1}^{n} \mathrm{E}\left[X_{2, k}^{2}\right]} \\
& \leq P_{1}+P_{2}+2 \rho \frac{1}{n} \sqrt{n P_{1}} \sqrt{n P_{2}} \\
& =P_{1}+P_{2}+2 \rho \sqrt{P_{1} P_{2}},
\end{aligned}
$$

where Inequality $a$ ) follows by Lemma C.2 and from our assumption that $\rho \geq 0$, and where Inequality $b$ ) follows by Cauchy-Schwarz. This concludes the proof of Lemma C.1.

\section{Distortions $\left(D_{1}^{\mathrm{u}}, D_{2}^{\mathrm{u}}\right)$ of the Uncoded Scheme}

The expression for $D_{i}^{\mathrm{u}}, i \in\{1,2\}$, is obtained as follows

$$
D_{i}^{\mathrm{u}}=\frac{1}{n} \sum_{k=1}^{n} \mathrm{E}\left[\left(S_{i, k}-\hat{S}_{i, k}^{\mathrm{u}}\right)^{2}\right]
$$




$$
\begin{aligned}
& =\frac{1}{n}\left(\mathrm{E}\left[S_{i, k}^{2}\right]-2 \mathrm{E}\left[S_{i, k} \hat{S}_{i, k}^{\mathrm{u}}\right]+\mathrm{E}\left[\left(\hat{S}_{i, k}^{\mathrm{u}}\right)^{2}\right]\right) \\
& \stackrel{\text { a) }}{=} \frac{1}{n}\left(\mathrm{E}\left[S_{i, k}^{2}\right]-\mathrm{E}\left[\left(\hat{S}_{i, k}^{\mathrm{u}}\right)^{2}\right]\right) \\
& \stackrel{b)}{=} \frac{1}{n}\left(\mathrm{E}\left[S_{i, k}^{2}\right]-\frac{\left(\mathrm{E}\left[S_{i, k} Y_{k}\right]\right)^{2}}{\mathrm{E}\left[Y_{k}^{2}\right]}\right) \\
& \stackrel{c)}{=} \sigma^{2}-\sigma^{2} \frac{P_{1}+2 \rho \sqrt{P_{1} P_{2}}+\rho^{2} P_{2}}{P_{1}+2 \rho \sqrt{P_{1} P_{2}}+P_{2}+N} \\
& =\sigma^{2} \frac{P_{1}\left(1-\rho^{2}\right)+N}{P_{1}+P_{2}+2 \rho \sqrt{P_{1} P_{2}}+N},
\end{aligned}
$$

where in $a$ ) we have used that $\hat{S}_{i, k}^{\mathrm{u}}=\mathrm{E}\left[S_{i, k} \mid Y_{k}\right]$ satisfies the Orthogonality Principle; in $b$ ) we have used the explicit form of the conditional mean for jointly Gaussians

$$
\hat{S}_{i, k}^{\mathrm{u}}=\mathrm{E}\left[S_{i, k} \mid Y_{k}\right]=\frac{\mathrm{E}\left[S_{1, k} Y_{k}\right]}{\mathrm{E}\left[Y_{k}^{2}\right]} \cdot Y_{k}
$$

and in $c$ ) we have used the calculation

$$
\frac{\left(\mathrm{E}\left[S_{1, k} Y_{k}\right]\right)^{2}}{\mathrm{E}\left[Y_{k}^{2}\right]}=\frac{\sigma^{2}\left(\sqrt{P_{1}}+\rho \sqrt{P_{2}}\right)^{2}}{P_{1}+P_{2}+2 \rho \sqrt{P_{1} P_{2}}+N} .
$$

\section{E Proof of Theorem 4.4}

In this appendix we analyze the distortions achievable by the vector-quantizer scheme that was presented in Section 4.4. To start, we give a thorough description of the corresponding coding scheme.

\section{E.1 Coding Scheme}

Fix some $\epsilon>0$ and rates $R_{1}$ and $R_{2}$.

Code Construction: Two codebooks $\mathcal{C}_{1}$ and $\mathcal{C}_{2}$ are generated independently. Codebook $\mathcal{C}_{i}, i \in\{1,2\}$, consists of $2^{n R_{i}}$ codewords $\left\{\mathbf{U}_{i}(1), \mathbf{U}_{i}(2), \ldots, \mathbf{U}_{i}\left(2^{n R_{i}}\right)\right\}$. The codewords are drawn independently uniformly over the surface of the centered $\mathbb{R}^{n}$-sphere $\mathcal{S}_{i}$ of radius $r_{i}=\sqrt{n \sigma^{2}\left(1-2^{-2 R_{i}}\right)}$.

Encoding: Based on the observed source sequence $\mathbf{S}_{i}$ each encoder produces its channel input $\mathbf{X}_{i}$ by first vector-quantizing the source sequence $\mathbf{S}_{i}$ to a codeword $\mathbf{U}_{i}^{*} \in \mathcal{C}_{i}$ and then scaling $\mathbf{U}_{i}^{*}$ to satisfy the average power constraint. To describe the vectorquantizer precisely, denote for every $\mathbf{w}, \mathbf{v} \in \mathbb{R}^{n}$ where neither $\mathbf{w}$ nor $\mathbf{v}$ are the zerosequence, the angle between $\mathbf{w}$ and $\mathbf{v}$ by $\varangle(\mathbf{w}, \mathbf{v})$, i.e.

$$
\cos \varangle(\mathbf{w}, \mathbf{v}) \triangleq \frac{\langle\mathbf{w}, \mathbf{v}\rangle}{\|\mathbf{w}\|\|\mathbf{v}\|} .
$$


Let

$$
\mathcal{F}\left(\mathbf{s}_{i}, \mathcal{C}_{i}\right)=\left\{\mathbf{u}_{i} \in \mathcal{C}_{i}: \sqrt{1-2^{-2 R_{i}}}(1-\epsilon) \leq \cos \varangle\left(\mathbf{s}_{i}, \mathbf{U}_{i}\right) \leq \sqrt{1-2^{-2 R_{i}}}(1+\epsilon)\right\} .
$$

If $\mathcal{F}\left(\mathbf{s}_{i}, \mathcal{C}_{i}\right) \neq \emptyset$, the vector-quantizer output $\mathbf{U}_{i}^{*}$ is the codeword $\mathbf{U}_{i}(j) \in \mathcal{F}\left(\mathbf{s}_{i}, \mathcal{C}_{i}\right)$ that minimizes $\left|\cos \varangle\left(\mathbf{u}_{i}(j), \mathbf{s}_{i}\right)-\sqrt{1-2^{-2 R_{i}}}\right|$, and if $\mathcal{F}\left(\mathbf{s}_{i}, \mathcal{C}_{i}\right)=\emptyset$ the vector-quantizer output $\mathbf{U}_{i}^{*}$ is the all-zero sequence. Thus,

$$
\mathbf{U}_{i}^{*}= \begin{cases}\underset{\mathbf{0}}{\operatorname{argmin}} \underset{\mathbf{U}_{i} \in \mathcal{F}\left(\mathbf{s}_{i}, \mathcal{C}_{i}\right)}{ }\left|\cos \varangle\left(\mathbf{u}_{i}(j), \mathbf{S}_{i}\right)-\sqrt{1-2^{-2 R_{i}}}\right| & \text { if } \mathcal{F}\left(\mathbf{s}_{i}, \mathcal{C}_{i}\right) \neq \emptyset, \\ \mathbf{0} & \text { otherwise. }\end{cases}
$$

More formally, $\mathbf{U}_{i}^{*}$ should be written as $\mathbf{U}_{i}^{*}\left(\mathbf{S}_{i}, \mathcal{C}_{i}\right)$, but we shall usually make these dependencies implicit. The channel input is now given by

$$
\mathbf{X}_{i}=\alpha_{i} \mathbf{U}_{i}^{*} \quad \text { where } \alpha_{i}=\sqrt{\frac{P_{i}}{\sigma^{2}\left(1-2^{-2 R_{i}}\right)}} \quad i \in\{1,2\} .
$$

Since the codebook $\mathcal{C}_{i}$ is drawn over the centered $\mathbb{R}^{n}$-sphere of radius $r_{i}=\sqrt{\sigma^{2}\left(1-2^{-2 R_{i}}\right)}$, each channel input $\mathbf{X}_{i}$ satisfies the average power constraint individually.

Reconstruction: The receiver's estimate $\left(\hat{\mathbf{S}}_{1}, \hat{\mathbf{S}}_{2}\right)$ of the source pair $\left(\mathbf{S}_{1}, \mathbf{S}_{2}\right)$ is derived from the channel output $\mathbf{Y}$ in two steps. First, the receiver makes a guess $\left(\hat{\mathbf{U}}_{1}, \hat{\mathbf{U}}_{2}\right)$ of the pair $\left(\mathbf{U}_{1}^{*}, \mathbf{U}_{2}^{*}\right)$ by choosing among all "jointly typical pairs" $\left(\mathbf{U}_{1}, \mathbf{U}_{2}\right) \in \mathcal{C}_{1} \times \mathcal{C}_{2}$ the pair whose linear combination $\alpha_{1} \mathbf{U}_{1}+\alpha_{2} \mathbf{U}_{2}$ has the smallest distance to the received sequence $\mathbf{Y}$. More precisely,

$$
\left(\hat{\mathbf{U}}_{1}, \hat{\mathbf{U}}_{2}\right)=\underset{\substack{\left(\mathbf{U}_{1}, \mathbf{U}_{2}\right) \in \mathcal{C}_{1} \times \mathcal{C}_{2}: \\\left|\tilde{\rho}-\cos \varangle\left(\mathbf{u}_{1}, \mathbf{u}_{2}\right)\right| \leq 7 \epsilon}}{\operatorname{argmin}}\left\|\mathbf{Y}-\left(\alpha_{1} \mathbf{U}_{1}+\alpha_{2} \mathbf{U}_{2}\right)\right\|^{2},
$$

where

$$
\tilde{\rho}=\rho \sqrt{\left(1-2^{-2 R_{1}}\right)\left(1-2^{-2 R_{2}}\right)} .
$$

If the channel output $\mathbf{Y}$ and the codebooks $\mathcal{C}_{1}$ and $\mathcal{C}_{2}$ are such that there does not exist a pair $\left(\mathbf{U}_{1}, \mathbf{U}_{2}\right) \in \mathcal{C}_{1} \times \mathcal{C}_{2}$ that satisfies

$$
\left|\tilde{\rho}-\cos \varangle\left(\mathbf{u}_{1}, \mathbf{u}_{2}\right)\right| \leq 7 \epsilon,
$$

then $\hat{\mathbf{U}}_{1}$ and $\hat{\mathbf{U}}_{2}$ are chosen to be all-zero.

In the second step, the receiver computes the estimates $\left(\hat{\mathbf{S}}_{1}, \hat{\mathbf{S}}_{2}\right)$ from the guess $\left(\hat{\mathbf{U}}_{1}, \hat{\mathbf{U}}_{2}\right)$ by setting

$$
\begin{aligned}
& \hat{\mathbf{S}}_{1}=\gamma_{11} \hat{\mathbf{U}}_{1}+\gamma_{12} \hat{\mathbf{U}}_{2} \\
& \hat{\mathbf{S}}_{2}=\gamma_{21} \hat{\mathbf{U}}_{2}+\gamma_{22} \hat{\mathbf{U}}_{1},
\end{aligned}
$$

where

$$
\begin{aligned}
\gamma_{11} & =\frac{1-\rho^{2}\left(1-2^{-2 R_{2}}\right)}{1-\tilde{\rho}^{2}} & \gamma_{12} & =\rho 2^{-2 R_{1}} \\
\gamma_{21} & =\frac{1-\rho^{2}\left(1-2^{-2 R_{1}}\right)}{1-\tilde{\rho}^{2}} & \gamma_{22} & =\rho 2^{-2 R_{2}} .
\end{aligned}
$$


Note that

$$
0<\gamma_{i 1} \leq 1 \quad \text { and } \quad 0<\gamma_{i 2} \leq \rho, \quad i \in\{1,2\}
$$

\section{E.2 Expected Distortion}

To analyze the expected distortion we use a genie-aided argument. We first show that, under certain rate constraints, the asymptotic normalized distortion of the proposed scheme remains the same when a certain help from a genie is provided. To derive the achievable distortions it then suffices to analyze the genie-aided version.

\section{E.2.1 Genie-Aided Scheme}

In the genie-aided scheme, the genie's help is provided to the decoder. An illustration of this genie-aided decoder is given in Figure 9. The genie provides the decoder with

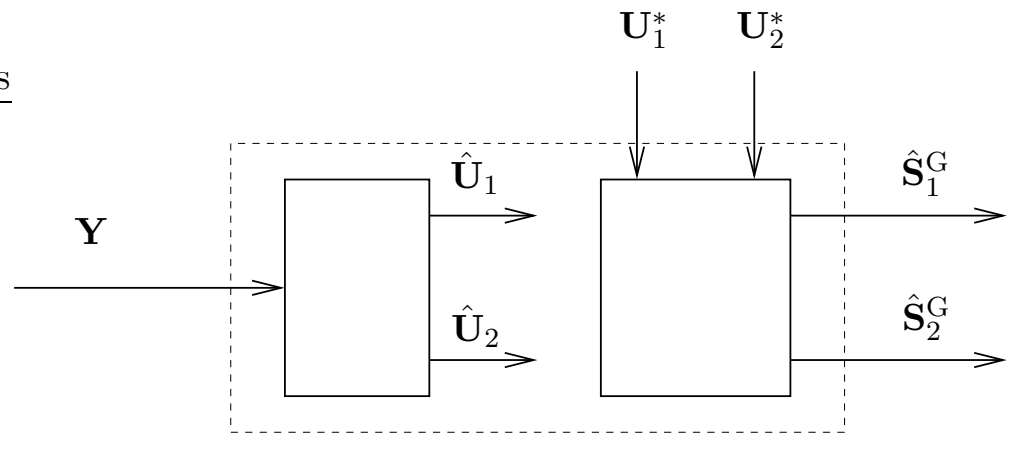

Figure 9: Genie-aided decoder.

the codeword pair $\left(\mathbf{U}_{1}^{*}, \mathbf{U}_{2}^{*}\right)$. The decoder then estimates the source pair $\left(\mathbf{S}_{1}, \mathbf{S}_{2}\right)$ based on $\left(\mathbf{U}_{1}^{*}, \mathbf{U}_{2}^{*}\right)$ and ignores the guess $\left(\hat{\mathbf{U}}_{1}, \hat{\mathbf{U}}_{2}\right)$ produced in the first decoding step. The estimate of this genie-aided decoder is denoted by $\left(\hat{\mathbf{S}}_{1}^{\mathrm{G}}, \hat{\mathbf{S}}_{2}^{\mathrm{G}}\right)$, where

$$
\begin{aligned}
& \hat{\mathbf{S}}_{1}^{\mathrm{G}}=\gamma_{11} \mathbf{U}_{1}^{*}+\gamma_{12} \mathbf{U}_{2}^{*} \\
& \hat{\mathbf{S}}_{2}^{\mathrm{G}}=\gamma_{21} \mathbf{U}_{2}^{*}+\gamma_{22} \mathbf{U}_{1}^{*},
\end{aligned}
$$

with $\gamma_{11}, \gamma_{12}, \gamma_{21}, \gamma_{22}$ as in (84) and (85). Under certain rate constraints, the normalized asymptotic distortion of this genie-aided scheme is the same as for the originally proposed scheme. This is stated more precisely in the following proposition.

Proposition E.1. For every $\delta>0$ and $0<\epsilon<0.3$ there exists an $n^{\prime}(\delta, \epsilon)>0$ such that for all $n>n^{\prime}(\delta, \epsilon)$,

$$
\frac{1}{n} \mathrm{E}\left[\left\|\mathbf{S}_{1}-\hat{\mathbf{S}}_{1}\right\|^{2}\right] \leq \frac{1}{n} \mathrm{E}\left[\left\|\mathbf{S}_{1}-\hat{\mathbf{S}}_{1}^{\mathrm{G}}\right\|^{2}\right]+2 \sigma^{2}(\epsilon+(44 \sqrt{1+\epsilon}+61) \delta) .
$$


whenever $\left(R_{1}, R_{2}\right)$ is in the rate region $\mathcal{R}(\epsilon)$ given by

$$
\begin{aligned}
& \mathcal{R}(\epsilon)=\left\{\quad R_{1} \leq \frac{1}{2} \log _{2}\left(\frac{P_{1}\left(1-\tilde{\rho}^{2}\right)+N}{N\left(1-\tilde{\rho}^{2}\right)}-\kappa_{1} \epsilon\right),\right. \\
& R_{2} \leq \frac{1}{2} \log _{2}\left(\frac{P_{2}\left(1-\tilde{\rho}^{2}\right)+N}{N\left(1-\tilde{\rho}^{2}\right)}-\kappa_{2} \epsilon\right), \\
& \left.R_{1}+R_{2} \leq \frac{1}{2} \log _{2}\left(\frac{P_{1}+P_{2}+2 \tilde{\rho} \sqrt{P_{1} P_{2}}+N}{N\left(1-\tilde{\rho}^{2}\right)}-\kappa_{3} \epsilon\right)\right\},
\end{aligned}
$$

where $\kappa_{1}, \kappa_{2}$ and $\kappa_{3}$ depend only on $P_{1}, P_{2}, N, \zeta_{1}$ and $\zeta_{2}$, where

$$
\zeta_{1}=\frac{N \tilde{\rho}}{P_{1}\left(1-\tilde{\rho}^{2}\right)+N} \sqrt{\frac{P_{1}}{P_{2}}} \quad \text { and } \quad \zeta_{2}=\frac{P_{1}\left(1-\tilde{\rho}^{2}\right)}{P_{1}\left(1-\tilde{\rho}^{2}\right)+N} .
$$

Proof. See Section E.3.

Corollary E.1. If $\left(R_{1}, R_{2}\right)$ satisfy

$$
\begin{aligned}
R_{1} & <\frac{1}{2} \log _{2}\left(\frac{P_{1}\left(1-\tilde{\rho}^{2}\right)+N}{N\left(1-\tilde{\rho}^{2}\right)}\right) \\
R_{2} & <\frac{1}{2} \log _{2}\left(\frac{P_{2}\left(1-\tilde{\rho}^{2}\right)+N}{N\left(1-\tilde{\rho}^{2}\right)}\right) \\
R_{1}+R_{2} & <\frac{1}{2} \log _{2}\left(\frac{P_{1}+P_{2}+2 \tilde{\rho} \sqrt{P_{1} P_{2}}+N}{N\left(1-\tilde{\rho}^{2}\right)}\right),
\end{aligned}
$$

then

$$
\varlimsup_{n \rightarrow \infty} \frac{1}{n} \mathrm{E}\left[\left\|\mathbf{S}_{1}-\hat{\mathbf{S}}_{1}\right\|^{2}\right] \leq \varlimsup_{n \rightarrow \infty} \frac{1}{n} \mathrm{E}\left[\left\|\mathbf{S}_{1}-\hat{\mathbf{S}}_{1}^{\mathrm{G}}\right\|^{2}\right] .
$$

Proof. Follows from Proposition E.1 by first letting $n \rightarrow \infty$ and then $\epsilon \rightarrow 0$ and $\delta \rightarrow 0$.

By Corollary E.1, to analyze the distortion achievable by our scheme it suffices to analyze the genie-aided scheme. This is done in Section E.4.

\section{E.3 Proof of Proposition E.1}

The main step in the proof of Proposition E.1 is to show that for every $\left(R_{1}, R_{2}\right) \in \mathcal{R}(\epsilon)$ and sufficiently large $n$, the probability of a decoding error, and thus the probability of $\hat{\mathbf{S}}_{1} \neq \hat{\mathbf{S}}_{1}^{\mathrm{G}}$, can be made very small. This step is done in the following section. The proof of Proposition E.1 is then completed in Section E.3.2.

\section{E.3.1 Upper Bound on Probability of a Decoding Error}

In this section we show that for every $\left(R_{1}, R_{2}\right) \in \mathcal{R}(\epsilon)$ and sufficiently large $n$, the probability of a decoding error, and thus the probability of $\hat{\mathbf{S}}_{1} \neq \hat{\mathbf{S}}_{1}^{\mathrm{G}}$, can be made very small. The hitch is that to upper bound the probability of a decoding error 
for the proposed scheme, we cannot proceed by the method conventionally used for the multiple-access channel. The reason is that in the conventional analysis of the multiple-access channel it is assumed that the probability of the codewords $\mathbf{u}_{i}(j)$ does not depend on the realization of the codebook $\mathcal{C}_{i}$. However, in our combined sourcechannel coding scheme, the probability of encoder $i \in\{1,2\}$ producing the channel input of index $j \in\left\{1,2, \ldots, 2^{n R_{i}}\right\}$ depends not only on the source sequence $\mathbf{s}_{i}$, but also on the realization of $\mathcal{C}_{i}$. Another reason the conventional analysis fails is that, conditional on the codebooks $\mathcal{C}_{1}$ and $\mathcal{C}_{2}$, the indices produces by the vector-quantizers are dependent.

To address these difficulties, we proceed by a geometric approach. To this end, we introduce an error event related to a decoding error at the receiver. This event is denoted by $\mathcal{E}_{\hat{\mathbf{U}}}$ and consists of all tuples $\left(\mathbf{s}_{1}, \mathbf{s}_{2}, \mathcal{C}_{1}, \mathcal{C}_{2}, \mathbf{z}\right)$ for which there exists a pair $\left(\tilde{\mathbf{u}}_{1}, \tilde{\mathbf{u}}_{2}\right) \neq\left(\mathbf{u}_{1}^{*}, \mathbf{u}_{2}^{*}\right)$ in $\mathcal{C}_{1} \times \mathcal{C}_{2}$ that satisfies Condition (81) of the reconstructor, and for which the Euclidean distance between $\alpha_{1} \tilde{\mathbf{u}}_{1}+\alpha_{2} \tilde{\mathbf{u}}_{2}$ and $\mathbf{y}$ is smaller or equal to the Euclidean distance between $\alpha_{1} \mathbf{u}_{1}^{*}+\alpha_{2} \mathbf{u}_{2}^{*}$ and $\mathbf{y}$. More formally, $\mathcal{E}_{\hat{\mathbf{U}}}=\mathcal{E}_{\hat{\mathbf{U}}_{1}} \cup \mathcal{E}_{\hat{\mathbf{U}}_{2}} \cup$ $\mathcal{E}_{\left(\hat{\mathbf{U}}_{1}, \hat{\mathbf{U}}_{2)}\right)}$ where

$$
\begin{gathered}
\mathcal{E}_{\hat{\mathbf{U}}_{1}}=\left\{\left(\mathbf{s}_{1}, \mathbf{s}_{2}, \mathcal{C}_{1}, \mathcal{C}_{2}, \mathbf{z}\right): \exists \tilde{\mathbf{u}}_{1} \in \mathcal{C}_{1} \backslash\left\{\mathbf{u}_{1}^{*}\right\} \text { s.t. }\left|\tilde{\rho}-\cos \varangle\left(\tilde{\mathbf{u}}_{1}, \mathbf{u}_{2}^{*}\right)\right| \leq 7 \epsilon,\right. \\
\text { and } \left.\left\|\mathbf{y}-\left(\alpha_{1} \tilde{\mathbf{u}}_{1}+\alpha_{2} \mathbf{u}_{2}^{*}\right)\right\|^{2} \leq\left\|\mathbf{y}-\left(\alpha_{1} \mathbf{u}_{1}^{*}+\alpha_{2} \mathbf{u}_{2}^{*}\right)\right\|^{2}\right\} \\
\mathcal{E}_{\hat{\mathbf{U}}_{2}}=\left\{\left(\mathbf{s}_{1}, \mathbf{s}_{2}, \mathcal{C}_{1}, \mathcal{C}_{2}, \mathbf{z}\right): \exists \tilde{\mathbf{u}}_{2} \in \mathcal{C}_{2} \backslash\left\{\mathbf{u}_{2}^{*}\right\} \text { s.t. }\left|\tilde{\rho}-\cos \varangle\left(\mathbf{u}_{1}^{*}, \tilde{\mathbf{u}}_{2}\right)\right| \leq 7 \epsilon,\right. \\
\text { and } \left.\left\|\mathbf{y}-\left(\alpha_{1} \mathbf{u}_{1}^{*}+\alpha_{2} \tilde{\mathbf{u}}_{2}\right)\right\|^{2} \leq\left\|\mathbf{y}-\left(\alpha_{1} \mathbf{u}_{1}^{*}+\alpha_{2} \mathbf{u}_{2}^{*}\right)\right\|^{2}\right\} \\
\mathcal{E}_{\left(\hat{\mathbf{U}}_{1}, \hat{\mathbf{U}}_{2}\right)}=\left\{\left(\mathbf{s}_{1}, \mathbf{s}_{2}, \mathcal{C}_{1}, \mathcal{C}_{2}, \mathbf{z}\right): \exists \tilde{\mathbf{u}}_{1} \in \mathcal{C}_{1} \backslash\left\{\mathbf{u}_{1}^{*}\right\} \text { and } \exists \tilde{\mathbf{u}}_{2} \in \mathcal{C}_{2} \backslash\left\{\mathbf{u}_{2}^{*}\right\}\right. \text { s.t. } \\
\left|\tilde{\rho}-\cos \varangle\left(\tilde{\mathbf{u}}_{1}, \tilde{\mathbf{u}}_{2}\right)\right| \leq 7 \epsilon, \\
\text { and } \left.\left\|\mathbf{y}-\left(\alpha_{1} \tilde{\mathbf{u}}_{1}+\alpha_{2} \tilde{\mathbf{u}}_{2}\right)\right\|^{2} \leq\left\|\mathbf{y}-\left(\alpha_{1} \mathbf{u}_{1}^{*}+\alpha_{2} \mathbf{u}_{2}^{*}\right)\right\|^{2}\right\},
\end{gathered}
$$

and where $\mathbf{y} \triangleq \alpha_{1} \mathbf{u}_{1}^{*}+\alpha_{1} \mathbf{u}_{1}^{*}+\mathbf{z}$. Note that a decoding error occurs only if $\left(\mathbf{s}_{1}, \mathbf{s}_{2}, \mathcal{C}_{1}, \mathcal{C}_{2}, \mathbf{z}\right) \in$ $\mathcal{E}_{\hat{\mathbf{U}}}$. The main result of this section can now be stated as follows.

Lemma E.1. For every $\delta>0$ and $0.3>\epsilon>0$, there exists an $n_{4}^{\prime}(\delta, \epsilon) \in \mathbb{N}$ such that for all $n>n_{4}^{\prime}(\delta, \epsilon)$

$$
\operatorname{Pr}\left[\mathcal{E}_{\hat{\mathbf{U}}}\right]<11 \delta, \quad \text { whenever }\left(R_{1}, R_{2}\right) \in \mathcal{R}(\epsilon) .
$$

To prove Lemma E.1, we introduce three auxiliary error events. The first auxiliary event $\mathcal{E}_{\mathbf{S}}$ corresponds to an atypical source output. More precisely,

$$
\begin{aligned}
& \mathcal{E}_{\mathbf{S}}=\left\{\left(\mathbf{s}_{1}, \mathbf{s}_{2}\right) \in \mathbb{R}^{n} \times \mathbb{R}^{n}:\left|\frac{1}{n}\left\|\mathbf{s}_{1}\right\|^{2}-\sigma^{2}\right|>\epsilon \sigma^{2} \quad \text { or } \quad\left|\frac{1}{n}\left\|\mathbf{s}_{2}\right\|^{2}-\sigma^{2}\right|>\epsilon \sigma^{2}\right. \\
& \text { or } \left.\left|\cos \varangle\left(\mathbf{s}_{1}, \mathbf{s}_{2}\right)-\rho\right|>\epsilon \rho\right\} \text {. }
\end{aligned}
$$


The second auxiliary event is denoted by $\mathcal{E}_{\mathbf{Z}}$ and corresponds to an atypical behavior of the additive noise:

$$
\begin{aligned}
\mathcal{E}_{\mathbf{Z}}=\left\{\left(\mathbf{s}_{1}, \mathbf{s}_{2}, \mathcal{C}_{1}, \mathcal{C}_{2}, \mathbf{z}\right):\left|\frac{1}{n}\|\mathbf{z}\|^{2}-N\right|>\epsilon N\right. & \text { or } \frac{1}{n}\left|\left\langle\alpha_{1} \mathbf{u}_{1}^{*}\left(\mathbf{s}_{1}, \mathcal{C}_{1}\right), \mathbf{z}\right\rangle\right|>\sqrt{P_{1} N} \epsilon \\
& \text { or } \left.\frac{1}{n}\left|\left\langle\alpha_{2} \mathbf{u}_{2}^{*}\left(\mathbf{s}_{2}, \mathcal{C}_{2}\right), \mathbf{z}\right\rangle\right|>\sqrt{P_{2} N} \epsilon\right\} .
\end{aligned}
$$

Finally, the third auxiliary event is denoted by $\mathcal{E}_{\mathbf{X}}$ and corresponds to irregularities at the encoders. That is, the event that one of the codebooks contains no codeword satisfying Condition (78) of the vector-quantizer, or that the two quantized sequences $\mathbf{u}_{1}^{*}$ and $\mathbf{u}_{2}^{*}$ have an "atypical" angle to each other. More formally, $\mathcal{E}_{\mathbf{X}}=\mathcal{E}_{\mathbf{X}_{1}} \cup \mathcal{E}_{\mathbf{X}_{2}} \cup \mathcal{E}_{\left(\mathbf{X}_{1}, \mathbf{X}_{2}\right)}$ where

$$
\begin{aligned}
& \mathcal{E}_{\mathbf{X}_{1}}=\left\{\left(\mathbf{s}_{1}, \mathbf{s}_{2}, \mathcal{C}_{1}, \mathcal{C}_{2}\right): \nexists \mathbf{u}_{1} \in \mathcal{C}_{1} \text { s.t. }\left|\sqrt{1-2^{-2 R_{1}}}-\cos \varangle\left(\mathbf{s}_{1}, \mathbf{u}_{1}\right)\right| \leq \epsilon \sqrt{1-2^{-2 R_{1}}}\right\} \\
& \mathcal{E}_{\mathbf{X}_{2}}=\left\{\left(\mathbf{s}_{1}, \mathbf{s}_{2}, \mathcal{C}_{1}, \mathcal{C}_{2}\right): \nexists \mathbf{u}_{2} \in \mathcal{C}_{2} \text { s.t. }\left|\sqrt{1-2^{-2 R_{2}}}-\cos \varangle\left(\mathbf{s}_{2}, \mathbf{u}_{2}\right)\right| \leq \epsilon \sqrt{1-2^{-2 R_{2}}}\right\} \\
& \mathcal{E}_{\left(\mathbf{X}_{1}, \mathbf{X}_{2}\right)}=\left\{\left(\mathbf{s}_{1}, \mathbf{s}_{2}, \mathcal{C}_{1}, \mathcal{C}_{2}\right):\left|\tilde{\rho}-\cos \varangle\left(\mathbf{u}_{1}^{*}\left(\mathbf{s}_{1}, \mathcal{C}_{1}\right), \mathbf{u}_{2}^{*}\left(\mathbf{s}_{2}, \mathcal{C}_{2}\right)\right)\right|>7 \epsilon\right\} .
\end{aligned}
$$

To prove Lemma E.1 we now start with the decomposition

$$
\begin{aligned}
\operatorname{Pr}\left[\mathcal{E}_{\hat{\mathbf{U}}}\right]= & \operatorname{Pr}\left[\mathcal{E}_{\hat{\mathbf{U}}} \cap \mathcal{E}_{\mathbf{S}}^{c} \cap \mathcal{E}_{\mathbf{X}}^{c} \cap \mathcal{E}_{\mathbf{Z}}^{c}\right]+\operatorname{Pr}\left[\mathcal{E}_{\hat{\mathbf{U}}} \mid \mathcal{E}_{\mathbf{S}} \cup \mathcal{E}_{\mathbf{X}} \cup \mathcal{E}_{\mathbf{Z}}\right] \operatorname{Pr}\left[\mathcal{E}_{\mathbf{S}} \cup \mathcal{E}_{\mathbf{X}} \cup \mathcal{E}_{\mathbf{Z}}\right] \\
\leq & \operatorname{Pr}\left[\mathcal{E}_{\hat{\mathbf{U}}} \cap \mathcal{E}_{\mathbf{S}}^{c} \cap \mathcal{E}_{\mathbf{X}}^{c} \cap \mathcal{E}_{\mathbf{Z}}^{c}\right]+\operatorname{Pr}\left[\mathcal{E}_{\mathbf{S}}\right]+\operatorname{Pr}\left[\mathcal{E}_{\mathbf{X}}\right]+\operatorname{Pr}\left[\mathcal{E}_{\mathbf{Z}}\right] \\
\leq & \operatorname{Pr}\left[\mathcal{E}_{\hat{\mathbf{U}}_{1}} \cap \mathcal{E}_{\mathbf{S}}^{c} \cap \mathcal{E}_{\mathbf{X}}^{c} \cap \mathcal{E}_{\mathbf{Z}}^{c}\right]+\operatorname{Pr}\left[\mathcal{E}_{\hat{\mathbf{U}}_{2}} \cap \mathcal{E}_{\mathbf{S}}^{c} \cap \mathcal{E}_{\mathbf{X}}^{c} \cap \mathcal{E}_{\mathbf{Z}}^{c}\right] \\
& +\operatorname{Pr}\left[\mathcal{E}_{\left(\hat{\mathbf{U}}_{1}, \hat{\mathbf{U}}_{2}\right)} \cap \mathcal{E}_{\mathbf{S}}^{c} \cap \mathcal{E}_{\mathbf{X}}^{c} \cap \mathcal{E}_{\mathbf{Z}}^{c}\right]+\operatorname{Pr}\left[\mathcal{E}_{\mathbf{S}}\right]+\operatorname{Pr}\left[\mathcal{E}_{\mathbf{X}}\right]+\operatorname{Pr}\left[\mathcal{E}_{\mathbf{Z}}\right]
\end{aligned}
$$

where we have used the shorthand notation $\operatorname{Pr}\left[\mathcal{E}_{\nu}\right]$ for $\operatorname{Pr}\left[\left(\mathbf{S}_{1}, \mathbf{S}_{2}, \mathcal{C}_{1}, \mathcal{C}_{2}, \mathbf{Z}\right) \in \mathcal{E}_{\nu}\right]$, and where $\mathcal{E}_{\nu}^{c}$ denotes the complement of $\mathcal{E}_{\nu}$. Lemma E.1 now follows from upper bounding the probability terms on the RHS of (94).

Lemma E.2. For every $\delta>0$ and $\epsilon>0$ there exists an $n_{1}^{\prime}(\delta, \epsilon) \in \mathbb{N}$ such that for all $n>n_{1}^{\prime}(\delta, \epsilon)$

$$
\operatorname{Pr}\left[\mathcal{E}_{\mathbf{S}}\right]<\delta .
$$

Proof. The proof follows by the weak law of large numbers.

Lemma E.3. For every $\epsilon>0$ and $\delta>0$ there exists an $n_{3}^{\prime}(\delta, \epsilon) \in \mathbb{N}$ such that for all $n>n_{3}^{\prime}(\delta, \epsilon)$

$$
\operatorname{Pr}\left[\mathcal{E}_{\mathbf{Z}}\right]<\delta
$$

Proof. The proof follows by the weak law of large numbers and since for every $\epsilon>0$

$$
\sup _{\|\mathbf{u}\|=\sqrt{n \mathbb{R}^{n}:}} \operatorname{Pr}\left[\frac{1}{n}\left|\left\langle\alpha_{i} \mathbf{u}, \mathbf{z}\right\rangle\right|>\sqrt{P_{i} N} \epsilon\right] \rightarrow 0 \quad \text { as } n \rightarrow \infty,
$$

where $i \in\{1,2\}$. 
Lemma E.4. For every $\delta>0$ and $0.3>\epsilon>0$ there exists an $n_{2}^{\prime}(\delta, \epsilon) \in \mathbb{N}$ such that for all $n>n_{2}^{\prime}(\delta, \epsilon)$

$$
\operatorname{Pr}\left[\mathcal{E}_{\mathbf{X}}\right]<6 \delta
$$

Proof. This result has nothing to do with the channel; it is a result from rate-distortion theory. A proof for our setting is given in Section E.5.1.

Lemma E.5. For every $\delta>0$ and every $\epsilon>0$ there exists some $n_{4}^{\prime \prime}(\delta, \epsilon) \in \mathbb{N}$ such that for all $n>n_{4}^{\prime \prime}(\delta, \epsilon)$

$$
\begin{aligned}
& \operatorname{Pr}\left[\mathcal{E}_{\hat{\mathbf{U}}_{1}} \cap \mathcal{E}_{\mathbf{S}}^{c} \cap \mathcal{E}_{\mathbf{X}}^{c} \cap \mathcal{E}_{\mathbf{Z}}^{c}\right] \leq \delta, \quad \text { if } R_{1}<\frac{1}{2} \log _{2}\left(\frac{P_{1}\left(1-\tilde{\rho}^{2}\right)+N}{N\left(1-\tilde{\rho}^{2}\right)}-\kappa_{1} \epsilon\right) \\
& \operatorname{Pr}\left[\mathcal{E}_{\hat{\mathbf{U}}_{2}} \cap \mathcal{E}_{\mathbf{S}}^{c} \cap \mathcal{E}_{\mathbf{X}}^{c} \cap \mathcal{E}_{\mathbf{Z}}^{c}\right] \leq \delta, \quad \text { if } R_{2}<\frac{1}{2} \log _{2}\left(\frac{P_{2}\left(1-\tilde{\rho}^{2}\right)+N}{N\left(1-\tilde{\rho}^{2}\right)}-\kappa_{2} \epsilon\right) \\
& \operatorname{Pr}\left[\mathcal{E}_{\left(\hat{\mathbf{U}}_{1}, \hat{\mathbf{U}}_{2}\right)} \cap \mathcal{E}_{\mathbf{S}}^{c} \cap \mathcal{E}_{\mathbf{X}}^{c} \cap \mathcal{E}_{\mathbf{Z}}^{c}\right] \leq \delta, \text { if } R_{1}+R_{2}<\frac{1}{2} \log _{2}\left(\frac{P_{1}+P_{2}+2 \tilde{\rho} \sqrt{P_{1} P_{2}}+N}{N\left(1-\tilde{\rho}^{2}\right)}-\kappa_{3} \epsilon\right),
\end{aligned}
$$

where $\kappa_{1}, \kappa_{2}$, and $\kappa_{3}$ are positive constants determined by $P_{1}, P_{2}$, and $N$.

The proof of Lemma E.5 requires some preliminaries. To this end, define

$$
\mathbf{w}\left(\mathbf{s}_{1}, \mathbf{s}_{2}, \mathcal{C}_{1}, \mathcal{C}_{2}, \mathbf{z}\right)=\zeta_{1}\left(\mathbf{y}-\alpha_{2} \mathbf{u}_{2}^{*}\right)+\zeta_{2} \alpha_{2} \mathbf{u}_{2}^{*}
$$

where

$$
\zeta_{1}=\frac{N \tilde{\rho}}{P_{1}\left(1-\tilde{\rho}^{2}\right)+N} \sqrt{\frac{P_{1}}{P_{2}}} \quad \text { and } \quad \zeta_{2}=\frac{P_{1}\left(1-\tilde{\rho}^{2}\right)}{P_{1}\left(1-\tilde{\rho}^{2}\right)+N}
$$

In the remainder we shall use the shorthand notation $\mathbf{w}$ instead of $\mathbf{w}\left(\mathbf{s}_{1}, \mathbf{s}_{2}, \mathcal{C}_{1}, \mathcal{C}_{2}, \mathbf{z}\right)$. We now start with a lemma that will be used to prove (95).

Lemma E.6. Let $\varphi_{j} \in[0, \pi]$ be the angle between $\mathbf{w}$ and $\mathbf{u}_{1}(j)$, and let the set $\mathcal{E}_{\hat{\mathbf{U}}_{1}}^{\prime}$ be defined as

$$
\begin{aligned}
\mathcal{E}_{\hat{\mathbf{U}}_{1}}^{\prime} \triangleq\left\{\left(\mathbf{s}_{1}, \mathbf{s}_{2}, \mathcal{C}_{1}, \mathcal{C}_{2}, \mathbf{z}\right): \exists \mathbf{u}_{1}(j)\right. & \in \mathcal{C}_{1} \backslash\left\{\mathbf{u}_{1}^{*}\right\} \text { s.t. } \\
\cos \varphi_{j} & \left.\geq \sqrt{\frac{P_{1}\left(1-\tilde{\rho}^{2}\right)+N \tilde{\rho}^{2}}{P_{1}\left(1-\tilde{\rho}^{2}\right)+N}-\kappa^{\prime \prime} \epsilon}\right\},
\end{aligned}
$$

where $\kappa^{\prime \prime}$ is a positive constant determined by $P_{1}, P_{2}, N, \zeta_{1}$ and $\zeta_{2}$. Then,

$$
\mathcal{E}_{\hat{\mathbf{U}}_{1}} \cap \mathcal{E}_{\mathbf{S}}^{c} \cap \mathcal{E}_{\mathbf{X}}^{c} \cap \mathcal{E}_{\mathbf{Z}}^{c} \subseteq \mathcal{E}_{\hat{\mathbf{U}}_{1}}^{\prime} \cap \mathcal{E}_{\mathbf{S}}^{c} \cap \mathcal{E}_{\mathbf{X}}^{c} \cap \mathcal{E}_{\mathbf{Z}}^{c}
$$

and, in particular

$$
\operatorname{Pr}\left[\mathcal{E}_{\hat{\mathbf{U}}_{1}} \cap \mathcal{E}_{\mathbf{S}}^{c} \cap \mathcal{E}_{\mathbf{X}}^{c} \cap \mathcal{E}_{\mathbf{Z}}^{c}\right] \leq \operatorname{Pr}\left[\mathcal{E}_{\hat{\mathbf{U}}_{1}}^{\prime} \cap \mathcal{E}_{\mathbf{S}}^{c} \cap \mathcal{E}_{\mathbf{X}}^{c} \cap \mathcal{E}_{\mathbf{Z}}^{c}\right] .
$$


Proof. We first recall that for the event $\mathcal{E}_{\hat{\mathbf{U}}_{1}}$ to occur, there must exist a codeword $\mathbf{u}_{1}(j) \in \mathcal{C}_{1} \backslash\left\{\mathbf{u}_{1}^{*}\right\}$ that satisfies

$$
\left|\tilde{\rho}-\cos \varangle\left(\mathbf{u}_{1}(j), \mathbf{u}_{2}^{*}\right)\right|<7 \epsilon,
$$

and

$$
\left\|\mathbf{y}-\left(\alpha_{1} \mathbf{u}_{1}(j)+\alpha_{2} \mathbf{u}_{2}^{*}\right)\right\|^{2} \leq\left\|\mathbf{y}-\left(\alpha_{1} \mathbf{u}_{1}^{*}+\alpha_{2} \mathbf{u}_{2}^{*}\right)\right\|^{2} .
$$

The proof is now based on a sequence of statements related to Condition (101) and Condition (102):

A) For every $\left(\mathbf{s}_{1}, \mathbf{s}_{2}, \mathcal{C}_{1}, \mathcal{C}_{2}, \mathbf{z}\right) \in \mathcal{E}_{\mathbf{X}}^{c}$ and every $\mathbf{u} \in \mathcal{S}_{1}$, where $\mathcal{S}_{1}$ is the surface area of the codeword sphere of $\mathcal{C}_{1}$ defined in the code construction,

$$
\left|\tilde{\rho}-\cos \varangle\left(\mathbf{u}, \mathbf{u}_{2}^{*}\right)\right|<7 \epsilon \quad \Rightarrow \quad n \tilde{\rho} \sqrt{P_{1} P_{2}}-\left\langle\alpha_{1} \mathbf{u}, \alpha_{2} \mathbf{u}_{2}^{*}\right\rangle \mid \leq 7 n \sqrt{P_{1} P_{2}} \epsilon .
$$

Statement A) follows by rewriting $\cos \varangle\left(\mathbf{u}, \mathbf{u}_{2}^{*}\right)$ as $\left\langle\mathbf{u}, \mathbf{u}_{2}^{*}\right\rangle /\left(\|\mathbf{u}\|\left\|\mathbf{u}_{2}^{*}\right\|\right)$, and then multiplying the inequality on the LHS of (103) by $\left\|\alpha_{1} \mathbf{u}\right\| \cdot\left\|\alpha_{2} \mathbf{u}_{2}^{*}\right\|$ and recalling that $\left\|\alpha_{1} \mathbf{u}\right\|=\sqrt{n P_{1}}$ and that $\left\|\alpha_{2} \mathbf{u}_{2}^{*}\right\|=\sqrt{n P_{2}}$.

B) For every $\left(\mathbf{s}_{1}, \mathbf{s}_{2}, \mathcal{C}_{1}, \mathcal{C}_{2}, \mathbf{z}\right) \in \mathcal{E}_{\mathbf{X}}^{c} \cap \mathcal{E}_{\mathbf{Z}}^{c}$ and every $\mathbf{u} \in \mathcal{S}_{1}$

$$
\begin{aligned}
\left\|\mathbf{y}-\left(\alpha_{1} \mathbf{u}+\alpha_{2} \mathbf{u}_{2}^{*}\right)\right\|^{2} \leq & \left\|\mathbf{y}-\left(\alpha_{1} \mathbf{u}_{1}^{*}+\alpha_{2} \mathbf{u}_{2}^{*}\right)\right\|^{2} \\
& \Rightarrow \quad\left\langle\mathbf{y}-\alpha_{2} \mathbf{u}_{2}^{*}, \alpha_{1} \mathbf{u}\right\rangle \geq n P_{1}-n \sqrt{P_{1} N} \epsilon .
\end{aligned}
$$

Statement B) follows from rewriting the inequality on the LHS of (104) as $\|(\mathbf{y}-$ $\left.\alpha_{2} \mathbf{u}_{2}^{*}\right)-\alpha_{1} \mathbf{u}_{1}(j)\left\|^{2} \leq\right\|\left(\mathbf{y}-\alpha_{2} \mathbf{u}_{2}^{*}\right)-\alpha_{1} \mathbf{u}_{1}^{*} \|^{2}$ or equivalently as

$$
\begin{aligned}
\left\langle\mathbf{y}-\alpha_{2} \mathbf{u}_{2}^{*}, \alpha_{1} \mathbf{u}_{1}(j)\right\rangle & \geq\left\langle\mathbf{y}-\alpha_{2} \mathbf{u}_{2}^{*}, \alpha_{1} \mathbf{u}_{1}^{*}\right\rangle \\
& =\left\langle\alpha_{1} \mathbf{u}_{1}^{*}+\mathbf{z}, \alpha_{1} \mathbf{u}_{1}^{*}\right\rangle \\
& =\left\|\alpha_{1} \mathbf{u}_{1}^{*}\right\|^{2}+\left\langle\mathbf{z}, \alpha_{1} \mathbf{u}_{1}^{*}\right\rangle .
\end{aligned}
$$

It now follows from the equivalence of the first inequality in (104) with (105) that for $\left(\mathbf{s}_{1}, \mathbf{s}_{2}, \mathcal{C}_{1}, \mathcal{C}_{2}, \mathbf{z}\right) \in \mathcal{E}_{\mathbf{Z}}^{c}$, the first inequality in (104) can only hold if

$$
\left\langle\mathbf{y}-\alpha_{2} \mathbf{u}_{2}^{*}, \alpha_{1} \mathbf{u}\right\rangle \geq n P_{1}-n \sqrt{P_{1} N} \epsilon
$$

thus establishing B).

C) For every $\left(\mathbf{s}_{1}, \mathbf{s}_{2}, \mathcal{C}_{1}, \mathcal{C}_{2}, \mathbf{z}\right) \in \mathcal{E}_{\mathbf{X}}^{c} \cap \mathcal{E}_{\mathbf{Z}}^{c}$ and every $\mathbf{u} \in \mathcal{S}_{1}$,

$$
\begin{gathered}
\left(\left|\tilde{\rho}-\cos \varangle\left(\mathbf{u}, \mathbf{u}_{2}^{*}\right)\right|<7 \epsilon \text { and }\left\|\mathbf{y}-\left(\alpha_{1} \mathbf{u}+\alpha_{2} \mathbf{u}_{2}^{*}\right)\right\|^{2} \leq\left\|\mathbf{y}-\left(\alpha_{1} \mathbf{u}_{1}^{*}+\alpha_{2} \mathbf{u}_{2}^{*}\right)\right\|^{2}\right) \\
\Rightarrow \quad(107) \\
\left(\left\|\alpha_{1} \mathbf{u}-\mathbf{w}\right\|^{2} \leq n P_{1}-2\left(\zeta_{1} n\left(P_{1}-\sqrt{P_{1} N} \epsilon\right)+\zeta_{2} n \sqrt{P_{1} P_{2}}(\tilde{\rho}-7 \epsilon)\right)+\|\mathbf{w}\|^{2}\right) .
\end{gathered}
$$


Statement C) is obtained as follows:

$$
\begin{aligned}
\left\|\alpha_{1} \mathbf{u}-\mathbf{w}\right\|^{2} & =\left\|\alpha_{1} \mathbf{u}\right\|^{2}-2\left\langle\alpha_{1} \mathbf{u}, \mathbf{w}\right\rangle+\|\mathbf{w}\|^{2} \\
& =\left\|\alpha_{1} \mathbf{u}\right\|^{2}-2\left(\zeta_{1}\left\langle\alpha_{1} \mathbf{u}, \mathbf{y}-\alpha_{2} \mathbf{u}_{2}^{*}\right\rangle+\zeta_{2}\left\langle\alpha_{1} \mathbf{u}, \alpha_{2} \mathbf{u}_{2}^{*}\right\rangle\right)+\|\mathbf{w}\|^{2} \\
& \stackrel{a)}{\leq} n P_{1}-2\left(\zeta_{1} n\left(P_{1}-\sqrt{P_{1} N} \epsilon\right)+\zeta_{2} n \sqrt{P_{1} P_{2}}(\tilde{\rho}-7 \epsilon)\right)+\|\mathbf{w}\|^{2},
\end{aligned}
$$

where in $a$ ) we have used Statement A) and Statement B).

D) For every $\left(\mathbf{s}_{1}, \mathbf{s}_{2}, \mathcal{C}_{1}, \mathcal{C}_{2}, \mathbf{z}\right) \in \mathcal{E}_{\mathbf{X}}^{c} \cap \mathcal{E}_{\mathbf{Z}}^{c}$

$$
\|\mathbf{w}\|^{2} \leq n\left(\zeta_{1}^{2} P_{1}+2 \zeta_{1} \zeta_{2} \sqrt{P_{1} P_{2}} \tilde{\rho}+\zeta_{2}^{2}\left(P_{1}+N\right)+\kappa \epsilon\right),
$$

where $\kappa$ depends on $P_{1}, P_{2}, N, \zeta_{1}$ and $\zeta_{2}$ only.

Statement D) is obtained as follows

$$
\begin{aligned}
\|\mathbf{w}\|^{2} & =\zeta_{1}^{2}\left\|\alpha_{2} \mathbf{u}_{2}^{*}\right\|^{2}+2 \zeta_{1} \zeta_{2}\left\langle\alpha_{2} \mathbf{u}_{2}^{*}, \mathbf{y}-\alpha_{2} \mathbf{u}_{2}^{*}\right\rangle+\zeta_{2}^{2}\left\|\mathbf{y}-\alpha_{2} \mathbf{u}_{2}^{*}\right\|^{2} \\
& =\zeta_{1}^{2} n P_{2}+2 \zeta_{1} \zeta_{2}\left(\left\langle\alpha_{2} \mathbf{u}_{2}^{*}, \alpha_{1} \mathbf{u}_{1}^{*}\right\rangle-\left\langle\alpha_{2} \mathbf{u}_{2}^{*}, \mathbf{z}\right\rangle\right)+\zeta_{2}^{2}\left(\left\|\alpha_{1} \mathbf{u}_{1}^{*}\right\|^{2}+2\left\langle\alpha_{1} \mathbf{u}_{1}^{*}, \mathbf{z}\right\rangle+\|\mathbf{z}\|^{2}\right) \\
& \stackrel{a)}{\leq} \zeta_{1}^{2} n P_{1}+2 \zeta_{1} \zeta_{2}\left(n \sqrt{P_{1} P_{2}}(\tilde{\rho}+7 \epsilon)+n \sqrt{P_{2} N} \epsilon\right)+\zeta_{2}^{2}\left(n P_{1}+2 n \sqrt{P_{1} N} \epsilon+n N(1+\epsilon)\right) \\
& \leq n\left(\zeta_{1}^{2} P_{1}+2 \zeta_{1} \zeta_{2} \sqrt{P_{1} P_{2}} \tilde{\rho}+\zeta_{2}^{2}\left(P_{1}+N\right)+\kappa \epsilon\right),
\end{aligned}
$$

where in $a)$ we have used that $\left(\mathbf{s}_{1}, \mathbf{s}_{2}, \mathcal{C}_{1}, \mathcal{C}_{2}, \mathbf{z}\right) \in \mathcal{E}_{\mathbf{Z}}^{c}$.

E) For every $\left(\mathbf{s}_{1}, \mathbf{s}_{2}, \mathcal{C}_{1}, \mathcal{C}_{2}, \mathbf{z}\right) \in \mathcal{E}_{\mathbf{X}}^{c} \cap \mathcal{E}_{\mathbf{Z}}^{c}$ and an arbitrary $\mathbf{u} \in \mathcal{S}_{1}$,

$$
\begin{gathered}
\left(\left|\tilde{\rho}-\cos \varangle\left(\mathbf{u}, \mathbf{u}_{2}^{*}\right)\right|<7 \epsilon \quad \text { and } \quad\left\|\mathbf{y}-\left(\alpha_{1} \mathbf{u}+\alpha_{2} \mathbf{u}_{2}^{*}\right)\right\|^{2} \leq\left\|\mathbf{y}-\left(\alpha_{1} \mathbf{u}_{1}^{*}+\alpha_{2} \mathbf{u}_{2}^{*}\right)\right\|^{2}\right) \\
\Rightarrow \\
\left(\left\|\alpha_{1} \mathbf{u}-\mathbf{w}\right\|^{2} \leq \Upsilon(\epsilon)\right),
\end{gathered}
$$

where

$$
\Upsilon(\epsilon)=n \frac{P_{1} N\left(1-\tilde{\rho}^{2}\right)}{P_{1}\left(1-\tilde{\rho}^{2}\right)+N}+n \kappa^{\prime} \epsilon
$$

and where $\kappa^{\prime}$ only depends on $P, N_{1}, N_{2}, \zeta_{1}$ and $\zeta_{2}$.

Statement E) follows from combining Statement C) with Statement D) and the explicit values of $\zeta_{1}$ and $\zeta_{2}$ given in (99). 
F) For every $\mathbf{u} \in \mathcal{S}_{1}$, denote by $\varphi \in[0, \pi]$ the angle between $\mathbf{u}$ and $\mathbf{w}$, and let

$$
\mathcal{B}\left(\mathbf{s}_{1}, \mathbf{s}_{2}, \mathbf{u}_{1}^{*}, \mathbf{u}_{2}^{*}, \mathbf{z}\right) \triangleq\left\{\mathbf{u} \in \mathcal{S}_{1}^{(n)}: \cos \varphi \geq \sqrt{\frac{P_{1}\left(1-\tilde{\rho}^{2}\right)+N \tilde{\rho}^{2}}{P_{1}\left(1-\tilde{\rho}^{2}\right)+N}-\kappa^{\prime \prime} \epsilon}\right\},
$$

where $\kappa^{\prime \prime}$ only depends on $P, N_{1}, N_{2}, \zeta_{1}$ and $\zeta_{2}$, and where we assume $\epsilon$ sufficiently small such that

$$
\frac{P_{1}\left(1-\tilde{\rho}^{2}\right)+N \tilde{\rho}^{2}}{P_{1}\left(1-\tilde{\rho}^{2}\right)+N}-\kappa^{\prime \prime} \epsilon>0
$$

Then, for every $\left(\mathbf{s}_{1}, \mathbf{s}_{2}, \mathcal{C}_{1}, \mathcal{C}_{2}, \mathbf{z}\right) \in \mathcal{E}_{\mathbf{X}}^{c} \cap \mathcal{E}_{\mathbf{Z}}^{c}$,

$$
\begin{gathered}
\left(\left|\tilde{\rho}-\cos \varangle\left(\mathbf{u}, \mathbf{u}_{2}^{*}\right)\right|<7 \epsilon \text { and } \quad\left\|\mathbf{y}-\left(\alpha_{1} \mathbf{u}+\alpha_{2} \mathbf{u}_{2}^{*}\right)\right\|^{2} \leq\left\|\mathbf{y}-\left(\alpha_{1} \mathbf{u}_{1}^{*}+\alpha_{2} \mathbf{u}_{2}^{*}\right)\right\|^{2}\right) \\
\Rightarrow \quad \mathbf{u} \in \mathcal{B}\left(\mathbf{s}_{1}, \mathbf{s}_{2}, \mathbf{u}_{1}^{*}, \mathbf{u}_{2}^{*}, \mathbf{z}\right) .
\end{gathered}
$$

Statement F) follows from Statement $\mathrm{E})$ by noting that if $\mathbf{w} \neq \mathbf{0}$ and $1-\Upsilon(\epsilon) /\left(n P_{1}\right)>0$, then

$$
\left.\begin{array}{l}
\left\|\alpha_{1} \mathbf{u}\right\|^{2}=n P_{1} \\
\left\|\alpha_{1} \mathbf{u}-\mathbf{w}\right\|^{2} \leq \Upsilon(\epsilon)
\end{array}\right\} \Rightarrow \cos \varangle(\mathbf{u}, \mathbf{w}) \geq \sqrt{1-\frac{\Upsilon(\epsilon)}{n P_{1}}} .
$$

To see this, first note that for every $\alpha_{1} \mathbf{u}$, where $\mathbf{u} \in \mathcal{S}_{1}$, satisfying the condition on the LHS of (110) lies within a sphere of radius $\sqrt{\Upsilon(\epsilon)}$ centered at w. And for every $\mathbf{u} \in \mathcal{S}_{1}$ we have that $\alpha_{1} \mathbf{u}$ also lies on the centered $\mathbb{R}^{n}$-sphere of radius $\sqrt{n P_{1}}$. Hence, every $\mathbf{u} \in \mathcal{S}_{1}^{(n)}$ satisfying the condition on the LHS of (110) lies in the intersection of these two regions, which is a polar cap on the centered sphere of radius $\sqrt{n P_{1}}$. An illustration of such a polar cap is given in Figure 10. The area of this polar cap is

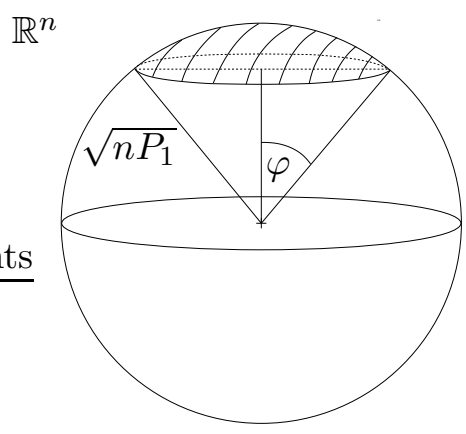

Figure 10: Polar cap of half angle $\varphi$ on an $\mathbb{R}^{n}$-sphere of radius $\sqrt{n P_{1}}$.

outer bounded as follows. Let $\mathbf{r}$ be an arbitrary point on the boundary of this polar cap. The half-angle of the polar cap would be maximized if $\mathbf{w}$ and $\mathbf{r}-\mathbf{w}$ would lie perpendicular to each other, as is illustrated in Subplot b) of Figure [11. Hence, every 


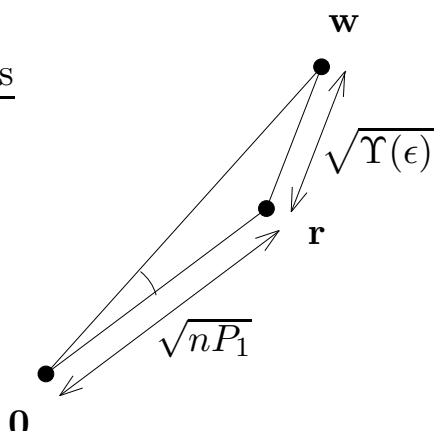

a)

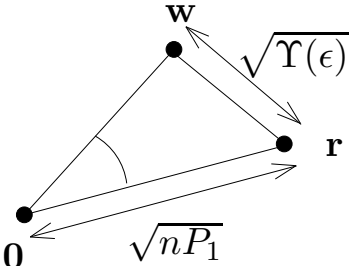

b)

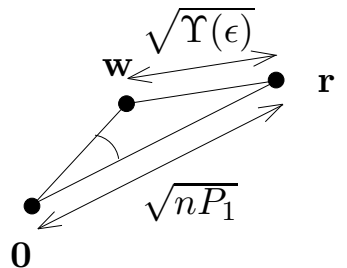

c)

Figure 11: Half-angle of cap for different constellations of $\mathbf{w}$ and $\mathbf{r}$.

$\mathbf{u} \in \mathcal{S}_{1}^{(n)}$ satisfying the upper conditions of (110) also satisfies

$$
\begin{aligned}
\cos \varphi & \geq \sqrt{1-\frac{\Upsilon(\epsilon)}{n P_{1}}} \\
& =\sqrt{\frac{P_{1}\left(1-\tilde{\rho}^{2}\right)+N \tilde{\rho}^{2}}{P_{1}\left(1-\tilde{\rho}^{2}\right)+N}-\kappa^{\prime \prime} \epsilon},
\end{aligned}
$$

where we assume $\epsilon$ sufficiently small such that $1-\Upsilon(\epsilon) /\left(n P_{1}\right)>0$ and where $\kappa^{\prime \prime}=\kappa^{\prime} / P_{1}$.

The proof of Lemma E.6 is now concluded by noticing that the set $\mathcal{E}_{\hat{\mathbf{U}}_{1}}^{\prime}$, defined in (100), is the set of tuples $\left(\mathbf{s}_{1}, \mathbf{s}_{2}, \mathcal{C}_{1}, \mathcal{C}_{2}, \mathbf{z}\right)$ for which there exists a $\mathbf{u}_{1}(j) \in \mathcal{C}_{1} \backslash\left\{\mathbf{u}_{1}^{*}\right\}$ such that $\mathbf{u}_{1}(j) \in \mathcal{B}\left(\mathbf{s}_{1}, \mathbf{s}_{2}, \mathbf{u}_{1}^{*}, \mathbf{u}_{2}^{*}, \mathbf{z}\right)$. Thus, by Statement $\left.\mathbf{F}\right)$ and by the definition of $\mathcal{E}_{\hat{\mathbf{U}}_{1}}$ in (89) it follows that

$$
\mathcal{E}_{\hat{\mathbf{U}}_{1}} \cap \mathcal{E}_{\mathbf{Z}}^{c} \cap \mathcal{E}_{\mathbf{S}}^{c} \cap \mathcal{E}_{\mathbf{X}}^{c} \subseteq \mathcal{E}_{\hat{\mathbf{U}}_{1}}^{\prime} \cap \mathcal{E}_{\mathbf{Z}}^{c} \cap \mathcal{E}_{\mathbf{S}}^{c} \cap \mathcal{E}_{\mathbf{X}}^{c}
$$

and therefore

$$
\operatorname{Pr}\left[\mathcal{E}_{\hat{\mathbf{U}}_{1}} \cap \mathcal{E}_{\mathbf{Z}}^{c} \cap \mathcal{E}_{\mathbf{S}}^{c} \cap \mathcal{E}_{\mathbf{X}}^{c}\right] \leq \operatorname{Pr}\left[\mathcal{E}_{\hat{\mathbf{U}}_{1}}^{\prime} \cap \mathcal{E}_{\mathbf{Z}}^{c} \cap \mathcal{E}_{\mathbf{S}}^{c} \cap \mathcal{E}_{\mathbf{X}}^{c}\right]
$$

We now state one more lemma that will be used for the proof of (95).

Lemma E.7. For every $\Delta \in(0,1]$, let the set $\mathcal{G}$ be given by

$$
\mathcal{G}=\left\{\left(\mathbf{s}_{1}, \mathbf{s}_{2}, \mathcal{C}_{1}, \mathcal{C}_{2}, \mathbf{z}\right): \exists \mathbf{u}_{1}(j) \in \mathcal{C}_{1} \backslash\left\{\mathbf{u}_{1}^{*}\right\} \text { s.t. } \cos \varangle\left(\mathbf{w}, \mathbf{u}_{1}(j)\right) \geq \Delta\right\},
$$

where $\mathbf{w}$ is defined in (98). Then,

$$
\left(R_{1}<-\frac{1}{2} \log _{2}\left(1-\Delta^{2}\right)\right) \Rightarrow\left(\lim _{n \rightarrow \infty} \operatorname{Pr}\left[\mathcal{G} \mid \mathcal{E}_{\mathbf{X}_{1}}^{c}\right]=0, \quad \epsilon>0\right) .
$$

Proof. The proof follows from upper bounding in every point on $\mathcal{S}_{1}$ the density of every $\mathbf{u}_{1}(j) \in \mathcal{C}_{1} \backslash\left\{\mathbf{u}_{1}^{*}\right\}$ and then using a standard argument from sphere-packing. The proof is given in Section E.5.2. 
We next state two lemmas for the proof of (97). These lemmas are similar to Lemma E.6 and Lemma E.7.

Lemma E.8. For every sufficiently small $\epsilon>0$, define the set $\mathcal{E}_{\left(\hat{\mathbf{U}}_{1}, \hat{\mathbf{U}}_{2)}\right)}^{\prime}$ as

$$
\begin{aligned}
\mathcal{E}_{\left(\hat{\mathbf{U}}_{1}, \hat{\mathbf{U}}_{2}\right)}^{\prime} \triangleq\{ & \left(\mathbf{s}_{1}, \mathbf{s}_{2}, \mathcal{C}_{1}, \mathcal{C}_{2}, \mathbf{z}\right): \exists \mathbf{u}_{1}(j) \in \mathcal{C}_{1} \backslash\left\{\mathbf{u}_{1}^{*}\right\} \quad \text { and } \quad \exists \mathbf{u}_{2}(\ell) \in \mathcal{C}_{2} \backslash\left\{\mathbf{u}_{2}^{*}\right\} \text { s.t. } \\
& \left.\cos \varangle\left(\mathbf{u}_{1}(j), \mathbf{u}_{2}(\ell)\right) \geq \tilde{\rho}-7 \epsilon \quad \text { and } \quad \cos \varangle\left(\mathbf{y}, \alpha_{1} \mathbf{u}_{1}(j)+\alpha_{2} \mathbf{u}_{2}(\ell)\right) \geq \Lambda(\epsilon)\right\},
\end{aligned}
$$

where

$$
\Lambda(\epsilon)=\sqrt{\frac{P_{1}+P_{2}+2 \tilde{\rho} \sqrt{P_{1} P_{2}}-\xi^{\prime} \epsilon}{P_{1}+P_{2}+2 \tilde{\rho} \sqrt{P_{1} P_{2}}+N+\xi_{2}} \epsilon},
$$

and where $\xi^{\prime}$ and $\xi_{2}$ depend only on $P_{1}, P_{2}$ and $N$. Then, for every sufficiently small $\epsilon>0$

$$
\mathcal{E}_{\left(\hat{\mathbf{U}}_{1}, \hat{\mathbf{U}}_{2}\right)} \cap \mathcal{E}_{\mathbf{Z}}^{c} \cap \mathcal{E}_{\mathbf{S}}^{c} \cap \mathcal{E}_{\mathbf{X}}^{c} \subseteq \mathcal{E}_{\left(\hat{\mathbf{U}}_{1}, \hat{\mathbf{U}}_{2}\right)}^{\prime} \cap \mathcal{E}_{\mathbf{Z}}^{c} \cap \mathcal{E}_{\mathbf{S}}^{c} \cap \mathcal{E}_{\mathbf{X}}^{c}
$$

and, in particular

$$
\operatorname{Pr}\left[\mathcal{E}_{\left(\hat{\mathbf{U}}_{1}, \hat{\mathbf{U}}_{2}\right)} \cap \mathcal{E}_{\mathbf{Z}}^{c} \cap \mathcal{E}_{\mathbf{S}}^{c} \cap \mathcal{E}_{\mathbf{X}}^{c}\right] \leq \operatorname{Pr}\left[\mathcal{E}_{\left(\hat{\mathbf{U}}_{1}, \hat{\mathbf{U}}_{2}\right)}^{\prime} \cap \mathcal{E}_{\mathbf{Z}}^{c} \cap \mathcal{E}_{\mathbf{S}}^{c} \cap \mathcal{E}_{\mathbf{X}}^{c}\right]
$$

Proof. We first recall that for the event $\mathcal{E}_{\left(\hat{\mathbf{U}}_{1}, \hat{\mathbf{U}}_{2}\right)}$ to occur, there must exist codewords $\mathbf{u}_{1}(j) \in \mathcal{C}_{1} \backslash\left\{\mathbf{u}_{1}^{*}\right\}$ and $\mathbf{u}_{2}(\ell) \in \mathcal{C}_{2} \backslash\left\{\mathbf{u}_{2}^{*}\right\}$ such that

$$
\left|\tilde{\rho}-\cos \varangle\left(\mathbf{u}_{1}(j), \mathbf{u}_{2}(\ell)\right)\right|<7 \epsilon,
$$

and

$$
\left\|\mathbf{y}-\left(\alpha_{1} \mathbf{u}_{1}(j)+\alpha_{2} \mathbf{u}_{2}(\ell)\right)\right\|^{2} \leq\left\|\mathbf{y}-\left(\alpha_{1} \mathbf{u}_{1}^{*}+\alpha_{2} \mathbf{u}_{2}^{*}\right)\right\|^{2} .
$$

The proof is now based on a sequence of statements related to Condition (112) and Condition (113).

A) For every $\left(\mathbf{s}_{1}, \mathbf{s}_{2}, \mathcal{C}_{1}, \mathcal{C}_{2}, \mathbf{z}\right) \in \mathcal{E}_{\mathbf{X}}^{c} \cap \mathcal{E}_{\mathbf{Z}}^{c}$,

$$
\begin{gathered}
\left\|\mathbf{y}-\left(\alpha_{1} \mathbf{u}_{1}(j)+\alpha_{2} \mathbf{u}_{2}(\ell)\right)\right\|^{2} \leq\left\|\mathbf{y}-\left(\alpha_{1} \mathbf{u}_{1}^{*}+\alpha_{2} \mathbf{u}_{2}^{*}\right)\right\|^{2} \\
\Rightarrow \\
\left\langle\mathbf{y}, \alpha_{1} \mathbf{u}_{1}(j)+\alpha_{2} \mathbf{u}_{2}(\ell)\right\rangle \geq n\left(P_{1}+P_{2}+2 \tilde{\rho} \sqrt{P_{1} P_{2}}-\xi_{1} \epsilon\right),
\end{gathered}
$$

where $\xi_{1}$ only depends on $P_{1}, P_{2}$ and $N$. 
Statement A) follows by rewriting the LHS of (114) as

$$
\begin{aligned}
2\left\langle\mathbf{y}, \alpha_{1} \mathbf{u}_{1}(j)+\alpha_{2} \mathbf{u}_{2}(\ell)\right\rangle & \geq 2\left\langle\mathbf{y}, \alpha_{1} \mathbf{u}_{1}^{*}+\alpha_{2} \mathbf{u}_{2}^{*}\right\rangle+\left\|\alpha_{1} \mathbf{u}_{1}(j)+\alpha_{2} \mathbf{u}_{2}(\ell)\right\|^{2}-\left\|\alpha_{1} \mathbf{u}_{1}^{*}+\alpha_{2} \mathbf{u}_{2}^{*}\right\|^{2} \\
& =\left\|\alpha_{1} \mathbf{u}_{1}^{*}+\alpha_{2} \mathbf{u}_{2}^{*}\right\|^{2}+2\left\langle\mathbf{z}, \alpha_{1} \mathbf{u}_{1}^{*}+\alpha_{2} \mathbf{u}_{2}^{*}\right\rangle+\left\|\alpha_{1} \mathbf{u}_{1}(j)+\alpha_{2} \mathbf{u}_{2}(\ell)\right\|^{2} \\
& \geq 2 n\left(P_{1}+2 \tilde{\rho} \sqrt{P_{1} P_{2}}(1-7 \epsilon)+P_{2}+\sqrt{P_{1} N} \epsilon+\sqrt{P_{2} N} \epsilon\right) \\
& =2 n\left(P_{1}+P_{2}+2 \tilde{\rho} \sqrt{P_{1} P_{2}}-\xi_{1} \epsilon\right)
\end{aligned}
$$

where in $a)$ we have used that $\left(\mathbf{s}_{1}, \mathbf{s}_{2}, \mathcal{C}_{1}, \mathcal{C}_{2}, \mathbf{z}\right) \in \mathcal{E}_{\mathbf{X}}^{c} \cap \mathcal{E}_{\mathbf{Z}}^{c}$ and that $\left\|\alpha_{1} \mathbf{u}_{1}(j)+\alpha_{2} \mathbf{u}_{2}(\ell)\right\|^{2} \geq 0$.

B) For every $\left(\mathbf{s}_{1}, \mathbf{s}_{2}, \mathcal{C}_{1}, \mathcal{C}_{2}, \mathbf{z}\right) \in \mathcal{E}_{\mathbf{X}}^{c} \cap \mathcal{E}_{\mathbf{Z}}^{c}$,

$$
\|\mathbf{y}\|^{2} \leq n\left(P_{1}+2 \tilde{\rho} \sqrt{P_{1} P_{2}}+P_{2}+N+\xi_{2} \epsilon\right),
$$

where $\xi_{2}$ only depends on $P_{1}, P_{2}$ and $N$.

Statement B) is obtained as follows:

$$
\begin{aligned}
\|\mathbf{y}\|^{2} & =\left\|\alpha_{1} \mathbf{u}_{1}^{*}\right\|^{2}+2\left\langle\alpha_{1} \mathbf{u}_{1}^{*}, \alpha_{2} \mathbf{u}_{2}^{*}\right\rangle+\left\|\alpha_{2} \mathbf{u}_{2}^{*}\right\|^{2}+2\left(\left\langle\alpha_{1} \mathbf{u}_{1}^{*}, \mathbf{z}\right\rangle+\left\langle\alpha_{2} \mathbf{u}_{2}^{*}, \mathbf{z}\right\rangle\right)+\|\mathbf{z}\|^{2} \\
& \stackrel{a)}{\leq} n P_{1}+2 n \tilde{\rho} \sqrt{P_{1} P_{2}}(1+7 \epsilon)+n P_{2}+2 n \sqrt{P_{1} N} \epsilon+2 n \sqrt{P_{2} N} \epsilon+n N(1+\epsilon) \\
& \leq n\left(P_{1}+2 \tilde{\rho} \sqrt{P_{1} P_{2}}+P_{2}+N+\xi_{2} \epsilon\right),
\end{aligned}
$$

where in $a)$ we have used that $\left(\mathbf{s}_{1}, \mathbf{s}_{2}, \mathcal{C}_{1}, \mathcal{C}_{2}, \mathbf{z}\right) \in \mathcal{E}_{\mathbf{X}}^{c} \cap \mathcal{E}_{\mathbf{Z}}^{c}$.

C) For every $\left(\mathbf{s}_{1}, \mathbf{s}_{2}, \mathcal{C}_{1}, \mathcal{C}_{2}, \mathbf{z}\right)$,

$$
\begin{gathered}
\left|\tilde{\rho}-\left\langle\frac{\mathbf{u}_{1}(j)}{\left\|\mathbf{u}_{1}(j)\right\|}, \frac{\mathbf{u}_{2}(\ell)}{\left\|\mathbf{u}_{2}(\ell)\right\|}\right\rangle\right|<7 \epsilon \\
\Rightarrow \\
\left\|\alpha_{1} \mathbf{u}_{1}(j)+\alpha_{2} \mathbf{u}_{2}(\ell)\right\|^{2} \leq n\left(P_{1}+2 \tilde{\rho} \sqrt{P_{1} P_{2}}+P_{2}+\xi_{3} \epsilon\right) .
\end{gathered}
$$


Statement C) follows by

$$
\begin{aligned}
\left\|\alpha_{1} \mathbf{u}_{1}(j)+\alpha_{2} \mathbf{u}_{2}(\ell)\right\|^{2} & =\left\|\alpha_{1} \mathbf{u}_{1}(j)\right\|^{2}+2\left\langle\alpha_{1} \mathbf{u}_{1}(j), \alpha_{2} \mathbf{u}_{2}(\ell)\right\rangle+\left\|\alpha_{2} \mathbf{u}_{2}(\ell)\right\|^{2} \\
& \stackrel{a)}{\leq} n P_{1}+2 n \tilde{\rho} \sqrt{P_{1} P_{2}}(1+7 \epsilon)+n P_{2} \\
& =n\left(P_{1}+2 \tilde{\rho} \sqrt{P_{1} P_{2}}+P_{2}+\xi_{3} \epsilon\right)
\end{aligned}
$$

where in $a$ ) we have used that multiplying the inequality on the LHS of (116) by $\left\|\alpha_{1} \mathbf{u}_{1}(j)\right\| \cdot\left\|\alpha_{2} \mathbf{u}_{2}(\ell)\right\|$ and recalling that $\left\|\alpha_{1} \mathbf{u}_{1}(j)\right\| \leq \sqrt{n P_{1}}$ and that $\left\|\alpha_{2} \mathbf{u}_{2}(\ell)\right\| \leq$ $\sqrt{n P_{2}}$ gives

$$
\left|n \sqrt{P_{1} P_{2}} \tilde{\rho}-\left\langle\alpha_{1} \mathbf{u}_{1}(j), \alpha_{2} \mathbf{u}_{2}(\ell)\right\rangle\right|<7 n \sqrt{P_{1} P_{2}} \epsilon
$$

and thus

$$
\left\langle\alpha_{1} \mathbf{u}_{1}(j), \alpha_{2} \mathbf{u}_{2}(\ell)\right\rangle<n \sqrt{P_{1} P_{2}} \tilde{\rho}(1+7 \epsilon),
$$

thus establishing $\mathrm{C}$ ).

D) For every $\left(\mathbf{s}_{1}, \mathbf{s}_{2}, \mathcal{C}_{1}, \mathcal{C}_{2}, \mathbf{z}\right) \in \mathcal{E}_{\mathbf{X}}^{c} \cap \mathcal{E}_{\mathbf{Z}}^{c}$,

$$
\begin{aligned}
& \left(\left|\tilde{\rho}-\cos \varangle\left(\mathbf{u}_{1}(j), \mathbf{u}_{2}(\ell)\right)\right|<7 \epsilon\right. \\
& \left.\quad \text { and } \quad\left\|\mathbf{y}-\left(\alpha_{1} \mathbf{u}_{1}(j)+\alpha_{2} \mathbf{u}_{2}(\ell)\right)\right\|^{2} \leq\left\|\mathbf{y}-\left(\alpha_{1} \mathbf{u}_{1}^{*}+\alpha_{2} \mathbf{u}_{2}^{*}\right)\right\|^{2}\right) \\
& \Rightarrow \quad \cos \varangle\left(\mathbf{y}, \alpha_{1} \mathbf{u}_{1}(j)+\alpha_{2} \mathbf{u}_{2}(\ell)\right) \geq \Lambda(\epsilon) .
\end{aligned}
$$

Statement D) follows by rewriting $\cos \varangle\left(\mathbf{y}, \alpha_{1} \mathbf{u}_{1}(j)+\alpha_{2} \mathbf{u}_{2}(\ell)\right)$ as

$$
\cos \varangle\left(\mathbf{y}, \alpha_{1} \mathbf{u}_{1}(j)+\alpha_{2} \mathbf{u}_{2}(\ell)\right)=\frac{\left\langle\mathbf{y}, \alpha_{1} \mathbf{u}_{1}(j)+\alpha_{2} \mathbf{u}_{2}(\ell)\right\rangle}{\|\mathbf{y}\| \cdot\left\|\alpha_{1} \mathbf{u}_{1}(j)+\alpha_{2} \mathbf{u}_{2}(\ell)\right\|},
$$

and then lower bounding $\left\langle\mathbf{y}, \alpha_{1} \mathbf{u}_{1}(j)+\alpha_{2} \mathbf{u}_{2}(\ell)\right\rangle$ using A) and upper bounding $\|\mathbf{y}\|$ and $\left\|\alpha_{1} \mathbf{u}_{1}(j)+\alpha_{2} \mathbf{u}_{2}(\ell)\right\|$ using $\left.\mathrm{B}\right)$ and $\mathrm{C}$ ) respectively. This, yields that for every $\left(\mathbf{s}_{1}, \mathbf{s}_{2}, \mathcal{C}_{1}, \mathcal{C}_{2}, \mathbf{z}\right) \in \mathcal{E}_{\mathbf{X}}^{c} \cap \mathcal{E}_{\mathbf{Z}}^{c}$

$$
\begin{aligned}
& \cos \varangle\left(\mathbf{y}, \alpha_{1} \mathbf{u}_{1}(j)+\alpha_{2} \mathbf{u}_{2}(\ell)\right) \\
& \geq \frac{P_{1}+P_{2}+2 \tilde{\rho} \sqrt{P_{1} P_{2}}-\xi_{1} \epsilon}{\sqrt{P_{1}+P_{2}+2 \tilde{\rho} \sqrt{P_{1} P_{2}}+N+\xi_{2}} \epsilon \sqrt{P_{1}+P_{2}+2 \tilde{\rho} \sqrt{P_{1} P_{2}}+\xi_{3} \epsilon}} \\
& \geq \sqrt{\frac{P_{1}+P_{2}+2 \tilde{\rho} \sqrt{P_{1} P_{2}}-\xi^{\prime} \epsilon}{P_{1}+P_{2}+2 \tilde{\rho} \sqrt{P_{1} P_{2}}+N+\xi_{2} \epsilon}} \\
& =\Lambda(\epsilon) \text {. }
\end{aligned}
$$


Lemma E.8 now follows by D) which gives

$$
\mathcal{E}_{\left(\hat{\mathbf{U}}_{1}, \hat{\mathbf{U}}_{2}\right)} \cap \mathcal{E}_{\mathbf{Z}}^{c} \cap \mathcal{E}_{\mathbf{S}}^{c} \cap \mathcal{E}_{\mathbf{X}}^{c} \subseteq \mathcal{E}_{\left(\hat{\mathbf{U}}_{1}, \hat{\mathbf{U}}_{2}\right)}^{\prime} \cap \mathcal{E}_{\mathbf{Z}}^{c} \cap \mathcal{E}_{\mathbf{S}}^{c} \cap \mathcal{E}_{\mathbf{X}}^{c}
$$

and therefore

$$
\operatorname{Pr}\left[\mathcal{E}_{\left(\hat{\mathbf{U}}_{1}, \hat{\mathbf{U}}_{2}\right)} \mid \mathcal{E}_{\mathbf{Z}}^{c} \cap \mathcal{E}_{\mathbf{S}}^{c} \cap \mathcal{E}_{\mathbf{X}}^{c}\right] \leq \operatorname{Pr}\left[\mathcal{E}_{\left(\hat{\mathbf{U}}_{1}, \hat{\mathbf{U}}_{2}\right)}^{\prime} \mid \mathcal{E}_{\mathbf{Z}}^{c} \cap \mathcal{E}_{\mathbf{S}}^{c} \cap \mathcal{E}_{\mathbf{X}}^{c}\right]
$$

We now state the second lemma needed for the proof of (97).

Lemma E.9. For every $\Theta \in(0,1]$ and $\Delta \in(0,1]$, let the set $\mathcal{G}$ be given by

$$
\begin{aligned}
\mathcal{G}=\left\{\left(\mathbf{s}_{1}, \mathbf{s}_{2}, \mathcal{C}_{1}, \mathcal{C}_{2}, \mathbf{z}\right):\right. & \exists \mathbf{u}_{1}(j) \in \mathcal{C}_{1} \backslash\left\{\mathbf{u}_{1}^{*}\right\}, \mathbf{u}_{2}(\ell) \in \mathcal{C}_{2} \backslash\left\{\mathbf{u}_{2}^{*}\right\} \text { s.t. } \\
& \left.\cos \varangle\left(\mathbf{u}_{1}(j), \mathbf{u}_{2}(\ell)\right) \geq \Theta, \cos \varangle\left(\mathbf{y}, \alpha_{1} \mathbf{u}_{1}(j)+\alpha_{2} \mathbf{u}_{2}(\ell)\right) \geq \Delta\right\} .
\end{aligned}
$$

Then,

$$
\begin{aligned}
&\left(R_{1}+R_{2}<-\frac{1}{2} \log _{2}(\right.\left.\left.\left(1-\Theta^{2}\right)\left(1-\Delta^{2}\right)\right)\right) \\
& \Rightarrow \quad\left(\lim _{n \rightarrow \infty} \operatorname{Pr}\left[\mathcal{G} \mid \mathcal{E}_{\mathbf{X}_{1}}^{c} \cap \mathcal{E}_{\mathbf{X}_{2}}^{c}\right]=0, \quad \epsilon>0\right) .
\end{aligned}
$$

Proof. The proof follows from upper bounding in every point on $\mathcal{S}_{i}, i \in\{1,2\}$, the density of every $\mathbf{u}_{i}(j) \in \mathcal{C}_{i} \backslash\left\{\mathbf{u}_{i}^{*}\right\}$ and then using a standard argument from spherepacking. The proof is given in Section E.5.3.

Proof of Lemma E.5. We first prove (95).

$$
\begin{aligned}
\operatorname{Pr}\left[\mathcal{E}_{\hat{\mathbf{U}}_{1}} \cap \mathcal{E}_{\mathbf{Z}}^{c} \cap \mathcal{E}_{\mathbf{S}}^{c} \cap \mathcal{E}_{\mathbf{X}}^{c}\right] & \stackrel{a)}{\leq} \operatorname{Pr}\left[\mathcal{E}_{\hat{\mathbf{U}}_{1}}^{\prime} \cap \mathcal{E}_{\mathbf{Z}}^{c} \cap \mathcal{E}_{\mathbf{S}}^{c} \cap \mathcal{E}_{\mathbf{X}}^{c}\right] \\
& \stackrel{b)}{\leq} \operatorname{Pr}\left[\mathcal{E}_{\hat{\mathbf{U}}_{1}}^{\prime} \mid \mathcal{E}_{\mathbf{X}_{1}}^{c}\right]
\end{aligned}
$$

where $a$ ) follows by Lemma E.6 and $b$ ) follows because $\mathcal{E}_{\mathbf{X}}^{c} \subseteq \mathcal{E}_{\mathbf{X}_{1}}^{c}$. The proof of (95) is now completed by combining (118) with Lemma E.7. This gives that for every $\delta>0$ and every $\epsilon>0$ there exists some $n_{41}^{\prime}(\delta, \epsilon)$ such that for all $n>n_{41}^{\prime}(\delta, \epsilon)$ we have $\operatorname{Pr}\left[\mathcal{E}_{\hat{\mathbf{U}}_{1}} \cap \mathcal{E}_{\mathbf{Z}}^{c} \cap \mathcal{E}_{\mathbf{S}}^{c} \cap \mathcal{E}_{\mathbf{X}}^{c}\right]<\delta$ whenever

$$
\begin{aligned}
R_{1} & <-\frac{1}{2} \log _{2}\left(\frac{N\left(1-\tilde{\rho}^{2}\right)}{P_{1}\left(1-\tilde{\rho}^{2}\right)+N}+\kappa^{\prime \prime} \epsilon\right) \\
& \leq \frac{1}{2} \log _{2}\left(\frac{P_{1}\left(1-\tilde{\rho}^{2}\right)+N}{N\left(1-\tilde{\rho}^{2}\right)}-\kappa_{1} \epsilon\right),
\end{aligned}
$$

where $\kappa_{1}$ is a positive constant determined by $P_{1}, P_{2}, N, \zeta_{1}$ and $\zeta_{2}$. A similar argument establishes (96).

We turn to the proof of (97).

$$
\begin{aligned}
\operatorname{Pr}\left[\mathcal{E}_{\left(\hat{\mathbf{U}}_{1}, \hat{\mathbf{U}}_{2}\right)} \cap \mathcal{E}_{\mathbf{Z}}^{c} \cap \mathcal{E}_{\mathbf{S}}^{c} \cap \mathcal{E}_{\mathbf{X}}^{c}\right] & \stackrel{a)}{\leq} \operatorname{Pr}\left[\mathcal{E}_{\left(\hat{\mathbf{U}}_{1}, \hat{\mathbf{U}}_{2}\right)}^{\prime} \cap \mathcal{E}_{\mathbf{Z}}^{c} \cap \mathcal{E}_{\mathbf{S}}^{c} \cap \mathcal{E}_{\mathbf{X}}^{c}\right] \\
& \stackrel{b)}{\leq} \operatorname{Pr}\left[\mathcal{E}_{\left(\hat{\mathbf{U}}_{1}, \hat{\mathbf{U}}_{2}\right)}^{\prime} \mid \mathcal{E}_{\mathbf{X}_{1}}^{c} \cap \mathcal{E}_{\mathbf{X}_{2}}^{c}\right]
\end{aligned}
$$


where $a$ ) follows by Lemma E.8 and $b$ ) follows because $\mathcal{E}_{\mathbf{X}}^{c} \subseteq\left(\mathcal{E}_{\mathbf{X}_{1}}^{c} \cap \mathcal{E}_{\mathbf{X}_{2}}^{c}\right)$. The proof of (97) is now completed by combining (119) with Lemma E.9, which gives that for every $\delta>0$ and every $\epsilon>0$ there exists some $n_{43}^{\prime}(\delta, \epsilon)$ such that for all $n>n_{43}^{\prime}(\delta, \epsilon)$ we have $\operatorname{Pr}\left[\mathcal{E}_{\left(\hat{\mathbf{U}}_{1}, \hat{\mathbf{U}}_{2}\right)} \cap \mathcal{E}_{\mathbf{Z}}^{c} \cap \mathcal{E}_{\mathbf{S}}^{c} \cap \mathcal{E}_{\mathbf{X}}^{c}\right]<\delta$ whenever

$$
\begin{aligned}
R_{1}+R_{2} & <\frac{1}{2} \log _{2}\left(\frac{P_{1}+P_{2}+2 \tilde{\rho} \sqrt{P_{1} P_{2}}+N+\xi_{2} \epsilon}{\left(N+\left(\xi^{\prime}+\xi_{2}\right) \epsilon\right)\left(1-\tilde{\rho}^{2}+\xi^{\prime} \epsilon\right)}\right) \\
& \leq \frac{1}{2} \log _{2}\left(\frac{P_{1}+P_{2}+2 \tilde{\rho} \sqrt{P_{1} P_{2}}+N}{N\left(1-\tilde{\rho}^{2}\right)}-\kappa_{3} \epsilon\right),
\end{aligned}
$$

where $\kappa_{3}$ is is a positive constant determined by $P_{1}, P_{2}$ and $N$.

The proof of Lemma E.1 now follows straight forwardly.

Proof of Lemma E.1. Combining (94) with Lemma E.2, Lemma E.3, Lemma E.4 and Lemma E.5, yields that for every $\delta>0$ and $0.3>\epsilon>0$ there exists some $n_{4}^{\prime}(\delta, \epsilon) \in \mathbb{N}$ such that for all $n>n_{4}^{\prime}(\delta, \epsilon)$

$$
\operatorname{Pr}\left[\mathcal{E}_{\hat{\mathbf{U}}}\right] \leq 11 \delta \quad \text { if }\left(R_{1}, R_{2}\right) \in \mathcal{R}(\epsilon) .
$$

\section{E.3.2 Concluding the Proof of Proposition E.1}

We start with four lemmas. The first lemma upper bounds the impact of atypical source outputs on the expected distortion.

Lemma E.10. For every $\epsilon>0$

$$
\frac{1}{n} \mathrm{E}\left[\left\|\mathbf{S}_{1}\right\|^{2} \mid \mathcal{E}_{\mathbf{S}}\right] \operatorname{Pr}\left[\mathcal{E}_{\mathbf{S}}\right] \leq \sigma^{2}\left(\epsilon+\operatorname{Pr}\left[\mathcal{E}_{\mathbf{S}}\right]\right) .
$$

Proof.

$$
\begin{aligned}
\frac{1}{n} \mathrm{E}\left[\left\|\mathbf{S}_{1}\right\|^{2} \mid \mathcal{E}_{\mathbf{S}}\right] \operatorname{Pr}\left[\mathcal{E}_{\mathbf{S}}\right] & =\frac{1}{n} \mathrm{E}\left[\left\|\mathbf{S}_{1}\right\|^{2}\right]-\frac{1}{n} \mathrm{E}\left[\left\|\mathbf{S}_{1}\right\|^{2} \mid \mathcal{E}_{\mathbf{S}}^{c}\right] \operatorname{Pr}\left[\mathcal{E}_{\mathbf{S}}^{c}\right] \\
& \leq \sigma^{2}-\sigma^{2}(1-\epsilon) \operatorname{Pr}\left[\mathcal{E}_{\mathbf{S}}^{c}\right] \\
& =\sigma^{2}-\sigma^{2}(1-\epsilon)\left(1-\operatorname{Pr}\left[\mathcal{E}_{\mathbf{S}}\right]\right) \\
& =\sigma^{2} \epsilon+\sigma^{2}(1-\epsilon) \operatorname{Pr}\left[\mathcal{E}_{\mathbf{S}}\right] \\
& \leq \sigma^{2}\left(\epsilon+\operatorname{Pr}\left[\mathcal{E}_{\mathbf{S}}\right]\right) .
\end{aligned}
$$

The second lemma gives upper bounds on norms related to the reconstructions $\hat{\mathbf{s}}_{1}$ and $\hat{\mathbf{s}}_{1}^{\mathrm{G}}$.

Lemma E.11. Let the reconstructions $\hat{\mathbf{s}}_{1}$ and $\hat{\mathbf{s}}_{1}^{\mathrm{G}}$ be as defined in (82) and (87). Then,

$$
\left\|\hat{\mathbf{s}}_{1}\right\|^{2} \leq 4 n \sigma^{2} \quad\left\|\hat{\mathbf{s}}_{1}^{\mathrm{G}}\right\|^{2} \leq 4 n \sigma^{2} \quad\left\|\hat{\mathbf{s}}_{1}^{\mathrm{G}}-\hat{\mathbf{s}}_{1}\right\|^{2} \leq 16 n \sigma^{2} .
$$


Proof. We start by upper bounding the squared norm of $\hat{\mathbf{s}}_{1}$

$$
\begin{aligned}
\left\|\hat{\mathbf{s}}_{1}\right\|^{2} & =\left\|\gamma_{11} \hat{\mathbf{u}}_{1}+\gamma_{12} \hat{\mathbf{u}}_{2}\right\|^{2} \\
& =\gamma_{11}^{2}\left\|\hat{\mathbf{u}}_{1}\right\|^{2}+2 \gamma_{11} \gamma_{12}\left\langle\hat{\mathbf{u}}_{1}, \hat{\mathbf{u}}_{2}\right\rangle+\gamma_{12}^{2}\left\|\hat{\mathbf{u}}_{2}\right\|^{2} \\
& \leq \gamma_{11}^{2}\left\|\hat{\mathbf{u}}_{1}\right\|^{2}+2 \gamma_{11} \gamma_{12}\left\|\hat{\mathbf{u}}_{1}\right\|\left\|\hat{\mathbf{u}}_{2}\right\|+\gamma_{12}^{2}\left\|\hat{\mathbf{u}}_{2}\right\|^{2} \\
& =\left(\gamma_{11}\left\|\hat{\mathbf{u}}_{1}\right\|+\gamma_{12}\left\|\hat{\mathbf{u}}_{2}\right\|\right)^{2} \\
& a) \\
& \leq n \sigma^{2}(1+\rho)^{2} \\
& \leq 4 n \sigma^{2}
\end{aligned}
$$

where in $a$ ) we have used (하), i.e., that $\gamma_{11}<1$ and $\gamma_{12}<\rho$, and that $\left\|\hat{\mathbf{u}}_{i}\right\| \leq \sqrt{n \sigma^{2}}$, $i \in\{1,2\}$. The upper bound on the squared norm of $\hat{\mathbf{s}}_{1}^{\mathrm{G}}$ is obtained similarly. Its proof is therefore omitted. The upper bound on the squared norm of the difference between $\hat{\mathbf{s}}_{1}$ and $\hat{\mathbf{s}}_{1}^{\mathrm{G}}$ now follows easily:

$$
\begin{aligned}
\left\|\hat{\mathbf{s}}_{1}^{\mathrm{G}}-\hat{\mathbf{s}}_{1}\right\|^{2} & \leq\left\|\hat{\mathbf{s}}_{1}^{\mathrm{G}}\right\|^{2}+2\left\|\hat{\mathbf{s}}_{1}^{\mathrm{G}}\right\|\left\|\hat{\mathbf{s}}_{1}\right\|+\left\|\hat{\mathbf{s}}_{1}\right\|^{2} \\
& =\left(\left\|\hat{\mathbf{s}}_{1}^{\mathrm{G}}\right\|+\left\|\hat{\mathbf{s}}_{1}\right\|\right)^{2} \\
& \leq 16 n \sigma^{2} .
\end{aligned}
$$

The next two lemmas are used directly in the upcoming proof of Proposition E.1. They rely on Lemma E.10 and Lemma E.11.

\section{Lemma E.12.}

$$
\frac{1}{n} \mathrm{E}\left[\left\langle\mathbf{S}_{1}, \hat{\mathbf{S}}_{1}^{\mathrm{G}}-\hat{\mathbf{S}}_{1}\right\rangle\right] \leq \sigma^{2}\left(\epsilon+17 \operatorname{Pr}\left[\mathcal{E}_{\mathbf{S}}\right]+4 \sqrt{1+\epsilon} \operatorname{Pr}\left[\mathcal{E}_{\hat{\mathbf{U}}}\right]\right) .
$$

Proof.

$$
\begin{aligned}
\frac{1}{n} \mathrm{E}\left[\left\langle\mathbf{S}_{1}, \hat{\mathbf{S}}_{1}^{\mathrm{G}}-\hat{\mathbf{S}}_{1}\right\rangle\right]= & \frac{1}{n} \mathrm{E}\left[\left\langle\mathbf{S}_{1}, \hat{\mathbf{S}}_{1}^{\mathrm{G}}-\hat{\mathbf{S}}_{1}\right\rangle \mid \mathcal{E}_{\mathbf{S}}\right] \operatorname{Pr}\left[\mathcal{E}_{\mathbf{S}}\right] \\
& +\frac{1}{n} \mathrm{E}\left[\left\langle\mathbf{S}_{1}, \hat{\mathbf{S}}_{1}^{\mathrm{G}}-\hat{\mathbf{S}}_{1}\right\rangle \mid \mathcal{E}_{\mathbf{S}}^{c} \cap \mathcal{E}_{\hat{\mathbf{U}}}\right] \operatorname{Pr}\left[\mathcal{E}_{\mathbf{S}}^{c} \cap \mathcal{E}_{\hat{\mathbf{U}}}\right] \\
& +\frac{1}{n} \underbrace{\mathrm{E}\left[\left\langle\mathbf{S}_{1}, \hat{\mathbf{S}}_{1}^{\mathrm{G}}-\hat{\mathbf{S}}_{1}\right\rangle \mid \mathcal{E}_{\mathbf{S}}^{c} \cap \mathcal{E}_{\hat{\mathbf{U}}}^{c}\right]}_{=0} \operatorname{Pr}\left[\mathcal{E}_{\mathbf{S}}^{c} \cap \mathcal{E}_{\hat{\mathbf{U}}}^{c}\right]
\end{aligned}
$$

$$
\begin{aligned}
\stackrel{a)}{\leq} & \frac{1}{n} \mathrm{E}\left[\left\|\mathbf{S}_{1}\right\|^{2}+\left\|\hat{\mathbf{S}}_{1}^{\mathrm{G}}-\hat{\mathbf{S}}_{1}\right\|^{2} \mid \mathcal{E}_{\mathbf{S}}\right] \operatorname{Pr}\left[\mathcal{E}_{\mathbf{S}}\right] \\
& +\frac{1}{n} \mathrm{E}\left[\left\|\mathbf{S}_{1}\right\|\left\|\hat{\mathbf{S}}_{1}^{\mathrm{G}}-\hat{\mathbf{S}}_{1}\right\| \mid \mathcal{E}_{\mathbf{S}}^{c} \cap \mathcal{E}_{\hat{\mathbf{U}}}\right] \operatorname{Pr}\left[\mathcal{E}_{\hat{\mathbf{U}}}\right]
\end{aligned}
$$$$
\stackrel{\text { b) }}{\leq} \frac{1}{n} \mathrm{E}\left[\left\|\mathbf{S}_{1}\right\|^{2} \mid \mathcal{E}_{\mathbf{S}}\right] \operatorname{Pr}\left[\mathcal{E}_{\mathbf{S}}\right]+16 \sigma^{2} \operatorname{Pr}\left[\mathcal{E}_{\mathbf{S}}\right]
$$$$
+\sqrt{\sigma^{2}(1+\epsilon)} \sqrt{16 \sigma^{2}} \operatorname{Pr}\left[\mathcal{E}_{\hat{\mathbf{U}}}\right]
$$

$$
\begin{aligned}
\stackrel{c)}{\leq} & \sigma^{2}\left(\epsilon+\operatorname{Pr}\left[\mathcal{E}_{\mathbf{S}}\right]\right)+16 \sigma^{2} \operatorname{Pr}\left[\mathcal{E}_{\mathbf{S}}\right] \\
& +4 \sigma^{2} \sqrt{1+\epsilon} \operatorname{Pr}\left[\mathcal{E}_{\hat{\mathbf{U}}}\right] \\
\leq & \sigma^{2}\left(\epsilon+17 \operatorname{Pr}\left[\mathcal{E}_{\mathbf{S}}\right]+4 \sqrt{1+\epsilon} \operatorname{Pr}\left[\mathcal{E}_{\hat{\mathbf{U}}}\right]\right)
\end{aligned}
$$


In the first equality the third expectation term equals zero because by $\mathcal{E}_{\hat{\mathbf{U}}}^{c}$ we have $\left\|\hat{\mathbf{s}}_{1}^{\mathrm{G}}-\hat{\mathbf{s}}_{1}\right\|=0$ and by $\mathcal{E}_{\mathbf{S}}^{c}$ the norm $\left\|\mathbf{s}_{1}\right\|$ is bounded. In $\left.a\right)$ we have used two inequalities: in the first term, the inner product is upper bounded by using that for any two vectors $\mathbf{v} \in \mathbb{R}^{n}$ and $\mathbf{w} \in \mathbb{R}^{n}$

$$
\begin{aligned}
|\langle\mathbf{v}, \mathbf{w}\rangle| & \leq\|\mathbf{v}\| \cdot\|\mathbf{w}\| \\
& \leq \frac{1}{2}\left(\|\mathbf{v}\|^{2}+\|\mathbf{w}\|^{2}\right) \\
& \leq\|\mathbf{v}\|^{2}+\|\mathbf{w}\|^{2} .
\end{aligned}
$$

The second term is upper bounded by the Cauchy-Schwarz inequality and by $\operatorname{Pr}\left[\mathcal{E}_{\mathbf{S}}^{c} \cap \mathcal{E}_{\hat{\mathbf{U}}}\right] \leq$ $\operatorname{Pr}\left[\mathcal{E}_{\hat{\mathbf{U}}}\right]$. In $b$ ) we have used Lemma E.11 and in $c$ ) we have used Lemma E.10,

\section{Lemma E.13.}

$$
\frac{1}{n} \mathrm{E}\left[\left\|\hat{\mathbf{S}}_{1}\right\|^{2}-\left\|\hat{\mathbf{S}}_{1}^{\mathrm{G}}\right\|^{2}\right] \leq 8 \sigma^{2} \operatorname{Pr}\left[\mathcal{E}_{\hat{\mathbf{U}}}\right]
$$

Proof.

$$
\begin{aligned}
\frac{1}{n} \mathrm{E}\left[\left\|\hat{\mathbf{S}}_{1}\right\|^{2}-\left\|\hat{\mathbf{S}}_{1}^{\mathrm{G}}\right\|^{2}\right]= & \frac{1}{n} \mathrm{E}\left[\left\|\hat{\mathbf{S}}_{1}\right\|^{2}-\left\|\hat{\mathbf{S}}_{1}^{\mathrm{G}}\right\|^{2} \mid \mathcal{E}_{\hat{\mathbf{U}}}\right] \operatorname{Pr}\left[\mathcal{E}_{\hat{\mathbf{U}}}\right] \\
& +\frac{1}{n} \mathrm{E}\left[\left\|\hat{\mathbf{S}}_{1}\right\|^{2}-\left\|\hat{\mathbf{S}}_{1}^{\mathrm{G}}\right\|^{2} \mid \mathcal{E}_{\hat{\mathbf{U}}}^{c}\right] \operatorname{Pr}\left[\mathcal{E}_{\hat{\mathbf{U}}}^{c}\right] \\
& \leq \frac{1}{n} \mathrm{E}\left[\left\|\hat{\mathbf{S}}_{1}\right\|^{2}+\left\|\hat{\mathbf{S}}_{1}^{\mathrm{G}}\right\|^{2} \mid \mathcal{E}_{\hat{\mathbf{U}}}\right] \operatorname{Pr}\left[\mathcal{E}_{\hat{\mathbf{U}}}\right] \\
& \stackrel{b)}{\leq} \\
& 8 \sigma^{2} \operatorname{Pr}\left[\mathcal{E}_{\hat{\mathbf{U}}}\right],
\end{aligned}
$$

where $a$ ) follows since conditional on $\mathcal{E}_{\hat{\mathbf{U}}}^{c}$ we have $\hat{\mathbf{s}}_{1}=\hat{\mathbf{s}}_{1}^{\mathrm{G}}$ and therefore $\left\|\hat{\mathbf{s}}_{1}\right\|^{2}-\left\|\hat{\mathbf{s}}_{1}^{\mathrm{G}}\right\|^{2}=$ 0 , and where $b$ ) follows by Lemma E.11.

Proof of Proposition E.1. We show that the asymptotic normalized distortion resulting from the proposed vector-quantizer scheme is the same as the asymptotic normalized distortion resulting from the genie-aided version of this scheme.

$$
\begin{aligned}
\frac{1}{n} \mathrm{E}\left[\left\|\mathbf{S}_{1}-\hat{\mathbf{S}}_{1}\right\|^{2}\right]-\frac{1}{n} \mathrm{E}[ & \left.\left\|\mathbf{S}_{1}-\hat{\mathbf{S}}_{1}^{\mathrm{G}}\right\|^{2}\right] \\
= & \frac{1}{n}\left(\mathrm{E}\left[\left\|\mathbf{S}_{1}\right\|^{2}\right]-2 \mathrm{E}\left[\left\langle\mathbf{S}_{1}, \hat{\mathbf{S}}_{1}\right\rangle\right]+\mathrm{E}\left[\left\|\hat{\mathbf{S}}_{1}\right\|^{2}\right]\right. \\
& \left.\quad-\mathrm{E}\left[\left\|\mathbf{S}_{1}\right\|^{2}\right]+2 \mathrm{E}\left[\left\langle\mathbf{S}_{1}, \hat{\mathbf{S}}_{1}^{\mathrm{G}}\right\rangle\right]-\mathrm{E}\left[\left\|\hat{\mathbf{S}}_{1}^{\mathrm{G}}\right\|^{2}\right]\right) \\
= & 2 \frac{1}{n} \mathrm{E}\left[\left\langle\mathbf{S}_{1}, \hat{\mathbf{S}}_{1}^{\mathrm{G}}-\hat{\mathbf{S}}_{1}\right\rangle\right]+\frac{1}{n} \mathrm{E}\left[\left\|\hat{\mathbf{S}}_{1}\right\|^{2}-\left\|\hat{\mathbf{S}}_{1}^{\mathrm{G}}\right\|^{2}\right] \\
\frac{a}{\leq} & 2 \sigma^{2}\left(\epsilon+17 \operatorname{Pr}\left[\mathcal{E}_{\mathbf{S}}\right]+4 \sqrt{1+\epsilon} \operatorname{Pr}\left[\mathcal{E}_{\hat{\mathbf{U}}}\right]\right) \\
& +8 \sigma^{2} \operatorname{Pr}\left[\mathcal{E}_{\hat{\mathbf{U}}}\right] \\
= & 2 \sigma^{2}\left(\epsilon+17 \operatorname{Pr}\left[\mathcal{E}_{\mathbf{S}}\right]+4(\sqrt{1+\epsilon}+1) \operatorname{Pr}\left[\mathcal{E}_{\hat{\mathbf{U}}}\right]\right)
\end{aligned}
$$


where in step $a$ ) we have used Lemma E.12 and Lemma E.13, Combining (122) with Lemma E.2 and Lemma E.1 gives that for every $\delta>0$ and $0.3>\epsilon>0$, there exists an $n^{\prime}(\delta, \epsilon)>0$ such that for all $\left(R_{1}, R_{2}\right) \in \mathcal{R}(\epsilon)$ and $n>n^{\prime}(\delta, \epsilon)$

$$
\frac{1}{n} \mathrm{E}\left[\left\|\mathbf{S}_{1}-\hat{\mathbf{S}}_{1}\right\|^{2}\right]-\frac{1}{n} \mathrm{E}\left[\left\|\mathbf{S}_{1}-\hat{\mathbf{S}}_{1}^{\mathrm{G}}\right\|^{2}\right]<2 \sigma^{2}(\epsilon+(44 \sqrt{1+\epsilon}+61) \delta) .
$$

\section{E.4 Upper Bound on Expected Distortion}

We now derive an upper bound on the achievable distortion for the proposed vectorquantizer scheme. By Corollary E.1, it suffices to analyze the genie-aided scheme. Using that $\hat{\mathbf{S}}_{1}^{\mathrm{G}}=\gamma_{11} \mathbf{U}_{1}^{*}+\gamma_{12} \mathbf{U}_{2}^{*}$, we have

$$
\begin{aligned}
\frac{1}{n} \mathrm{E}\left[\left\|\mathbf{S}_{1}-\hat{\mathbf{S}}_{1}^{\mathrm{G}}\right\|^{2}\right]=\frac{1}{n} & \left(\mathrm{E}\left[\left\|\mathbf{S}_{1}\right\|^{2}\right]-2 \gamma_{11} \mathrm{E}\left[\left\langle\mathbf{S}_{1}, \mathbf{U}_{1}^{*}\right\rangle\right]-2 \gamma_{12} \mathrm{E}\left[\left\langle\mathbf{S}_{1}, \mathbf{U}_{2}^{*}\right\rangle\right]\right. \\
& \left.+\gamma_{11}^{2} \mathrm{E}\left[\left\|\mathbf{U}_{1}^{*}\right\|^{2}\right]+2 \gamma_{11} \gamma_{12} \mathrm{E}\left[\left\langle\mathbf{U}_{1}^{*}, \mathbf{U}_{2}^{*}\right\rangle\right]+\gamma_{12}^{2} \mathrm{E}\left[\left\|\mathbf{U}_{2}^{*}\right\|^{2}\right]\right) \\
=\sigma^{2} & -2 \gamma_{11} \frac{1}{n} \mathrm{E}\left[\left\langle\mathbf{S}_{1}, \mathbf{U}_{1}^{*}\right\rangle\right]-2 \gamma_{12} \frac{1}{n} \mathrm{E}\left[\left\langle\mathbf{S}_{1}, \mathbf{U}_{2}^{*}\right\rangle\right] \\
& +\gamma_{11}^{2} \sigma^{2}\left(1-2^{-2 R_{1}}\right)+2 \gamma_{11} \gamma_{12} \frac{1}{n} \mathrm{E}\left[\left\langle\mathbf{U}_{1}^{*}, \mathbf{U}_{2}^{*}\right\rangle\right] \\
& +\gamma_{12}^{2} \sigma^{2}\left(1-2^{-2 R_{2}}\right),
\end{aligned}
$$

where in the last equality all expected squared norms have been replaced by their explicit values, i.e., $\mathrm{E}\left[\left\|\mathbf{S}_{1}\right\|^{2}\right]=n \sigma^{2}$ and $\mathrm{E}\left[\left\|\mathbf{U}_{i}\right\|^{2}\right]=n \sigma^{2}\left(1-2^{-2 R_{i}}\right)$ for $i \in\{1,2\}$. The remaining expectations of the inner products are bounded in the following three lemmas.

Lemma E.14. For every $\delta>0$ and $0.3>\epsilon>0$ and every positive integer $n$

$$
\frac{1}{n} \mathrm{E}\left[\left\langle\mathbf{S}_{1}, \mathbf{U}_{1}^{*}\right\rangle\right] \geq \sigma^{2}\left(1-2^{-2 R_{1}}\right)(1-2 \epsilon)(1-7 \delta) .
$$

Proof.

$$
\begin{aligned}
\frac{1}{n} \mathrm{E}\left[\left\langle\mathbf{S}_{1}, \mathbf{U}_{1}^{*}\right\rangle\right]= & \frac{1}{n} \underbrace{\mathrm{E}\left[\left\|\mathbf{S}_{1}\right\|\left\|\mathbf{U}_{1}^{*}\right\| \cos \varangle\left(\mathbf{S}_{1}, \mathbf{U}_{1}^{*}\right) \mid \mathcal{E}_{\mathbf{S}} \cup \mathcal{E}_{\mathbf{X}}\right]}_{\geq 0} \cdot \operatorname{Pr}\left[\mathcal{E}_{\mathbf{S}} \cup \mathcal{E}_{\mathbf{X}}\right] \\
& +\frac{1}{n} \mathrm{E}\left[\left\|\mathbf{S}_{1}\right\|\left\|\mathbf{U}_{1}^{*}\right\| \cos \varangle\left(\mathbf{S}_{1}, \mathbf{U}_{1}^{*}\right) \mid \mathcal{E}_{\mathbf{S}}^{c} \cap \mathcal{E}_{\mathbf{X}}^{c}\right] \cdot \operatorname{Pr}\left[\mathcal{E}_{\mathbf{S}}^{c} \cap \mathcal{E}_{\mathbf{X}}^{c}\right] \\
\geq & \frac{1}{n} \sqrt{n \sigma^{2}(1-\epsilon)} \sqrt{n \sigma^{2}\left(1-2^{-2 R_{1}}\right)} \sqrt{1-2^{-2 R_{1}}}(1-\epsilon) \operatorname{Pr}\left[\mathcal{E}_{\mathbf{S}}^{c} \cap \mathcal{E}_{\mathbf{X}}^{c}\right] \\
\geq & \sigma^{2}\left(1-2^{-2 R_{1}}\right)(1-\epsilon)^{2}\left(1-\operatorname{Pr}\left[\mathcal{E}_{\mathbf{S}} \cup \mathcal{E}_{\mathbf{X}}\right]\right) \\
\geq & \sigma^{2}\left(1-2^{-2 R_{1}}\right)(1-2 \epsilon)\left(1-\operatorname{Pr}\left[\mathcal{E}_{\mathbf{S}}\right]-\operatorname{Pr}\left[\mathcal{E}_{\mathbf{X}}\right]\right),
\end{aligned}
$$

where in the first equality the first expectation term is non-negative because conditioned on $\mathcal{E}_{\mathbf{X}}$ either $\mathbf{U}_{1}^{*}=\mathbf{0}$ or, if $\mathbf{U}_{1}^{*} \neq \mathbf{0}$, then $\cos \left(\varangle\left(\mathbf{S}_{1}, \mathbf{U}_{1}^{*}\right)\right)>0$.

By Lemma E.2 and Lemma E.4 it now follows that for every $\delta>0$ and $0.3>\epsilon>0$ there exists an $n^{\prime}(\delta, \epsilon) \in \mathbb{N}$ such that for all $n>n^{\prime}(\delta, \epsilon)$

$$
\frac{1}{n} \mathrm{E}\left[\left\langle\mathbf{S}_{1}, \mathbf{U}_{1}^{*}\right\rangle\right] \geq \sigma^{2}\left(1-2^{-2 R_{1}}\right)(1-2 \epsilon)(1-7 \delta) .
$$


Lemma E.15. For every $\delta>0$ and $0.3>\epsilon>0$, there exists an $n_{2}^{\prime}(\delta, \epsilon) \in \mathbb{N}$ such that for all $n>n_{2}^{\prime}(\delta, \epsilon)$

Proof.

$$
\frac{1}{n} \mathrm{E}\left[\left\langle\mathbf{U}_{1}^{*}, \mathbf{U}_{2}^{*}\right\rangle\right] \leq \sigma^{2} 6 \delta+\sigma^{2} \rho\left(1-2^{-2 R_{1}}\right)\left(1-2^{-2 R_{2}}\right)(1+7 \epsilon) .
$$

$$
\begin{aligned}
\frac{1}{n} \mathrm{E}\left[\left\langle\mathbf{U}_{1}^{*}, \mathbf{U}_{2}^{*}\right\rangle\right]= & \frac{1}{n} \mathrm{E}\left[\left\langle\mathbf{U}_{1}^{*}, \mathbf{U}_{2}^{*}\right\rangle \mid \mathcal{E}_{\mathbf{X}}\right] \operatorname{Pr}\left[\mathcal{E}_{\mathbf{X}}\right]+\frac{1}{n} \mathrm{E}\left[\left\langle\mathbf{U}_{1}^{*}, \mathbf{U}_{2}^{*}\right\rangle \mid \mathcal{E}_{\mathbf{X}}^{c}\right] \operatorname{Pr}\left[\mathcal{E}_{\mathbf{X}}^{c}\right] \\
\leq & \frac{1}{n} \mathrm{E}\left[\left\|\mathbf{U}_{1}^{*}\right\|\left\|\mathbf{U}_{2}^{*}\right\| \mid \mathcal{E}_{\mathbf{X}}\right] \operatorname{Pr}\left[\mathcal{E}_{\mathbf{X}}\right]+\frac{1}{n} \mathrm{E}\left[\left\langle\mathbf{U}_{1}^{*}, \mathbf{U}_{2}^{*}\right\rangle \mid \mathcal{E}_{\mathbf{X}}^{c}\right] \\
\leq & \sigma^{2} \sqrt{\left(1-2^{-2 R_{1}}\right)\left(1-2^{-2 R_{2}}\right)} \operatorname{Pr}\left[\mathcal{E}_{\mathbf{X}}\right] \\
& +\frac{1}{n} \mathrm{E}\left[\tilde{\rho}(1+7 \epsilon) \sqrt{n \sigma^{2}\left(1-2^{-2 R_{1}}\right)} \sqrt{n \sigma^{2}\left(1-2^{-2 R_{2}}\right)} \mid \mathcal{E}_{\mathbf{X}}^{c}\right] \\
\leq & \sigma^{2} \operatorname{Pr}\left[\mathcal{E}_{\mathbf{X}}\right]+\sigma^{2} \rho\left(1-2^{-2 R_{1}}\right)\left(1-2^{-2 R_{2}}\right)(1+7 \epsilon) .
\end{aligned}
$$

Thus, it follows by Lemma E.4 that for every $\delta>0$ and $0.3>\epsilon>0$ there exists an $n_{2}^{\prime}(\delta, \epsilon) \in \mathbb{N}$ such that for all $n>n_{2}^{\prime}(\delta, \epsilon)$

$$
\mathrm{E}\left[\left\langle\mathbf{U}_{1}^{*}, \mathbf{U}_{2}^{*}\right\rangle\right] \leq \sigma^{2} 6 \delta+\sigma^{2} \rho\left(1-2^{-2 R_{1}}\right)\left(1-2^{-2 R_{2}}\right)(1+7 \epsilon)
$$

Lemma E.16. For every $\delta>0$ and $0.3>\epsilon>0$, there exists an $n^{\prime}(\delta, \epsilon) \in \mathbb{N}$ such that for all $n>n^{\prime}(\delta, \epsilon)$

$$
\frac{1}{n} \mathrm{E}\left[\left\langle\mathbf{S}_{1}, \mathbf{U}_{2}^{*}\right\rangle\right] \geq \sigma^{2} \rho\left(1-2^{-2 R_{2}}\right)(1-\epsilon)^{3}-\sigma^{2}(\epsilon+21 \delta+6 \delta \epsilon) .
$$

Proof. We begin with the following decomposition:

$$
\begin{aligned}
\frac{1}{n} \mathrm{E}\left[\left\langle\mathbf{S}_{1}, \mathbf{U}_{2}^{*}\right\rangle\right]= & \frac{1}{n} \mathrm{E}\left[\left\langle\mathbf{S}_{1}, \mathbf{U}_{2}^{*}\right\rangle \mid \mathcal{E}_{\mathbf{S}} \cup \mathcal{E}_{\mathbf{X}_{2}}\right] \operatorname{Pr}\left[\mathcal{E}_{\mathbf{S}} \cup \mathcal{E}_{\mathbf{X}_{2}}\right] \\
& +\frac{1}{n} \mathrm{E}\left[\left\langle\mathbf{S}_{1}, \mathbf{U}_{2}^{*}\right\rangle \mid \mathcal{E}_{\mathbf{S}}^{c} \cap \mathcal{E}_{\mathbf{X}_{2}}^{c}\right] \operatorname{Pr}\left[\mathcal{E}_{\mathbf{S}}^{c} \cap \mathcal{E}_{\mathbf{X}_{2}}^{c}\right] .
\end{aligned}
$$

The first term on the RHS of (125) is lower bounded as follows.

$$
\begin{aligned}
& \frac{1}{n} \mathrm{E}\left[\left\langle\mathbf{S}_{1}, \mathbf{U}_{2}^{*}\right\rangle \mid \mathcal{E}_{\mathbf{S}} \cup \mathcal{E}_{\mathbf{X}_{2}}\right] \operatorname{Pr}\left[\mathcal{E}_{\mathbf{S}} \cup \mathcal{E}_{\mathbf{X}_{2}}\right] \\
& \geq-\frac{1}{n} \mathrm{E}\left[\left\|\mathbf{S}_{1}\right\|^{2}+\left\|\mathbf{U}_{2}^{*}\right\|^{2} \mid \mathcal{E}_{\mathbf{S}} \cup \mathcal{E}_{\mathbf{X}_{2}}\right] \operatorname{Pr}\left[\mathcal{E}_{\mathbf{S}} \cup \mathcal{E}_{\mathbf{X}_{2}}\right] \\
& \geq-\frac{1}{n}\left(\mathrm{E}\left[\left\|\mathbf{S}_{1}\right\|^{2} \mid \mathcal{E}_{\mathbf{S}}\right] \operatorname{Pr}\left[\mathcal{E}_{\mathbf{S}}\right]\right. \\
&+\mathrm{E}\left[\left\|\mathbf{S}_{1}\right\|^{2} \mid \mathcal{E}_{\mathbf{S}}^{c} \cap \mathcal{E}_{\mathbf{X}_{2}}\right] \operatorname{Pr}\left[\mathcal{E}_{\mathbf{S}}^{c} \cap \mathcal{E}_{\mathbf{X}}\right] \\
&\left.+\left\|\mathbf{U}_{2}^{*}\right\|^{2}\left(\operatorname{Pr}\left[\mathcal{E}_{\mathbf{S}}\right]+\operatorname{Pr}\left[\mathcal{E}_{\mathbf{X}}\right]\right)\right) \\
& \geq\left(\sigma^{2}\left(\epsilon+\operatorname{Pr}\left[\mathcal{E}_{\mathbf{S}}\right]\right)+\sigma^{2}(1+\epsilon) \operatorname{Pr}\left[\mathcal{E}_{\mathbf{X}}\right]\right. \\
&\left.+\sigma^{2}\left(1-2^{-2 R_{2}}\right)\left(\operatorname{Pr}\left[\mathcal{E}_{\mathbf{S}}\right]+\operatorname{Pr}\left[\mathcal{E}_{\mathbf{X}}\right]\right)\right) \\
& \geq-\sigma^{2}\left(\epsilon+2 \operatorname{Pr}\left[\mathcal{E}_{\mathbf{S}}\right]+(2+\epsilon) \operatorname{Pr}\left[\mathcal{E}_{\mathbf{X}}\right]\right)
\end{aligned}
$$


where in $a$ ) we have used (121), in $b$ ) we have used that $\mathcal{E}_{\mathbf{X}} \supseteq \mathcal{E}_{\mathbf{X}_{2}}$, and in $c$ ) we have used Lemma E.10.

We now turn to lower bounding the second term on the RHS of (125). The probability term is lower bounded as follows

$$
\begin{aligned}
\operatorname{Pr}\left[\mathcal{E}_{\mathbf{S}}^{c} \cap \mathcal{E}_{\mathbf{X}_{2}}^{c}\right] & =1-\operatorname{Pr}\left[\mathcal{E}_{\mathbf{S}} \cup \mathcal{E}_{\mathbf{X}_{2}}\right] \\
& \geq 1-\left(\operatorname{Pr}\left[\mathcal{E}_{\mathbf{S}}\right]+\operatorname{Pr}\left[\mathcal{E}_{\mathbf{X}}\right]\right) .
\end{aligned}
$$

To lower bound the expectation term, we represent $\mathbf{u}_{i}^{*}$ as a scaled version of $\mathbf{s}_{i}$ corrupted by an additive "quantization noise" $\mathbf{v}_{i}$. More precisely,

$$
\mathbf{u}_{i}^{*}=\nu_{i} \mathbf{s}_{i}+\mathbf{v}_{i} \quad \text { where } \quad \nu_{i}=\frac{\left\|\mathbf{u}_{i}^{*}\right\|}{\left\|\mathbf{s}_{i}\right\|} \cos \varangle\left(\mathbf{s}_{i}, \mathbf{u}_{i}^{*}\right) \quad i \in\{1,2\} .
$$

With this choice of $\nu_{i}$, the vector $\mathbf{v}_{i}$ is always orthogonal to $\mathbf{s}_{i}$. By (128), the inner product $\left\langle\mathbf{S}_{1}, \mathbf{U}_{2}^{*}\right\rangle$ can now be rewritten as $\nu_{2}\left\langle\mathbf{S}_{1}, \mathbf{S}_{2}\right\rangle+\left\langle\mathbf{S}_{1}, \mathbf{V}_{2}\right\rangle$. Hence,

$$
\begin{aligned}
& \mathrm{E}\left[\left\langle\mathbf{S}_{1}, \mathbf{U}_{2}^{*}\right\rangle \mid \mathcal{E}_{\mathbf{S}}^{c} \cap \mathcal{E}_{\mathbf{X}_{2}}^{c}\right] \\
& \stackrel{a)}{=} \mathrm{E}_{\mathbf{S}_{1}, \mathbf{S}_{2}}\left[\mathrm{E}_{\mathscr{C}_{1}, \mathscr{C}_{2}}\left[\nu_{2}\left\langle\mathbf{s}_{1}, \mathbf{s}_{2}\right\rangle \mid\left(\mathbf{S}_{1}, \mathbf{S}_{2}\right)=\left(\mathbf{s}_{1}, \mathbf{s}_{2}\right), \mathcal{E}_{\mathbf{S}}^{c} \cap \mathcal{E}_{\mathbf{X}_{2}}^{c}\right]\right. \\
& +\underbrace{\mathrm{E}_{\mathscr{C}_{1}, \mathscr{C}_{2}}\left[\left\langle\mathbf{s}_{1}, \mathbf{V}_{2}\right\rangle \mid\left(\mathbf{S}_{1}, \mathbf{S}_{2}\right)=\left(\mathbf{s}_{1}, \mathbf{s}_{2}\right), \mathcal{E}_{\mathbf{S}}^{c} \cap \mathcal{E}_{\mathbf{X}_{2}}^{c}\right]}_{=0}] \\
& =\mathrm{E}_{\mathbf{S}_{1}, \mathbf{S}_{2}}\left[\frac{\left\|\mathbf{U}_{2}^{*}\right\|}{\left\|\mathbf{S}_{2}\right\|}\left\langle\mathbf{S}_{1}, \mathbf{S}_{2}\right\rangle \mathrm{E}_{\mathscr{C}_{1}, \mathscr{C}_{2}}\left[\cos \varangle\left(\mathbf{s}_{2}, \mathbf{U}_{2}^{*}\right) \mid\left(\mathbf{S}_{1}, \mathbf{S}_{2}\right)=\left(\mathbf{s}_{1}, \mathbf{s}_{2}\right), \mathcal{E}_{\mathbf{S}}^{c} \cap \mathcal{E}_{\mathbf{X}_{2}}^{c}\right]\right] \\
& \stackrel{b)}{\geq} \mathrm{E}_{\mathbf{S}_{1}, \mathbf{S}_{2}}\left[\left\|\mathbf{U}_{2}^{*}\right\|\left\|\mathbf{S}_{1}\right\| \cos \left(\varangle\left(\mathbf{S}_{1}, \mathbf{S}_{2}\right)\right) \sqrt{1-2^{-2 R_{2}}}(1-\epsilon) \mid \mathcal{E}_{\mathbf{S}}^{c} \cap \mathcal{E}_{\mathbf{X}_{2}}^{c}\right] \\
& \stackrel{c)}{\geq n \sigma^{2}\left(1-2^{-2 R_{2}}\right)} \sqrt{n \sigma^{2}(1-\epsilon)} \rho(1-\epsilon) \sqrt{1-2^{-2 R_{2}}}(1-\epsilon) \\
& \geq n \rho \sigma^{2}\left(1-2^{-2 R_{2}}\right)(1-\epsilon)^{3},
\end{aligned}
$$

where we have denoted by $\mathscr{C}_{i}$ the random codebook of user $i \in\{1,2\}$, and where in $a$ ) the second expectation term is zero because for every $\left(\mathbf{s}_{1}, \mathbf{s}_{2}\right) \in \mathcal{E}_{\mathbf{S}}^{c}$

$$
\mathrm{E}_{\mathscr{C}_{2}}\left[\left\langle\mathbf{s}_{1}, \mathbf{V}_{2}\right\rangle \mid\left(\mathbf{S}_{1}, \mathbf{S}_{2}\right)=\left(\mathbf{s}_{1}, \mathbf{s}_{2}\right), \mathcal{E}_{\mathbf{X}_{2}}^{c}\right]=0
$$

This holds since in the expectation over the codebooks $\mathscr{C}_{2}$ with conditioning on $\mathcal{E}_{\mathbf{X}_{2}}^{c}$, for every $\mathbf{v}_{2} \in \mathbb{R}^{n}$ the sequences $\mathbf{v}_{2}$ and $-\mathbf{v}_{2}$ are equiprobable and thus their inner products with $\mathbf{s}_{1}$ cancel off each other. Inequality $b$ ) follows from lower bounding $\cos \varangle\left(\mathbf{s}_{2}, \mathbf{U}_{2}^{*}\right)$ conditioned on $\mathcal{E}_{\mathbf{X}}^{c}$ combined with the fact that conditioned on $\mathcal{E}_{\mathbf{S}}^{c}$ the term $\cos \varangle\left(\mathbf{S}_{1}, \mathbf{S}_{2}\right)$ is positive. Inequality c) follows from lower bounding $\left\|\mathbf{S}_{1}\right\|$ and $\cos \varangle\left(\mathbf{S}_{1}, \mathbf{S}_{2}\right)$ conditioned on $\mathcal{E}_{\mathbf{S}}^{c}$. 
Combining (125) with (126), (127) and (129) gives

$$
\begin{aligned}
\frac{1}{n} \mathrm{E}\left[\left\langle\mathbf{S}_{1}, \mathbf{U}_{2}^{*}\right\rangle\right] \geq & -\sigma^{2}\left(\epsilon+2 \operatorname{Pr}\left[\mathcal{E}_{\mathbf{S}}\right]+(2+\epsilon) \operatorname{Pr}\left[\mathcal{E}_{\mathbf{X}}\right]\right) \\
& +\sigma^{2} \rho\left(1-2^{-2 R_{2}}\right)(1-\epsilon)^{3}\left(1-\left(\operatorname{Pr}\left[\mathcal{E}_{\mathbf{S}}\right]+\operatorname{Pr}\left[\mathcal{E}_{\mathbf{X}}\right]\right)\right) \\
\geq & \sigma^{2} \rho\left(1-2^{-2 R_{2}}\right)(1-\epsilon)^{3}-\sigma^{2}\left(\epsilon+3 \operatorname{Pr}\left[\mathcal{E}_{\mathbf{S}}\right]+(3+\epsilon) \operatorname{Pr}\left[\mathcal{E}_{\mathbf{X}}\right]\right) .
\end{aligned}
$$

Thus, by Lemma E.2 and Lemma E.4 it follows that for every $\delta>0$ and $0.3>\epsilon>0$ there exists an $n^{\prime}(\delta, \epsilon) \in \mathbb{N}$ such that for all $n>n^{\prime}(\delta, \epsilon)$

$$
\frac{1}{n} \mathrm{E}\left[\left\langle\mathbf{S}_{1}, \mathbf{U}_{2}^{*}\right\rangle\right] \geq \sigma^{2} \rho\left(1-2^{-2 R_{2}}\right)(1-\epsilon)^{3}-\sigma^{2}(\epsilon+21 \delta+6 \delta \epsilon) .
$$

The distortion of the genie-aided scheme is now upper bounded as follows

$$
\begin{aligned}
\frac{1}{n} \mathrm{E}\left[\left\|\mathbf{S}_{1}-\hat{\mathbf{S}}_{1}^{\mathrm{G}}\right\|^{2}\right]= & \sigma^{2}-2 \gamma_{11} \frac{1}{n} \mathrm{E}\left[\left\langle\mathbf{S}_{1}, \mathbf{U}_{1}^{*}\right\rangle\right]-2 \gamma_{12} \frac{1}{n} \mathrm{E}\left[\left\langle\mathbf{S}_{1}, \mathbf{U}_{2}^{*}\right\rangle\right] \\
& +\gamma_{11}^{2} \sigma^{2}\left(1-2^{-2 R_{1}}\right)+2 \gamma_{11} \gamma_{12} \frac{1}{n} \mathrm{E}\left[\left\langle\mathbf{U}_{1}^{*}, \mathbf{U}_{2}^{*}\right\rangle\right] \\
& +\gamma_{12}^{2} \sigma^{2}\left(1-2^{-2 R_{2}}\right) \\
\stackrel{a)}{\leq} & \sigma^{2} 2^{-2 R_{1}} \frac{1-\rho^{2}\left(1-2^{-2 R_{2}}\right)}{1-\tilde{\rho}^{2}}+\xi^{\prime}(\delta, \epsilon),
\end{aligned}
$$

where in $a$ ) we have used Lemma E.14, Lemma E.15 and Lemma E.16, and where

$$
\lim _{\delta, \epsilon \rightarrow 0} \xi^{\prime}(\delta, \epsilon)=0
$$

\section{E.5 Proofs of Lemma E.4, Lemma E.7 and Lemma E.9}

The proofs in this section rely on bounds from the geometry of sphere packing. To this end, we denote by $C_{n}(\varphi)$ the surface area of a polar cap of half angle $\varphi$ on an $\mathbb{R}^{n}$-sphere of unit radius. An illustration of $C_{n}(\varphi)$ is given in Figure 12, Upper and lower bounds

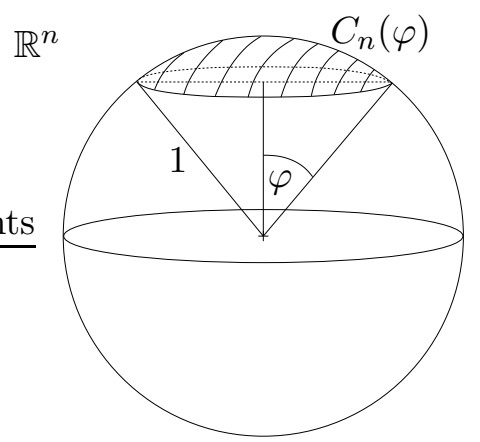

Figure 12: Polar cap of half angle $\varphi$.

on the surface area $C_{n}(\varphi)$ are given in the following lemma.

Lemma E.17. For any $\varphi \in[0, \pi / 2]$,

$$
\frac{\Gamma\left(\frac{n}{2}+1\right) \sin ^{(n-1)} \varphi}{n \Gamma\left(\frac{n+1}{2}\right) \sqrt{\pi} \cos \varphi}\left(1-\frac{1}{n} \tan ^{2} \varphi\right) \leq \frac{C_{n}(\varphi)}{C_{n}(\pi)} \leq \frac{\Gamma\left(\frac{n}{2}+1\right) \sin ^{(n-1)} \varphi}{n \Gamma\left(\frac{n+1}{2}\right) \sqrt{\pi} \cos \varphi} .
$$


Proof. See [23, Inequality (27)].

The ratio of the two gamma functions that appears in the upper bound and the lower bound of Lemma E.17 has the following asymptotic series.

Lemma E.18.

$$
\frac{\Gamma\left(x+\frac{1}{2}\right)}{\Gamma(x)}=\sqrt{x}\left(1-\frac{1}{8 x}+\frac{1}{128 x^{2}}+\frac{5}{1024 x^{3}}-\frac{21}{32768 x^{4}}+\ldots\right)
$$

and in particular

$$
\lim _{x \rightarrow \infty} \frac{\Gamma\left(x+\frac{1}{2}\right)}{\Gamma(x) \sqrt{x}}=1
$$

Proof. We first note that

$$
\begin{aligned}
\frac{\Gamma\left(x+\frac{1}{2}\right)}{\Gamma(x)} & =\frac{(2 x-1) ! !}{2^{x}(x-1) !} \sqrt{\pi} \\
& =\frac{x}{4^{x}}\left(\begin{array}{c}
2 x \\
x
\end{array}\right) \sqrt{\pi},
\end{aligned}
$$

where $\xi !$ ! denotes the double factorial of $\xi$. The proof now follows by combining (130) with

$$
\left(\begin{array}{c}
2 x \\
x
\end{array}\right)=\frac{4^{x}}{\sqrt{\pi x}}\left(1-\frac{1}{8 x}+\frac{1}{128 x^{2}}+\frac{5}{1024 x^{3}}-\frac{21}{32768 x^{4}}+\ldots\right),
$$

which is given in [24, Problem 9.60, p. 495].

Before starting with the proofs of this section, we give one more lemma. To this end, whenever the vector-quantizer of Encoder 1 does not produce the all-zero sequence, denote by $\varsigma_{1}\left(\mathbf{s}_{1}, \mathcal{C}_{1}\right)$ the index of $\mathbf{u}_{1}^{*}$ in its codebook $\mathcal{C}_{1}$. And whenever the vector-quantizer of Encoder 1 produces the all-zero sequence, let $\varsigma_{1}\left(\mathbf{s}_{1}, \mathcal{C}_{1}\right)=0$. Further, let $\lambda_{1}(\cdot)$ denote the measure on the codeword sphere $\mathcal{S}_{1}$ induced by the uniform distribution, and let $f^{\lambda_{1}}(\cdot)$ denote the density on $\mathcal{S}_{1}$ with respect to $\lambda_{1}(\cdot)$. Similarly, for Encoder 2 define $\varsigma_{2}\left(\mathbf{s}_{2}, \mathcal{C}_{2}\right)$ and $f^{\lambda_{2}}(\cdot)$.

Lemma E.19. Conditional on $\varsigma_{1}\left(\mathbf{S}_{1}, \mathscr{C}_{1}\right)=1$, the density of $\mathbf{U}_{1}(j)$ is upper bounded for every $j \in\left\{2,3, \ldots, 2^{n R_{1}}\right\}$ and at every point $\mathbf{u} \in \mathcal{S}_{1}$ by twice the uniform density:

$$
f^{\lambda_{1}}\left(\mathbf{U}_{1}(j)=\mathbf{u} \mid \varsigma_{1}\left(\mathbf{S}_{1}, \mathscr{C}_{1}\right)=1\right) \leq 2 \cdot \frac{1}{r_{1}^{n-1} C_{n}(\pi)}, \quad \mathbf{u} \in \mathcal{S}_{1}, j \in\left\{2,3, \ldots, 2^{n R_{1}}\right\} .
$$

And similarly for Encoder 2.

Proof. We first write the conditional density as an average over $\cos \varangle\left(\mathbf{S}_{1}, \mathbf{U}_{1}(1)\right)$. Since conditioned on $\varsigma_{1}\left(\mathbf{S}_{1}, \mathscr{C}_{1}\right)=1$ we have $\cos \varangle\left(\mathbf{S}_{1}, \mathbf{U}_{1}(1)\right) \in\left[\sqrt{1-2^{-2 R_{1}}}(1-\epsilon), \sqrt{1-2^{-2 R_{1}}}(1+\right.$ $\epsilon)$ ], we obtain

$$
\begin{aligned}
& f^{\lambda_{1}}\left(\mathbf{U}_{1}(j)=\mathbf{u} \mid \varsigma_{1}\left(\mathbf{S}_{1}, \mathscr{C}_{1}\right)=1\right) \\
& =\int_{\mathbf{s}_{1} \in \mathbb{R}^{n}} \int_{\sqrt{1-2^{-2 R_{1}}}(1-\epsilon)}^{\sqrt{1-2^{-2 R_{1}}}(1+\epsilon)} f^{\lambda_{1}}\left(\mathbf{U}_{1}(j)=\mathbf{u} \mid \mathbf{S}_{1}=\mathbf{s}_{1}, \varsigma_{1}\left(\mathbf{s}_{1}, \mathscr{C}_{1}\right)=1, \cos \varangle\left(\mathbf{s}_{1}, \mathbf{U}_{1}(1)\right)=a\right) \\
& f\left(\mathbf{S}_{1}=\mathbf{s}_{1}, \cos \varangle\left(\mathbf{s}_{1}, \mathbf{U}_{1}(1)\right)=a \mid \varsigma_{1}\left(\mathbf{S}_{1}, \mathscr{C}_{1}\right)=1\right) \operatorname{ạ} \mathbf{s}_{1} . \quad
\end{aligned}
$$


The proof now follows by upper bounding the conditional density

$$
f^{\lambda_{1}}\left(\mathbf{U}_{1}(j)=\mathbf{u} \mid \mathbf{S}_{1}=\mathbf{s}_{1}, \varsigma_{1}\left(\mathbf{s}_{1}, \mathscr{C}_{1}\right)=1, \cos \varangle\left(\mathbf{s}_{1}, \mathbf{U}_{1}(1)\right)=a\right) .
$$

To this end, define for every $a \in\left[\sqrt{1-2^{-2 R_{1}}}(1-\epsilon), \sqrt{1-2^{-2 R_{1}}}(1+\epsilon)\right]$

$$
\mathscr{D}_{a}\left(\mathbf{s}_{1}\right) \triangleq\left\{\mathbf{u} \in \mathcal{S}_{1}:\left|\cos \varangle\left(\mathbf{s}_{1}, \mathbf{u}\right)-\sqrt{1-2^{-2 R_{1}}}\right| \leq\left|a-\sqrt{1-2^{-2 R_{1}}}\right|\right\},
$$

and

$$
\mathscr{D}_{a}^{c}\left(\mathbf{s}_{1}\right) \triangleq\left\{\mathbf{u} \in \mathcal{S}_{1}:\left|\cos \varangle\left(\mathbf{s}_{1}, \mathbf{u}\right)-\sqrt{1-2^{-2 R_{1}}}\right|>\left|a-\sqrt{1-2^{-2 R_{1}}}\right|\right\} .
$$

The conditional density can now be upper bounded by distinguishing between $\mathbf{u} \in$ $\mathscr{D}_{a}\left(\mathbf{s}_{1}\right)$ and $\mathbf{u} \in \mathscr{D}_{a}^{c}\left(\mathbf{s}_{1}\right)$. If $\mathbf{u} \in \mathscr{D}_{a}\left(\mathbf{s}_{1}\right)$, then the conditional density is zero because the fact that $\varsigma_{1}\left(\mathbf{s}_{1}, \mathscr{C}_{1}\right)$ is 1 implies that

$$
\left|\cos \varangle\left(\mathbf{s}_{1}, \mathbf{U}_{1}(j)\right)-\sqrt{1-2^{-2 R_{1}}}\right|>\left|a-\sqrt{1-2^{-2 R_{1}}}\right|, \quad \forall j \in\left\{2,3, \ldots, 2^{n R_{1}}\right\} .
$$

And if $\mathbf{u} \in \mathscr{D}_{a}^{c}\left(\mathbf{s}_{1}\right)$ the conditional density is uniform over $\mathscr{D}_{a}^{c}\left(\mathbf{s}_{1}\right)$, i.e.

$$
f^{\lambda_{1}}\left(\mathbf{U}_{1}(j)=\mathbf{u} \mid \mathbf{S}_{1}=\mathbf{s}_{1}, \varsigma_{1}\left(\mathbf{s}_{1}, \mathscr{C}_{1}\right)=1, \cos \varangle\left(\mathbf{s}_{1}, \mathbf{U}_{1}(1)\right)=a\right)=v, \quad \mathbf{u} \in \mathscr{D}_{a}^{c}\left(\mathbf{s}_{1}\right),
$$

for some $v>0$. Thus,

$$
\begin{aligned}
& f^{\lambda_{1}}\left(\mathbf{U}_{1}(j)=\mathbf{u} \mid \mathbf{S}_{1}=\mathbf{s}_{1}, \varsigma_{1}\left(\mathbf{s}_{1}, \mathscr{C}_{1}\right)=1, \cos \varangle\left(\mathbf{s}_{1}, \mathbf{U}_{1}(1)\right)=a\right) \leq v, \\
& \forall \mathbf{u} \in \mathcal{S}_{1}, \mathbf{s}_{1} \in \mathbb{R}^{n}, a \in\left[\sqrt{1-2^{-2 R_{1}}}(1-\epsilon), \sqrt{1-2^{-2 R_{1}}}(1+\epsilon)\right] .
\end{aligned}
$$

It now remains to upper bound $v$. To this end, notice that the surface area of $\mathscr{D}_{a}\left(\mathbf{s}_{1}\right)$ never exceeds half the surface area of $\mathcal{S}_{1}$. This follows since $\sqrt{1-2^{-2 R_{1}}}(1-\epsilon)>0$, and therefore every $\mathbf{u} \in \mathscr{D}_{a}\left(\mathbf{s}_{1}\right)$ satisfies $\left|\varangle\left(\mathbf{s}_{1}, \mathbf{u}\right)\right|<\pi / 2$. Hence, the surface area of $\mathscr{D}_{a}^{c}\left(\mathbf{s}_{1}\right)$ is always larger than half the surface area of $\mathcal{S}_{1}$ and therefore

$$
v \leq 2 \cdot \frac{1}{r_{1}^{n-1} C_{n}(\pi)}
$$

Combining (133) with (132) and (131) proves the lemma.

\section{E.5.1 Proof of Lemma E.4}

We begin with the following decomposition

$$
\begin{aligned}
\operatorname{Pr}\left[\mathcal{E}_{\mathbf{X}}\right] & =\operatorname{Pr}\left[\mathcal{E}_{\mathbf{X}} \cap \mathcal{E}_{\mathbf{S}}\right]+\operatorname{Pr}\left[\mathcal{E}_{\mathbf{X}} \cap \mathcal{E}_{\mathbf{S}}^{c}\right] \\
& \leq \operatorname{Pr}\left[\mathcal{E}_{\mathbf{S}}\right]+\operatorname{Pr}\left[\mathcal{E}_{\mathbf{X}_{1}} \cap \mathcal{E}_{\mathbf{S}}^{c}\right]+\operatorname{Pr}\left[\mathcal{E}_{\mathbf{X}_{2}} \cap \mathcal{E}_{\mathbf{S}}^{c}\right]+\operatorname{Pr}\left[\mathcal{E}_{\left(\mathbf{X}_{1}, \mathbf{X}_{2}\right)} \cap \mathcal{E}_{\mathbf{X}_{2}}^{c} \cap \mathcal{E}_{\mathbf{X}_{1}}^{c} \cap \mathcal{E}_{\mathbf{S}}^{c}\right] \\
& \leq \operatorname{Pr}\left[\mathcal{E}_{\mathbf{S}}\right]+\operatorname{Pr}\left[\mathcal{E}_{\mathbf{X}_{1}}\right]+\operatorname{Pr}\left[\mathcal{E}_{\mathbf{X}_{2}}\right]+\operatorname{Pr}\left[\mathcal{E}_{\left(\mathbf{X}_{1}, \mathbf{X}_{2}\right)} \cap \mathcal{E}_{\mathbf{X}_{2}}^{c} \cap \mathcal{E}_{\mathbf{X}_{1}}^{c} \cap \mathcal{E}_{\mathbf{S}}^{c}\right] .
\end{aligned}
$$

The proof of Lemma E.4 now follows by showing that for every $\delta>0$ and $0.3>\epsilon>0$ there exists an $n_{2}^{\prime}(\delta, \epsilon)>0$ such that for all $n>n_{2}^{\prime}(\delta, \epsilon)$

$$
\begin{aligned}
\operatorname{Pr}\left[\mathcal{E}_{\mathbf{X}_{i}}\right] & \leq \delta, \quad i \in\{1,2\} \\
\operatorname{Pr}\left[\mathcal{E}_{\left(\mathbf{X}_{1}, \mathbf{X}_{2}\right)} \cap \mathcal{E}_{\mathbf{S}}^{c} \cap \mathcal{E}_{\mathbf{X}_{1}}^{c} \cap \mathcal{E}_{\mathbf{X}_{2}}^{c}\right] & \leq 3 \delta .
\end{aligned}
$$


Proof of (134): We give the proof for $\mathcal{E}_{\mathbf{X}_{1}}$. Due to the symmetry the proof for $\mathcal{E}_{\mathbf{X}_{2}}$ then follows by similar arguments. Let $\mathcal{E}_{\mathbf{X}_{1}}(j)$ be the event that $\mathbf{U}_{1}(j)$ does not have a typical angle to $\mathbf{S}_{1}$, i.e.

$$
\mathcal{E}_{\mathbf{X}_{1}}(j)=\left\{\left(\mathbf{s}_{1}, \mathbf{s}_{2}, \mathcal{C}_{1}, \mathcal{C}_{2}\right):\left|\cos \varangle\left(\mathbf{u}_{1}(j), \mathbf{s}_{1}\right)-\sqrt{1-2^{-2 R_{1}}}\right|>\epsilon \sqrt{1-2^{-2 R_{1}}}\right\} .
$$

Then,

$$
\begin{aligned}
\operatorname{Pr}\left[\mathcal{E}_{\mathbf{X}_{1}}\right] & =\operatorname{Pr}\left[\mathcal{E}_{\mathbf{X}_{1}} \mid \mathbf{S}_{1}=\mathbf{s}_{1}\right] \\
& =\operatorname{Pr}\left[\bigcap_{j=1}^{2^{n R_{1}}} \mathcal{E}_{\mathbf{X}_{1}}(j) \mid \mathbf{S}_{1}=\mathbf{s}_{1}\right] \\
& =\prod_{j=1}^{2^{n R_{1}}} \operatorname{Pr}\left[\mathcal{E}_{\mathbf{X}_{1}}(j) \mid \mathbf{S}_{1}=\mathbf{s}_{1}\right] \\
& \stackrel{a)}{=} \prod_{j=1}^{2^{n R_{1}}} \operatorname{Pr}\left[\mathcal{E}_{\mathbf{X}_{1}}(j)\right] \\
& \stackrel{b)}{=}\left(\operatorname{Pr}\left[\mathcal{E}_{\mathbf{X}_{1}}(1)\right]\right)^{2^{n R_{1}}} \\
& =\left(1-\operatorname{Pr}\left[\mathcal{E}_{\mathbf{X}_{1}}^{c}(1)\right]\right)^{2^{n R_{1}}}
\end{aligned}
$$

where in $a$ ) we have used that the probability of $\mathcal{E}_{\mathbf{X}_{1}}(j)$ does not depend on $\mathbf{S}_{1}=\mathbf{s}_{1}$, and in $b$ ) we have used that all $\mathbf{U}_{1}(j)$ have the same distribution. To upper bound (136) we now rewrite $\mathcal{E}_{\mathbf{X}_{1}}^{c}(1)$ as

$$
\begin{aligned}
\mathcal{E}_{\mathbf{X}_{1}}^{c}(1) & =\left\{\left(\mathbf{s}_{1}, \mathbf{s}_{2}, \mathcal{C}_{1}, \mathcal{C}_{2}\right):\left|\cos \varangle\left(\mathbf{u}_{1}(1), \mathbf{s}_{1}\right)-\sqrt{1-2^{-2 R_{1}}}\right| \leq \epsilon \sqrt{1-2^{-2 R_{1}}}\right\} \\
& =\left\{\left(\mathbf{s}_{1}, \mathbf{s}_{2}, \mathcal{C}_{1}, \mathcal{C}_{2}\right): \sqrt{1-2^{-2 R_{1}}}(1-\epsilon) \leq \cos \varangle\left(\mathbf{u}_{1}(1), \mathbf{s}_{1}\right) \leq \sqrt{1-2^{-2 R_{1}}}(1+\epsilon)\right\} \\
& =\left\{\left(\mathbf{s}_{1}, \mathbf{s}_{2}, \mathcal{C}_{1}, \mathcal{C}_{2}\right): \cos \theta_{1, \max } \leq \cos \varangle\left(\mathbf{u}_{1}(1), \mathbf{s}_{1}\right) \leq \cos \theta_{1, \min }\right\},
\end{aligned}
$$

where we have used the notation

$$
\cos \theta_{1, \text { max }} \triangleq \sqrt{1-2^{-2 R_{1}}}(1-\epsilon) \quad \text { and } \quad \cos \theta_{1, \text { min }} \triangleq \sqrt{1-2^{-2 R_{1}}}(1+\epsilon) .
$$

Hence, since $\mathbf{U}_{1}(1)$ is generated independently of $\mathbf{S}_{1}$ and distributed uniformly on $\mathcal{S}_{1}$,

$$
\operatorname{Pr}\left[\mathcal{E}_{\mathbf{X}_{1}}^{c}(1)\right]=\frac{C_{n}\left(\theta_{1, \max }\right)-C_{n}\left(\theta_{1, \min }\right)}{C_{n}(\pi)} .
$$

Combining (137) with (136) gives

$$
\begin{aligned}
\operatorname{Pr}\left[\mathcal{E}_{\mathbf{X}_{1}}\right] & =\left(1-\frac{C_{n}\left(\theta_{1, \max }\right)-C_{n}\left(\theta_{1, \min }\right)}{C_{n}(\pi)}\right)^{2^{n R_{1}}} \\
& \stackrel{a)}{\leq}\left(\exp \left(-\frac{C_{n}\left(\theta_{1, \max }\right)-C_{n}\left(\theta_{1, \min }\right)}{C_{n}(\pi)}\right)\right)^{2^{n R_{1}}}
\end{aligned}
$$




$$
\begin{aligned}
& \stackrel{b)}{\leq} \exp \left(-2^{n R_{1}} \frac{\Gamma\left(\frac{n}{2}+1\right)}{n \Gamma\left(\frac{n+1}{2}\right) \sqrt{\pi}}\left(\frac{\sin ^{(n-1)} \theta_{1, \max }}{\cos \theta_{1, \max }}\left(1-\frac{1}{n} \tan ^{2} \theta_{1, \max }\right)-\frac{\sin ^{(n-1)} \theta_{1, \text { min }}}{\cos \theta_{1, \text { min }}}\right)\right) \\
& =\exp \left(-\frac{\Gamma\left(\frac{n}{2}+1\right)}{n \Gamma\left(\frac{n+1}{2}\right) \sqrt{\pi}}\left(\frac{2^{n\left(R_{1}+\log _{2}\left(\sin \theta_{1, \max }\right)\right)}}{\sin \theta_{1, \max } \cos \theta_{1, \max }}\left(1-\frac{1}{n} \tan ^{2} \theta_{1, \max }\right)-\right.\right. \\
& \left.\left.-\frac{2^{n\left(R_{1}+\log _{2}\left(\sin \theta_{1, \min }\right)\right)}}{\sin \theta_{1, \min } \cos \theta_{1, \text { min }}}\right)\right)
\end{aligned}
$$

where in $a$ ) we have used that $1-x \leq \exp (-x)$, and in $b$ ) we have lower bounded $C_{n}\left(\theta_{1, \max }\right) / C_{n}(\pi)$ and upper bounded $C_{n}\left(\theta_{1, \min }\right) / C_{n}(\pi)$ according to Lemma E.17. It now follows from sphere-packing and -covering, see e.g. [25], that for every $\epsilon>0$ we have $\operatorname{Pr}\left[\mathcal{E}_{\mathbf{X}_{1}}\right] \rightarrow 0$ as $n \rightarrow \infty$. More precisely, this holds since the exponent on the RHS of (138) grows exponentially in $n$. This follows since on the one hand for large $n$

$$
\frac{\Gamma\left(\frac{n}{2}+1\right)}{n \Gamma\left(\frac{n+1}{2}\right) \sqrt{\pi}} \approx \frac{1}{\sqrt{n 2 \pi}},
$$

and on the other hand the term

$$
\frac{2^{n\left(R_{1}+\log _{2}\left(\sin \theta_{1, \max }\right)\right)}}{\sin \theta_{1, \max } \cos \theta_{1, \max }}\left(1-\frac{1}{n} \tan ^{2} \theta_{1, \text { max }}\right)-\frac{2^{n\left(R_{1}+\log _{2}\left(\sin \theta_{1, \min }\right)\right)}}{\sin \theta_{1, \min } \cos \theta_{1, \min }}
$$

grows exponentially in $n$. The latter holds since first of all

$$
\left(1-\frac{1}{n} \tan ^{2} \theta_{1, \max }\right) \approx 1 \quad \text { for large } n,
$$

second, the denominators of the fractions are independent of $n$, and third since

$$
R_{1}+\log _{2}\left(\sin \theta_{1, \max }\right) \geq R_{1}+\log _{2}\left(\sin \theta_{1, \min }\right),
$$

with $R_{1}+\log _{2}\left(\sin \theta_{1, \max }\right)>0$. That $R_{1}+\log _{2}\left(\sin \theta_{1, \max }\right)>0$ can be seen as follows.

$$
\begin{aligned}
-\log _{2}\left(\sin \theta_{1, \max }\right) & =-\log _{2}\left(\sqrt{1-\cos ^{2} \theta_{1, \max }}\right) \\
& \stackrel{a)}{=}-\frac{1}{2} \log _{2}\left(2^{-2 R_{1}}+\epsilon(2-\epsilon)\left(1-2^{-2 R_{1}}\right)\right) \\
& <-\frac{1}{2} \log _{2}\left(2^{-2 R_{1}}\right) \\
& =R_{1},
\end{aligned}
$$

where in $a$ ) we have used the definition of $\cos \theta_{1, \max }$.

Proof of (135): By the notation in (128) we have

$$
\begin{aligned}
\cos \varangle\left(\mathbf{u}_{1}^{*}, \mathbf{u}_{2}^{*}\right) & =\frac{\left\langle\mathbf{u}_{1}^{*}, \mathbf{u}_{2}^{*}\right\rangle}{\left\|\mathbf{u}_{1}^{*}\right\|\left\|\mathbf{u}_{2}^{*}\right\|} \\
& =\frac{1}{\left\|\mathbf{u}_{1}^{*}\right\|\left\|\mathbf{u}_{2}^{*}\right\|}\left(\nu_{1} \nu_{2}\left\langle\mathbf{s}_{1}, \mathbf{s}_{2}\right\rangle+\nu_{1}\left\langle\mathbf{s}_{1}, \mathbf{v}_{2}\right\rangle+\nu_{2}\left\langle\mathbf{v}_{1}, \mathbf{s}_{2}\right\rangle+\left\langle\mathbf{v}_{1}, \mathbf{v}_{2}\right\rangle\right),
\end{aligned}
$$


where we recall that $\nu_{1}$ is a function of $\left\|\mathbf{s}_{1}\right\|$ and $\cos \varangle\left(\mathbf{s}_{1}, \mathbf{u}_{1}^{*}\right)$ and similarly $\nu_{2}$ is a function of $\left\|\mathbf{s}_{2}\right\|$ and $\cos \varangle\left(\mathbf{s}_{2}, \mathbf{u}_{2}^{*}\right)$. Now, define the four events

$$
\begin{aligned}
& \mathcal{A}_{1}=\left\{\left(\mathbf{s}_{1}, \mathbf{s}_{2}, \mathcal{C}_{1}, \mathcal{C}_{2}\right):\left|\tilde{\rho}-\frac{\nu_{1} \nu_{2}}{\left\|\mathbf{u}_{1}^{*}\right\|\left\|\mathbf{u}_{2}^{*}\right\|}\left\langle\mathbf{s}_{1}, \mathbf{s}_{2}\right\rangle\right|>4 \epsilon\right\} \\
& \mathcal{A}_{2}=\left\{\left(\mathbf{s}_{1}, \mathbf{s}_{2}, \mathcal{C}_{1}, \mathcal{C}_{2}\right):\left|\frac{\nu_{1}}{\left\|\mathbf{u}_{1}^{*}\right\|\left\|\mathbf{u}_{2}^{*}\right\|}\left\langle\mathbf{s}_{1}, \mathbf{v}_{2}\right\rangle\right|>\epsilon\right\} \\
& \mathcal{A}_{3}=\left\{\left(\mathbf{s}_{1}, \mathbf{s}_{2}, \mathcal{C}_{1}, \mathcal{C}_{2}\right):\left|\frac{\nu_{2}}{\left\|\mathbf{u}_{1}^{*}\right\|\left\|\mathbf{u}_{2}^{*}\right\|}\left\langle\mathbf{s}_{2}, \mathbf{v}_{1}\right\rangle\right|>\epsilon\right\} \\
& \mathcal{A}_{4}=\left\{\left(\mathbf{s}_{1}, \mathbf{s}_{2}, \mathcal{C}_{1}, \mathcal{C}_{2}\right):\left|\frac{1}{\left\|\mathbf{u}_{1}^{*}\right\|\left\|\mathbf{u}_{2}^{*}\right\|}\left\langle\mathbf{v}_{1}, \mathbf{v}_{2}\right\rangle\right|>\epsilon\right\} .
\end{aligned}
$$

Note that by (139), $\mathcal{E}_{\left(\mathbf{X}_{1}, \mathbf{X}_{2}\right)} \subset\left(\mathcal{A}_{1} \cup \mathcal{A}_{2} \cup \mathcal{A}_{3} \cup \mathcal{A}_{4}\right)$. Thus,

$$
\begin{aligned}
\operatorname{Pr}\left[\mathcal{E}_{\left(\mathbf{X}_{1}, \mathbf{X}_{2}\right)} \cap \mathcal{E}_{\mathbf{S}}^{c} \cap \mathcal{E}_{\mathbf{X}_{1}}^{c} \cap \mathcal{E}_{\mathbf{X}_{2}}^{c}\right] \leq & \operatorname{Pr}\left[\mathcal{A}_{1} \cap \mathcal{E}_{\mathbf{S}}^{c} \cap \mathcal{E}_{\mathbf{X}_{1}}^{c} \cap \mathcal{E}_{\mathbf{X}_{2}}^{c}\right]+\operatorname{Pr}\left[\mathcal{A}_{2} \cap \mathcal{E}_{\mathbf{S}}^{c} \cap \mathcal{E}_{\mathbf{X}_{1}}^{c} \cap \mathcal{E}_{\mathbf{X}_{2}}^{c}\right] \\
& +\operatorname{Pr}\left[\mathcal{A}_{3} \cap \mathcal{E}_{\mathbf{S}}^{c} \cap \mathcal{E}_{\mathbf{X}_{1}}^{c} \cap \mathcal{E}_{\mathbf{X}_{2}}^{c}\right]+\operatorname{Pr}\left[\mathcal{A}_{4} \cap \mathcal{E}_{\mathbf{S}}^{c} \cap \mathcal{E}_{\mathbf{X}_{1}}^{c} \cap \mathcal{E}_{\mathbf{X}_{2}}^{c}\right] \\
\leq & \operatorname{Pr}\left[\mathcal{A}_{1} \mid \mathcal{E}_{\mathbf{S}}^{c} \cap \mathcal{E}_{\mathbf{X}_{1}}^{c} \cap \mathcal{E}_{\mathbf{X}_{2}}^{c}\right]+\operatorname{Pr}\left[\mathcal{A}_{2} \mid \mathcal{E}_{\mathbf{S}}^{c}\right]+\operatorname{Pr}\left[\mathcal{A}_{3} \mid \mathcal{E}_{\mathbf{S}}^{c}\right] \\
& +\operatorname{Pr}\left[\mathcal{A}_{4} \mid \mathcal{E}_{\mathbf{S}}^{c}\right] .
\end{aligned}
$$

The four terms on the RHS of (140) are now bounded in the following two lemmas.

Lemma E.20. For $\epsilon \leq 0.3$

$$
\operatorname{Pr}\left[\mathcal{A}_{1} \mid \mathcal{E}_{\mathbf{S}}^{c} \cap \mathcal{E}_{\mathbf{X}_{1}}^{c} \cap \mathcal{E}_{\mathbf{X}_{2}}^{c}\right]=0 .
$$

Proof. We first note that the term in the definition of $\mathcal{A}_{1}$ can be rewritten as

$$
\frac{\nu_{1} \nu_{2}}{\left\|\mathbf{u}_{1}^{*}\right\|\left\|\mathbf{u}_{2}^{*}\right\|}\left\langle\mathbf{s}_{1}, \mathbf{s}_{2}\right\rangle=\cos \varangle\left(\mathbf{s}_{1}, \mathbf{u}_{1}^{*}\right) \cos \varangle\left(\mathbf{s}_{2}, \mathbf{u}_{2}^{*}\right) \cos \varangle\left(\mathbf{s}_{1}, \mathbf{s}_{2}\right) .
$$

We can now upper and lower bound the RHS of (141) for $\left(\mathbf{s}_{1}, \mathbf{s}_{2}, \mathcal{C}_{1}, \mathcal{C}_{2}\right) \in \mathcal{E}_{\mathbf{S}}^{c} \cap \mathcal{E}_{\mathbf{X}_{1}}^{c} \cap \mathcal{E}_{\mathbf{X}_{2}}^{c}$ by noticing that $\left(\mathbf{s}_{1}, \mathbf{s}_{2}, \mathcal{C}_{1}, \mathcal{C}_{2}\right) \in \mathcal{E}_{\mathbf{S}}^{c}$ implies

$$
\left|\cos \varangle\left(\mathbf{s}_{1}, \mathbf{s}_{2}\right)-\rho\right|<\rho \epsilon,
$$

that $\left(\mathbf{s}_{1}, \mathbf{s}_{2}, \mathcal{C}_{1}, \mathcal{C}_{2}\right) \in \mathcal{E}_{\mathbf{X}_{1}}^{c}$ implies

$$
\left|\sqrt{1-2^{-2 R_{1}}}-\cos \varangle\left(\mathbf{s}_{1}, \mathbf{u}_{1}^{*}\right)\right|<\epsilon \sqrt{1-2^{-2 R_{1}}},
$$

and that $\left(\mathbf{s}_{1}, \mathbf{s}_{2}, \mathcal{C}_{1}, \mathcal{C}_{2}\right) \in \mathcal{E}_{\mathbf{X}_{2}}^{c}$ implies

$$
\left|\sqrt{1-2^{-2 R_{2}}}-\cos \varangle\left(\mathbf{s}_{2}, \mathbf{u}_{2}^{*}\right)\right|<\epsilon \sqrt{1-2^{-2 R_{2}}} .
$$

Hence, combined with (141) this gives

$$
\tilde{\rho}(1-\epsilon)^{3} \leq \frac{\nu_{1} \nu_{2}}{\left\|\mathbf{u}_{1}^{*}\right\|\left\|\mathbf{u}_{2}^{*}\right\|}\left\langle\mathbf{s}_{1}, \mathbf{s}_{2}\right\rangle \leq \tilde{\rho}(1+\epsilon)^{3}, \quad\left(\mathbf{s}_{1}, \mathbf{s}_{2}, \mathcal{C}_{1}, \mathcal{C}_{2}\right) \in \mathcal{E}_{\mathbf{S}}^{c} \cap \mathcal{E}_{\mathbf{X}_{1}}^{c} \cap \mathcal{E}_{\mathbf{X}_{2}}^{c} .
$$

The LHS can be lower bounded by $\tilde{\rho}(1-3 \epsilon) \leq \tilde{\rho}(1-\epsilon)^{3}$, and the RHS can be upper bounded by $\tilde{\rho}(1+\epsilon)^{3} \leq \tilde{\rho}(1+4 \epsilon)$ whenever $\epsilon \leq 0.3$. Hence, for $\epsilon \leq 0.3$

$$
\left|\tilde{\rho}-\frac{\nu_{1} \nu_{2}}{\left\|\mathbf{u}_{1}^{*}\right\|\left\|\mathbf{u}_{2}^{*}\right\|}\left\langle\mathbf{s}_{1}, \mathbf{s}_{2}\right\rangle\right| \leq 4 \tilde{\rho} \epsilon \leq 4 \epsilon \text {. }
$$


Lemma E.21. For every $\delta>0$ and $\epsilon>0$ there exists an $n_{\mathcal{A}}^{\prime}(\delta, \epsilon)$ such that for all $n>n_{\mathcal{A}}^{\prime}(\delta, \epsilon)$

$$
\operatorname{Pr}\left[\mathcal{A}_{2} \mid \mathcal{E}_{\mathbf{S}}^{c}\right]<\delta, \quad \operatorname{Pr}\left[\mathcal{A}_{3} \mid \mathcal{E}_{\mathbf{S}}^{c}\right]<\delta, \quad \operatorname{Pr}\left[\mathcal{A}_{4} \mid \mathcal{E}_{\mathbf{S}}^{c}\right]<\delta .
$$

Proof. We start derivation of the bound on $\mathcal{A}_{2}$. To this end, we first upper bound the inner product between $\mathbf{s}_{1}$ and $\mathbf{v}_{2}$. Let $\mathbf{s}_{1, \mathrm{P}}$ denote the projection of $\mathbf{s}_{1}$ onto the subspace of $\mathbb{R}^{n}$ that is orthogonal to $\mathbf{s}_{2}$, and that thus contains $\mathbf{v}_{2}$. Hence,

$$
\begin{aligned}
\left|\frac{\nu_{1}}{\left\|\mathbf{u}_{1}^{*}\right\|\left\|\mathbf{u}_{2}^{*}\right\|}\left\langle\mathbf{s}_{1}, \mathbf{v}_{2}\right\rangle\right| & \stackrel{a)}{=}\left|\cos \varangle\left(\mathbf{s}_{1}, \mathbf{u}_{1}^{*}\right)\left\langle\frac{\mathbf{s}_{1}}{\left\|\mathbf{s}_{1}\right\|}, \frac{\mathbf{v}_{2}}{\left\|\mathbf{u}_{2}^{*}\right\|}\right\rangle\right| \\
& \stackrel{b)}{\leq}\left|\cos \varangle\left(\mathbf{s}_{1}, \mathbf{u}_{1}^{*}\right)\right|\left\langle\frac{\mathbf{s}_{1}}{\left\|\mathbf{s}_{1}\right\|}, \frac{\mathbf{v}_{2}}{\left\|\mathbf{v}_{2}\right\|}\right\rangle \mid \\
& \leq\left|\left\langle\frac{\mathbf{s}_{1}}{\left\|\mathbf{s}_{1}\right\|}, \frac{\mathbf{v}_{2}}{\left\|\mathbf{v}_{2}\right\|}\right\rangle\right| \\
& =\left|\left\langle\frac{\mathbf{s}_{1, \mathrm{P}}}{\left\|\mathbf{s}_{1}\right\|}, \frac{\mathbf{v}_{2}}{\left\|\mathbf{v}_{2}\right\|}\right\rangle\right| \\
& \leq\left|\left\langle\frac{\mathbf{s}_{1, P}}{\left\|\mathbf{s}_{1, \mathrm{P}}\right\|}, \frac{\mathbf{v}_{2}}{\left\|\mathbf{v}_{2}\right\|}\right\rangle\right| \\
& =\left|\cos \varangle\left(\mathbf{s}_{1, \mathrm{P}}, \mathbf{v}_{2}\right)\right|,
\end{aligned}
$$

where a) follows by the definition of $\nu_{1}$ and b) follows since by the definition of $\mathbf{v}_{2}$ we have $\left\|\mathbf{v}_{2}\right\| \leq\left\|\mathbf{u}_{2}^{*}\right\|$. By (142) it now follows that

$$
\begin{aligned}
\operatorname{Pr}\left[\mathcal{A}_{2} \mid \mathcal{E}_{\mathbf{S}}^{c}\right] & \leq \operatorname{Pr}\left[\left(\mathbf{S}_{1}, \mathbf{S}_{2}, \mathscr{C}_{1}, \mathscr{C}_{2}\right):\left|\cos \varangle\left(\mathbf{S}_{1, \mathrm{P}}, \mathbf{V}_{2}\right)\right|>\epsilon \mid \mathcal{E}_{\mathbf{S}}^{c}\right] \\
& \leq \operatorname{Pr}\left[\left(\mathbf{S}_{1}, \mathbf{S}_{2}, \mathscr{C}_{1}, \mathscr{C}_{2}\right):\left|\frac{\pi}{2}-\varangle\left(\mathbf{S}_{1, \mathrm{P}}, \mathbf{V}_{2}\right)\right|>\epsilon \mid \mathcal{E}_{\mathbf{S}}^{c}\right] \\
& =\mathrm{E}_{\mathbf{S}_{1}, \mathbf{S}_{2}}\left[\operatorname{Pr}_{\mathscr{C}_{1}, \mathscr{C}_{2}}\left(\left|\frac{\pi}{2}-\varangle\left(\mathbf{s}_{1, \mathrm{P}}, \mathbf{V}_{2}\right)\right|>\epsilon \mid\left(\mathbf{S}_{1}, \mathbf{S}_{2}\right)=\left(\mathbf{s}_{1}, \mathbf{S}_{2}\right), \mathcal{E}_{\mathbf{S}}^{c}\right)\right],
\end{aligned}
$$

where in the last line we have denoted by $\operatorname{Pr}_{\mathscr{C}_{1}, \mathscr{C}_{2}}(\cdots \mid \cdots)$ the conditional probability of the codebooks $\mathscr{C}_{1}$ and $\mathscr{C}_{2}$ being such that $\left|\pi / 2-\varangle\left(\mathbf{s}_{1, \mathrm{P}}, \mathbf{V}_{2}\right)\right|>\epsilon$ given $\left(\mathbf{S}_{1}, \mathbf{S}_{2}\right)=$ $\left(\mathbf{s}_{1}, \mathbf{s}_{2}\right)$ and $\left(\mathbf{s}_{1}, \mathbf{s}_{2}\right) \in \mathcal{E}_{\mathbf{S}}^{c}$. To conclude our bound we now notice that conditioned on $\left(\mathbf{S}_{1}, \mathbf{S}_{2}\right)=\left(\mathbf{s}_{1}, \mathbf{s}_{2}\right)$, the random vector $\mathbf{V}_{2} /\left\|\mathbf{V}_{2}\right\|$ is distributed uniformly on the surface of the centered $\mathbb{R}^{n-1}$-sphere of unit radius that lies in the subspace that is orthogonal to $\mathbf{s}_{2}$. Hence,

$$
\begin{aligned}
\operatorname{Pr}\left[\mathcal{A}_{2} \mid \mathcal{E}_{\mathbf{S}}^{c}\right] & \leq \mathrm{E}_{\mathbf{S}_{1}, \mathbf{S}_{2}}\left[\frac{2 C_{n-1}(\pi / 2-\epsilon)}{C_{n-1}(\pi)} \mid \mathcal{E}_{\mathbf{S}}^{c}\right] \\
& \leq \frac{2 C_{n-1}(\pi / 2-\epsilon)}{C_{n-1}(\pi)} \\
& \leq \frac{2 \Gamma\left(\frac{n+1}{2}\right)}{(n-1) \Gamma\left(\frac{n}{2}\right) \sqrt{\pi}} \frac{\sin ^{(n-2)}(\pi / 2-\epsilon)}{\cos (\pi / 2-\epsilon)} \\
& \leq \frac{2 \Gamma\left(\frac{n+1}{2}\right)}{(n-1) \Gamma\left(\frac{n}{2}\right) \sqrt{\pi} \cos (\pi / 2-\epsilon)} .
\end{aligned}
$$


Upper bounding the ratio of Gamma functions by the asymptotic series of Lemma E.18, gives for every $\epsilon>0$ that $\operatorname{Pr}\left[\mathcal{A}_{2} \mid \mathcal{E}_{\mathbf{S}}^{c}\right] \rightarrow 0$ as $n \rightarrow \infty$. By similar arguments it also follows that $\operatorname{Pr}\left[\mathcal{A}_{3} \mid \mathcal{E}_{\mathrm{S}}^{c}\right] \rightarrow 0$ as $n \rightarrow \infty$.

To conclude the proof of Lemma E.21, we derive the bound on $\mathcal{A}_{4}$. The derivations are similar to those for $\mathcal{A}_{2}$. First, define by $\mathbf{v}_{1, \mathrm{P}}$ the projection of $\mathbf{v}_{1}$ onto the subspace of $\mathbb{R}^{n}$ that is orthogonal to $\mathbf{s}_{2}$. As in (142) we can show that

$$
\left|\frac{1}{\left\|\mathbf{u}_{1}^{*}\right\|\left\|\mathbf{u}_{2}^{*}\right\|}\left\langle\mathbf{v}_{1}, \mathbf{v}_{2}\right\rangle\right| \leq\left|\cos \varangle\left(\mathbf{v}_{1, \mathrm{P}}, \mathbf{v}_{2}\right)\right|,
$$

from which it then follows, using $|\cos x| \leq|\pi / 2-x|$, that

$$
\operatorname{Pr}\left[\mathcal{A}_{4} \mid \mathcal{E}_{\mathbf{S}}^{c}\right] \leq \mathrm{E}_{\mathbf{S}_{1}, \mathbf{S}_{2}, \mathscr{C}_{1}}\left[\operatorname{Pr}_{\mathscr{C}_{2}}\left(\left|\frac{\pi}{2}-\varangle\left(\mathbf{v}_{1, \mathrm{P}}, \mathbf{V}_{2}\right)\right|>\epsilon \mid\left(\mathbf{S}_{1}, \mathbf{S}_{2}\right)=\left(\mathbf{s}_{1}, \mathbf{s}_{2}\right), \mathscr{C}_{1}=\mathcal{C}_{1}, \mathcal{E}_{\mathbf{S}}^{c}\right)\right]
$$

The desired bound now follows from noticing that conditioned on $\left(\mathbf{S}_{1}, \mathbf{S}_{2}\right)=\left(\mathbf{s}_{1}, \mathbf{s}_{2}\right)$ and $\mathscr{C}_{1}=\mathcal{C}_{1}$, the random vector $\mathbf{V}_{2} /\left\|\mathbf{V}_{2}\right\|$ is distributed uniformly on the surface of the centered $\mathbb{R}^{n-1}$-sphere of unit radius that lies in the subspace that is orthogonal to $\mathbf{s}_{2}$. Hence, similarly as in the derivation for $\mathcal{A}_{2}$

$$
\begin{aligned}
\operatorname{Pr}\left[\mathcal{A}_{4} \mid \mathcal{E}_{\mathbf{S}}^{c}\right] & \leq \mathrm{E}_{\mathbf{S}_{1}, \mathbf{S}_{2}, \mathscr{C}_{1}}\left[\frac{2 C_{n-1}(\pi / 2-\epsilon)}{C_{n-1}(\pi)} \mid \mathcal{E}_{\mathbf{S}}^{c}\right] \\
& \leq \frac{2 \Gamma\left(\frac{n+1}{2}\right)}{(n-1) \Gamma\left(\frac{n}{2}\right) \sqrt{\pi} \cos (\pi / 2-\epsilon)} .
\end{aligned}
$$

Upper bounding the ratio of Gamma functions by the asymptotic series of Lemma E.18, gives for every $\epsilon>0$ that $\operatorname{Pr}\left[\mathcal{A}_{4} \mid \mathcal{E}_{\mathbf{S}}^{c}\right] \rightarrow 0$ as $n \rightarrow \infty$.

Combining Lemma E.20 and Lemma E.21 with (140) gives that for every $\delta>0$ and $0.3>\epsilon>0$ there exists an $n_{\mathcal{A}}^{\prime}(\delta, \epsilon)$ such that for all $n>n_{\mathcal{A}}^{\prime}(\delta, \epsilon)$

$$
\operatorname{Pr}\left[\mathcal{E}_{\left(\mathbf{X}_{1}, \mathbf{X}_{2}\right)} \cap \mathcal{E}_{\mathbf{S}}^{c} \cap \mathcal{E}_{\mathbf{X}_{1}}^{c} \cap \mathcal{E}_{\mathbf{X}_{2}}^{c}\right] \leq 3 \delta
$$

\section{E.5.2 Proof of Lemma E.7}

The proof follows from upper bounding $\operatorname{Pr}\left[\mathcal{G} \mid \mathcal{E}_{\mathbf{X}_{1}}^{c}\right]$ as a function of $R_{1}$. First, note that

$$
\begin{aligned}
\operatorname{Pr}\left[\mathcal{G} \mid \mathcal{E}_{\mathbf{X}_{1}}^{c}\right] & =\operatorname{Pr}\left[\mathcal{G} \mid \mathbf{U}_{1}^{*} \neq \mathbf{0}\right] \\
& =\operatorname{Pr}\left[\mathcal{G} \mid \varsigma_{1}\left(\mathbf{S}_{1}, \mathscr{C}_{1}\right)=1\right],
\end{aligned}
$$

where the second equality holds because the conditional distribution of the codewords conditional on $\mathbf{u}_{1}^{*} \neq \mathbf{0}$ is invariant with respect to permutations of the indexing of the codewords. The desired upper bound is now obtained by decomposing $\mathcal{G}$ into sub-events $\mathcal{G}_{j}, j \in\left\{2,3, \ldots, 2^{n R_{1}}\right\}$, where

$$
\mathcal{G}_{j} \triangleq\left\{\left(\mathbf{s}_{1}, \mathbf{s}_{2}, \mathcal{C}_{1}, \mathcal{C}_{2}, \mathbf{z}\right): \cos \varangle\left(\mathbf{w}, \mathbf{u}_{1}(j)\right) \geq \Delta\right\} .
$$


By (144) we now have

$$
\begin{aligned}
\operatorname{Pr}\left[\mathcal{G} \mid \mathcal{E}_{\mathbf{X}_{1}}^{c}\right] & =\operatorname{Pr}\left[\bigcup_{j=2}^{2^{n R_{1}}} \mathcal{G}_{j} \mid \varsigma_{1}\left(\mathbf{S}_{1}, \mathscr{C}_{1}\right)=1\right] \\
& \leq \sum_{j=2}^{2^{n R_{1}}} \operatorname{Pr}\left[\mathcal{G}_{j} \mid \varsigma_{1}\left(\mathbf{S}_{1}, \mathscr{C}_{1}\right)=1\right] \\
& <2^{n R_{1}} \operatorname{Pr}\left[\mathcal{G}_{2} \mid \varsigma_{1}\left(\mathbf{S}_{1}, \mathscr{C}_{1}\right)=1\right] \\
& \leq 2^{n R_{1}} \cdot 2 \frac{C_{n}(\arccos \Delta)}{C_{n}(\pi)}
\end{aligned}
$$

where in the third step we have used that $\operatorname{Pr}\left[\mathcal{G}_{j} \mid \varsigma_{i}\left(\mathbf{s}_{i}, \mathcal{C}_{i}\right)=1\right]$ is the same for all $j \in$ $\left\{2,3, \ldots 2^{n R_{1}}\right\}$ because the conditional distribution of $\mathbf{u}_{1}(j)$ given $\varsigma_{i}\left(\mathbf{s}_{i}, \mathcal{C}_{i}\right)=1$ does not depend on $j \in\left\{2,3, \ldots, 2^{n R_{1}}\right\}$ and where in the last step we have upper bounded the density of $\mathbf{U}_{1}(2)$, conditional on $\varsigma_{1}\left(\mathbf{S}_{1}, \mathscr{C}_{1}\right)=1$, by Lemma E.19. Thus, combining (145) with Lemma E.17 gives

$$
\begin{aligned}
\operatorname{Pr}\left[\mathcal{G} \mid \mathcal{E}_{\mathbf{X}_{1}}^{c}\right] & \leq 2^{n R_{1}} \cdot 2 \frac{\Gamma\left(\frac{n}{2}+1\right)\left(1-\Delta^{2}\right)^{(n-1) / 2}}{n \Gamma\left(\frac{n+1}{2}\right) \sqrt{\pi} \Delta} \\
& =\frac{2 \Gamma\left(\frac{n}{2}+1\right)}{n \Gamma\left(\frac{n+1}{2}\right) \sqrt{\pi} \Delta \sqrt{1-\Delta^{2}}} 2^{n\left(R_{1}+1 / 2 \log _{2}\left(1-\Delta^{2}\right)\right)}
\end{aligned}
$$

Replacing the ratio of the Gamma functions by the asymptotic series of Lemma E.18 establishes (111).

\section{E.5.3 Proof of Lemma E.9}

The proof follows by upper bounding $\operatorname{Pr}\left[\mathcal{G} \mid \mathcal{E}_{\mathbf{X}_{1}}^{c} \cap \mathcal{E}_{\mathbf{X}_{2}}^{c}\right]$ as a function of $R_{1}+R_{2}$. To this end, define

$$
\tilde{\varsigma}\left(\mathbf{s}_{1}, \mathbf{s}_{2}, \mathcal{C}_{1}, \mathcal{C}_{2}\right) \triangleq\left(\varsigma_{1}\left(\mathbf{s}_{1}, \mathcal{C}_{1}\right), \varsigma_{2}\left(\mathbf{s}_{2}, \mathcal{C}_{2}\right)\right)
$$

By a symmetry argument, which is similar to the one in the proof of Lemma E.7, we obtain

$$
\operatorname{Pr}\left[\mathcal{G} \mid \mathcal{E}_{\mathbf{X}_{1}}^{c} \cap \mathcal{E}_{\mathbf{X}_{2}}^{c}\right]=\operatorname{Pr}\left[\mathcal{G} \mid \tilde{\varsigma}\left(\mathbf{S}_{1}, \mathbf{S}_{2}, \mathscr{C}_{1}, \mathscr{C}_{2}\right)=(1,1)\right]
$$

The desired upper bound is now obtained by decomposing $\mathcal{G}$ into subevents $\mathcal{G}_{j, \ell}$, where

$$
\mathcal{G}_{j, \ell}=\left\{\left(\mathbf{s}_{1}, \mathbf{s}_{2}, \mathcal{C}_{1}, \mathcal{C}_{2}, \mathbf{z}\right): \cos \varangle\left(\mathbf{u}_{1}(j), \mathbf{u}_{2}(\ell)\right) \geq \Theta, \cos \varangle\left(\mathbf{y}, \alpha_{1} \mathbf{u}_{1}(j)+\alpha_{2} \mathbf{u}_{2}(\ell)\right) \geq \Delta\right\},
$$


for $j \in\left\{2,3, \ldots 2^{n R_{1}}\right\}$ and $\ell \in\left\{2,3, \ldots 2^{n R_{2}}\right\}$. Hence, by (146)

$$
\begin{aligned}
\operatorname{Pr}\left[\mathcal{G} \mid \mathcal{E}_{\mathbf{X}_{1}}^{c} \cap \mathcal{E}_{\mathbf{X}_{2}}^{c}\right] & =\operatorname{Pr}\left[\bigcup_{j=2}^{2^{n R_{1}}} \bigcup_{\ell=2}^{2^{n R_{2}}} \mathcal{G}_{j, \ell} \mid \tilde{\varsigma}\left(\mathbf{S}_{1}, \mathbf{S}_{2}, \mathscr{C}_{1}, \mathscr{C}_{2}\right)=(1,1)\right] \\
& \leq \sum_{j=2}^{2^{n R_{1}}} \sum_{\ell=2}^{2^{n R_{2}}} \operatorname{Pr}\left[\mathcal{G}_{j, \ell} \mid \tilde{\varsigma}\left(\mathbf{S}_{1}, \mathbf{S}_{2}, \mathscr{C}_{1}, \mathscr{C}_{2}\right)=(1,1)\right] \\
& <2^{n\left(R_{1}+R_{2}\right)} \operatorname{Pr}\left[\mathcal{G}_{2,2} \mid \tilde{\varsigma}\left(\mathbf{S}_{1}, \mathbf{S}_{2}, \mathscr{C}_{1}, \mathscr{C}_{2}\right)=(1,1)\right]
\end{aligned}
$$

where $a$ ) follows since conditioned on $\tilde{\varsigma}\left(\mathbf{S}_{1}, \mathbf{S}_{2}, \mathscr{C}_{1}, \mathscr{C}_{2}\right)=(1,1)$, the laws of $\varangle\left(\mathbf{U}_{1}(j), \mathbf{U}_{2}(\ell)\right)$ and $\varangle\left(\mathbf{Y}, \alpha_{1} \mathbf{U}_{1}(j)+\alpha_{2} \mathbf{U}_{2}(\ell)\right)$ do not depend on $j \in\left\{2,3, \ldots, 2^{n R_{1}}\right\}$ or $\ell \in\left\{2,3, \ldots, 2^{n R_{2}}\right\}$. We now rewrite the probability $\operatorname{Pr}\left[\mathcal{G}_{2,2} \mid \tilde{\varsigma}\left(\mathbf{S}_{1}, \mathbf{S}_{2}, \mathscr{C}_{1}, \mathscr{C}_{2}\right)=(1,1)\right]$ :

$$
\begin{aligned}
& \operatorname{Pr}\left[\mathcal{G}_{2,2} \mid \tilde{\varsigma}\left(\mathbf{S}_{1}, \mathbf{S}_{2}, \mathscr{C}_{1}, \mathscr{C}_{2}\right)=(1,1)\right] \\
& =\operatorname{Pr}\left[\left(\mathbf{S}_{1}, \mathbf{S}_{2}, \mathbf{U}_{1}(1), \mathbf{U}_{2}(1), \mathbf{U}_{1}(2), \mathbf{U}_{2}(2), \mathbf{Z}\right) \text { are such that } \mathcal{G}_{2,2}\right. \text { occurs } \\
& \left.\mid \tilde{\varsigma}\left(\mathbf{S}_{1}, \mathbf{S}_{2}, \mathscr{C}_{1}, \mathscr{C}_{2}\right)=(1,1)\right] \\
& =\int_{\substack{\left.\left(\mathbf{s}_{1}, \mathbf{s}_{2}\right) \in \mathbb{R}^{n} \times \mathbb{R}^{n} \\
\mathbf{z} \in \mathbf{u}_{2}\right) \in \mathcal{R}_{1} \times \mathcal{S}_{2}}} f\left(\left(\mathbf{S}_{1}, \mathbf{S}_{2}, \mathbf{U}_{1}(1), \mathbf{U}_{2}(1), \mathbf{Z}\right)=\left(\mathbf{s}_{1}, \mathbf{s}_{2}, \mathbf{u}_{1}, \mathbf{u}_{2}, \mathbf{z}\right)\right. \\
& \left.\mid \tilde{\varsigma}\left(\mathbf{S}_{1}, \mathbf{S}_{2}, \mathscr{C}_{1}, \mathscr{C}_{2}\right)=(1,1)\right) \\
& \operatorname{Pr}\left[\cos \varangle\left(\mathbf{U}_{1}(2), \mathbf{U}_{2}(2)\right) \geq \Theta, \cos \varangle\left(\mathbf{y}, \alpha_{1} \mathbf{U}_{1}(2)+\alpha_{2} \mathbf{U}_{2}(2)\right) \geq \Delta\right. \\
& \left.\mid \tilde{\varsigma}\left(\mathbf{S}_{1}, \mathbf{S}_{2}, \mathscr{C}_{1}, \mathscr{C}_{2}\right)=(1,1),\left(\mathbf{S}_{1}, \mathbf{S}_{2}, \mathbf{U}_{1}(1), \mathbf{U}_{2}(1), \mathbf{Z}\right)=\left(\mathbf{s}_{1}, \mathbf{s}_{2}, \mathbf{u}_{1}, \mathbf{u}_{2}, \mathbf{z}\right)\right] \\
& \left(\mathbf{s}_{1}, \mathbf{s}_{2}, \mathbf{u}_{1}, \mathbf{u}_{2}, \mathbf{z}\right) \\
& =\int_{\substack{\left(\mathbf{s}_{1}, \mathbf{s}_{2}\right) \in \mathbb{R}^{n} \times \mathbb{R}^{n} \\
\left(\mathbf{u}_{1}, \mathbf{u}_{2}\right) \in \mathcal{S}_{1} \times \mathcal{S}_{2}}} f\left(\left(\mathbf{S}_{1}, \mathbf{S}_{2}, \mathbf{U}_{1}(1), \mathbf{U}_{2}(1)\right)=\left(\mathbf{s}_{1}, \mathbf{s}_{2}, \mathbf{u}_{1}, \mathbf{u}_{2}\right)\right. \\
& \left.\mid \tilde{\varsigma}\left(\mathbf{S}_{1}, \mathbf{S}_{2}, \mathscr{C}_{1}, \mathscr{C}_{2}\right)=(1,1)\right) \\
& \operatorname{Pr}\left[\cos \varangle\left(\mathbf{U}_{1}(2), \mathbf{U}_{2}(2)\right) \geq \Theta, \cos \varangle\left(\mathbf{y}, \alpha_{1} \mathbf{U}_{1}(2)+\alpha_{2} \mathbf{U}_{2}(2)\right) \geq \Delta\right. \\
& \left.\mid \tilde{\varsigma}\left(\mathbf{S}_{1}, \mathbf{S}_{2}, \mathscr{C}_{1}, \mathscr{C}_{2}\right)=(1,1),\left(\mathbf{S}_{1}, \mathbf{S}_{2}, \mathbf{U}_{1}(1), \mathbf{U}_{2}(1)\right)=\left(\mathbf{s}_{1}, \mathbf{s}_{2}, \mathbf{u}_{1}, \mathbf{u}_{2}\right)\right] \\
& \left(\mathbf{s}_{1}, \mathbf{s}_{2}, \mathbf{u}_{1}, \mathbf{u}_{2}\right) \text {, }
\end{aligned}
$$

where in the last step we have used that the probability term does not depend on $\mathbf{z}$. To upper bound the integral we now upper bound this probability term.

$$
\begin{gathered}
\operatorname{Pr}\left[\cos \varangle\left(\mathbf{U}_{1}(2), \mathbf{U}_{2}(2)\right) \geq \Theta, \cos \varangle\left(\mathbf{y}, \alpha_{1} \mathbf{U}_{1}(2)+\alpha_{2} \mathbf{U}_{2}(2)\right) \geq \Delta\right. \\
\mid \begin{array}{r}
\left.\tilde{\varsigma}\left(\mathbf{S}_{1}, \mathbf{S}_{2}, \mathscr{C}_{1}, \mathscr{C}_{2}\right)=(1,1),\left(\mathbf{S}_{1}, \mathbf{S}_{2}, \mathbf{U}_{1}(1), \mathbf{U}_{2}(1)\right)=\left(\mathbf{s}_{1}, \mathbf{s}_{2}, \mathbf{u}_{1}, \mathbf{u}_{2}\right)\right] \\
=\int_{\substack{\left(\tilde{\mathbf{u}}_{1}, \tilde{\mathbf{u}}_{2}\right) \in \mathcal{S}_{1} \times \mathcal{S}_{2}: \\
\cos \varangle\left(\tilde{\mathbf{u}}_{1}, \tilde{\mathbf{u}}_{2}\right) \geq \Theta,}}^{\cos \varangle\left(\mathbf{y}, \alpha_{1} \tilde{\mathbf{u}}_{1}+\alpha_{2} \tilde{\mathbf{u}}_{2}\right) \geq \Delta} \quad f^{\lambda_{1} \times \lambda_{2}}\left(\left(\mathbf{U}_{1}(2), \mathbf{U}_{2}(2)\right)=\left(\tilde{\mathbf{u}}_{1}, \tilde{\mathbf{u}}_{2}\right) \mid \tilde{\varsigma}\left(\mathbf{S}_{1}, \mathbf{S}_{2}, \mathscr{C}_{1}, \mathscr{C}_{2}\right)=(1,1),\right. \\
\left.\left(\mathbf{S}_{1}, \mathbf{S}_{2}, \mathbf{U}_{1}(1), \mathbf{U}_{2}(1)\right)=\left(\mathbf{s}_{1}, \mathbf{s}_{2}, \mathbf{u}_{1}, \mathbf{u}_{2}\right)\right)\left(\tilde{\mathbf{u}}_{1}, \tilde{\mathbf{u}}_{2}\right)
\end{array}
\end{gathered}
$$




$$
\begin{aligned}
& =\int_{\substack{\left.\cos \varangle\left(\tilde{\mathbf{u}}_{1}, \tilde{\mathbf{u}}_{2}\right) \in \mathcal{S}_{1} \times \mathcal{S}_{2}\right) \geq \Theta, \mathcal{u}_{2}:}} f^{\lambda_{1}}\left(\mathbf{U}_{1}(2)=\tilde{\mathbf{u}}_{1} \mid \tilde{\varsigma}\left(\mathbf{S}_{1}, \mathbf{S}_{2}, \mathscr{C}_{1}, \mathscr{C}_{2}\right)=(1,1),\right. \\
& \left.\cos \varangle\left(\mathbf{y}, \alpha_{1} \tilde{\mathbf{u}}_{1}+\alpha_{2} \tilde{\mathbf{u}}_{2}\right) \geq \Delta \quad\left(\mathbf{S}_{1}, \mathbf{S}_{2}, \mathbf{U}_{1}(1), \mathbf{U}_{2}(1)\right)=\left(\mathbf{s}_{1}, \mathbf{s}_{2}, \mathbf{u}_{1}, \mathbf{u}_{2}\right)\right) \\
& \cdot f^{\lambda_{2}}\left(\mathbf{U}_{2}(2)=\tilde{\mathbf{u}}_{2} \mid \tilde{\varsigma}\left(\mathbf{S}_{1}, \mathbf{S}_{2}, \mathscr{C}_{1}, \mathscr{C}_{2}\right)=(1,1)\right. \\
& \left.\left(\mathbf{S}_{1}, \mathbf{S}_{2}, \mathbf{U}_{1}(1), \mathbf{U}_{2}(1)\right)=\left(\mathbf{s}_{1}, \mathbf{s}_{2}, \mathbf{u}_{1}, \mathbf{u}_{2}\right)\right)\left(\tilde{\mathbf{u}}_{1}, \tilde{\mathbf{u}}_{2}\right) \\
& =\int_{\substack{\cos \varangle\left(\tilde{\mathbf{u}}_{1}, \tilde{\mathbf{u}}_{2}\right) \geq \Theta, \cos \varangle\left(\mathbf{y}, \alpha_{1} \tilde{\mathbf{u}}_{1}+\alpha_{2} \tilde{\mathbf{u}}_{2}\right) \geq \Delta}} f^{\tilde{u}_{1}}\left(\mathbf{U}_{1}(2)=\tilde{\mathbf{u}}_{1} \mid \varsigma_{1}\left(\mathbf{S}_{1}, \mathscr{C}_{1},\right)=1,\left(\mathbf{S}_{1}, \mathbf{U}_{1}(1)\right)=\left(\mathbf{s}_{1}, \mathbf{u}_{1}\right)\right) \\
& \cdot f^{\lambda_{2}}\left(\mathbf{U}_{2}(2)=\tilde{\mathbf{u}}_{2} \mid \varsigma_{2}\left(\mathbf{S}_{2}, \mathscr{C}_{2},\right)=1,\left(\mathbf{S}_{2}, \mathbf{U}_{2}(1)\right)=\left(\mathbf{s}_{2}, \mathbf{u}_{2}\right)\right)\left(\tilde{\mathbf{u}}_{1}, \tilde{\mathbf{u}}_{2}\right) \\
& \stackrel{a)}{\leq} \int_{\substack{\left.\cos \left(\tilde{\mathbf{u}}_{1}, \tilde{\mathbf{u}}_{2}\right) \in \mathcal{S}_{1} \times \mathcal{S}_{2}: \\
\cos \varangle\left(\mathbf{y}, \alpha_{1}, \tilde{\mathbf{u}}_{1}+\alpha_{2}\right) \geq \tilde{\mathbf{u}}_{2}\right) \geq \Delta}} \frac{2}{r_{1}^{n-1} C_{n}(\pi)} \cdot \frac{2}{r_{2}^{n-1} C_{n}(\pi)} \cdot\left(\tilde{\mathbf{u}}_{1}, \tilde{\mathbf{u}}_{2}\right) \\
& \stackrel{b)}{=} 4 \frac{C_{n}(\arccos \Theta)}{C_{n}(\pi)} \cdot \frac{C_{n}(\arccos \Delta)}{C_{n}(\pi)},
\end{aligned}
$$

where in $a$ ) we have used Lemma E.19 and in $b$ ) we have used that under distributions of $\mathbf{U}_{1}(2)$ and $\mathbf{U}_{2}(2)$ that are independent of $\mathbf{y}$ and uniform over $\mathcal{S}_{1}$ and $\mathcal{S}_{2}$ respectively, the angles $\varangle\left(\mathbf{U}_{1}(j), \mathbf{U}_{2}(\ell)\right)$ and $\varangle\left(\mathbf{y}, \alpha_{1} \mathbf{U}_{1}(j)+\alpha_{2} \mathbf{U}_{2}(\ell)\right)$ are independent. Thus, combining (148) with (147) gives

$$
\operatorname{Pr}\left[\mathcal{G} \mid \mathcal{E}_{\mathbf{X}_{1}}^{c} \cap \mathcal{E}_{\mathbf{X}_{2}}^{c}\right]<2^{n\left(R_{1}+R_{2}\right)} \cdot 4 \frac{C_{n}(\arccos \Theta)}{C_{n}(\pi)} \cdot \frac{C_{n}(\arccos \Delta)}{C_{n}(\pi)} .
$$

And combining (149) with Lemma E.17 gives

$$
\begin{aligned}
\operatorname{Pr} & {\left[\mathcal{G} \mid \mathcal{E}_{\mathbf{X}_{1}}^{c} \cap \mathcal{E}_{\mathbf{X}_{2}}^{c}\right] } \\
& <2^{n\left(R_{1}+R_{2}\right)} \cdot 4 \frac{\Gamma\left(\frac{n}{2}+1\right)\left(1-\Theta^{2}\right)^{(n-1) / 2}}{n \Gamma\left(\frac{n+1}{2}\right) \sqrt{\pi} \Theta} \cdot \frac{\Gamma\left(\frac{n}{2}+1\right)\left(1-\Delta^{2}\right)^{(n-1) / 2}}{n \Gamma\left(\frac{n+1}{2}\right) \sqrt{\pi} \Delta} \\
& =4 \cdot\left(\frac{\Gamma\left(\frac{n}{2}+1\right)}{n \Gamma\left(\frac{n+1}{2}\right) \sqrt{\pi}}\right)^{2} \frac{1}{\Theta \sqrt{1-\Theta^{2}} \Delta \sqrt{1-\Delta^{2}}} 2^{n\left(R_{1}+R_{2}+\frac{1}{2} \log _{2}\left(\left(1-\Theta^{2}\right)\left(1-\Delta^{2}\right)\right)\right)}
\end{aligned}
$$

Replacing the ratios of the Gamma-functions by their asymptotic series in Lemma E.18 finally establishes (117).

\section{F Proof of Theorem 4.5}

The high-SNR asymptotics for the multiple-access problem without feedback can be obtained from the necessary condition for the achievability of a distortion pair $\left(D_{1}, D_{2}\right)$ 
in Theorem 4.1, and from the sufficient conditions for the achievability of a distortion pair $\left(D_{1}, D_{2}\right)$ deriving from the vector-quantizer scheme in Theorem 4.4.

By Theorem 4.4 it follows that any distortion pair $\left(\bar{D}_{1}, \bar{D}_{2}\right)$ satisfying $\bar{D}_{1} \leq \sigma^{2}$, $\bar{D}_{2} \leq \sigma^{2}$ and

$$
\begin{aligned}
\bar{D}_{1} & \geq \sigma^{2} \frac{N}{P_{1}} \\
\bar{D}_{2} & \geq \sigma^{2} \frac{N}{P_{2}} \\
\bar{D}_{1} \bar{D}_{2} & =\sigma^{4} \frac{N\left(1-\breve{\rho}^{2}\right)}{P_{1}+P_{2}+2 \breve{\rho} \sqrt{P_{1} P_{2}}}
\end{aligned}
$$

where

$$
\breve{\rho}=\rho \sqrt{\left(1-\frac{\bar{D}_{1}}{\sigma^{2}}\right)\left(1-\frac{\bar{D}_{2}}{\sigma^{2}}\right)},
$$

is achievable. If

$$
\lim _{N \rightarrow 0} \frac{N}{P_{1} \bar{D}_{1}}=0 \quad \text { and } \quad \lim _{N \rightarrow 0} \frac{N}{P_{2} \bar{D}_{2}}=0,
$$

then for $N$ sufficiently small, (150) and (151) are satisfied. Consequently, for $N$ sufficiently small any pair satisfying (152) and (154) is achievable. We next show that if the pair $\left(\bar{D}_{1}, \bar{D}_{2}\right)$ satisfies (152) and (154), then $\breve{\rho} \rightarrow \rho$ as $N \rightarrow 0$. To show this, we note that if $\left(\bar{D}_{1}, \bar{D}_{2}\right)$ satisfies (152) then

$$
\bar{D}_{2} \leq \sigma^{4} \frac{N}{P_{1} \bar{D}_{1}}, \quad \text { and } \quad \bar{D}_{1} \leq \sigma^{4} \frac{N}{P_{2} \bar{D}_{2}} .
$$

Combining (155) with (153) gives that if in addition to (152) the pair $\left(\bar{D}_{1}, \bar{D}_{2}\right)$ also satisfies (155), then $\breve{\rho} \rightarrow \rho$ as $N \rightarrow 0$. Thus, if $\left(\bar{D}_{1}, \bar{D}_{2}\right)$ satisfies (152) and (154), then

$$
\lim _{N \rightarrow 0} \frac{P_{1}+P_{2}+2 \rho \sqrt{P_{1} P_{2}}}{N} \bar{D}_{1} \bar{D}_{2} \leq \sigma^{4}\left(1-\rho^{2}\right) \text {. }
$$

Now, let $\left(D_{1}^{*}\left(\sigma^{2}, \rho, P_{1}, P_{2}, N\right), D_{2}^{*}\left(\sigma^{2}, \rho, P_{1}, P_{2}, N\right)\right)$ be a distortion pair resulting from an optimal scheme and let $\left(D_{1}^{*}, D_{2}^{*}\right)$ be the shorthand notation for this distortion pair. By Theorem 4.1 we have that

$$
R_{S_{1}, S_{2}}\left(D_{1}^{*}, D_{2}^{*}\right) \leq \frac{1}{2} \log _{2}\left(1+\frac{P_{1}+P_{2}+2 \rho \sqrt{P_{1} P_{2}}}{N}\right) .
$$

If $\left(D_{1}^{*}, D_{2}^{*}\right)$ satisfies

$$
\lim _{N \rightarrow 0} \frac{N}{P_{1} D_{1}^{*}}=0 \quad \text { and } \quad \lim _{N \rightarrow 0} \frac{N}{P_{2} D_{2}^{*}}=0,
$$

then for $N$ sufficiently small

$$
R_{S_{1}, S_{2}}\left(D_{1}^{*}, D_{2}^{*}\right)=\frac{1}{2} \log _{2}^{+}\left(\frac{\sigma^{4}\left(1-\rho^{2}\right)}{D_{1}^{*} D_{2}^{*}}\right),
$$

by Theorem 3.1 and because $\left(D_{1}^{*}, D_{2}^{*}\right) \in \mathscr{D}_{2}$. From (157) and (159) we thus get that if $\left(D_{1}^{*}, D_{2}^{*}\right)$ satisfies (158), then

$$
\lim _{N \rightarrow 0} \frac{P_{1}+P_{2}+2 \rho \sqrt{P_{1} P_{2}}}{N} D_{1}^{*} D_{2}^{*} \geq \sigma^{4}\left(1-\rho^{2}\right) .
$$

Combining (156) with (160) yields Theorem 4.5. 


\section{G Proof of Theorem 4.6}

Our analysis of the expected distortion for the superimposed scheme is based on a genie-aided argument, similar as in the analysis of the vector-quantizer scheme. This argument is described more precisely now.

\section{G.1 Genie-Aided Scheme}

In our genie-aided argument, the genie assists the decoder. An illustration of this decoder is given in Figure 13, In addition to the channel output $\mathbf{Y}$ that is observed orig-

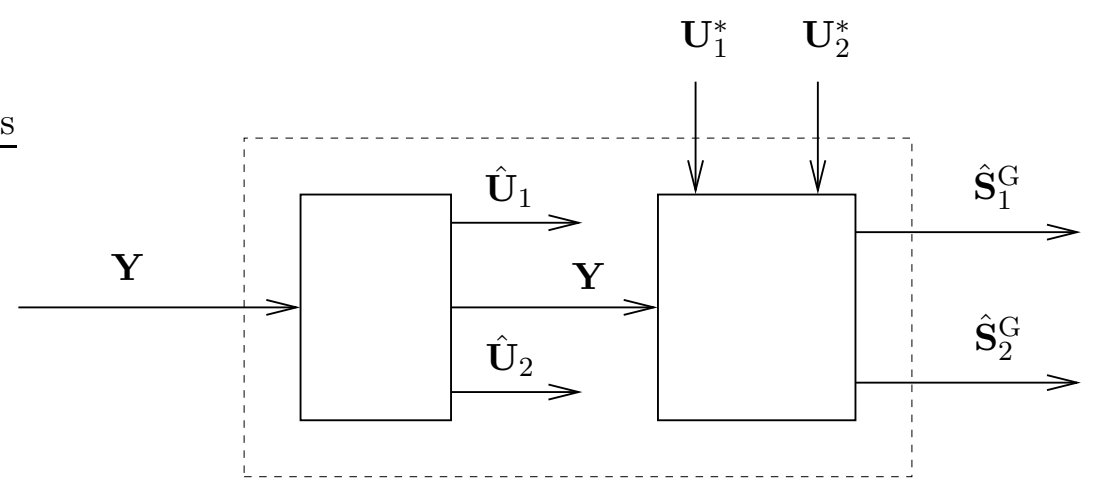

Figure 13: Genie-aided decoder.

inally, the decoder is now also provided with the transmitted codeword pair $\left(\mathbf{U}_{1}^{*}, \mathbf{U}_{2}^{*}\right)$. Based on $\left(\mathbf{U}_{1}^{*}, \mathbf{U}_{2}^{*}\right)$ and $\mathbf{Y}$, the decoder then estimates the source pair $\left(\mathbf{S}_{1}, \mathbf{S}_{2}\right)$ and thereby ignores the guess $\left(\hat{\mathbf{U}}_{1}, \hat{\mathbf{U}}_{2}\right)$ produced in the first step of the original decoder. The estimate of this genie-aided decoder is denoted by $\left(\hat{\mathbf{S}}_{1}^{\mathrm{G}}, \hat{\mathbf{S}}_{2}^{\mathrm{G}}\right)$ and is given by

$$
\begin{aligned}
& \hat{\mathbf{S}}_{1}^{\mathrm{G}}=\gamma_{11} \mathbf{U}_{1}^{*}+\gamma_{12} \mathbf{U}_{2}^{*}+\gamma_{13} \mathbf{Y} \\
& \hat{\mathbf{S}}_{2}^{\mathrm{G}}=\gamma_{21} \mathbf{U}_{2}^{*}+\gamma_{22} \mathbf{U}_{1}^{*}+\gamma_{23} \mathbf{Y},
\end{aligned}
$$

where the coefficients $\gamma_{i j}$ are as defined in (33). We now show that under certain rate constraints, the normalized asymptotic distortion of this genie-aided scheme is the same as for the originally proposed scheme. The key argument is stated in the following proposition.

Proposition G.1. For every $\delta>0$ and $0<\epsilon<0.3$ there exists an $n^{\prime}(\delta, \epsilon)>0$ such that for all $n>n^{\prime}(\delta, \epsilon)$,

$$
\frac{1}{n} \mathrm{E}\left[\left\|\mathbf{S}_{1}-\hat{\mathbf{S}}_{1}\right\|^{2}\right] \leq \frac{1}{n} \mathrm{E}\left[\left\|\mathbf{S}_{1}-\hat{\mathbf{S}}_{1}^{\mathrm{G}}\right\|^{2}\right]+\xi_{1}^{\prime} \delta+\xi_{2}^{\prime} \epsilon,
$$

whenever $\left(R_{1}, R_{2}\right)$ is in the rate region $\mathcal{R}^{\prime}(\epsilon)$ given by

$$
\begin{aligned}
& \mathcal{R}^{\prime}(\epsilon)=\left\{\quad R_{1} \leq \frac{1}{2} \log _{2}\left(\frac{\beta_{1}^{\prime 2}\left\|\mathbf{U}_{1}\right\|^{2}\left(1-\tilde{\rho}^{2}\right)+N^{\prime}}{N^{\prime}\left(1-\tilde{\rho}^{2}\right)}-\kappa_{1} \epsilon\right),\right. \\
& R_{2} \leq \frac{1}{2} \log _{2}\left(\frac{\beta_{2}^{\prime 2}\left\|\mathbf{U}_{2}\right\|^{2}\left(1-\tilde{\rho}^{2}\right)+N^{\prime}}{N^{\prime}\left(1-\tilde{\rho}^{2}\right)}-\kappa_{2} \epsilon\right),
\end{aligned}
$$




$$
\left.R_{1}+R_{2} \leq \frac{1}{2} \log _{2}\left(\frac{\beta_{1}^{\prime 2}\left\|\mathbf{U}_{1}\right\|^{2}+\beta_{1}^{\prime 2}\left\|\mathbf{U}_{1}\right\|^{2}+2 \tilde{\rho} \beta_{1}^{\prime} \beta_{2}^{\prime}\left\|\mathbf{U}_{1}\right\|\left\|\mathbf{U}_{2}\right\|+N^{\prime}}{N^{\prime}\left(1-\tilde{\rho}^{2}\right)}-\kappa_{3} \epsilon\right)\right\},
$$

where in (163) $\xi_{1}^{\prime}$ and $\xi_{2}^{\prime}$ depend only on $\sigma^{2}, \gamma_{13}, P_{1}, P_{2}$ and $N$, and where in the expression of $\mathcal{R}^{\prime}(\epsilon)$ the terms $\kappa_{1}, \kappa_{2}$ and $\kappa_{3}$ depend only on $P_{1}, P_{2}, N^{\prime}$, and $\tilde{\rho}$, and where $N^{\prime}$ and $\beta_{1}^{\prime}, \beta_{2}^{\prime}$ are as given in (34), (35) and (36) respectively.

Proof. See Section G.2.

From Proposition G.1 it now follows easily that the expected distortion asymptotically achievable by the genie-aided scheme is the same as the expected distortion achievable by the original scheme.

Corollary G.1. If $\left(R_{1}, R_{2}\right)$ satisfy

$$
\begin{aligned}
R_{1} & <\frac{1}{2} \log _{2}\left(\frac{\beta_{1}^{\prime 2}\left\|\mathbf{U}_{1}\right\|^{2}\left(1-\tilde{\rho}^{2}\right)+N^{\prime}}{N^{\prime}\left(1-\tilde{\rho}^{2}\right)}\right), \\
R_{2} & <\frac{1}{2} \log _{2}\left(\frac{\beta_{2}^{\prime 2}\left\|\mathbf{U}_{2}\right\|^{2}\left(1-\tilde{\rho}^{2}\right)+N^{\prime}}{N^{\prime}\left(1-\tilde{\rho}^{2}\right)}\right), \\
R_{1}+R_{2} & <\frac{1}{2} \log _{2}\left(\frac{\beta_{1}^{\prime 2}\left\|\mathbf{U}_{1}\right\|^{2}+\beta_{1}^{\prime 2}\left\|\mathbf{U}_{1}\right\|^{2}+2 \tilde{\rho} \beta_{1}^{\prime} \beta_{2}^{\prime}\left\|\mathbf{U}_{1}\right\|\left\|\mathbf{U}_{2}\right\|+N^{\prime}}{N^{\prime}\left(1-\tilde{\rho}^{2}\right)}\right),
\end{aligned}
$$

then

$$
\varlimsup_{n \rightarrow \infty} \frac{1}{n} \mathrm{E}\left[\left\|\mathbf{S}_{1}-\hat{\mathbf{S}}_{1}\right\|^{2}\right] \leq \varlimsup_{n \rightarrow \infty} \frac{1}{n} \mathrm{E}\left[\left\|\mathbf{S}_{1}-\hat{\mathbf{S}}_{1}^{\mathrm{G}}\right\|^{2}\right] .
$$

Proof. Follows from Proposition G.1 by first letting $n \rightarrow \infty$ and then $\epsilon \rightarrow 0$ and $\delta \rightarrow 0$.

It follows by Corollary G.1 that to analyze the distortion achievable by our scheme it suffices to analyze the genie-aided scheme. This is done in Section G.3.

\section{G.2 Proof of Proposition G.1}

The proof of Proposition G.1 consists of upper bounding the difference between $\frac{1}{n} E\left[\left\|\mathbf{S}_{1}-\hat{\mathbf{S}}_{1}\right\|^{2}\right]$ and $\frac{1}{n} E\left[\left\|\mathbf{S}_{1}-\hat{\mathbf{S}}_{1}^{\mathrm{G}}\right\|^{2}\right]$. Since the two estimates $\hat{\mathbf{S}}_{1}$ and $\hat{\mathbf{S}}_{1}^{\mathrm{G}}$ differ only if $\left(\hat{\mathbf{U}}_{1}, \hat{\mathbf{U}}_{2}\right) \neq$ $\left(\mathbf{U}_{1}^{*}, \mathbf{U}_{2}^{*}\right)$, the main step is to upper bound the probability of a decoding error. This is what we do now.

Let the error event $\mathcal{E}_{\hat{\mathbf{U}}}$ be as defined in (89) - (91) for the vector-quantizer scheme. The probability of $\mathcal{E}_{\hat{\mathbf{U}}}$ is upper bounded in the following lemma.

Lemma G.1. For every $\delta>0$ and $0<\epsilon<0.3$, there exists an $n_{4}^{\prime}(\delta, \epsilon) \in \mathbb{N}$ such that for all $n>n_{4}^{\prime}(\delta, \epsilon)$

$$
\operatorname{Pr}\left[\mathcal{E}_{\hat{\mathbf{U}}}\right]<11 \delta \quad \text { whenever }\left(R_{1}, R_{2}\right) \in \mathcal{R}^{\prime}(\epsilon) .
$$


Proof. The proof follows from restating the decoding problem for the superimposed scheme in the form of the decoding problem for the vector-quantizer scheme. That is, we seek to rewrite the channel output in the form

$$
\mathbf{Y}=\beta_{1}^{\prime} \mathbf{U}_{1}^{*}+\beta_{2}^{\prime} \mathbf{U}_{2}^{*}+\mathbf{Z}^{\prime}
$$

with an additive noise sequence $\mathbf{Z}^{\prime}$ that satisfies the properties needed for the analysis of the vector-quantizer scheme. This representation is obtained by first rewriting the source sequences as

$$
\begin{aligned}
& \mathbf{S}_{1}=\left(1-a_{1} \tilde{\rho}\right) \mathbf{U}_{1}^{*}+a_{1} \mathbf{U}_{2}^{*}+\mathbf{W}_{1} \\
& \mathbf{S}_{2}=\left(1-a_{2} \tilde{\rho}\right) \mathbf{U}_{2}^{*}+a_{2} \mathbf{U}_{1}^{*}+\mathbf{W}_{2},
\end{aligned}
$$

where $a_{1}$ is defined in (37), $a_{2}$ is defined in (38), and $\tilde{\rho}$ is defined in (26). Combining (165) and (166) with the expressions for $\mathbf{X}_{1}$ and $\mathbf{X}_{2}$ in (29) and with $\mathbf{Y}=\mathbf{X}_{1}+\mathbf{X}_{2}+\mathbf{Z}$ yields the desired form of (164) with

$$
\begin{aligned}
& \beta_{1}^{\prime}=\left(\alpha_{1}\left(1-a_{1} \tilde{\rho}\right)+\beta_{1}+\alpha_{2} a_{2}\right) \\
& \beta_{2}^{\prime}=\left(\alpha_{2}\left(1-a_{2} \tilde{\rho}\right)+\beta_{2}+\alpha_{1} a_{1}\right),
\end{aligned}
$$

and with

$$
\mathbf{Z}^{\prime}=\alpha_{1} \mathbf{W}_{1}+\alpha_{2} \mathbf{W}_{2}+\mathbf{Z}
$$

For the additive noise sequence $\mathbf{Z}^{\prime}$ it can now be verified that for every $\delta>0$ and $\epsilon>0$ there exists an $n^{\prime}(\delta, \epsilon)>0$, such that for $N^{\prime}$ as in (34) and for all $n>n^{\prime}(\delta, \epsilon)$ we have that

$$
\operatorname{Pr}\left[\left|\frac{1}{n}\left\|\mathbf{Z}^{\prime}\right\|^{2}-N^{\prime}\right| \leq N^{\prime} \epsilon\right]>1-\delta
$$

and that

$$
\operatorname{Pr}\left[\left|\left\langle\mathbf{U}_{i}^{*}, \mathbf{Z}^{\prime}\right\rangle\right| \leq n \sqrt{\sigma^{2}\left(1-2^{-2 R_{i}}\right) N^{\prime}} \epsilon\right]>1-\delta, \quad i \in\{1,2\} .
$$

Condition (168) follows since for $a_{1}$ and $a_{2}$, given in (37) and (38), for sufficiently large $n$, we have with high probability that

$$
\left\langle\mathbf{U}_{i}^{*}, \mathbf{W}_{j}\right\rangle \approx 0 \quad \forall i, j \in\{1,2\} .
$$

Conditions (167) and (168) are precisely those needed in the proof of the achievable rates for the vector-quantizer scheme. Hence, the upper bound on the probability of a decoding error in the vector-quantizer scheme given in Lemma E.1 can be adopted to the superimposed scheme. This yields Lemma G.1,

To ease the upper bounding of the difference between $\frac{1}{n} E\left[\left\|\mathbf{S}_{1}-\hat{\mathbf{S}}_{1}\right\|^{2}\right]$ and $\frac{1}{n} E\left[\| \mathbf{S}_{1}-\right.$ $\left.\hat{\mathbf{S}}_{1}^{\mathrm{G}} \|^{2}\right]$ we now state three more lemmas which upper bound different norms and inner products involving $\mathbf{S}_{1}, \hat{\mathbf{S}}_{1}$ and $\hat{\mathbf{S}}_{1}^{\mathrm{G}}$. The first lemma gives an upper bound on the squared norm of $\hat{\mathbf{S}}_{1}-\hat{\mathbf{S}}_{1}^{\mathrm{G}}$. 
Lemma G.2. Let the reconstructions $\hat{\mathbf{S}}_{1}$ and $\hat{\mathbf{S}}_{1}^{\mathrm{G}}$ be as defined in (31) and (161). Then, with probability one

$$
\left\|\hat{\mathbf{S}}_{1}-\hat{\mathbf{S}}_{1}^{\mathrm{G}}\right\|^{2} \leq 16 n \sigma^{2}
$$

Proof.

$$
\begin{aligned}
\left\|\hat{\mathbf{S}}_{1}-\hat{\mathbf{S}}_{1}^{\mathrm{G}}\right\|^{2}= & \left\|\gamma_{11}\left(\hat{\mathbf{U}}_{1}-\mathbf{U}_{1}^{*}\right)+\gamma_{12}\left(\hat{\mathbf{U}}_{2}-\mathbf{U}_{2}^{*}\right)\right\|^{2} \\
= & \gamma_{11}^{2}\left\|\hat{\mathbf{U}}_{1}-\mathbf{U}_{1}^{*}\right\|^{2}+2 \gamma_{11} \gamma_{12}\left\langle\hat{\mathbf{U}}_{1}-\mathbf{U}_{1}^{*}, \hat{\mathbf{U}}_{2}-\mathbf{U}_{2}^{*}\right\rangle \\
& +\gamma_{12}^{2}\left\|\hat{\mathbf{U}}_{2}-\mathbf{U}_{2}^{*}\right\|^{2} \\
\leq & \gamma_{11}^{2} \underbrace{\left\|\hat{\mathbf{U}}_{1}-\mathbf{U}_{1}^{*}\right\|^{2}}_{\leq 4 n \sigma^{2}}+2 \gamma_{11} \gamma_{12} \underbrace{\left\|\hat{\mathbf{U}}_{1}-\mathbf{U}_{1}^{*}\right\|\left\|\hat{\mathbf{U}}_{2}-\mathbf{U}_{2}^{*}\right\|}_{\leq 4 n \sigma^{2}} \\
& +\gamma_{12}^{2} \underbrace{\left\|\hat{\mathbf{U}}_{2}-\mathbf{U}_{2}^{*}\right\|^{2}}_{\leq 4 n \sigma^{2}} \\
\leq & 4 n \sigma^{2}\left(\gamma_{11}+\gamma_{12}\right)^{2} \\
\leq & 16 n \sigma^{2},
\end{aligned}
$$

where in the last step we have used that $0 \leq \gamma_{11}, \gamma_{12} \leq 1$.

For the next two lemmas, we reuse the two error events $\mathcal{E}_{\mathbf{S}}$ and $\mathcal{E}_{\mathbf{Z}}$ which were defined in (92) and (93) for the proof of the vector-quantizer scheme. We then have:

Lemma G.3. For every $\epsilon>0$

$$
\frac{1}{n} \mathrm{E}\left[\left\langle\mathbf{S}_{1}, \hat{\mathbf{S}}_{1}^{\mathrm{G}}-\hat{\mathbf{S}}_{1}\right\rangle\right] \leq \sigma^{2}\left(\epsilon+17 \operatorname{Pr}\left[\mathcal{E}_{\mathbf{S}}\right]+(17+\epsilon) \operatorname{Pr}\left[\mathcal{E}_{\hat{\mathbf{U}}}\right]\right) .
$$

Proof.

$$
\begin{aligned}
\frac{1}{n} \mathrm{E}\left[\left\langle\mathbf{S}_{1}, \hat{\mathbf{S}}_{1}^{\mathrm{G}}-\hat{\mathbf{S}}_{1}\right\rangle\right]= & \frac{1}{n} \mathrm{E}\left[\left\langle\mathbf{S}_{1}, \hat{\mathbf{S}}_{1}^{\mathrm{G}}-\hat{\mathbf{S}}_{1}\right\rangle \mid \mathcal{E}_{\mathbf{S}} \cup \mathcal{E}_{\hat{\mathbf{U}}}\right] \operatorname{Pr}\left[\mathcal{E}_{\mathbf{S}} \cup \mathcal{E}_{\hat{\mathbf{U}}}\right] \\
& +\frac{1}{n} \underbrace{\mathrm{E}\left[\left\langle\mathbf{S}_{1}, \hat{\mathbf{S}}_{1}^{\mathrm{G}}-\hat{\mathbf{S}}_{1}\right\rangle \mid \mathcal{E}_{\mathbf{S}}^{c} \cap \mathcal{E}_{\hat{\mathbf{U}}}^{c}\right]}_{=0} \operatorname{Pr}\left[\mathcal{E}_{\mathbf{S}}^{c} \cap \mathcal{E}_{\hat{\mathbf{U}}}^{c}\right] \\
\leq a) & \frac{1}{n} \mathrm{E}\left[\left\|\mathbf{S}_{1}\right\|^{2}+\left\|\hat{\mathbf{S}}_{1}^{\mathrm{G}}-\hat{\mathbf{S}}_{1}\right\|^{2} \mid \mathcal{E}_{\mathbf{S}} \cup \mathcal{E}_{\hat{\mathbf{U}}}\right] \operatorname{Pr}\left[\mathcal{E}_{\mathbf{S}} \cup \mathcal{E}_{\hat{\mathbf{U}}}\right] \\
= & \frac{1}{n} \mathrm{E}\left[\left\|\mathbf{S}_{1}\right\|^{2} \mid \mathcal{E}_{\mathbf{S}}\right] \operatorname{Pr}\left[\mathcal{E}_{\mathbf{S}}\right]+\frac{1}{n} \mathrm{E}\left[\left\|\mathbf{S}_{1}\right\|^{2} \mid \mathcal{E}_{\mathbf{S}}^{c} \cap \mathcal{E}_{\hat{\mathbf{U}}}\right] \operatorname{Pr}\left[\mathcal{E}_{\mathbf{S}}^{c} \cap \mathcal{E}_{\hat{\mathbf{U}}}\right] \\
& +\frac{1}{n} \mathrm{E}\left[\left\|\hat{\mathbf{S}}_{1}^{\mathrm{G}}-\hat{\mathbf{S}}_{1}\right\|^{2} \mid \mathcal{E}_{\mathbf{S}} \cup \mathcal{E}_{\hat{\mathbf{U}}}\right] \operatorname{Pr}\left[\mathcal{E}_{\mathbf{S}} \cup \mathcal{E}_{\hat{\mathbf{U}}}\right] \\
& b) \\
\leq & \sigma^{2}\left(\epsilon+\operatorname{Pr}\left[\mathcal{E}_{\mathbf{S}}\right]\right)+\sigma^{2}(1+\epsilon) \operatorname{Pr}\left[\mathcal{E}_{\hat{\mathbf{U}}}\right] \\
& +16 \sigma^{2}\left(\operatorname{Pr}\left[\mathcal{E}_{\mathbf{S}}\right]+\operatorname{Pr}\left[\mathcal{E}_{\hat{\mathbf{U}}}\right]\right) \\
\leq & \sigma^{2}\left(\epsilon+17 \operatorname{Pr}\left[\mathcal{E}_{\mathbf{S}}\right]+(17+\epsilon) \operatorname{Pr}\left[\mathcal{E}_{\hat{\mathbf{U}}}\right]\right)
\end{aligned}
$$


where in the first equality, the second expectation term equals zero because by $\mathcal{E}_{\hat{\mathbf{U}}}^{c}$ we have $\left\|\hat{\mathbf{s}}_{1}^{\mathrm{G}}-\hat{\mathbf{s}}_{1}\right\|=0$ and by $\mathcal{E}_{\mathbf{S}}^{c}$ the norm $\left\|\mathbf{s}_{1}\right\|$ is bounded. In $a$ ) we have used (121), and in $b$ ) we have used Lemma E.10. Lemma G.2 and the fact that conditioned on $\mathcal{E}_{\mathrm{S}}^{c}$ we have $\left\|\mathbf{s}_{1}\right\|^{2} \leq n \sigma^{2}(1+\epsilon)$.

Lemma G.4. For every $\epsilon>0$

$$
\begin{aligned}
\frac{1}{n} \mathrm{E}\left[\left\|\hat{\mathbf{S}}_{1}\right\|^{2}-\left\|\hat{\mathbf{S}}_{1}^{\mathrm{G}}\right\|^{2}\right] \leq & 4\left(\sigma^{2}\left(1+4 \gamma_{13}\right)+9 \gamma_{13}\left(P_{1}+P_{2}+N\right)(1+\epsilon)\right) \operatorname{Pr}\left[\mathcal{E}_{\hat{\mathbf{U}}}\right] \\
& +36 \gamma_{13}\left(\left(P_{1}+P_{2}\right) \operatorname{Pr}\left[\mathcal{E}_{\mathbf{S}}\right]+N \operatorname{Pr}\left[\mathcal{E}_{\mathbf{Z}}\right]+\left(P_{1}+P_{2}+N\right) \epsilon\right) .
\end{aligned}
$$

Proof.

$$
\begin{aligned}
\frac{1}{n} \mathrm{E}\left[\left\|\hat{\mathbf{S}}_{1}\right\|^{2}-\left\|\hat{\mathbf{S}}_{1}^{\mathrm{G}}\right\|^{2}\right]= & \frac{1}{n} \mathrm{E}\left[\left\|\hat{\mathbf{S}}_{1}\right\|^{2}-\left\|\hat{\mathbf{S}}_{1}^{\mathrm{G}}\right\|^{2} \mid \mathcal{E}_{\hat{\mathbf{U}}}\right] \operatorname{Pr}\left[\mathcal{E}_{\hat{\mathbf{U}}}\right] \\
& +\frac{1}{n} \mathrm{E}\left[\left\|\hat{\mathbf{S}}_{1}\right\|^{2}-\left\|\hat{\mathbf{S}}_{1}^{\mathrm{G}}\right\|^{2} \mid \mathcal{E}_{\hat{\mathbf{U}}}^{c}\right] \operatorname{Pr}\left[\mathcal{E}_{\hat{\mathbf{U}}}^{c}\right] \\
\leq & \frac{1}{n} \mathrm{E}\left[\left\|\hat{\mathbf{S}}_{1}\right\|^{2}-\left\|\hat{\mathbf{S}}_{1}^{\mathrm{G}}\right\|^{2} \mid \mathcal{E}_{\hat{\mathbf{U}}}\right] \operatorname{Pr}\left[\mathcal{E}_{\hat{\mathbf{U}}}\right],
\end{aligned}
$$

where the last inequality follows since conditional on $\mathcal{E}_{\hat{\mathbf{U}}}^{c}$ we have $\hat{\mathbf{S}}_{1}=\hat{\mathbf{S}}_{1}^{G}$ and therefore $\left\|\hat{\mathbf{S}}_{1}\right\|^{2}-\left\|\hat{\mathbf{S}}_{1}^{\mathrm{G}}\right\|^{2}=0$. To upper bound the RHS of (170), we now upper bound the difference $\left\|\hat{\mathbf{S}}_{1}\right\|^{2}-\left\|\hat{\mathbf{S}}_{1}^{\mathrm{G}}\right\|^{2}$ :

$$
\begin{aligned}
\left\|\hat{\mathbf{S}}_{1}\right\|^{2}-\left\|\hat{\mathbf{S}}_{1}^{\mathrm{G}}\right\|^{2}= & \gamma_{11}^{2}\left\|\hat{\mathbf{U}}_{1}\right\|^{2}+2 \gamma_{11} \gamma_{12}\left\langle\hat{\mathbf{U}}_{1}, \hat{\mathbf{U}}_{2}\right\rangle+2 \gamma_{11} \gamma_{13}\left\langle\hat{\mathbf{U}}_{1}, \mathbf{Y}\right\rangle \\
& +\gamma_{12}^{2}\left\|\hat{\mathbf{U}}_{2}\right\|^{2}+2 \gamma_{12} \gamma_{13}\left\langle\hat{\mathbf{U}}_{2}, \mathbf{Y}\right\rangle+\gamma_{13}^{2}\|\mathbf{Y}\|^{2} \\
& -\gamma_{11}^{2}\left\|\mathbf{U}_{1}^{*}\right\|^{2}-2 \gamma_{11} \gamma_{12}\left\langle\mathbf{U}_{1}^{*}, \mathbf{U}_{2}^{*}\right\rangle-2 \gamma_{11} \gamma_{13}\left\langle\mathbf{U}_{1}^{*}, \mathbf{Y}\right\rangle \\
& -\gamma_{12}^{2}\left\|\mathbf{U}_{2}^{*}\right\|^{2}-2 \gamma_{12} \gamma_{13}\left\langle\mathbf{U}_{2}^{*}, \mathbf{Y}\right\rangle-\gamma_{13}^{2}\|\mathbf{Y}\|^{2} \\
= & 2 \gamma_{11} \gamma_{12} \underbrace{\left\langle\hat{\mathbf{U}}_{1}, \hat{\mathbf{U}}_{2}\right\rangle}_{\leq n \sigma^{2}}+2 \gamma_{11} \gamma_{13}\left\langle\hat{\mathbf{U}}_{1}, \mathbf{Y}\right\rangle+2 \gamma_{12} \gamma_{13}\left\langle\hat{\mathbf{U}}_{2}, \mathbf{Y}\right\rangle \\
& -2 \gamma_{11} \gamma_{12} \underbrace{\left\langle\mathbf{U}_{1}^{*}, \mathbf{U}_{2}^{*}\right\rangle}_{\geq-n \sigma^{2}}-2 \gamma_{11} \gamma_{13}\left\langle\mathbf{U}_{1}^{*}, \mathbf{Y}\right\rangle-2 \gamma_{12} \gamma_{13}\left\langle\mathbf{U}_{2}^{*}, \mathbf{Y}\right\rangle \\
\leq & 4 \gamma_{11} \gamma_{12} n \sigma^{2}+2 \gamma_{11} \gamma_{13}\left\langle\hat{\mathbf{U}}_{1}-\mathbf{U}_{1}^{*}, \mathbf{Y}\right\rangle \\
& +2 \gamma_{12} \gamma_{13}\left\langle\hat{\mathbf{U}}_{2}-\mathbf{U}_{2}^{*}, \mathbf{Y}\right\rangle \\
a) & 4 \gamma_{11} \gamma_{12} n \sigma^{2}+2 \gamma_{11} \gamma_{13}(\underbrace{\left\|\hat{\mathbf{U}}_{1}-\mathbf{U}_{1}^{*}\right\|^{2}}_{\leq 4 n \sigma^{2}}+\|\mathbf{Y}\|^{2}) \\
& +2 \gamma_{12} \gamma_{13}(\underbrace{\left\|\hat{\mathbf{U}}_{2}-\mathbf{U}_{2}^{*}\right\|^{2}}_{\leq 4 n \sigma^{2}}+\|\mathbf{Y}\|^{2}) \\
\leq & 4 \gamma_{11} \gamma_{12} n \sigma^{2}+2 \gamma_{13}\left(\gamma_{11}+\gamma_{12}\right)\left(4 n \sigma^{2}+\|\mathbf{Y}\|^{2}\right) \\
\leq & 4 n \sigma^{2}+4 \gamma_{13}\left(4 n \sigma^{2}+\|\mathbf{Y}\|^{2}\right),
\end{aligned}
$$


where in $a$ ) we have used (121), and in the last inequality we have used that $0 \leq$ $\gamma_{11}, \gamma_{12} \leq 1$. We now upper bound the squared norm of $\mathbf{Y}$ on the RHS of (171) in terms of $\mathbf{S}_{1}, \mathbf{S}_{2}, \mathbf{U}_{1}^{*}, \mathbf{U}_{2}^{*}$ and $\mathbf{Z}$ :

$$
\begin{aligned}
\|\mathbf{Y}\|^{2} \leq & \alpha_{1}^{2}\left\|\mathbf{S}_{1}\right\|^{2}+2\left\langle\alpha_{1} \mathbf{S}_{1}, \beta_{1} \mathbf{U}_{1}^{*}\right\rangle+2\left\langle\alpha_{1} \mathbf{S}_{1}, \alpha_{2} \mathbf{S}_{2}\right\rangle+2\left\langle\alpha_{1} \mathbf{S}_{1}, \beta_{2} \mathbf{U}_{2}^{*}\right\rangle \\
& +2\left\langle\alpha_{1} \mathbf{S}_{1}, \mathbf{Z}\right\rangle+\beta_{1}^{2}\left\|\mathbf{U}_{1}^{*}\right\|^{2}+2\left\langle\beta_{1} \mathbf{U}_{1}^{*}, \alpha_{2} \mathbf{S}_{2}\right\rangle+2\left\langle\beta_{1} \mathbf{U}_{1}^{*}, \beta_{2} \mathbf{U}_{2}^{*}\right\rangle \\
& +2\left\langle\beta_{1} \mathbf{U}_{1}^{*}, \mathbf{Z}\right\rangle+\alpha_{2}^{2}\left\|\mathbf{S}_{2}\right\|^{2}+2\left\langle\alpha_{2} \mathbf{S}_{2}, \beta_{2} \mathbf{U}_{2}^{*}\right\rangle+2\left\langle\alpha_{2} \mathbf{S}_{2}, \mathbf{Z}\right\rangle \\
& +\beta_{2}^{2}\left\|\mathbf{U}_{2}^{*}\right\|^{2}+2\left\langle\beta_{2} \mathbf{U}_{2}^{*}, \mathbf{Z}\right\rangle+\|\mathbf{Z}\|^{2} \\
& \\
\leq & 9\left(\alpha_{1}^{2}\left\|\mathbf{S}_{1}\right\|^{2}+\alpha_{2}^{2}\left\|\mathbf{S}_{2}\right\|^{2}+\beta_{1}^{2}\left\|\mathbf{U}_{1}^{*}\right\|^{2}+\beta_{2}^{2}\left\|\mathbf{U}_{2}^{*}\right\|^{2}+\|\mathbf{Z}\|^{2}\right) \\
\leq & 9\left(\alpha_{1}^{2}\left\|\mathbf{S}_{1}\right\|^{2}+\alpha_{2}^{2}\left\|\mathbf{S}_{2}\right\|^{2}+n P_{1}+n P_{2}+\|\mathbf{Z}\|^{2}\right) .
\end{aligned}
$$

where $a$ ) follows from upper bounding all inner products by (121). Thus, combining (172) with (171) gives

$$
\begin{aligned}
\left\|\hat{\mathbf{S}}_{1}\right\|^{2}-\left\|\hat{\mathbf{S}}_{1}^{\mathrm{G}}\right\|^{2} \leq & 4 n \sigma^{2}+16 \gamma_{13} n \sigma^{2}+36 n \gamma_{13}\left(P_{1}+P_{2}\right) \\
& +36 \gamma_{13}\left(\alpha_{1}^{2}\left\|\mathbf{S}_{1}\right\|^{2}+\alpha_{2}^{2}\left\|\mathbf{S}_{2}\right\|^{2}+\|\mathbf{Z}\|^{2}\right) .
\end{aligned}
$$

And combining (173) with (170) gives

$$
\begin{aligned}
\frac{1}{n} \mathrm{E}\left[\left\|\hat{\mathbf{S}}_{1}\right\|^{2}-\left\|\hat{\mathbf{S}}_{1}^{\mathrm{G}}\right\|^{2}\right] \leq & 4 \sigma^{2} \operatorname{Pr}\left[\mathcal{E}_{\hat{\mathbf{U}}}\right] \\
& +36 \gamma_{13}\left(P_{1}+P_{2}\right) \operatorname{Pr}\left[\mathcal{E}_{\hat{\mathbf{U}}}\right] \\
& +36 \gamma_{13}\left(\alpha_{1}^{2} \frac{1}{n} \mathrm{E}\left[\left\|\mathbf{S}_{1}\right\|^{2} \mid \mathcal{E}_{\hat{\mathbf{U}}}\right] \operatorname{Pr}\left[\mathcal{E}_{\hat{\mathbf{U}}}\right]\right. \\
& +\alpha_{2}^{2} \frac{1}{n} \mathrm{E}\left[\left\|\mathbf{S}_{2}\right\|^{2} \mid \mathcal{E}_{\hat{\mathbf{U}}}\right] \operatorname{Pr}\left[\mathcal{E}_{\hat{\mathbf{U}}}\right] \\
& \left.+\frac{1}{n} \mathrm{E}\left[\|\mathbf{Z}\|^{2} \mid \mathcal{E}_{\hat{\mathbf{U}}}\right] \operatorname{Pr}\left[\mathcal{E}_{\hat{\mathbf{U}}}\right]\right) .
\end{aligned}
$$

It now remains to upper bound the expectations on $\mathbf{S}_{1}, \mathbf{S}_{2}$ and $\mathbf{Z}$ on the RHS of (174). Since $\mathbf{S}_{1}, \mathbf{S}_{2}$ and $\mathbf{Z}$ are each Gaussian, their corresponding terms can be bounded in similar ways. We show here the derivation for $\mathbf{S}_{1}$.

$$
\begin{aligned}
\frac{1}{n} \mathrm{E}\left[\left\|\mathbf{S}_{1}\right\|^{2} \mid \mathcal{E}_{\hat{\mathbf{U}}}\right] \operatorname{Pr}\left[\mathcal{E}_{\hat{\mathbf{U}}}\right]= & \frac{1}{n} \mathrm{E}\left[\left\|\mathbf{S}_{1}\right\|^{2} \mid \mathcal{E}_{\hat{\mathbf{U}}} \cap \mathcal{E}_{\mathbf{S}}\right] \operatorname{Pr}\left[\mathcal{E}_{\hat{\mathbf{U}}} \cap \mathcal{E}_{\mathbf{S}}\right] \\
& +\frac{1}{n} \mathrm{E}\left[\left\|\mathbf{S}_{1}\right\|^{2} \mid \mathcal{E}_{\hat{\mathbf{U}}} \cap \mathcal{E}_{\mathbf{S}}^{c}\right] \operatorname{Pr}\left[\mathcal{E}_{\hat{\mathbf{U}}} \cap \mathcal{E}_{\mathbf{S}}^{c}\right] \\
\leq & \frac{1}{n} \mathrm{E}\left[\left\|\mathbf{S}_{1}\right\|^{2} \mid \mathcal{E}_{\mathbf{S}}\right] \operatorname{Pr}\left[\mathcal{E}_{\mathbf{S}}\right] \\
& +\sigma^{2}(1+\epsilon) \operatorname{Pr}\left[\mathcal{E}_{\hat{\mathbf{U}}}\right] \\
\leq & \sigma^{2}\left(\epsilon+\operatorname{Pr}\left[\mathcal{E}_{\mathbf{S}}\right]\right)+\sigma^{2}(1+\epsilon) \operatorname{Pr}\left[\mathcal{E}_{\hat{\mathbf{U}}}\right],
\end{aligned}
$$

where in the last step we have used Lemma E.10, For the expectations on $\mathbf{S}_{2}$ and $\mathbf{Z}$, we similarly obtain

$$
\frac{1}{n} \mathrm{E}\left[\left\|\mathbf{S}_{2}\right\|^{2} \mid \mathcal{E}_{\hat{\mathbf{U}}}\right] \operatorname{Pr}\left[\mathcal{E}_{\hat{\mathbf{U}}}\right] \leq \sigma^{2}\left(\epsilon+\operatorname{Pr}\left[\mathcal{E}_{\mathbf{S}}\right]\right)+\sigma^{2}(1+\epsilon) \operatorname{Pr}\left[\mathcal{E}_{\hat{\mathbf{U}}}\right],
$$


and

$$
\frac{1}{n} \mathrm{E}\left[\|\mathbf{Z}\|^{2} \mid \mathcal{E}_{\hat{\mathbf{U}}}\right] \operatorname{Pr}\left[\mathcal{E}_{\hat{\mathbf{U}}}\right] \leq N\left(\epsilon+\operatorname{Pr}\left[\mathcal{E}_{\mathbf{Z}}\right]\right)+N(1+\epsilon) \operatorname{Pr}\left[\mathcal{E}_{\hat{\mathbf{U}}}\right] .
$$

Thus, combining (175) - (177) with (174) gives

$$
\begin{aligned}
\frac{1}{n} \mathrm{E}\left[\left\|\hat{\mathbf{S}}_{1}\right\|^{2}-\left\|\hat{\mathbf{S}}_{1}^{\mathrm{G}}\right\|^{2}\right] \leq & 4 \sigma^{2} \operatorname{Pr}\left[\mathcal{E}_{\hat{\mathbf{U}}}\right]+16 \gamma_{13} \sigma^{2} \operatorname{Pr}\left[\mathcal{E}_{\hat{\mathbf{U}}}\right] \\
& +36 \gamma_{13}\left(P_{1}+P_{2}\right) \operatorname{Pr}\left[\mathcal{E}_{\hat{\mathbf{U}}}\right] \\
& +36 \gamma_{13}\left(\left(P_{1}+P_{2}\right)\left(\epsilon+\operatorname{Pr}\left[\mathcal{E}_{\mathbf{S}}\right]\right)\right. \\
& +\left(P_{1}+P_{2}+N\right)(1+\epsilon) \operatorname{Pr}\left[\mathcal{E}_{\hat{\mathbf{U}}}\right] \\
& \left.+N\left(\epsilon+\operatorname{Pr}\left[\mathcal{E}_{\mathbf{Z}}\right]\right)\right) \\
\leq & 4\left(\sigma^{2}\left(1+4 \gamma_{13}\right)+9 \gamma_{13}\left(P_{1}+P_{2}+N\right)(1+\epsilon)\right) \operatorname{Pr}\left[\mathcal{E}_{\hat{\mathbf{U}}}\right] \\
& +36 \gamma_{13}\left(\left(P_{1}+P_{2}\right) \operatorname{Pr}\left[\mathcal{E}_{\mathbf{S}}\right]+N \operatorname{Pr}\left[\mathcal{E}_{\mathbf{Z}}\right]+\left(P_{1}+P_{2}+N\right) \epsilon\right) .
\end{aligned}
$$

Based on Lemma G.3 and Lemma G.4, the proof of Proposition G.1 now follows easily.

Proof of Proposition G.1.

$$
\begin{aligned}
\frac{1}{n} \mathrm{E}\left[\left\|\mathbf{S}_{1}-\hat{\mathbf{S}}_{1}\right\|^{2}\right]-\frac{1}{n} \mathrm{E}\left[\left\|\mathbf{S}_{1}-\hat{\mathbf{S}}_{1}^{\mathrm{G}}\right\|^{2}\right] & =\frac{1}{n} \mathrm{E}\left[\left\|\mathbf{S}_{1}-\hat{\mathbf{S}}_{1}\right\|^{2}-\left\|\mathbf{S}_{1}-\hat{\mathbf{S}}_{1}^{\mathrm{G}}\right\|^{2}\right] \\
= & \frac{1}{n}\left(\mathrm{E}\left[\left\|\mathbf{S}_{1}\right\|^{2}\right]-2 \mathrm{E}\left[\left\langle\mathbf{S}_{1}, \hat{\mathbf{S}}_{1}\right\rangle\right]+\mathrm{E}\left[\left\|\hat{\mathbf{S}}_{1}\right\|^{2}\right]\right. \\
& \left.\quad-\mathrm{E}\left[\left\|\mathbf{S}_{1}\right\|^{2}\right]+2 \mathrm{E}\left[\left\langle\mathbf{S}_{1}, \hat{\mathbf{S}}_{1}^{\mathrm{G}}\right\rangle\right]-\mathrm{E}\left[\left\|\hat{\mathbf{S}}_{1}^{\mathrm{G}}\right\|^{2}\right]\right) \\
= & 2 \frac{1}{n} \mathrm{E}\left[\left\langle\mathbf{S}_{1}, \hat{\mathbf{S}}_{1}^{\mathrm{G}}-\hat{\mathbf{S}}_{1}\right\rangle\right]+\frac{1}{n} \mathrm{E}\left[\left\|\hat{\mathbf{S}}_{1}\right\|^{2}-\left\|\hat{\mathbf{S}}_{1}^{\mathrm{G}}\right\|^{2}\right] \\
\leq & 2 \sigma^{2}\left(\epsilon+17 \operatorname{Pr}\left[\mathcal{E}_{\mathbf{S}}\right]+(17+\epsilon) \operatorname{Pr}\left[\mathcal{E}_{\hat{\mathbf{U}}}\right]\right) \\
& +4\left(\sigma^{2}\left(1+4 \gamma_{13}\right)+9 \gamma_{13}\left(P_{1}+P_{2}+N\right)(1+\epsilon)\right) \operatorname{Pr}\left[\mathcal{E}_{\hat{\mathbf{U}}}\right] \\
& +36 \gamma_{13}\left(\left(P_{1}+P_{2}\right) \operatorname{Pr}\left[\mathcal{E}_{\mathbf{S}}\right]+N \operatorname{Pr}\left[\mathcal{E}_{\mathbf{Z}}\right]+\left(P_{1}+P_{2}+N\right) \epsilon\right) \\
= & \xi_{1} \operatorname{Pr}\left[\mathcal{E}_{\hat{\mathbf{U}}}\right]+\xi_{2} \operatorname{Pr}\left[\mathcal{E}_{\mathbf{S}}\right]+\xi_{3} \operatorname{Pr}\left[\mathcal{E}_{\mathbf{Z}}\right]+\xi_{4} \epsilon
\end{aligned}
$$

where in step $a$ ) we have used LemmaG.3 and LemmaG.4 and where $\xi_{\ell}, \ell \in\{1,2,3,4\}$, depend only on $\sigma^{2}, \gamma_{13}, P_{1}, P_{2}$ and $N$. Combining (178) with Lemma E.1, Lemma E.2 and Lemma E.3 gives that for every $\delta>0$ and $0.3>\epsilon>0$, there exists an $n^{\prime}(\delta, \epsilon)>0$ such that for all $\left(R_{1}, R_{2}\right) \in \mathcal{R}^{\prime}(\epsilon)$ and $n>n^{\prime}(\delta, \epsilon)$

$$
\frac{1}{n} \mathrm{E}\left[\left\|\mathbf{S}_{1}-\hat{\mathbf{S}}_{1}\right\|^{2}\right]-\frac{1}{n} \mathrm{E}\left[\left\|\mathbf{S}_{1}-\hat{\mathbf{S}}_{1}^{\mathrm{G}}\right\|^{2}\right]<\xi_{1}^{\prime} \delta+\xi_{4} 2^{\prime} \epsilon,
$$

where $\xi_{1}^{\prime}$ and $\xi_{2}^{\prime}$ depend only on $\sigma^{2}, \gamma_{13}, P_{1}, P_{2}$ and $N$. 


\section{G.3 Upper Bound on Expected Distortion}

We now derive an upper bound on the achievable distortion for the proposed vectorquantizer scheme. By Corollary G.1, it suffices to analyze the genie-aided scheme. Using that $\hat{\mathbf{S}}_{1}^{\mathrm{G}}=\gamma_{11} \mathbf{U}_{1}^{*}+\gamma_{12} \mathbf{U}_{2}^{*}+\gamma_{13} \mathbf{Y}$, we have

$$
\begin{aligned}
\frac{1}{n} \mathrm{E}\left[\left\|\mathbf{S}_{1}-\hat{\mathbf{S}}_{1}^{\mathrm{G}}\right\|^{2}\right]=\frac{1}{n}( & \mathrm{E}\left[\left\|\mathbf{S}_{1}\right\|^{2}\right]-2 \gamma_{11} \mathrm{E}\left[\left\langle\mathbf{S}_{1}, \mathbf{U}_{1}^{*}\right\rangle\right]-2 \gamma_{12} \mathrm{E}\left[\left\langle\mathbf{S}_{1}, \mathbf{U}_{2}^{*}\right\rangle\right] \\
& -2 \gamma_{13} \mathrm{E}\left[\left\langle\mathbf{S}_{1}, \mathbf{Y}\right\rangle\right]+\gamma_{11}^{2} \mathrm{E}\left[\left\|\mathbf{U}_{1}^{*}\right\|^{2}\right]+2 \gamma_{11} \gamma_{12} \mathrm{E}\left[\left\langle\mathbf{U}_{1}^{*}, \mathbf{U}_{2}^{*}\right\rangle\right] \\
& +2 \gamma_{11} \gamma_{13} \mathrm{E}\left[\left\langle\mathbf{U}_{1}^{*}, \mathbf{Y}\right\rangle\right]+\gamma_{12}^{2} \mathrm{E}\left[\left\|\mathbf{U}_{2}^{*}\right\|^{2}\right] \\
& \left.+2 \gamma_{12} \gamma_{13} \mathrm{E}\left[\left\langle\mathbf{U}_{2}^{*}, \mathbf{Y}\right\rangle\right]+\gamma_{13}^{2} \mathrm{E}\left[\|\mathbf{Y}\|^{2}\right]\right)
\end{aligned}
$$

Some of the expectation terms are bounded straightforwardly. In particular, we have $\mathrm{E}\left[\left\|\mathbf{S}_{1}\right\|^{2}\right]=n \sigma^{2}, \mathrm{E}\left[\left\|\mathbf{U}_{1}^{*}\right\|^{2}\right]=n \sigma^{2}\left(1-2^{-2 R_{1}}\right)$, and $\mathrm{E}\left[\left\|\mathbf{U}_{2}^{*}\right\|^{2}\right]=n \sigma^{2}\left(1-2^{-2 R_{2}}\right)$. For three further terms we take over the bounds from the analysis of the vector-quantizer scheme. That is, by Lemma E.14 we have that for every $\delta>0$ and $0<\epsilon<0.3$ and every positive integer $n$

$$
\begin{aligned}
\frac{1}{n} \mathrm{E}\left[\left\langle\mathbf{S}_{1}, \mathbf{U}_{1}^{*}\right\rangle\right] & \geq \sigma^{2}\left(1-2^{-2 R_{1}}\right)-\zeta_{1}(\delta, \epsilon) \\
& =\mathrm{c}_{11}-\zeta_{1}(\delta, \epsilon),
\end{aligned}
$$

where $\zeta_{1}(\delta, \epsilon)$ is such that $\lim _{\delta, \epsilon \rightarrow 0} \zeta_{1}(\delta, \epsilon)=0$. By Lemma E.15 we have that for every $\delta>0$ and $0<\epsilon<0.3$ there exists an $n_{2}^{\prime}(\delta, \epsilon) \in \mathbb{N}$ such that for all $n>n_{2}^{\prime}(\delta, \epsilon)$

$$
\begin{aligned}
\frac{1}{n} \mathrm{E}\left[\left\langle\mathbf{U}_{1}^{*}, \mathbf{U}_{2}^{*}\right\rangle\right] & \leq \sigma^{2} \rho\left(1-2^{-2 R_{1}}\right)\left(1-2^{-2 R_{2}}\right)+\zeta_{2}(\delta, \epsilon) \\
& =\mathrm{k}_{12}+\zeta_{2}(\delta, \epsilon),
\end{aligned}
$$

where $\zeta_{2}(\delta, \epsilon)$ is such that $\lim _{\delta, \epsilon \rightarrow 0} \zeta_{2}(\delta, \epsilon)=0$. And by Lemma E.16 we have that for every $\delta>0$ and $0<\epsilon<0.3$ there exists an $n^{\prime}(\delta, \epsilon) \in \mathbb{N}$ such that for all $n>n^{\prime}(\delta, \epsilon)$

$$
\begin{aligned}
\frac{1}{n} \mathrm{E}\left[\left\langle\mathbf{S}_{1}, \mathbf{U}_{2}^{*}\right\rangle\right] & \geq \sigma^{2} \rho\left(1-2^{-2 R_{2}}\right)-\zeta_{3}(\delta, \epsilon) \\
& =\mathrm{c}_{12}-\zeta_{3}(\delta, \epsilon),
\end{aligned}
$$

where $\zeta_{3}(\delta, \epsilon)$ is such that $\lim _{\delta, \epsilon \rightarrow 0} \zeta_{3}(\delta, \epsilon)=0$. Next, recalling that $\mathbf{Y}=\alpha_{1} \mathbf{S}_{1}+\beta_{1} \mathbf{U}_{1}^{*}+$ $\alpha_{2} \mathbf{S}_{2}+\beta_{2} \mathbf{U}_{2}^{*}+\mathbf{Z}$, gives

$$
\begin{aligned}
\frac{1}{n} \mathrm{E}\left[\left\langle\mathbf{S}_{1}, \mathbf{Y}\right\rangle\right]= & \frac{1}{n}\left(\alpha_{1} \mathrm{E}\left[\left\|\mathbf{S}_{1}\right\|^{2}\right]+\beta_{1} \mathrm{E}\left[\left\langle\mathbf{S}_{1}, \mathbf{U}_{1}^{*}\right\rangle\right]+\alpha_{2} \mathrm{E}\left[\left\langle\mathbf{S}_{1}, \mathbf{S}_{2}\right\rangle\right]\right. \\
& \quad+\beta_{2} \mathrm{E}\left[\left\langle\mathbf{S}_{1}, \mathbf{U}_{2}^{*}\right\rangle\right]+\underbrace{\mathrm{E}\left[\left\langle\mathbf{S}_{1}, \mathbf{Z}\right\rangle\right]}_{=0}) \\
& \stackrel{a)}{\geq} \alpha_{1} \sigma^{2}+\beta_{1}\left(c_{11}-\zeta_{1}(\delta, \epsilon)\right)+\alpha_{2} \rho \sigma^{2}+\beta_{2}\left(c_{12}-\zeta_{3}(\delta, \epsilon)\right) \\
= & c_{13}-\zeta_{4}(\delta, \epsilon),
\end{aligned}
$$

where in $a$ ) we have used (180), (181) and (182), and where $\zeta_{4}(\delta, \epsilon)$ is such that $\lim _{\delta, \epsilon \rightarrow 0} \zeta_{4}(\delta, \epsilon)=0$. For the remaining terms in (179), it can be shown, similarly 
as for (180) and (182), that for every $\delta>0$ and $0<\epsilon<0.3$ there exists an $n^{\prime \prime}(\delta, \epsilon) \in \mathbb{N}$ such that for all $n>n^{\prime \prime}(\delta, \epsilon)$

$$
\begin{aligned}
& \frac{1}{n} \mathrm{E}\left[\left\langle\mathbf{S}_{1}, \mathbf{U}_{1}^{*}\right\rangle\right] \leq \mathrm{c}_{11}+\zeta_{5}(\delta, \epsilon) \\
& \frac{1}{n} \mathrm{E}\left[\left\langle\mathbf{S}_{2}, \mathbf{U}_{1}^{*}\right\rangle\right] \leq \mathrm{c}_{21}+\zeta_{6}(\delta, \epsilon) \\
& \frac{1}{n} \mathrm{E}\left[\left\langle\mathbf{S}_{1}, \mathbf{U}_{2}^{*}\right\rangle\right] \leq \mathrm{c}_{12}+\zeta_{7}(\delta, \epsilon) \\
& \frac{1}{n} \mathrm{E}\left[\left\langle\mathbf{S}_{2}, \mathbf{U}_{2}^{*}\right\rangle\right] \leq \mathrm{c}_{22}+\zeta_{8}(\delta, \epsilon),
\end{aligned}
$$

where $\zeta_{j}(\delta, \epsilon), j \in\{5, \ldots 8\}$, are such that $\lim _{\delta, \epsilon \rightarrow 0} \zeta_{j}(\delta, \epsilon)=0$. Using (181) and (184) - (187), we now get that for every $\delta>0$ and $0<\epsilon<0.3$ there exists an $\tilde{n}_{1}(\delta, \epsilon) \in \mathbb{N}$ such that for all $n>\tilde{n}_{1}(\delta, \epsilon)$

$$
\begin{aligned}
\frac{1}{n} \mathrm{E}\left[\left\langle\mathbf{U}_{1}^{*}, \mathbf{Y}\right\rangle\right]=\frac{1}{n}( & \alpha_{1} \mathrm{E}\left[\left\langle\mathbf{U}_{1}^{*}, \mathbf{S}_{1}\right\rangle\right]+\beta_{1} \mathrm{E}\left[\left\|\mathbf{U}_{1}^{*}\right\|^{2}\right]+\alpha_{2} \mathrm{E}\left[\left\langle\mathbf{U}_{1}^{*}, \mathbf{S}_{2}\right\rangle\right] \\
& +\beta_{2} \mathrm{E}\left[\left\langle\mathbf{U}_{1}^{*}, \mathbf{U}_{2}^{*}\right\rangle\right]+\underbrace{\mathrm{E}\left[\left\langle\mathbf{U}_{1}^{*}, \mathbf{Z}\right\rangle\right]}_{=0}) \\
\leq & \alpha_{1}\left(\mathrm{k}_{11}+\zeta_{5}(\delta, \epsilon)\right)+\beta_{1} \mathrm{k}_{11}+\alpha_{2}\left(\mathrm{c}_{21}+\zeta_{6}(\delta, \epsilon)\right) \\
& +\beta_{2}\left(\mathrm{k}_{12}+\zeta_{2}(\delta, \epsilon)\right) \\
= & \mathrm{k}_{13}+\tilde{\zeta}_{1}(\delta, \epsilon),
\end{aligned}
$$

where $\tilde{\zeta}_{1}(\delta, \epsilon)$ is such that $\lim _{\delta, \epsilon \rightarrow 0} \tilde{\zeta}_{1}(\delta, \epsilon)=0$. Similarly, it can be shown that for every $\delta>0$ and $0<\epsilon<0.3$ there exists an $\tilde{n}_{2}(\delta, \epsilon) \in \mathbb{N}$ such that for all $n>\tilde{n}_{2}(\delta, \epsilon)$

$$
\frac{1}{n} \mathrm{E}\left[\left\langle\mathbf{U}_{2}^{*}, \mathbf{Y}\right\rangle\right] \leq \mathrm{k}_{23}+\tilde{\zeta}_{2}(\delta, \epsilon),
$$

where $\tilde{\zeta}_{2}(\delta, \epsilon)$ is such that $\lim _{\delta, \epsilon \rightarrow 0} \tilde{\zeta}_{2}(\delta, \epsilon)=0$. And finally, we have that for every $\delta>0$ and $0<\epsilon<0.3$ there exists an $\tilde{n}_{3}(\delta, \epsilon) \in \mathbb{N}$ such that for all $n>\tilde{n}_{3}(\delta, \epsilon)$

$$
\begin{aligned}
\frac{1}{n} \mathrm{E}\left[\|\mathbf{Y}\|^{2}\right]=\frac{1}{n} & \alpha_{1}^{2} \mathrm{E}\left[\left\|\mathbf{S}_{1}\right\|^{2}\right]+2 \alpha_{1} \beta_{1} \mathrm{E}\left[\left\langle\mathbf{S}_{1}, \mathbf{U}_{1}^{*}\right\rangle\right]+2 \alpha_{1} \alpha_{2} \mathrm{E}\left[\left\langle\mathbf{S}_{1}, \mathbf{S}_{2}\right\rangle\right]+2 \alpha_{1} \beta_{2} \mathrm{E}\left[\left\langle\mathbf{S}_{1}, \mathbf{U}_{2}^{*}\right\rangle\right] \\
& +2 \alpha_{1} \underbrace{\mathrm{E}\left[\left\langle\mathbf{S}_{1}, \mathbf{Z}\right\rangle\right]}_{=0}+\beta_{1}^{2} \mathrm{E}\left[\left\|\mathbf{U}_{1}^{*}\right\|^{2}\right]+2 \beta_{1} \alpha_{2} \mathrm{E}\left[\left\langle\mathbf{U}_{1}^{*}, \mathbf{S}_{2}\right\rangle\right]+2 \beta_{1} \beta_{2} \mathrm{E}\left[\left\langle\mathbf{U}_{1}^{*}, \mathbf{U}_{2}^{*}\right\rangle\right] \\
& +2 \beta_{1} \underbrace{\mathrm{E}\left[\left\langle\mathbf{U}_{1}^{*}, \mathbf{Z}\right\rangle\right]}_{=0}+\alpha_{2}^{2} \mathrm{E}\left[\left\|\mathbf{S}_{2}\right\|^{2}\right]+2 \alpha_{2} \beta_{2} \mathrm{E}\left[\left\langle\mathbf{S}_{2}, \mathbf{U}_{2}^{*}\right\rangle\right]+2 \alpha_{2} \underbrace{\mathrm{E}\left[\left\langle\mathbf{S}_{2}, \mathbf{Z}\right\rangle\right]}_{=0} \\
& +\beta_{2}^{2} \mathrm{E}\left[\left\|\mathbf{U}_{2}^{*}\right\|^{2}\right]+2 \beta_{2} \underbrace{\mathrm{E}\left[\left\langle\mathbf{U}_{2}^{*}, \mathbf{Z}\right\rangle\right]}_{=0}+\mathrm{E}\left[\|\mathbf{Z}\|^{2}\right]) \\
\leq & \alpha_{1}^{2} \sigma^{2}+2 \alpha_{1} \beta_{1}\left(\mathrm{c}_{11}+\zeta_{5}(\delta, \epsilon)\right)+2 \alpha_{1} \alpha_{2} \rho \sigma^{2}+2 \alpha_{1} \beta_{2}\left(\mathrm{c}_{12}+\zeta_{7}(\delta, \epsilon)\right) \\
& +\beta_{1}^{2} \mathrm{k}_{11}+2 \beta_{1} \alpha_{2}\left(\mathrm{c}_{21}+\zeta_{6}(\delta, \epsilon)\right)+2 \beta_{1} \beta_{2}\left(\mathrm{k}_{12}+\zeta_{2}(\delta, \epsilon)\right) \\
& +\alpha_{2}^{2} \sigma^{2}+2 \alpha_{2} \beta_{2}\left(\mathrm{c}_{22}+\zeta_{8}(\delta, \epsilon)\right)
\end{aligned}
$$




$$
\begin{gathered}
+\beta_{2}^{2} \mathrm{k}_{22}+N \\
\left.=\mathrm{k}_{33}+\tilde{\zeta}_{3}(\delta, \epsilon)\right),
\end{gathered}
$$

where $\tilde{\zeta}_{3}(\delta, \epsilon)$ is such that $\lim _{\delta, \epsilon \rightarrow 0} \tilde{\zeta}_{3}(\delta, \epsilon)=0$. Thus, combining (180) - (183) and (188) - (190) with (179) gives that for every $\delta>0$ and $0<\epsilon<0.3$ there exists an $n^{\prime}(\delta, \epsilon) \in \mathbb{N}$ such that for all $n>n^{\prime}(\delta, \epsilon)$

$$
\begin{aligned}
& \frac{1}{n} \mathrm{E}\left[\left\|\mathbf{S}_{1}-\hat{\mathbf{S}}_{1}^{\mathrm{G}}\right\|^{2}\right] \leq \sigma^{2}-2 \gamma_{11} \mathrm{c}_{11}-2 \gamma_{12} \mathrm{c}_{12}-2 \gamma_{13} \mathrm{c}_{13}+\gamma_{11}^{2} \mathrm{k}_{11}+2 \gamma_{11} \gamma_{12} \mathrm{k}_{12} \\
& +2 \gamma_{11} \gamma_{13} \mathrm{k}_{13}+\gamma_{12}^{2} \mathrm{k}_{22}+\gamma_{12} \gamma_{13} \mathrm{k}_{23}+\gamma_{13}^{2} \mathrm{k}_{33}+\zeta^{\prime}(\delta, \epsilon) \\
& =\sigma^{2}-2 \gamma_{11} c_{11}-2 \gamma_{12} c_{12}-2 \gamma_{13} c_{13} \\
& +\left(\begin{array}{lll}
\gamma_{11} & \gamma_{12} & \gamma_{13}
\end{array}\right) \mathrm{K}\left(\begin{array}{l}
\gamma_{11} \\
\gamma_{12} \\
\gamma_{13}
\end{array}\right)+\zeta^{\prime}(\delta, \epsilon) \\
& \stackrel{a)}{=} \sigma^{2}-2 \gamma_{11} \mathrm{c}_{11}-2 \gamma_{12} \mathrm{c}_{12}-2 \gamma_{13} \mathrm{c}_{13} \\
& +\left(\begin{array}{lll}
\gamma_{11} & \gamma_{12} & \gamma_{13}
\end{array}\right) \mathrm{KK}^{-1}\left(\begin{array}{l}
\mathrm{c}_{11} \\
\mathrm{c}_{12} \\
\mathrm{c}_{13}
\end{array}\right)+\zeta^{\prime}(\delta, \epsilon) \\
& =\sigma^{2}-2 \gamma_{11} \mathrm{c}_{11}-2 \gamma_{12} \mathrm{c}_{12}-2 \gamma_{13} \mathrm{c}_{13} \\
& +\left(\begin{array}{lll}
\gamma_{11} & \gamma_{12} & \gamma_{13}
\end{array}\right)\left(\begin{array}{l}
\mathrm{c}_{11} \\
\mathrm{c}_{12} \\
\mathrm{c}_{13}
\end{array}\right)+\zeta^{\prime}(\delta, \epsilon) \\
& =\sigma^{2}-\gamma_{11} \mathrm{c}_{11}-\gamma_{12} \mathrm{c}_{12}-\gamma_{13} \mathrm{c}_{13}+\zeta^{\prime}(\delta, \epsilon),
\end{aligned}
$$

where we have used the shorthand notation $\mathrm{K}$ for $\mathrm{K}\left(R_{1}, R_{2}\right)$, and where in $a$ ) we have used the definition of the coefficients $\gamma_{i j}$ in (33), and where $\zeta^{\prime}(\delta, \epsilon)$ is such that $\lim _{\delta, \epsilon \rightarrow 0} \zeta^{\prime}(\delta, \epsilon)=0$. Now, letting in (191) first $n \rightarrow \infty$ and then $\delta, \epsilon \rightarrow 0$, and combining the result with Corollary G.1 gives

$$
\varlimsup_{n \rightarrow \infty} \mathrm{E}\left[\left\|\mathbf{S}_{1}-\hat{\mathbf{S}}_{1}\right\|^{2}\right] \leq \sigma^{2}-\gamma_{11} \mathrm{c}_{11}-\gamma_{12} \mathrm{c}_{12}-\gamma_{13} \mathrm{c}_{13},
$$

whenever $\left(R_{1}, R_{2}\right)$ satisfy

$$
\begin{aligned}
R_{1} & <\frac{1}{2} \log _{2}\left(\frac{\beta_{1}^{\prime 2}\left\|\mathbf{U}_{1}\right\|^{2}\left(1-\tilde{\rho}^{2}\right)+N^{\prime}}{N^{\prime}\left(1-\tilde{\rho}^{2}\right)}\right) \\
R_{2} & <\frac{1}{2} \log _{2}\left(\frac{\beta_{2}^{\prime 2}\left\|\mathbf{U}_{2}\right\|^{2}\left(1-\tilde{\rho}^{2}\right)+N^{\prime}}{N^{\prime}\left(1-\tilde{\rho}^{2}\right)}\right) \\
R_{1}+R_{2} & <\frac{1}{2} \log _{2}\left(\frac{\beta_{1}^{\prime 2}\left\|\mathbf{U}_{1}\right\|^{2}+\beta_{2}^{\prime 2}\left\|\mathbf{U}_{2}\right\|^{2}+2 \tilde{\rho} \beta_{1}^{\prime} \beta_{2}^{\prime}\left\|\mathbf{U}_{1}\right\|\left\|\mathbf{U}_{2}\right\|+N^{\prime}}{N^{\prime}\left(1-\tilde{\rho}^{2}\right)}\right) .
\end{aligned}
$$




\section{References}

[1] T. J. Goblick, "Theoretical Limitations on the Transmission of Data from Analog Sources", IEEE Transaction on Information Theory, IT-11(4): pp. 558-567, October 1965.

[2] T. M. Cover, A. El-Gamal and M. Salehi, "Multiple Access Channels with Arbitrarily Correlated Sources", IEEE Transactions on Information Theory, IT-26(6): pp. 648-657, November 1980.

[3] G. Dueck, "A Note on the Multiple Access Channel with Correlated Sources", IEEE Transactions on Information Theory, IT-27(2): pp. 232-235, March 1981.

[4] W. Kang and S. Ulukus, "A Single-letter Upper Bound for the Sum Rate of Multiple Access Channels with Correlated Sources", November 2005, http://arxiv.org/pdf/cs.IT/0511096.

[5] Y. Oohama, "Gaussian Multiterminal Source Coding", IEEE Transactions on Information Theory, IT-43(6), pp. 1912-1923, November 1997.

[6] A. B. Wagner, S. Tavildar and P. Viswanath, "Rate Region of the Quadratic Gaussian Two-Encoder Source-Coding Problem", IEEE Transactions on Information Theory, IT-54(5), pp. 1938-1961, May, 2008.

[7] H. Vishvanathan and T. Berger, "The Quadratic Gaussian CEO Problem", IEEE Transactions on Information Theory, IT-43, pp. 1549-1559, September 1997.

[8] Y. Oohama, "The Rate-Distortion Function for the Quadratic Gaussian CEO Problem", IEEE Transactions on Information Theory, IT-44(3), pp. 1057-1070, May 1998.

[9] M. Salehi, "Multiple-Access Channels with Correlated Sources", in Proceedings IEEE International Symposium on Information Theory (ISIT), p. 198, September 17-22, 1995.

[10] A. D. Murugan, P. K. Gopala, H. El Gamal, "Correlated Source over Wireless Channels: Cooperative Source-Channel Coding", IEEE Journal on Selected Areas in Communications, 22(6), pp. 988-998, August 2004.

[11] M. Gastpar, "Uncoded transmission is exactly optimal for a simple Gaussian sensor network", in Proceedings Information Theory and Applications Workshop, San Diego, CA, USA, January 29 - February 2, 2007.

[12] A. Lapidoth and S. Tinguely, "Sending a Bi-Variate Gaussian Source over a Gaussian MAC with Feedback", in preparation.

[13] A. Lapidoth and S. Tinguely, "Sending a Bi-Variate Gaussian Source over a Gaussian MAC with Feedback", in Proceedings IEEE International Symposium on Information Theory, June 24-29, 2007. 
[14] J. Xiao and Z. Luo, "Compression of Correlated Gaussian Sources under Individual Distortion Criteria", in Proceedings 43rd Allerton Conference, Illinois, September, 2005 .

[15] Yu. A. Rozanov (translated by A. Feinstein), Stationary Random Processes, Holden-Day, 1967.

[16] R. Ahlswede, "Multi-Way Communication Channels", in 2nd International Symposium on Information Theory, (Tsahkadsor, Armenian S. S. R.), pp. 23-52, Hungarian Academy of Sciences, 1971.

[17] H. Liao, Multiple Access Channels, Ph.D. thesis, Dept. of Electrical Engineering, University of Hawaii, Honolulu, 1972.

[18] T. M. Cover, J. A. Thomas, "Elements of Information Theory", John Wiley \& Sons, New York, 1991.

[19] S. Bross, A. Lapidoth, S. Tinguely, "Superimposed Coded and Uncoded Transmissions of a Gaussian Source over the Gaussian Channel", in Proceedings IEEE International Symposium on Information Theory, July 9 - July 14, 2006.

[20] A. A. El Gamal and T. M. Cover, "Achievable Rates for Multiple Descriptions", IEEE Transactions on Information Theory, vol. IT-28(6), November 1982.

[21] H. S. Witsenhausen, "On Sequences of Pairs of Dependent Random Variables", SIAM Journal on Applied Mathematics, vol. 28(1), pp. 100-113, January 1975.

[22] R. A. Horn and C. R. Johnson, Matrix Analysis, Cambridge University Press, 1985 .

[23] C. E. Shannon, "Probability of Error for Optimal Codes in a Gaussian Channel", Bell System Technical Journal, vol. 38, pp. 611-656, May 1959.

[24] R. L. Graham, D. E. Knuth, and O. Patashnik, Concrete Mathematics: A Foundation for Computer Science, 2nd ed. Reading, MA: Addison-Wesley, 1994.

[25] A. D. Wyner, "Random Packings and Coverings of the Unit n-Sphere", Bell System Technical Journal, vol. 46, pp. 2111-2118, November 1967. 